\title{
Radiation planning for image guided preclinical radiotherapy
}

Citation for published version (APA):

van Hoof, S. J. (2019). Radiation planning for image guided preclinical radiotherapy. [Doctoral Thesis, Maastricht University]. Maastricht University. https://doi.org/10.26481/dis.20190703sh

Document status and date:

Published: 01/01/2019

DOI:

10.26481/dis.20190703sh

Document Version:

Publisher's PDF, also known as Version of record

\section{Please check the document version of this publication:}

- A submitted manuscript is the version of the article upon submission and before peer-review. There can be important differences between the submitted version and the official published version of record.

People interested in the research are advised to contact the author for the final version of the publication, or visit the DOI to the publisher's website.

- The final author version and the galley proof are versions of the publication after peer review.

- The final published version features the final layout of the paper including the volume, issue and page numbers.

Link to publication

\footnotetext{
General rights rights.

- You may freely distribute the URL identifying the publication in the public portal. please follow below link for the End User Agreement:

www.umlib.nl/taverne-license

Take down policy

If you believe that this document breaches copyright please contact us at:

repository@maastrichtuniversity.nl

providing details and we will investigate your claim.
}

Copyright and moral rights for the publications made accessible in the public portal are retained by the authors and/or other copyright owners and it is a condition of accessing publications that users recognise and abide by the legal requirements associated with these

- Users may download and print one copy of any publication from the public portal for the purpose of private study or research.

- You may not further distribute the material or use it for any profit-making activity or commercial gain

If the publication is distributed under the terms of Article $25 \mathrm{fa}$ of the Dutch Copyright Act, indicated by the "Taverne" license above, 
doctoral thesis

\section{RADIATION PLANNING FOR IMAGE GUIDED PRECLINICAL RADIOTHERAPY}

Stefan van Hoof

2019 
(c) Stefan van Hoof, Maastricht 2019.

No part of this publication may be reproduced in any form without prior written permission of the holder of the copyrights.

Cover The cover represents an artistic representation of the multi-layer modeling and progressive simplification of the real world, to be used for predictions and analysis. In particular for this dissertation, irradiation treatment modelling for which geometries are progressively simplified until they fit the available computing and time resources. A surface render of a part of the spine and ribs of a mouse is shown, created using a high resolution computed tomography image from the preclinical radiotherapy research platform used for the work presented in this dissertation. The cross section of the mouse spine is about $3 \mathrm{~mm}$.

Cover design Stefan van Hoof

Printed by Gildeprint - the Netherlands

ISBN $\quad 9789463237123$ 


\title{
RADIATION PLANNING FOR IMAGE GUIDED PRECLINICAL RADIOTHERAPY
}

\author{
DISSERTATION \\ To obtain the degree of Doctor at Maastricht University, \\ on the authority of the Rector Magnificus, \\ prof. dr. Rianne M. Letschert, \\ in accordance with the decision of the Board of Deans, \\ to be defended in public \\ on Wednesday the $3^{\text {rd }}$ of July 2019 , at $16: 00$ hours \\ by
}

Stefan Josephus van Hoof 


\section{Supervisor}

prof. dr. ir. F. J. W. Verhaegen

\section{Assessment Committee}

prof. dr. D. K. M. De Ruysscher (chair)

dr. L.J. Dubois

prof. dr. D. Georg (Medical University of Vienna, Austria)

prof. dr. C. Vanhove (Ghent University, Belgium) 


\section{Contents}

1 Introduction 1

2 A review of treatment planning for precision image guided photon beam preclinical animal radiation studies

3 Development and validation of a treatment planning system for small animal radiotherapy: SmART-Plan

4 A framework for inverse planning of beam-on times for 3D small animal radiotherapy using interactive multi-objective optimisation

5 Dose painting by dynamic irradiation delivery on an image guided small animal radiotherapy platform

6 An image guided Small Animal Radiation Therapy Platform (SmART) to monitor glioblastoma progression and therapy response

7 General discussion

Summary

Samenvatting

Valorisation

Acknowledgments

Curriculum Vitae 

1

Introduction 



\subsection{Clinical radiotherapy}

Genetic damage accumulates during our lifetime as our body is unable to repair all genomic damage and mutant alleles are formed. Resulting abnormalities that lead to uncontrolled cell division and can spread into other tissues, are categorized as cancer. About $30 \%$ of the people have cancer by the end of their lifespan [1]. Cancer has risen to the leading cause of death in the Netherlands [2], and is expected to become a major cause of mortality in all regions of the world [3, 4]. Cancer causes immense unquantifiable human suffering and huge economic burden [5].

The principal basis for cancer treatment is simple but extremely difficult to apply in practice. All affected cells need to be removed, controlled or neutralized. One expedient is the use of ionization radiation which, in fundamental contrast to non-ionization radiation such as sunlight, carries enough energy to break covalent bonds between atoms and electrons. In particular, this can lead to double strand breaks in DNA molecules that can induce cell death, and can therefore be used to destroy tumour tissue [6]. Radiotherapy is the use of ionizing radiation as a means for treatment, against cancer or other disorders.

Radiotherapy is an effective, flexible and minimally invasive treatment modality that is used as primary, adjuvant, neoadjuvant or palliative treatment. Modern radiotherapy is a synergy of medicine, physics, biology, engineering and computer science, and the result of more than a century of evolution. The use of photon beams to neutralize cancers cells inherently causes damage to normal tissues. Therefore, radiotherapy treatments remain a balance between tumour control probability and normal tissue toxicity. The optimal trade-off for a treatment is difficult to define and plan. Therefore, radiation delivery is carefully planned using sophisticated algorithms collectively known as a treatment planning system (TPS) to maximise therapeutic ratio.

External beam radiotherapy (EBRT) using a linear accelerator (linac) is the most common method for clinical irradiations. Medical engineers have developed linacs into machines that can deliver highly modulated conformal radiation beams from nearly any incident direction. By combining beams with varying shapes and intensity distributions from different directions, the radiation dose in the tumour is increased while minimizing the dose to normal tissue. The use of a small number of static step-andshoot beam directions in intensity modulated radiotherapy (IMRT) [7] has evolved into dynamic radiation delivery with volumetric arc therapy (VMAT) [8] treatments. Multileaf collimators (MLCs) create complex superpositions of larger and smaller beams 
with varying dose rates, and daily image guidance can be achieved using e.g. online kilovolt cone beam CT imaging. Medical equipment companies are developing technologies to improve the integration of different imaging and treatment modalities into the same room or even into the same device. Innovative equipment such as the PET-CT, PET-MR and more recently the MR-linac, enable multi-parametric imaging and verification tools and approach real time feedback loops. These tools are improving radiation targeting conformality, and directly increase the therapeutic ratio by reducing dose to normal tissues $[9,10]$ or dose escalation to the target volumes. Advanced computing and optimisation algorithms and raw computing power enable contemporary clinical radiotherapy practice.

A general overview of the various phases of a clinical radiotherapy treatment is shown in figure 1.1. After diagnosis and the treatment prescription, that may include other treatment modalities, functional and anatomical image guidance is used as a basis for the radiotherapy plan. Much care is dedicated to precisely define target and avoidance regions. Usually, multiple radiation dose fractions are delivered to the patient, with relatively small daily doses. The whole process may take several weeks. During the treatment delivery stage, additional image guidance is used for patient positioning during each fraction, and delivered irradiation fields can be measured using an onboard imaging panel, the so called electronic portal imaging device (EPID) [11, 12]. EPID dosimetry enables irradiation verification to revise a treatment if needed using adaptive radiotherapy techniques [13]. This approach enables dose guided radiotherapy (DGRT) and has evolved to time dependent dose verification methods [14]. With the current state of data heavy radiotherapy treatments with high complexity, dedicated record and verify systems are used to orderly gather and store radiation treatment data. 

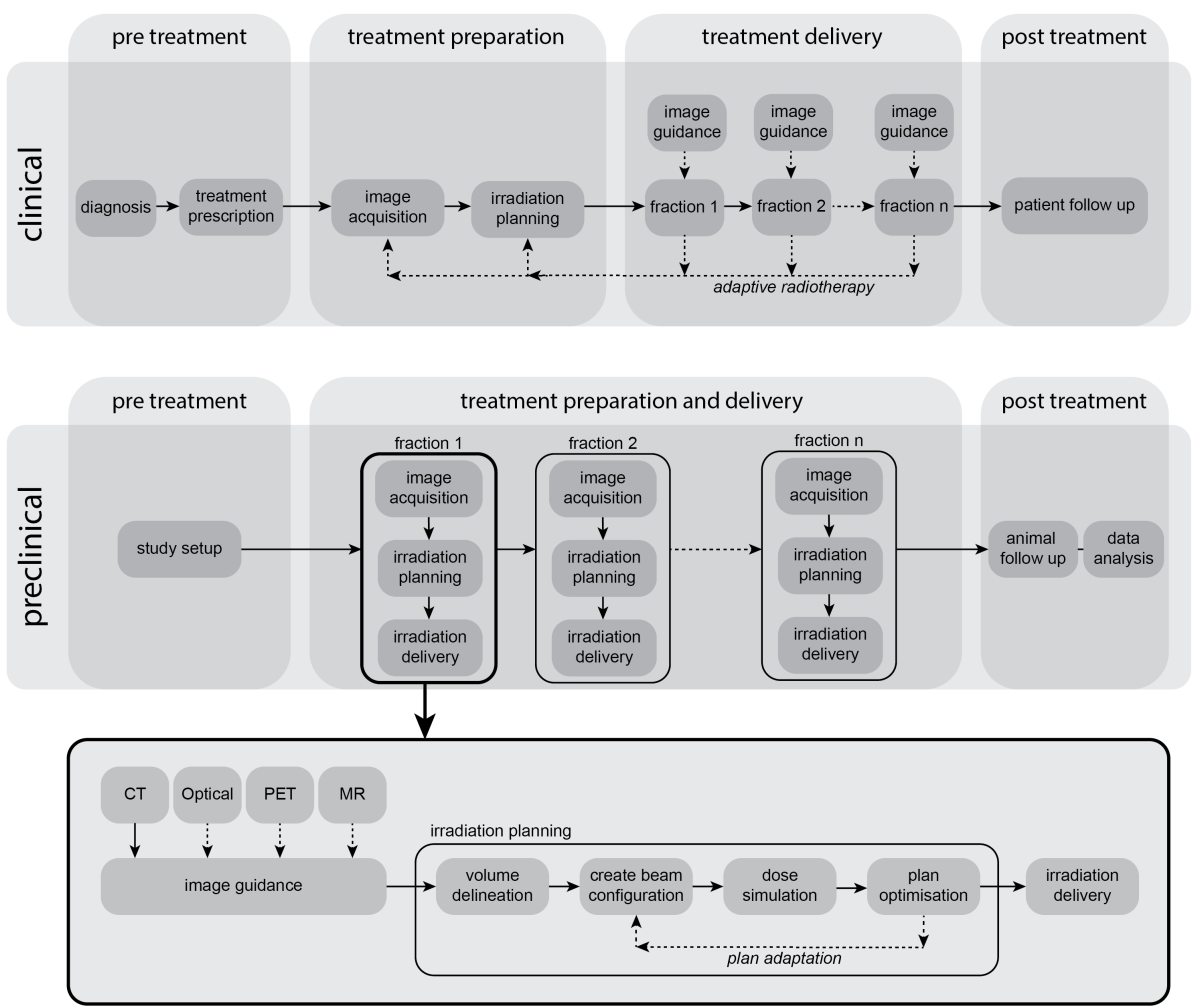

Figure 1.1: The general workflow of a clinical and preclinical radiotherapy treatment course. A clinical course starts with a diagnosis and prescription of the radiation treatment, that can be part of a multi-modal treatment scheme. Pre-treatment imaging is performed for volume of interest identification and creation of the irradiation plan, which is generally delivered during several days to weeks. The irradiation plan is created using a TPS and can take several days to reach final approval. During the treatment delivery phase, verification technologies can indicate the need for treatment adaptations using a feedback loop. A preclinical radiotherapy trajectory starts with the study design. A major difference with its clinical counterpart is that the treatment preparation and delivery phase are combined and carried out during a single session, or multiple consecutive sessions. The animal is imaged in treatment position and the irradiation plan is created, simulated, optimised and delivered, all consecutively within one session. The animal is kept under anaesthesia during this complete phase. Generally, there is only a single or there are only a few irradiation fractions. Although adaptive feedback is possible in the preclinical setting, this is currently not commonly performed. 


\subsection{Preclinical radiotherapy and biological models}

Introducing novel therapeutic strategies such as new anti-cancer agents, treatment schemes, drug combinations, or new or adapted therapy combinations into clinical practice is difficult. The development is costly and time consuming, and clinical trials can be ethically undesirable. In radiotherapy, technological developments have often already progressed considerably by the time the results of a clinical trial become available. This augments the incentive to maximize the relevance of preclinical data to smooth the translation of preclinical efficacy into clinical therapy. The success rate for approval of oncology drugs is lower than for any other disease area [15], the majority of promising preclinical therapies are not replicated in humans [16], and a large portion remains untested [17]. This indicates the need to improve the translation of preclinical data [18]. Failing approaches need to be eliminated as soon as possible and when a promising candidate is found, valuable preclinical research data need to be used for subsequent clinical trial design to e.g. help define a therapeutic window or identify biomarkers for targeting or response quantification.

Preclinical in vitro data need to be tested in vivo to explore cell interaction with the microenvironment, explore biological pathways, and investigate immune responses. However, selecting or creating suitable biological models is challenging and depends on research questions [19]. Already soon after the discovery of x-rays, animals were used to model human radiobiological effects, albeit without translocated tumour cells $[20,21]$. Modern practice involves the development of in vivo models using genetically engineered, patient derived xenografts or spontaneously occurring cancers. Much progress has been made in this area to create biological models with specific knockouts or molecular markers, which has expanded insight in molecular biology [22].

Preclinical animal studies mainly use mice because of similarities to human physiology such as organ systems and genes, knowledge on manipulating genetics to express oncogenes or introduce knock-outs, the relatively ease of breeding, practical handling, and relatively low cost. There are, obviously, substantial differences and therefore limits and often critiques to the use of mouse biology as human model. In comparison with mice, humans are about 3000 times larger in volume, live 30-50 times longer, and have a lower cancer susceptibility [1]. There are other concerns when using murine models such as differences in inflammatory stress response, basic metabolic rates and host immune system, and loss of heterogeneity in cultured cell lines [17].

Traditional animal models mostly use subcutaneous transplantation of tumours on the 
hind leg or flank, which allow for simple irradiation setups such that relevant dose levels can be achieved easily without reaching dose limitations of normal tissues. Subcutaneous models also allow for facile tumour volume estimation using manual calliper measurements, high animal throughputs for strong statistics, and cost effectiveness. Furthermore, preclinical studies are often carried out using a single or only a few radiation fractions, in stark contrast to clinical regimes that can include dozens of fractions. Primary tumours are heterogeneous in their biology, biochemical, and immunological properties, and to e.g. investigate invasiveness or metastatic potential, host-tumour interactions need to be modelled accurately. This importance of the tumour microenvironment led to a growing interest in the use of orthotopic cancer models, which are believed to be more relevant in comparison with heterotopic cancer models [19].

The use of orthotopic cancer models requires more complex irradiation setups. Avoiding limiting normal tissue toxicities while achieving sufficient dose to target volumes becomes a main challenge, similar to clinical radiotherapy practice. In the absence of capable preclinical irradiation hardware, clinical devices with or without modifications or additional ad hoc shielding setups are often used instead. The advancing clinical technologies to enable highly modulated patient irradiations are pushed to their limits to achieve the scale and precision required for preclinical animal irradiations. For example, Trani et al. performed 3D heterogenous PET based dose delivery on the millimetre scale to subcutaneous flank tumours on a rat, using data from a clinical PET-CT scanner for functional imaging based treatment optimisation on a clinical TPS $[23,24]$. Other examples of the use of clinical hardware are the use of a high dose rate brachytherapy systems using an ${ }^{192}$ Ir source [25, 26], an electronic brachytherapy source [27], or the Gamma Knife radiosurgery device [28].

Despite such admirable achievements, the use of clinical equipment has practical and fundamental limitations [29]. The use of MV photon energies results in build-up and penumbra regions of several centimetres, and lack full backscatter conditions at the distal end of media boundaries, resulting in highly non-uniform doses. The innovations in clinical hardware have not been sufficient to meet the requirements for advanced image guided preclinical irradiations [29]. Whereas many imaging modalities have been adapted to the scale and quality required for small animal research in dedicated devices [30], dedicated preclinical irradiation devices have been lagging. 


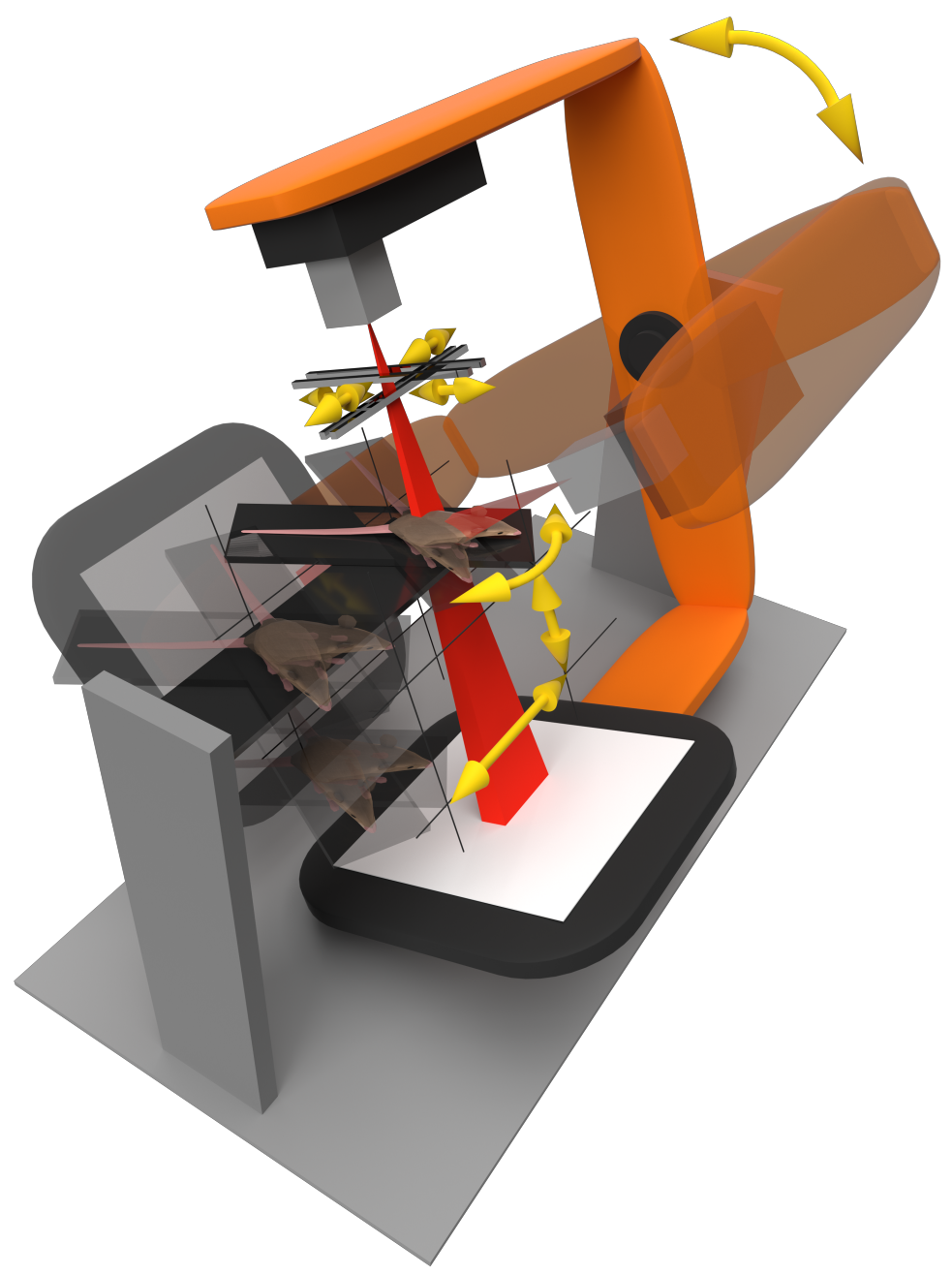

Figure 1.2: A general overview of a micro-IGRT platform. The animal can be positioned using a moveable couch, an orthovoltage $\mathrm{x}$-ray tube is used for irradiation, and image guidance is performed using an EPID. The x-ray tube and EPID are generally placed on opposite sides of a rotatable gantry. More modern hardware iterations are expanding the number of degrees of freedom to include e.g. animal rotation or variable aperture collimation. Onboard imaging capabilities are often cone beam CT and bioluminescence imaging. 


\subsection{Precision small animal image guided radiotherapy}

Several research groups developed and integrated hardware and software to address the lack of experimental irradiation devices downscaled to the murine level. Various approaches have been applied of which one is the extension of simple preclinical orthovoltage $x$-ray cabinets with more sophisticated beam collimation and image guidance [31, 32]. Other groups used a modified micro-CT scanner as basis to enable delivery of therapeutic irradiations. A group from Stanford University retrofitted a commercial micro-CT scanner with an iris-like variable aperture collimator [33-35] and a group from the University of Western Ontario modified a similar micro-CT scanner to deliver gated asymmetrically collimated fields at $140 \mathrm{kVp}$ [36]. Another group at Heidelberg University reported on modifications to an industrial micro-CT unit [37]. A distinct group of institutes followed a path similar to linacs and developed systems that are now known as micro image guided radiotherapy (micro-IGRT) platforms. These platforms generally consist of a high-power industrial x-ray tube and generator, cone beam CT image guidance using an EPID, and the use of robotics for animal and beam positioning. A group from Johns Hopkins University in Baltimore introduced the Small Animal Radiation Research Platform (SARRP) [38, 39] which has been commercialized by Xstrahl Ltd (Surrey, UK), and a group from Princess Margaret Hospital in Toronto developed the X-RAD 225Cx [40] which has been commercialized by Precision X-ray Inc. (North Branford, USA)(PXi). Another group from the Technical University of Dresden developed the Small Animal Image Guided RadioTherapy (SAIGRT) [41] platform, which is not commercially available but is being deployed at other institutes through collaborative efforts. The design of these systems differs but the platforms share many similarities. A general overview of such a micro-IGRT unit is illustrated in figure 1.2. A dual focus $x$-ray tube provides separation of an imaging and therapeutic photon spectrum. Therapeutic doses are delivered using spectra up to $225 \mathrm{kVp}$ at a maximum dose rate of about $4 \mathrm{~Gy} /$ minute, and manually interchangeable collimators are used for beam collimation. Other groups have reported on similar in-house built systems such as a group from the University of Arkansas Medical Sciences that uses a robotic arm [42, 43] and a group from University of Miami School of Medicine that employs a static beam setup [44].

The commercially available platforms have gone through ongoing improvements over the years. For example, the SARRP and X-RAD 225Cx were extended with integrated bioluminescence optical imaging [45, 46], and more advanced collimation and animal positioning systems have been developed. The work of this thesis uses the X-RAD $225 \mathrm{Cx}$ micro-IGRT platform which is installed at the University of Maastricht. The X- 

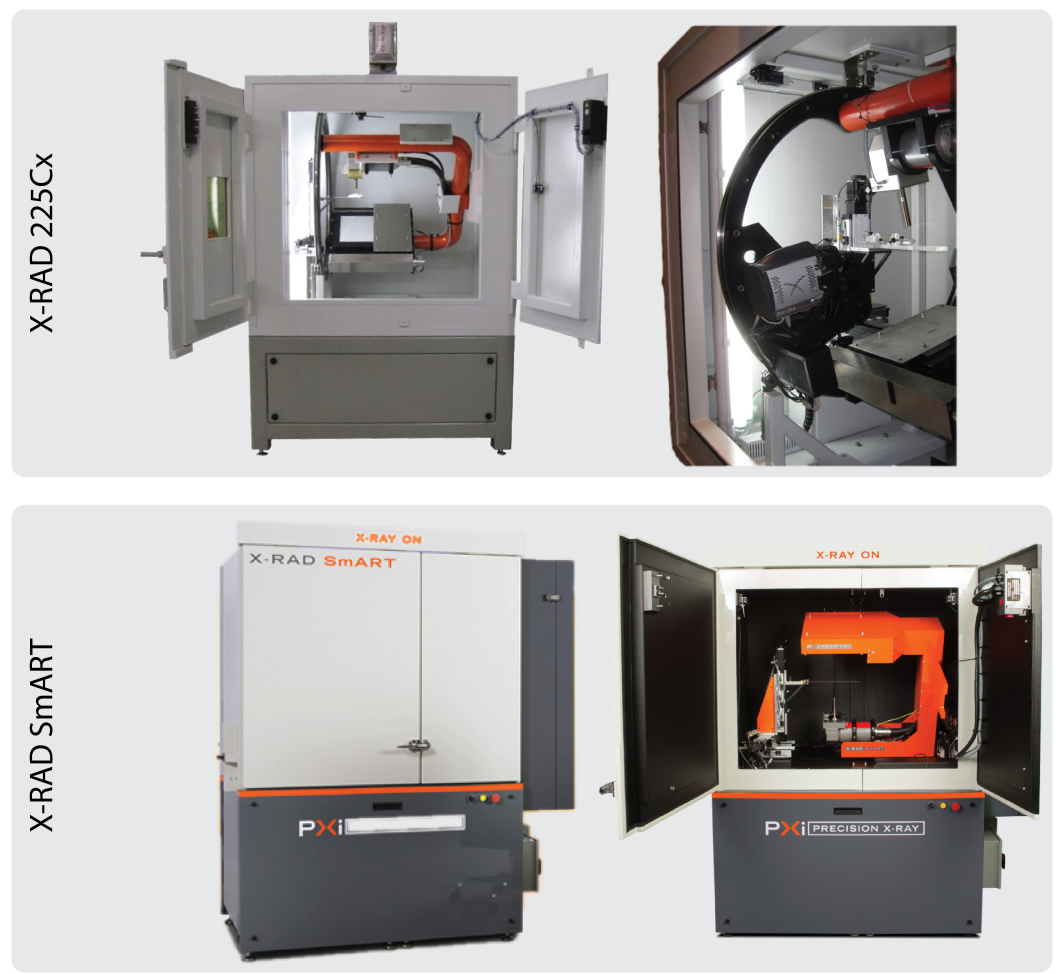

Figure 1.3: Pictures of the commercial micro-IGRT platform manufactured by PXi, of which the first commercial installation occurred in our department at Maastricht University in 2010. The initial version X-RAD 225Cx evolved into the X-RAD SmART.

RAD 225Cx has been described in detail by Clarkson et al. [40], and has evolved into the X-RAD SmART [47], see figure 1.3.

\subsection{Irradiation planning for precision image guided small animal radiotherapy}

The relatively recent development of new biological models and highly capable microIGRT platforms led to the emergence of a completely new radiobiological research field [48, 49]. This unique setting of combined knowledge of biologists, physicists, medical doctors, and engineers enables the exploration of a wide range of research topics. With this evolution, a new unmet need for irradiation planning software arose. Software that enables the exploitation of the full potential of orthotopic biological mod- 
els used in combination with micro-IGRT platforms. These micro-IGRT platforms preferentially use kilovolt $(\mathrm{kV})$ photons instead of megavolt $(\mathrm{MV})$ photons which are most often used in clinical practice [29]. The underlying radiation physics models of a clinical TPS do not support kV photon calculation, and a clinical TPS is not designed for irradiation planning for small animals. This leads to other problems such as radiation planning and optimisation at the required spatial scale, lack of support to incorporate specific preclinical imaging modalities such as bioluminescence data, lack of support to communicate an irradiation plan with micro-IGRT devices, and possible issues with volume of interest delineation, and data and treatment visualization.

The work of this thesis uses Monte Carlo (MC) simulations as dose calculation engine. MC simulations are arguably the golden standard for dose calculations in radiotherapy [50, 51], and have often been used for orthovoltage photon beam calculations [52-54]. MC is a calculation approach that simulates physical processes many times, during which the process outcomes are selected pseudorandom from a statistical distribution. The use of randomness is at the core of this method and used in various other fields such as physics, chemistry, and mathematics. In the case of radiation simulations, millions of individual simulations are used to calculate a dose distribution. Accurate models of the irradiation hardware [55] and irradiation subject are used to simulate the transport of charged and uncharged particles through geometries of different media and densities.

All MC simulations in this thesis were performed using the MC code DOSXYZnrc [56] to calculate dose in phantoms with rectilinear voxels, BEAMnrc $[57,58]$ to model the irradiator, and an analytical model for fast generation of beam models [59]. DOSXYZnrc and BEAMnrc are user applications built on top of the general purpose EGSnrc code system which is currently maintained by the National Research Council of Canada $[60,61]$. EGSnrc is an evolution of the EGS4 code system [62], which in turn is an evolution of many versions and programs ultimately dating back to the 1960's when punch cards were used to store the respective code systems. The development of the predecessors of the EGS codes were motivated by the construction of the Stanford Linear Accelerator (SLAC) to model high-energy electron beams [63]. The OMEGA project in the early 90's [64], led to the development of the user code BEAM, the predecessor of BEAMnrc, that could be used to model linacs, and boosted the field of medical physics tremendously.

Great progress has been made to minimize CPU usage by using variance reduction [65] and condensed history techniques [66]. Still, the required calculation time of MC 
simulations was often unacceptable in the past, but practical applicability of MC simulation for radiotherapy planning has become achievable for various reasons. With the increase of single CPU computing power, the advent of multi core computing, and decreasing cost of CPU power and data storage, computing power has seen immense growth the past decades. The introduction of programmable shaders and floatingpoint support on graphics processors in the early 2000's made general-purpose computing on graphical processing units (GPGPU) possible. More recently, MC codes are being developed to harness the parallel nature of graphics processing.

\subsection{Outline}

In summary, the motivation for the work of this thesis originated from the development of new biological models and micro-IGRT systems, which led to a new research field in radiobiological studies. Any aspect of radiobiology that involves interaction of the tumour with its surroundings could potentially benefit from orthotopic versus heterotopic animal models, and targeted localized irradiations can facilitate normal tissue toxicity studies. The maximisation of the relevance of preclinical in vivo research data is key to reduce the number of failing approaches, to design potentially successful clinical trials, and reduce overall animal burden. To fully exploit the potential of this new research area, capable radiation planning methods and algorithms for small animal radiotherapy are required. In the next chapter, a review of specific issues and treatment planning for preclinical precision irradiation of small animals is presented. To serve an unmet need, a novel treatment planning system dedicated to micro-IGRT systems was developed and validated which is described in chapter 3 . The advancements of this MC based treatment planning system to include beam-on time optimisation and conformal dose painting irradiations are described in chapters 4 and 5 . In chapter 6 the developed techniques are demonstrated with localized preclinical irradiations using an orthotopic glioblastoma model in mice, and chapter 7 closes with a general discussion, future perspectives, and conclusions. 


\section{References}

1. Rangarajan A, Weinberg RA. Opinion: Comparative biology of mouse versus human cells: modelling human cancer in mice. Nat. Rev. Cancer 2003; 3: 952-959. doi: 10.1038/nrc1235.

2. Centraal Bureau voor de Statistiek. Overledenen; belangrijke doodsoorzaken (korte lijst), leeftijd, geslacht. Available from: https://opendata.cbs.nl/statline/\#/CBS/nl/dataset/7052_95/table?dl=E608.

3. Bray F, Jemal A, Grey N, Ferlay J, Forman D. Global cancer transitions according to the Human Development Index (2008-2030): a population-based study. Lancet Oncol. 2012; 13: 790-801. doi: 10.1016/S1470-2045(12)70211-5.

4. Atun R, Jaffray DA, Barton MB, Bray F, Baumann M, Vikram B, Hanna TP, Knaul FM, Lievens Y, Lui TYM, Milosevic M, O'Sullivan B, Rodin DL, Rosenblatt E, Van Dyk J, Yap ML, Zubizarreta E, Gospodarowicz M. Expanding global access to radiotherapy. Lancet Oncol. 2015; 16: 1153-1186. doi: 10.1016/S1470-2045(15)00222-3.

5. Bloom DE, Cafiereo ET, Jane-Llopis E, Abrahams-Gessel S, Bloom LR, Fathima S, Feigl AB, Gaziano T, Mowafi M, Pandya A, Prettner K, Rosenberg L, Seligman B, Stein A, \& Weinstein C. The Global Economic Burden of Non-communicable Diseases. 2011; Available from: http://www3.weforum.org/docs/WEF_Harvard_HE_GlobalEconomicBurdenNonCommunicableDiseases_MethodologicalAppendix_2011.pdf.

6. Pastink A, Eeken JC, Lohman PH. Genomic integrity and the repair of double-strand DNA breaks. Mutat. Res. 2001; 480-481: 37-50.

7. Purdy JA. Intensity-modulated radiation therapy. Int. J. Radiat. Oncol. Biol. Phys. 1996; 35 : 845-846.

8. Teoh M, Clark $\mathrm{CH}$, Wood K, Whitaker S, Nisbet A. Volumetric modulated arc therapy: a review of current literature and clinical use in practice. Br. J. Radiol. 2011; 84: 967-996. doi: $10.1259 / \mathrm{bj} r / 22373346$.

9. Staffurth J, Radiotherapy Development Board. A review of the clinical evidence for intensitymodulated radiotherapy. Clin. Oncol. R. Coll. Radiol. G. B. 2010; 22: 643-657. doi: 10.1016/j.clon.2010.06.013.

10. Veldeman L, Madani I, Hulstaert F, De Meerleer G, Mareel M, De Neve W. Evidence behind use of intensity-modulated radiotherapy: a systematic review of comparative clinical studies. Lancet Oncol. 2008; 9: 367-375. doi: 10.1016/S1470-2045(08)70098-6.

11. Boyer AL, Antonuk L, Fenster A, Van Herk M, Meertens H, Munro P, Reinstein LE, Wong J. A review of electronic portal imaging devices (EPIDs). Med. Phys. 1992; 19: 1-16. doi: 10.1118/1.596878.

12. van Elmpt W, McDermott L, Nijsten S, Wendling M, Lambin P, Mijnheer B. A literature review of electronic portal imaging for radiotherapy dosimetry. Radiother. Oncol. J. Eur. Soc. Ther. Radiol. Oncol. 2008; 88: 289-309. doi: 10.1016/j.radonc.2008.07.008.

13. Persoon LCGG. Novel in-treatment dose verification methods for adaptive radiotherapy. 2016;

14. Podesta M. Time dependent verification of dynamic external beam radiotherapy. 2016;

15. Hay M, Thomas DW, Craighead JL, Economides C, Rosenthal J. Clinical development success rates for investigational drugs. Nat. Biotechnol. 2014; 32: 40-51. doi: 10.1038/nbt.2786.

16. Gould SE, Junttila MR, de Sauvage FJ. Translational value of mouse models in oncology drug development. Nat. Med. 2015; 21: 431-439. doi: 10.1038/nm.3853.

17. Koontz BF, Verhaegen F, De Ruysscher D. Tumour and normal tissue radiobiology in mouse 
models: how close are mice to mini-humans? Br. J. Radiol. 2017; 90: 20160441. doi: 10.1259/bjr.20160441.

18. Coleman CN, Higgins GS, Brown JM, Baumann M, Kirsch DG, Willers H, Prasanna PGS, Dewhirst MW, Bernhard EJ, Ahmed MM. Improving the Predictive Value of Preclinical Studies in Support of Radiotherapy Clinical Trials. Clin. Cancer Res. Off. J. Am. Assoc. Cancer Res. 2016; 22: 3138-3147. doi: 10.1158/1078-0432.CCR-16-0069.

19. Killion JJ, Radinsky R, Fidler IJ. Orthotopic models are necessary to predict therapy of transplantable tumors in mice. Cancer Metastasis Rev. 1998; 17: 279-284.

20. Regaud C, Nogier T. Sterilization rontgenienne totale et definitive, sans radiodermite, des testicules du Belier adulte: conditions de sa realisation. Compt Rend Soc Biol 1911; 70: 202-203.

21. Mottram JC. A Factor of Importance in the Radio Sensitivity of Tumours. Br. J. Radiol. 1936; 9 : 606-614. doi: 10.1259/0007-1285-9-105-606.

22. Kerbel RS. A Decade of Experience in Developing Preclinical Models of Advancedor Early-Stage Spontaneous Metastasis to Study Antiangiogenic Drugs, Metronomic Chemotherapy, and the Tumor Microenvironment. Cancer J. Sudbury Mass 2015; 21: 274-283. doi: 10.1097/PPO.0000000000000134.

23. Trani D, Reniers B, Persoon L, Podesta M, Nalbantov G, Leijenaar RTH, Granzier M, Yaromina A, Dubois L, Verhaegen F, Lambin P. What Level of Accuracy Is Achievable for Preclinical Dose Painting Studies on a Clinical Irradiation Platform? Radiat. Res. 2015; 183: 501-510. doi: 10.1667/RR13933.1.

24. Trani D, Yaromina A, Dubois L, Granzier M, Peeters SGJA, Biemans R, Nalbantov G, Lieuwes N, Reniers B, Troost EEGC, Verhaegen F, Lambin P. Preclinical Assessment of Efficacy of Radiation Dose Painting Based on Intratumoral FDG-PET Uptake. Clin. Cancer Res. Off. J. Am. Assoc. Cancer Res. 2015; 21: 5511-5518. doi: 10.1158/1078-0432.CCR-15-0290.

25. Stojadinovic S, Low DA, Hope AJ, Vicic M, Deasy JO, Cui J, Khullar D, Parikh PJ, Malinowski KT, Izaguirre EW, Mutic S, Grigsby PW. MicroRT-small animal conformal irradiator. Med. Phys. 2007; 34: 4706-4716. doi: 10.1118/1.2799887.

26. Grams MP, Wilson ZC, Sio TT, Beltran CJ, Tryggestad EJ, Gupta SK, Blackwell CR, McCollough KP, Sarkaria JN, Furutani KM. Design and characterization of an economical (192)Ir hemi-brain small animal irradiator. Int. J. Radiat. Biol. 2014; 90: 936-942. doi: 10.3109/09553002.2014.922719.

27. Rodriguez $M$, Jeraj $R$. Design of a radiation facility for very small specimens used in radiobiology studies. Phys. Med. Biol. 2008; 53: 2953-2970. doi: 10.1088/0031-9155/53/11/013.

28. DesRosiers C, Mendonca MS, Tyree C, Moskvin V, Bank M, Massaro L, Bigsby RM, CaperallGrant A, Valluri S, Dynlacht JR, Timmerman R. Use of the Leksell Gamma Knife for localized small field lens irradiation in rodents. Technol. Cancer Res. Treat. 2003; 2: 449-454. doi: 10.1177/153303460300200510.

29. Verhaegen F, Granton P, Tryggestad E. Small animal radiotherapy research platforms. Phys. Med. Biol. 2011; 56: R55-83. doi: 10.1088/0031-9155/56/12/R01.

30. Kagadis GC, Loudos G, Katsanos K, Langer SG, Nikiforidis GC. In vivo small animal imaging: current status and future prospects. Med. Phys. 2010; 37: 6421-6442. doi: 10.1118/1.3515456.

31. Pidikiti R, Stojadinovic S, Speiser M, Song KH, Hager F, Saha D, Solberg TD. Dosimetric characterization of an image-guided stereotactic small animal irradiator. Phys. Med. Biol. 2011; 56: 2585-2599. doi: 10.1088/0031-9155/56/8/016.

32. Song KH, Pidikiti R, Stojadinovic S, Speiser M, Seliounine S, Saha D, Solberg TD. An x-ray 
image guidance system for small animal stereotactic irradiation. Phys. Med. Biol. 2010; 55: 7345-7362. doi: 10.1088/0031-9155/55/23/011.

33. Zhou H, Rodriguez M, van den Haak F, Nelson G, Jogani R, Xu J, Zhu X, Xian Y, Tran PT, Felsher DW, Keall PJ, Graves EE. Development of a micro-computed tomography-based image-guided conformal radiotherapy system for small animals. Int. J. Radiat. Oncol. Biol. Phys. 2010; 78: 297-305. doi: 10.1016/j.jijrobp.2009.11.008.

34. Graves EE, Zhou H, Chatterjee R, Keall PJ, Gambhir SS, Contag CH, Boyer AL. Design and evaluation of a variable aperture collimator for conformal radiotherapy of small animals using a microCT scanner. Med. Phys. 2007; 34: 4359-4367. doi: 10.1118/1.2789498.

35. Bazalova M, Zhou H, Keall PJ, Graves EE. Kilovoltage beam Monte Carlo dose calculations in submillimeter voxels for small animal radiotherapy. Med. Phys. 2009; 36: 4991-4999. doi: 10.1118/1.3238465.

36. Jensen MD, Hrinivich WT, Jung JA, Holdsworth DW, Drangova M, Chen J, Wong E. Implementation and commissioning of an integrated micro-CT/RT system with computerized independent jaw collimation. Med. Phys. 2013; 40: 081706. doi: 10.1118/1.4812422.

37. Felix MC, Glatting G, Giordano FA, Brockmann MA, Wenz F, Fleckenstein J. Collimator optimization for small animal radiation therapy at a micro-CT. Z. Med. Phys. 2017; 27: 56-64. doi: 10.1016/j.zemedi.2016.05.003.

38. Wong J, Armour E, Kazanzides P, lordachita I, Tryggestad E, Deng H, Matinfar M, Kennedy C, Liu Z, Chan T, Gray O, Verhaegen F, McNutt T, Ford E, DeWeese TL. High-resolution, small animal radiation research platform with $\mathrm{x}$-ray tomographic guidance capabilities. Int. J. Radiat. Oncol. Biol. Phys. 2008; 71: 1591-1599. doi: 10.1016/j.jijobp.2008.04.025.

39. Matinfar M, Ford E, lordachita I, Wong J, Kazanzides P. Image-guided small animal radiation research platform: calibration of treatment beam alignment. Phys. Med. Biol. 2009; 54: 891-905. doi: 10.1088/0031-9155/54/4/005.

40. Clarkson R, Lindsay PE, Ansell S, Wilson G, Jelveh S, Hill RP, Jaffray DA. Characterization of image quality and image-guidance performance of a preclinical microirradiator. Med. Phys. 2011; 38: 845-856. doi: 10.1118/1.3533947.

41. Tillner F, Thute P, Löck $S$, Dietrich A, Fursov A, Haase R, Lukas M, Rimarzig B, Sobiella M, Krause M, Baumann M, Bütof R, Enghardt W. Precise image-guided irradiation of small animals: a flexible non-profit platform. Phys. Med. Biol. 2016; 61: 3084-3108. doi: 10.1088/00319155/61/8/3084.

42. Moros E, Sharma S, Corry P, Chao M, Griffin R, Mihaylov I, Penagaricano J. TU-C-BRD-03: An Integrated Robotic-Based Irradiation System for Small Animal Research. Med. Phys. 2009; 36: 2720-2720. doi: 10.1118/1.3182321.

43. Sharma S, Narayanasamy G, Przybyla B, Webber J, Boerma M, Clarkson R, Moros EG, Corry PM, Griffin RJ. Advanced Small Animal Conformal Radiation Therapy Device. Technol. Cancer Res. Treat. 2017; 16: 45-56. doi: 10.1177/1533034615626011.

44. Sha H, Udayakumar TS, Johnson PB, Dogan N, Pollack A, Yang Y. An image guided small animal stereotactic radiotherapy system. Oncotarget 2016; 7: 18825-18836. doi: 10.18632/oncotarget.7939.

45. Weersink RA, Ansell S, Wang A, Wilson G, Shah D, Lindsay PE, Jaffray DA. Integration of optical imaging with a small animal irradiator. Med. Phys. 2014; 41: 102701. doi: 10.1118/1.4894730.

46. Zhang B, Wang KK-H, Yu J, Eslami S, lordachita I, Reyes J, Malek R, Tran PT, Patterson MS, Wong JW. Bioluminescence Tomography-Guided Radiation Therapy for Preclinical Research. 
Int. J. Radiat. Oncol. Biol. Phys. 2016; 94: 1144-1153. doi: 10.1016/j.jrobp.2015.11.039.

47. Precision X-Ray: X-RAD SmART, Small Animal Image Guided Irradiation System. Available from: http://www.pxinc.com/products/small-animal-igrt-platform/x-rad-smart-small-animalradiation-therapy-precision-xray-branford-connecticut/.

48. Butterworth KT, Prise KM, Verhaegen F. Small animal image-guided radiotherapy: status, considerations and potential for translational impact. Br. J. Radiol. 2015; 88: 20140634. doi: 10.1259/bjr.20140634.

49. Tillner $F$, Thute $P$, Bütof $R$, Krause $M$, Enghardt W. Pre-clinical research in small animals using radiotherapy technology-a bidirectional translational approach. Z. Med. Phys. 2014; 24: 335-351. doi: 10.1016/j.zemedi.2014.07.004.

50. Ali ESM, Rogers DWO. Benchmarking EGSnrc in the kilovoltage energy range against experimental measurements of charged particle backscatter coefficients. Phys. Med. Biol. 2008; 53: 1527-1543. doi: 10.1088/0031-9155/53/6/002.

51. Seco J, Verhaegen F. Monte Carlo Techniques in Radiation Therapy. 2013;

52. Penchev P, Mäder U, Fiebich M, Zink K. Design and evaluation of a Monte Carlo based model of an orthovoltage treatment system. Z. Med. Phys. 2015; 25: 341-352. doi: 10.1016/j.zemedi.2015.05.005.

53. Chow JCL, Grigorov GN. Effect of the bone heterogeneity on the dose prescription in orthovoltage radiotherapy: A Monte Carlo study. Rep. Pract. Oncol. Radiother. J. Gt. Cancer Cent. Poznan Pol. Soc. Radiat. Oncol. 2011; 17: 38-43. doi: 10.1016/j.rpor.2011.09.001.

54. Monti di Sopra F, Keall P, Beckham W. Comparing dose calculation algorithms for an orthovoltage beam in a bone phantom. Australas. Phys. Eng. Sci. Med. 1998; 21: 148-151.

55. Granton PV, Podesta M, Landry G, Nijsten S, Bootsma G, Verhaegen F. A combined dose calculation and verification method for a small animal precision irradiator based on onboard imaging. Med. Phys. 2012; 39: 4155-4166. doi: 10.1118/1.4725710.

56. Walters B, Kawrakow I, Rogers DWO. DOSXYZnrc Users Manual. 2005; 132. Available from: https://nrc-cnrc.github.io/EGSnrc/doc/pirs794-dosxyznrc.pdf.

57. Rogers DW, Faddegon BA, Ding GX, Ma CM, We J, Mackie TR. BEAM: a Monte Carlo code to simulate radiotherapy treatment units. Med. Phys. 1995; 22: 503-524. doi: 10.1118/1.597552.

58. Rogers DWO, Walters B, Kawrakow I. BEAMnrc Users Manual. 2015; 290. Available from: https://nrc-cnrc.github.io/EGSnrc/doc/pirs509a-beamnrc.pdf.

59. Granton PV, Verhaegen F. On the use of an analytic source model for dose calculations in precision image-guided small animal radiotherapy. Phys. Med. Biol. 2013; 58: 3377. doi: 10.1088/0031-9155/58/10/3377.

60. Kawrakow I, Rogers DWO, Tessier F, Walters B. The EGSnrc Code System: Monte Carlo Simulation of Electron and Photon Transport. 2011; 323 . Available from: https://nrc-cnrc.github.io/EGSnrc/doc/pirs701-egsnrc.pdf.

61. National Research Council Canada. EGSnrc: software tool to model radiation transport - National Research Council Canada. 2013; Available from: https://www.nrc-cnrc.gc.ca/eng/solutions/advisory/egsnrc_index.html.

62. Nelson WR, Hirayama H, Rogers DWO. THE EGS4 CODE SYSTEM. 1985; 206. Available from: http://www.slac.stanford.edu/cgi-wrap/getdoc/slac-r-265.pdf.

63. Ford RL, Nelson WR. THE EGS CODE SYSTEM: COMPUTER PROGRAMS FOR THE MONTE CARLO SIMULATION OF ELECTROMAGNETIC CASCADE SHOWERS (VERSION 3). 1978; 
64. Mackie TR, Kubsad SS, Reckwerdt PJ, Kinsella TJ, Bielajew AF, Rogers DWO. The OttawaMadison Electron Gamma Algorithm (OMEGA) Project: feasibility of two Monte Carlo techniques. 250-253.

65. Walters B. Application of efficient Monte Carlo photon beam simulations to dose calculations in voxellized human phantoms. 2017;

66. Kawrakow I. Accurate condensed history Monte Carlo simulation of electron transport. I. EGSnrc, the new EGS4 version. Med. Phys. 2000; 27: 485-498. doi: 10.1118/1.598917. 



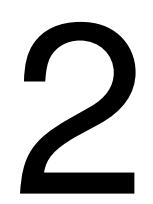

A review of treatment planning for precision image guided photon beam preclinical animal radiation studies

authors

Frank Verhaegen, Stefan J van Hoof, Patrick V Granton, Daniela Trani adapted from Zeitschrift für Medizinische Physik 2014; 24: 323-334 


\section{Chapter 2}

\section{Abstract}

Recently, precision irradiators integrated with a high-resolution CT imaging device became available for preclinical studies. These research platforms offer significant advantages over older generations of animal irradiators in terms of precision and accuracy of image guided radiation targeting. These platforms are expected to play a significant role in defining experiments that will allow translation of research findings to the human clinical setting. In the field of radiotherapy, but also others such as neurology, the platforms create unique opportunities to explore e.g. the synergy between radiation and drugs or other agents. To fully exploit the advantages of this new technology, accurate methods are needed to plan the irradiation and to calculate the three-dimensional radiation dose distribution in the specimen. To this end, dedicated treatment planning systems are needed. In this review we will discuss specific issues for precision irradiation of small animals, we will describe the workflow of animal treatment planning, and we will examine several dose calculation algorithms (factorization, superposition-convolution, Monte Carlo simulation) used for animal irradiation with kilovolt photon beams. Issues such as dose reporting methods, photon scatter, tissue segmentation and motion will also be discussed briefly. 


\subsection{Introduction}

The technical capabilities in human radiotherapy have reached a high level of sophistication with e.g. intensity modulated radiotherapy and volumetric modulated arc therapy whereby the photon fluence is modulated while a medical accelerator irradiates the tumour with a crossfire of beams from several directions [1, 2]. Radiation dose distributions can now be sculpted intricately with techniques such as dose painting e.g. to preferentially target hypoxic tumour regions [3]. This degree of sophisticated beam delivery requires an equally complex level of planning and dose calculation which is nowadays available in clinical treatment planning systems (TPS). Arguably the next progress in radiation delivery comes in the form of hadron beams such as protons and carbon ions [4] where also sophisticated treatment planning methods are being developed [5]. Most progress in radiotherapy, real or perceived, has come from technological developments, and not from knowledge derived e.g. from animal irradiation experiments [6]. The latter were mostly performed with irradiation technology which bears little resemblance to modern radiotherapy equipment. The recent development of many animal tumour models has enabled the detailed study of the tumour micro environment and the interaction of radiation with tumours. Improved models of normal tissue response are also needed to assess optimal radiotherapy strategies. The recent literature [6-8] has described some novel technology which, for the first time, allows precision image guided radiotherapy for preclinical studies in radiotherapy. It combines narrow radiation beams of photons which may be aimed precisely at tumorous or healthy tissues with the aid of x-ray imaging equipment. It is expected that this new technology may lead to meaningful translation of novel forms of cancer therapy, e.g. by exploiting the synergy between radiation and drugs or other agents. In a recent review paper on the novel animal technology [6] it was explained why small animal precision irradiation with photons is preferably done with kilovolt $(\mathrm{kV})$ instead of megavolt (MV) photons. The main rationale is to avoid extensive dose build-up regions near medium interfaces, and to avoid wide beam penumbras, sometimes encompassing the whole animal. Many animal studies done in the past didn't employ a TPS and usually no imaging was available. Using a human TPS for animal studies is not really advisable, at least not without precautions. Human TPS employ calculation models unsuitable for small beams $(<3 \mathrm{~cm}$ field size). One of the few animal studies accurately modelling small beams of high energy photon beams with a dedicated TPS is [9] where rats were irradiated with small beams of ${ }^{60} \mathrm{Co}$ photons from a GammaKnife device. A human TPS may not be suited to handle the animal voxel phantoms from e.g. a CT or MRI scan with commonly a large number of very small voxels. There is also no human TPS that can reliably calculate dose distributions in 
heterogeneous phantoms irradiated by kV photon energies. Recently, dedicated photon dose calculation methods for small animals have been proposed from relatively simple pencil beam type calculations to Monte Carlo simulations. The latter has the potential of being the most accurate dose calculation technique available for a wide range of conditions [10], provided accurate models are used. This review discusses several of these methods with an emphasis on the technical capabilities and not on treatment planning studies themselves. Sometimes precision proton [11] or hadron beams [12] are used for preclinical studies; treatment planning for these is not included in this review. Treatment planning for radiotherapy for companion animals is also excluded from the review.

\subsection{Issues for small animal radiation dose calculation}

In the currently commercially available precision small animal irradiators a photon spectrum of about $225 \mathrm{kV}$ is used with collimators shaping small beams with dimensions of $0.5 \mathrm{~mm}$ up to a few centimetres. Some in-house developed systems use other photon energies. In the commercial devices the x-ray tube is mounted to allow irradiation of the specimen from multiple sides, and also to enable arced beams. The same x-ray tube, with a lower tube voltage (typically 50-80 kV) and a wider field of view typically encompassing a whole animal, is used to acquire a cone beam CT (CBCT) image of the specimen. The CBCT imaging panel typically has a high spatial resolution of about 100-200 $\mu \mathrm{m}$ (compare to 1-3 $\mathrm{mm}$ in human CT scanners). The resulting 3D CBCT images may have a very large number of small voxels, easily in the tens of millions. Storing a large number of these images in studies involving many animals with each possibly undergoing several imaging sessions, may require special consideration for data archiving.

In contrast to the MV energy range of photons where Compton scatter is the only relevant photon interaction process, for $\mathrm{kV}$ photons the photo-electric effect becomes increasingly important at lower energies. Whereas the probability of the Compton effect for different tissues depends only on the electron density, the probability of photoelectric effect depends very strongly on the effective atomic number $Z^{3-4}$ of the tissues. Since $Z$ ranges from about 6-14 for human tissues, the probability for photoelectric effect differs greatly between the tissues. Figure 2.1 shows that the mass energy absorption coefficients [13] for various human tissues [14] differ significantly from water. This quantity is closely related to absorbed radiation dose. Cortical bone and skeletal muscle, which often occur in each other's proximity anatomically, differ by 

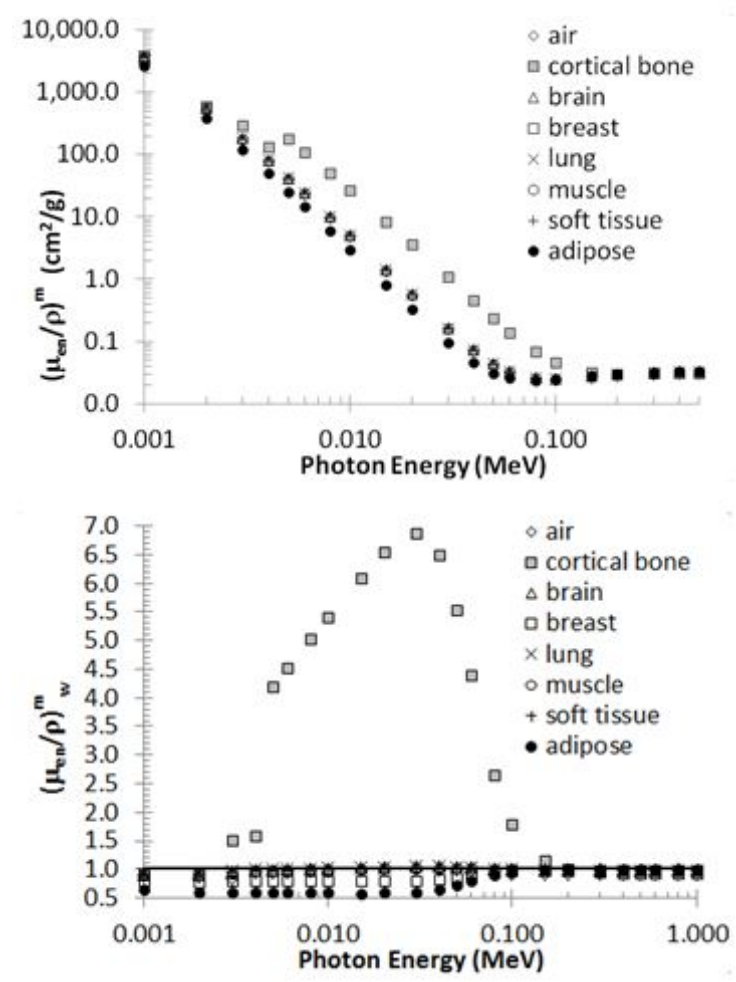

Figure 2.1: Photon mass energy absorption coefficients (top) and relative (to water) mass energy absorption coefficients (bottom) for various (human) media of interest. 

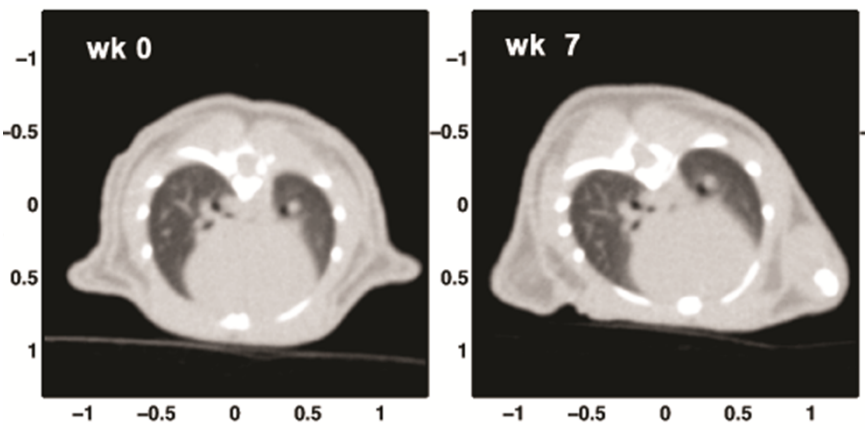

Figure 2.2: CBCT image of the onboard scanner from a X-RAD 225Cx irradiator (PXi, North Branford, USA). Axial sections of the same mouse are shown at different time points after irradiation (week 0 and week 7).

more than a factor six in their energy absorption around $30 \mathrm{keV}$. Accurate dose calculation may require defining several bone types in this case. Even media such as soft tissue and adipose differ significantly in energy absorption. In MV photon energies dose calculations are much less sensitive to the tissue compositions. A potentially large source of uncertainty is that actual animal tissues appear to be unknown in their composition. However, it is clear that assigning water to all voxels will lead to significant over- or underestimation of the absorbed dose in animals. The current practice in animal dose calculation is therefore to assign human tissue types to the voxels, but with the density derived from the CBCT or CT image. Almost certainly, anything resembling human cortical bone is not present in large regions in small animals, and certainly not with the densities as they occur in humans. From this discussion it is also clear that to avoid large uncertainties with heterogeneous dose distributions due to tissue absorption, photon energies above $100 \mathrm{keV}$ should be used preferentially for small animal radiotherapy research. On the other hand, one may wish to irradiate with lower photon energies to e.g. assess effects of relative biological effectiveness (RBE) in animals, which is known to be enhanced at lower photon energies [15]. In this case one should be aware of the dose uncertainties at lower energies. A state of the art TPS for preclinical studies should therefore be able to provide information on the photon spectrum in various animal tissues, thereby allowing estimates of RBE. 


\subsection{Imaging information for treatment planning}

\subsubsection{CT imaging}

Chief among imaging modalities for human radiotherapy planning is CT imaging, with other modalities such as PET and MRI used complementary. This is mainly because information on electron density is needed for the dose calculation. The same holds for small animal dose calculations but in addition tissue types need to be identified for reasons explained in the previous section. The required spatial resolution is typically of the order of $100-200 \mu \mathrm{m}$ to ensure a sufficient volumetric accuracy to image anatomical structures, tumours or subparts thereof. Modern commercial small animal irradiators have an onboard CBCT x-ray imaging device, but equally well could the planning process be based on an externally acquired CT image. Figure 2.2 shows CBCT images of the same mouse at different time points in a longitudinal study [16]. Obviously, the position of the animal can be different during different imaging sessions, depending on the rigorousness of the animal fixation procedure. If also reirradiation of the same target in the specimen with precise beams is involved, care has to be taken to adjust the treatment plan accordingly. This may also involve using image registration techniques.

In human radiotherapy CBCT images usually have a significantly lower imaging quality than $\mathrm{CT}$ images, and are therefore known to potentially hamper the quality of dose calculations [17]. For small animal CBCT images this is less of an issue due to the very limited number of scattered photons contributing to the image formation which degrades the image quality in human applications. On the other hand, the small voxels in animals necessitate a high radiation dose (up to about 0.2-0.4 Gy per exposure [18]) to reach acceptable noise levels. The images in figure 2.2 exhibit high contrast, allow identification of many structures and are relatively free from CT artefacts. Further research into improving small animal CBCT imaging may improve e.g. auto segmentation of structures and the accuracy of the dose calculation since this depends strongly on correct tissue type identification.

The typical workflow for treatment planning for small animals starts with the acquisition of a 3D CBCT (or CT) image, consisting of many small voxels. The specimen then remains on the stage with as little motion as possible, while the CT image reconstruction occurs (this requires about 1 minute). To perform a dose calculation the Hounsfield units $(\mathrm{HU})$ of every voxel need to be assigned a density. This is performed by acquiring an image of a calibration phantom with tissue-equivalent inserts of known densities (e.g. the Model 467; Gammex RMI, Middleton, WI) to derive a HU 


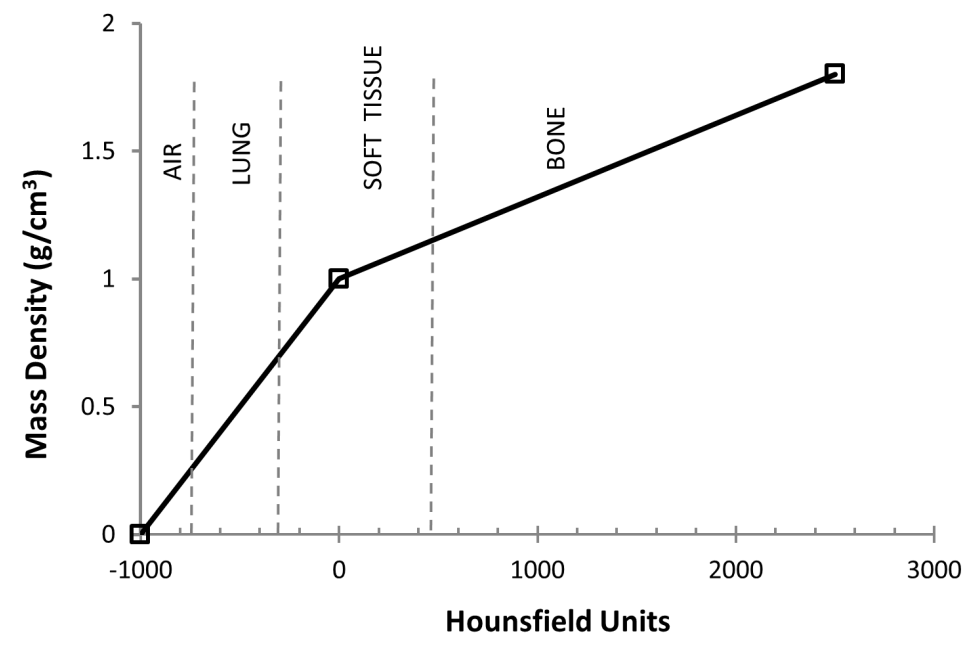

Figure 2.3: Typical HU vs mass density calibration curve, based on a calibration phantom. Only 3 calibration media were used (air at $-1000 \mathrm{HU}$, water at $0 \mathrm{HU}$ and bone equivalent material at $2500 \mathrm{HU}$ ). Four different media will be assigned to the voxels in this scheme (air, lung, soft tissue and bone).

versus density calibration curve. The density could be either mass density or electron density, depending on the requirements of the used dose calculation algorithm (between both quantities there exists a simple relationship for human tissues and their numerical differences are small, except for lung [19]).

Figure 2.3 depicts a typical HU-mass density curve obtained from a calibration procedure with only 3 calibration materials in a phantom. A piecewise bilinear curve was fitted through these points. Mass densities can then be assigned to every voxel. The calibration curve depends on the $\mathrm{kV}$-filter combination (i.e. the radiation quality) so when animals are imaged with different imaging protocols multiple calibrations have to be performed. Many types of dose calculation algorithms, e.g. Monte Carlo radiation transport simulations, need additional information in addition to voxel densities, such as tissue type. A possible way to assign tissues to voxels is to subdivide the whole $\mathrm{HU}$ range into intervals that belong to the same tissue (figure 2.3). This procedure adds a degree of arbitrariness to the voxel conversion. Other methods to assign tissue types rely on dual energy $\mathrm{CT}$ imaging [20]. This form of imaging allows extraction of more information from the specimen compared to single energy CT imaging, e.g. the atomic number. This may support the correct identification of tissue types. In addition to correct tissue and density assignment one should also be aware of the imaging dose to the animals [21] which can significantly contribute towards the total radiation dose, 
especially in longitudinal studies involving repeated imaging. In human radiotherapy a common coordinate system between imaging and treatment rooms is ensured by the use of laser alignment systems on the imaging devices and accelerators, and of robust fiducial systems (markers or frames attached to the patient) or direct imaging of patient structures. In animal radiation studies a similar issue is encountered, but the required accuracy is even higher. If an onboard CBCT imager is used, then the image and the irradiation space are already in the same coordinate system. In case a separate CT imager is used, or another imaging device is employed, a procedure to ensure a common system of coordinates is needed. This may be done e.g. through fiducial markers attached to the animal or to its carrier in combination with some form of imaging. This is a non-trivial issue which will determine the irradiation accuracy to a large extent. Robust animal fixation systems are required to avoid compromising the quality of precision irradiation studies. In radiotherapy images are also highly standardized via the DICOM (Digital Imaging and Communications in Medicine) imaging standard, which is largely absent in the small animal research community.

\subsubsection{Additional imaging methods}

As in human radiotherapy, additional imaging methods may be required to extract information for treatment planning for animals. From Single Photon Emission Computed Tomography (SPECT) or PET (Positron Emission Tomography) images information may be extracted to distinguish metabolically highly active regions by using the ${ }^{18} \mathrm{~F}$ deoxyglucose (FDG) tracer. The same imaging modality may be used to detect hypoxic (low in oxygen) regions in tumours with tracers such as ${ }^{18} \mathrm{~F}$-fluoromisonidazole (FMISO) or ${ }^{18} \mathrm{~F}$-fluoroazomycin-arabinoside (FAZA). Such information may serve to assess sub-regions of tumours that e.g. could be the subject of radiation boost studies. Also Magnetic Resonance (MR) imaging could be used in small animal treatment planning. Recently, a group from the University of Ghent, Belgium introduced an MRIbased workflow for irradiation of animal brain tumours [22]. This involves special MR sequences to generate pseudo CT images with a limited number of tissue classes. Ultrasound imaging can in principle also be used for visualization of soft tissue structures for small animal treatment planning, especially if the scanning procedure can be automated without the need for a human operator to manipulate the transducer. So far, to our knowledge this technique has not yet been reported in combination with precision irradiators. A few recent reports mentioned the use of specialized ultrasound techniques for the characterization of tissue damage after irradiation [23, 24]. For small animal studies there exist certain imaging techniques which are not 
Table 2.1: Overview of various treatment planning systems developed for small animal radio-

\begin{tabular}{|c|c|c|}
\hline TPS & company / institute & based on \\
\hline microRTP & $\begin{array}{l}\text { Washington University } \\
\text { USA }\end{array}$ & $\begin{array}{l}\text { Factorizaton of dose } \\
\text { distributions in water and } \\
\text { non-water geometries }\end{array}$ \\
\hline DOSCTP & $\begin{array}{l}\text { Princess Margaret Hospital } \\
\text { Toronto, USA }\end{array}$ & Monte Carlo simulation \\
\hline nameless & $\begin{array}{l}\text { Johns Hopkins University } \\
\text { Baltimore, USA } \\
\text { McGill University } \\
\text { Montreal, Canada }\end{array}$ & $\begin{array}{l}\text { Pinnacle (Philips) } \\
\text { Eindhoven, the Netherlands } \\
+ \text { Monte Carlo simulation }\end{array}$ \\
\hline RT_Image & $\begin{array}{l}\text { Stanford University } \\
\text { USA }\end{array}$ & Monte Carlo simulation \\
\hline nameless & $\begin{array}{l}\text { Dresden University } \\
\text { Germany }\end{array}$ & Monte Carlo simulation \\
\hline Muriplan & $\begin{array}{l}\text { XStrahl Ltd. } \\
\text { Camberley, UK } \\
\text { Johns Hopkins University } \\
\text { Baltimore, USA }\end{array}$ & superposition-convolution \\
\hline SmART-Plan & $\begin{array}{l}\text { Precision X-ray Inc. } \\
\text { North Branford, USA } \\
\text { Maastro Clinic } \\
\text { Maastricht, the Netherlands }\end{array}$ & Monte Carlo simulation \\
\hline
\end{tabular}

TPS, treatment planning system

useable in humans, such as optical [25] or photo-acoustic imaging [26], which may be useful to visualize certain processes in the tumour or healthy tissues. Phase contrast imaging [27] may be another imaging technique which could be integrated with small animal radiation research platforms. Multimodality imaging is expected to play a significant role in guiding treatment planning for preclinical studies or in assessing treatment outcome.

\subsection{Dose calculation models for small animals}

For accurate $\mathrm{kV}$ photon dose calculations, the most suitable technique is Monte Carlo simulation [10]. Less accurate techniques can be based e.g. on dose factorization, raytracing, superposition-convolution or other models. In what follows we will first discuss some of the simpler methods then followed by Monte Carlo simulation. An overview of TPS developed by various groups is given in table 2.1. 


\subsubsection{Analytical method for ${ }^{192}$ Ir irradiator}

One of the pioneering efforts in building a small animal precision irradiator originated from Washington University where a system was built based on irradiation with narrow beams with the ${ }^{192}$ Ir isotope. The system had a software interface for stage control, and a dedicated treatment planning system (microRTP) that was based on CERR, a MATLAB based research TPS [28]. The TPS was based on water geometries only. ${ }^{192} \mathrm{Ir}$ emits photons with energies from a few keV to $885 \mathrm{keV}$, therefore, the differences in absorbed dose in the different mouse tissues are not as large as for kV x-rays (figure 2.1), but their dose calculation accuracy could improve by taking non-water heterogeneities into account. The authors developed a fast analytical dose calculation scheme which consisted of a multiplication of various factors, some of which were derived from Monte Carlo simulations. This approach resembles the fast dose calculation methods that were available in older TPS once commonly employed in radiotherapy. A more accurate approach taking into account heterogeneities was considered [29], but it is not straightforward to adapt their parametrized model to include tissue heterogeneities. The fast algorithm was reported to result in dose errors limited to $10 \%$ in water, near beam edges and close to the entrance region for small fields $(<10 \mathrm{~mm}$ ). These researchers [30] reported a build-up region of less than $1.5 \mathrm{~mm}$ and their papers indicate that the beam penumbra extends up to $3 \mathrm{~mm}$. The latter is quite large compared to small structures in the animal that could be targeted in precision irradiation studies.

For animal $\mathrm{kV}$ irradiators a similar simple fast dose calculation model has been published [31]. Tissue heterogeneities were approximately taken into account by using estimates of radiological pathlengths of photons through tissues. These simplified approaches are inaccurate in non-water geometries, in particular in the kV energy range. These methods cannot provide estimates of photon spectra in tissues, which are needed to assess RBE. These methods can also not easily report the dose in the form of dose to medium-in-medium or dose to water-in-medium (see section 2.5.4) since they only provide dose to water-in-water. It was also not clear how these simple methods can provide absolute dose distributions; in their work only relative dose distributions were shown. It has been shown that the absolute output of small radiation beams is much harder to model accurately [32, 33]. 


\subsubsection{Superposition-convolution dose calculations}

A team from the Johns Hopkins University (Baltimore, USA) has developed a superposition-convolution algorithm for dose calculation in small animals, inspired by their previous work for radiotherapy MV photon beams [34, 35]. This method is based on the calculation of terma (total energy released to matter), which is obtained by multiplying the local photon energy fluence by the energy absorption coefficient. The local energy fluence is derived from the primary energy fluence emanating from the photon source, corrected for inverse square falloff with distance and photon attenuation. The method is essentially a ray-tracing approach, with inclusion of first order Compton scatter. An assumption in the model is that the dose deposition kernels are generated for different densities of water-like materials. Electron transport is included in the dose deposition kernel. For e.g. $225 \mathrm{keV}$ electrons (the maximum electron energy for the commercial $x$-ray irradiators) the electron range in most materials is limited to about $0.3 \mathrm{~mm}$, but in lung the range can be a few $\mathrm{mm}$, which can exceed several times the voxel dimensions. This method can use as input a photon spectrum or a phase space file (list of coordinates, energy, momentum etc. for a collection of particles collected at a certain position in space) obtained from a separate Monte Carlo simulation. Dose reporting is in terms of dose to water-in-medium, but can be converted to dose to medium-in-medium by multiplying the former by ratios of photon energy absorption coefficients of medium and water $[36,37]$, although this requires calculation of the local photon spectrum. It has been reported before that the superposition-convolution approach for kV photons may lead to problems in non-water media where dose discontinuities arise (e.g. bone, lung) [38]. The Johns Hopkins team recently described [39] the use of 3D Slicer $[40,41]$ to create a user interface for their TPS and their irradiator (SARRP, XStrahl Ltd, Camberley, UK). 3D Slicer is popular freeware for medical image visualization and analysis. Such an interface allows a broad range of users (not necessarily experts in treatment planning) to efficiently produce treatment plans for small animal studies. The Johns Hopkins team implemented the dose calculation on graphical processing units (GPU) in the Nvidia CUDA language for fast processing (sub-minute dose calculations). This dose calculation method is now commercially available as Muriplan (XStrahl Ltd).

\subsubsection{Monte Carlo simulation}

Monte Carlo simulation is the method of choice for accurate calculation of radiation dose and particle spectra in a voxelised animal, irradiated with $\mathrm{kV} x$-rays. This is be- 
cause of the dominance of the photo-electric effect, the presence of photon scatter and the ability to easily obtain photon spectra which can be used in radiation quality studies. The Monte Carlo technique models particle transport, interactions with other particles and electromagnetic fields, and production of secondary particles based on cross sections and transport theories. For applications in small animal treatment planning, Monte Carlo simulations can attain an accuracy of 1-2\%. An important issue in Monte Carlo transport is the cut-off at which to terminate the particle transport. For photons this is commonly set at $10 \mathrm{keV}$, whereas for the secondary electrons the cutoff has to be selected taking the voxel sizes into account, such that electrons do not erroneously contribute towards the dose in incorrect voxels. Particle transport cut-offs have been investigated in detail [42].

Recently, the Monte Carlo method has been used to model small animal radiation research platforms $[6,32,43]$. The in the past frequently heard complaint that Monte Carlo simulations are slow due to the large numbers of interactions to model, is nowadays less relevant with the availability of fast computers and parallel processing techniques. GPU computers are now also available to run simulations very efficiently [44] which will probably allow Monte Carlo simulations to play an important role in small animal treatment planning. A restriction could be in inverse planning [45] where a great number of dose calculations for individual beamlets need to be performed. It is imaginable that the beamlet dose calculations could be performed by a faster, less accurate method (e.g. a track length scoring Monte Carlo approach, or a faster analytical dose scoring technique) and that the final dose calculations after optimisation of the beamlets would be performed by a slower full Monte Carlo simulation.

A group in Toronto (Princess Margaret Hospital) developed DOSCTP [46], which is a graphical user interface enabling basic treatment planning of $\mathrm{kV}$ beams of $0.5-5$ $\mathrm{cm}$ diameter for small animals. The TPS initiates and calls the Monte Carlo code DOSXYZnrc [47] from within DOSCTP to execute dose calculations in a voxelised geometry. This basic TPS can be used to create 3D conformal plans. Figure 2.4 shows an example of an arc beam delivery in a mouse specimen. Another pioneering effort from the team at Johns Hopkins University, in collaboration with McGill University (Montreal, Canada) led to the development of a 3D kV Monte Carlo based TPS for their SARRP irradiator. They coupled the aforementioned Monte Carlo code DOSXYZnrc as a dose engine to a commercial human radiotherapy TPS (Pinnacle; Philips, Eindhoven, the Netherlands). The latter is used to create the beam geometry, the beam weights and the animal phantom. This information is then sent to the dose engine. Once the dose engine completed the calculations, the results are returned to 


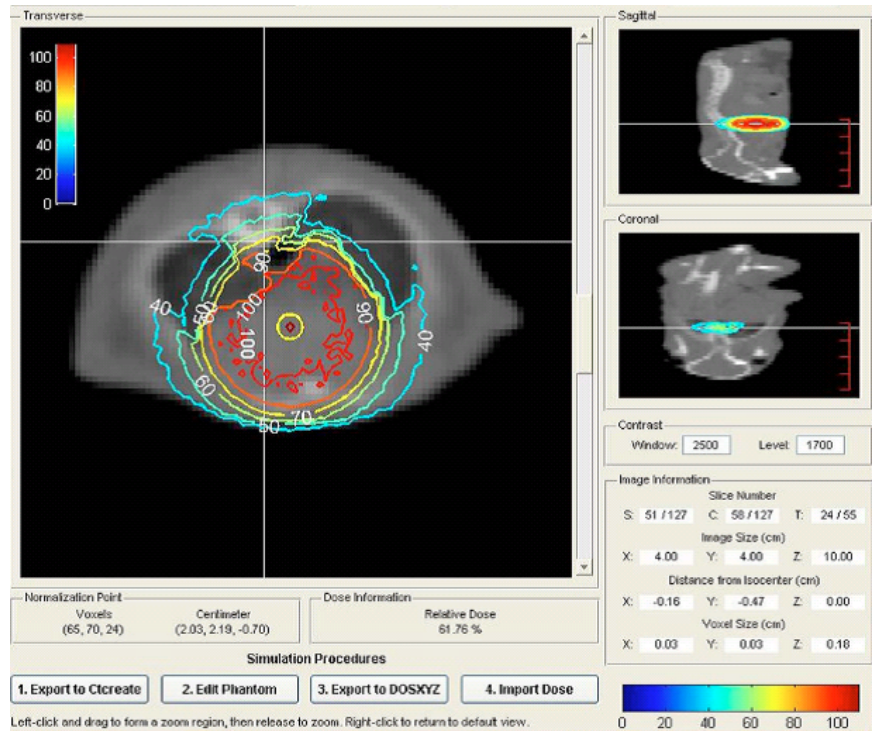

Figure 2.4: Monte Carlo calculated isodose distribution from an arc irradiation with $225 \mathrm{kVp}$ $x$-rays of a small target in a mouse chest, overlaid with a CBCT image. Reproduced from [46] with kind permission.

the TPS for dose display and analysis. The Pinnacle scripting environment is used to communicate with the Monte Carlo engine.

The combined TPS/dose engine uses a set of pre-computed phase space files for the SARRP nozzle collimators generated with BEAMnrc [43] Monte Carlo simulations. An early demonstration of their system was described in 2008 [7]. The Johns Hopkins group abandoned their efforts in Monte Carlo treatment planning in favour of the superposition-convolution method described in the previous section. Therefore, the abovementioned Monte Carlo based TPS is not commercially available.

More pioneering efforts came from a group at Stanford University where a microirradiator was developed by modifying a $120 \mathrm{kV}$ micro-CT scanner [48] (efforts now abandoned). They also developed an in-house TPS, RT Image, based on Monte Carlo simulations with the BEAMnrc/DOSXYZnrc codes. They were the first to extensively study the optimisation of dose calculation times for small animals (via so called variance reduction techniques) [42]. This group investigated the achievable dose conformality to small spherical and ellipsoidal targets in a phantom irradiated by discrete beams spread out in an arc [49]. They modelled centred and off-centred targets in a simple phantom. They showed that animal dose distributions are close approxi- 
mations to human lung tumour cases. These kinds of exercises are useful since in the new field of small animal treatment planning much experience still needs to be gained. These authors were also among the first to use dose volume histograms as a dose comparison tool for animal studies, which is a valuable tool for optimising and comparing dose plans.

Recently a group in Maastricht (the Netherlands) developed a standalone TPS SmART-Plan (Small Animal RadioTherapy planning system) [50]. The TPS is developed as a MATLAB program with a workflow that is similar as for a clinical TPS but simplified. Since a recent publication describes SmART-Plan in detail [50], we will only present a brief synopsis here. The workflow of SmART-Plan is very simple and robust; the tasks to be performed sequentially are (1) uploading the DICOM-compliant images of an animal of an imaging modality (e.g. CT), resampled on a different grid resolution and size if necessary; (2) image processing to assign densities and materials to each voxel in the CT to density and material (CT2MD) step, leading to a numerical phantom for Monte Carlo simulation; (3) structure definition (contouring) for tumour and healthy tissue delineation (structure contours are stored e.g. for later dose analysis); (4) definition of the static or arced radiation beams, resulting in a treatment plan and the input file for the simulations; (5) dose calculation with the DOSXYZnrc Monte Carlo dose engine, where parallel processing techniques are used to speed up the calculations; (6) dose analysis e.g. with dose volume histograms; (7) delivering a control file to the irradiator, which directs motion of the animal stage, positioning of the beams and irradiation of the specimen. SmART-Plan is commercially available from PXi (North Branford, USA).

The Maastricht group used the BEAMnrc Monte Carlo code to build one of the most detailed Monte Carlo models reported for the X-RAD 225Cx irradiator, including a sophisticated model for the electron focal spot on the x-ray target [33]. Monte Carlo dose calculations in animals can be performed in reasonable times nowadays, but simulation of an x-ray device still requires long calculations times, up to several tens of hours. This group also developed a faster analytical photon source model which allows speeding up the generation of an output phase space file, up to more than a thousand times faster.

SmART-Plan was validated by measurements with radiochromic film in various homogeneous and heterogeneous phantoms [50]. These were irradiated by three collimators under different irradiation angles and with two target isocentre locations each targeted with a dose of 5 Gy. For a heterogeneous solid water/solid bone/solid water slab phantom in the regions were the dose was between $80-100 \%$ of the maximum 

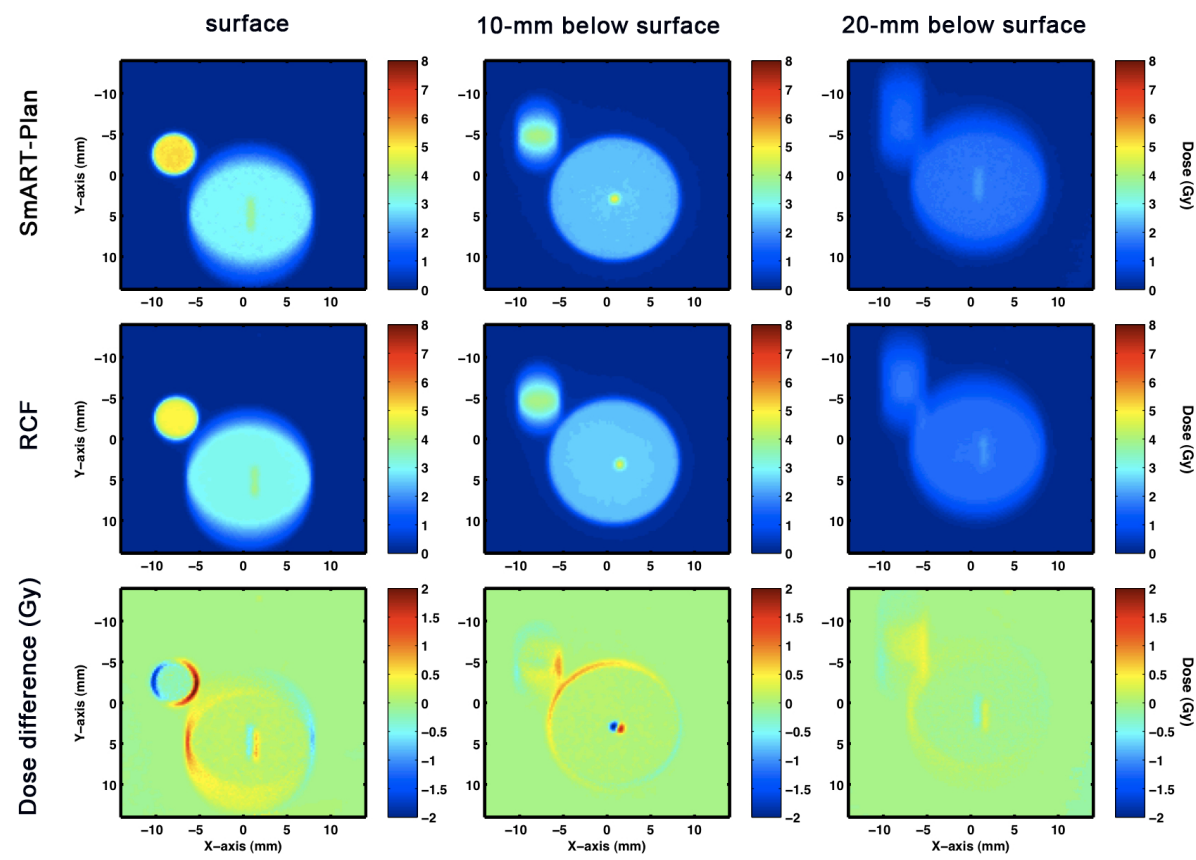

Figure 2.5: Comparison of SmART-Plan Monte Carlo calculated (top row) and radiochromic film measured (middle row) dose distributions at different depths in a solid water/solid bone/solid water slab phantom. Also shown are the dose differences (calculated - measured) in the bottom row. Dose differences are mostly limited to small regions in the beam penumbrae.

slice dose, about $1 \%$ of the voxels exhibited a dose difference of more than $10 \%$ of the maximum dose in that slice. Maximum absolute dose deviations of about 2 Gy were found but these were localized in small regions near the penumbra of the beams (figure 2.5). They noted that the $1 \mathrm{~mm}$ collimator was difficult to align precisely, which led to some dose discrepancies at $20 \mathrm{~mm}$ depth. They point out that the alignment of the smallest $1 \mathrm{~mm}$ collimator is critical for irradiation studies of small targets with these small beams.

Finally, we mention the effort to build a Monte Carlo simulation platform based on the Geant4 Monte Carlo code [51] for animal dose calculation from a consortium in Dresden [52]. They interface the Geant4 code with an in-house developed irradiator to perform contouring and setting up beams. As far as we know, no current animal TPS is capable of handling various types of irradiators, but this will undoubtedly change in the future. 


\subsubsection{Absolute dosimetry}

To deliver an accurate radiation dose to small animals the irradiator needs to be calibrated in terms of absolute dose, for which a calibration protocol such as AAPM Task Group 61 [53] needs to be followed. This is explained in more detail in e.g. [32, 54]. The dose calculation algorithm employed also needs to produce absolute dose distributions; this was covered in more detail in [32, 50].

\subsection{Issues related to treatment planning for small animals}

\subsubsection{Commissioning TPS}

An essential part in setting up a radiation research facility for small animals is the commissioning of the small animal irradiator and the associated TPS. This specialized subject may require particular equipment and measurement procedures for which we refer to the literature $[43,54,55]$ and the manufacturer's instructions.

\subsubsection{Specimen photon scatter}

Photon scatter for narrow beams of $\mathrm{kV}$ photon energies irradiating small animal geometries is a poorly studied subject. Photon scatter is important because it may degrade the CBCT imaging quality, and therefore compromise the conversion of the geometry into a voxel phantom. Scatter is also not or, at best, approximately modelled in most dose calculation algorithms for small animals, with the exception of Monte Carlo codes. Therefore, photon scatter may also degrade the dose calculation accuracy. In a recent study [56] it was shown that for a large field animal irradiator (160kVp; Faxitron CP-160, Tucson AZ) the surface dose to a small $2.8 \mathrm{~cm}$ diameter phantom could be overestimated by $15 \%$ when the conventional x-ray dosimetry protocol AAPM TG61 protocol [53] is used, compared to GATE [57] Monte Carlo simulations of the same setup. At the exit side of the phantom the dose overestimation amounted to more than $30 \%$. This study focused mostly on the lack of scatter for larger beams irradiating a very small phantom. For the narrow field precision irradiators no studies on photon scatter have been published yet, to our knowledge.

\subsubsection{Tissue segmentation for small animals}

In section 2.3.1 we outlined the procedure to assign soft tissue and bone media to voxel geometries, based on mass densities. For MV photons in most studies only four 
media are assigned for Monte Carlo simulations (air, lung, soft tissue, cortical bone), which appears to be sufficient. For kV irradiation of animals, the question arises how many tissue types are needed to attain a certain dose calculation accuracy. Recent work from Stanford University [58] demonstrated that the relative weight fraction of $\mathrm{Ca}$ and $\mathrm{P}$ correlates well with bone density in bone tissues; this allows easy segmentation of the bone type from the measured density. Another Stanford study [59] quantified the influence of tissue assignment on Monte Carlo dose calculations for different radiation sources (kV x-ray units: $120 \mathrm{kV}+2.5 \mathrm{~mm} \mathrm{Al,} 225 \mathrm{kV}+4 \mathrm{~mm} \mathrm{Al}, 225 \mathrm{kV}+0.5 \mathrm{~mm}$ $\mathrm{Cu}, 320 \mathrm{kV}+1.5 \mathrm{~mm} \mathrm{~Pb}+5 \mathrm{~mm} \mathrm{Sn}+1 \mathrm{~mm} \mathrm{Cu}+4 \mathrm{~mm} \mathrm{Al}$, and a brachytherapy ${ }^{192} \mathrm{Ir}$ source). The authors concluded there is no simple relationship between absorbed dose in tissue and mass density for photon energies $\leq 225 \mathrm{kV}$, indicating that mass density alone does not suffice to obtain accurate dose calculations. Dual energy CT images may aid in tissue segmentation, as discussed earlier [60-62].

They also compared a simple 4-tissue assignment scheme to an 8-tissue scheme and a 39-tissue scheme. Large dose errors were reported when the simpler tissue assignment schemes are used. The largest dose errors were obtained when adipose was inadvertently identified as muscle or cartilage. For a treatment plan of a mouse lung irradiation they also noticed that the target dose volume histogram (in the lung) was rather insensitive to tissue miss-assignment in the surrounding regions, whereas larger errors may occur in the organs at risk. This clearly shows the importance of correct tissue recognition, and of course all studies up to now assigned human tissues to animals, due to lack of information on animal tissue compositions.

\subsubsection{Dose reporting}

From previous sections it is clear that tissues need to be properly identified to ensure correct dose calculation in $\mathrm{kV}$ photon beams. An additional issue is dose reporting. The natural way for Monte Carlo simulations to score dose is in terms of dose to medium-in-medium $\left(D_{m, m}\right)$. Hereby photons are transported in all media, and when dose is scored in a voxel due to an interaction, the material interaction coefficients of the medium are used. However, dose may also be scored as dose to water-in-medium $\left(D_{w, m}\right)$. This is a quantity that is easier to measure, on which clinical practice is built, and most non-Monte Carlo algorithms report this quantity. To score $D_{w, m}$ photon transport is performed in the medium but the photon interaction coefficients of water are used when scoring the dose. $D_{m, m}$ and $D_{w, m}$ are two entirely different quantities, although they are both absorbed doses. For MV photons this issue also exists but it is of less significance with the difference limited to about $12 \%$ in cortical bone and much 
smaller in most other tissues. For $\mathrm{kV}$ photons the difference between the two dose quantities can be much larger (figure 2.1). Conversion of one of the dose quantities into the other involves knowledge of the ratios of energy absorption coefficients for low energy photons [37]. More complex conversions are also possible depending on the so-called cavity theory employed [63]. The conversion adds uncertainty to the dose reporting procedure. These two dose quantities exist, somewhat confusingly, next to each other [19,64, 65]. Which one correlates better with biological radiation effects is currently an open question and may eventually settle which dose quantity will be most frequently reported. In studies it should be clearly mentioned which dose reporting quantity is employed.

\subsubsection{Motion}

When a dose calculation in an animal is based on a relatively slowly acquired CBCT scan, motion of e.g. the lungs will smear out the organ volumes. When the aim is to precisely target certain structures, motion during the imaging and irradiation stages is an issue that needs to be addressed but which hasn't received much attention yet in the literature. This may require implementation of techniques such as time-resolved imaging and gated beam delivery, which are state of the art technologies known from human radiotherapy.

\subsection{Future developments}

Treatment planning for precision irradiation of small animals is not yet a mature field. Much effort is still needed to develop precise and accurate radiation planning software to achieve the full potential of the novel research platforms. These may then empower animal studies which may be translated successfully to human radiotherapy. TPS are only now becoming available that can handle the complexities of the combination of small beams, low energy photons and small geometric structures in animals. Future TPS will also be able to handle a rich variety of imaging modalities e.g. CT, PET, MRI, ultrasound, phase contrast $x$-ray, bioluminescence, fluorescence, photoacoustic imaging etc. Other beam modalities than low energy photons are emerging for precision-irradiation animal studies, such as low energy protons [66, 67]. Due to the complexities and uncertainties in low energy photon dose calculations there is also a need for beam delivery verification techniques e.g. based on onboard imagers [32]. 


\section{Chapter 2}

As the research platforms become more versatile, TPS will have to cope with techniques such as simultaneous motion of beam and stage, energy modulated beams, current modulated beams, gated beam delivery, motion-corrected beam delivery etc. To advance treatment planning for small animals many techniques can be borrowed from the mature field of treatment planning for human radiotherapy. An example is inverse treatment optimisation [45], whereby certain dose objectives and constraints are defined and then the most optimal plan is designed. Planning and radiation dose delivery to small animal models of unprecedented accuracy will be available in the near future, at a comparable level to human radiotherapy, enabling a wealth of studies. 


\section{References}

1. Popescu CC, Olivotto IA, Beckham WA, Ansbacher W, Zavgorodni S, Shaffer R, Wai ES, Otto K. Volumetric Modulated Arc Therapy Improves Dosimetry and Reduces Treatment Time Compared to Conventional Intensity-Modulated Radiotherapy for Locoregional Radiotherapy of LeftSided Breast Cancer and Internal Mammary Nodes. International Journal of Radiation Oncology*Biology*Physics 2010; 76: 287-295. doi: 10.1016/j.jijobp.2009.05.038.

2. Otto K. Volumetric modulated arc therapy: IMRT in a single gantry arc. Med Phys 2008; 35 : 310-317. doi: 10.1118/1.2818738.

3. Lin Z, Mechalakos J, Nehmeh S, Schoder H, Lee N, Humm J, Ling CC. The Influence of Changes in Tumor Hypoxia on Dose-Painting Treatment Plans Based on 18F-FMISO Positron Emission Tomography. International Journal of Radiation Oncology ${ }^{*}$ Biology ${ }^{*}$ Physics 2008; 70 : 1219-1228. doi: 10.1016/j.jijobp.2007.09.050.

4. Suit H, DeLaney T, Goldberg S, Paganetti H, Clasie B, Gerweck L, Niemierko A, Hall E, Flanz J, Hallman J, Trofimov A. Proton vs carbon ion beams in the definitive radiation treatment of cancer patients. Radiotherapy and Oncology 2010; 95: 3-22. doi: 10.1016/j.radonc.2010.01.015.

5. Grün R, Friedrich T, Elsässer T, Krämer M, Zink K, Karger CP, Durante M, Engenhart-Cabillic $\mathrm{R}$, Scholz M. Impact of enhancements in the local effect model (LEM) on the predicted RBEweighted target dose distribution in carbon ion therapy. Phys. Med. Biol. 2012; 57: 7261-7274. doi: 10.1088/0031-9155/57/22/7261.

6. Verhaegen F, Granton P, Tryggestad E. Small animal radiotherapy research platforms. Phys Med Biol 2011; 56: R55-83. doi: 10.1088/0031-9155/56/12/R01.

7. Wong J, Armour E, Kazanzides P, lordachita I, Tryggestad E, Deng H, Matinfar M, Kennedy C, Liu Z, Chan T, Gray O, Verhaegen F, McNutt T, Ford E, DeWeese TL. High-resolution, small animal radiation research platform with $\mathrm{x}$-ray tomographic guidance capabilities. Int. J. Radiat. Oncol. Biol. Phys. 2008; 71: 1591-1599. doi: 10.1016/j.jijrobp.2008.04.025.

8. Clarkson R, Lindsay PE, Ansell S, Wilson G, Jelveh S, Hill RP, Jaffray DA. Characterization of image quality and image-guidance performance of a preclinical microirradiator. Med Phys 2011; 38: 845-856. doi: 10.1118/1.3533947.

9. DesRosiers C, Mendonca MS, Tyree C, Moskvin V, Bank M, Massaro L, Bigsby RM, CaperallGrant A, Valluri S, Dynlacht JR, Timmerman R. Use of the Leksell Gamma Knife for localized small field lens irradiation in rodents. Technol. Cancer Res. Treat. 2003; 2: 449-454. doi: $10.1177 / 153303460300200510$.

10. Seco J, Verhaegen F. Monte Carlo Techniques in Radiation Therapy. 2013;

11. Bijl HP, van Luijk P, Coppes RP, Schippers JM, Konings AWT, van der Kogel AJ. Influence of adjacent low-dose fields on tolerance to high doses of protons in rat cervical spinal cord. International Journal of Radiation Oncology*Biology*Physics 2006; 64: 1204-1210. doi: 10.1016/j.jijobp.2005.06.046.

12. Debus J, Scholz M, Haberer T, Peschke P, Jäkel O, Karger CP, Wannenmacher M. Radiation Tolerance of the Rat Spinal Cord after Single and Split Doses of Photons and Carbon lons1. rare 2003; 160: 536-543. doi: 10.1667/3063.

13. Suplee C. XCOM: Photon Cross Sections Database. NIST 2009; Available from: https://www.nist.gov/pml/xcom-photon-cross-sections-database.

14. White DR, Booz J, Griffith RV, Spokas JJ, Wilson IJ. ICRU Report 44, Tissue Substitutes in Radiation Dosimetry and Measurement. J ICRU 1989; os23: NP-NP. doi: 10.1093/jicru/os23.1.Report44. 
15. Nikjoo H, Lindborg L. RBE of low energy electrons and photons. Phys. Med. Biol. 2010; 55 : R65-R109. doi: 10.1088/0031-9155/55/10/R01.

16. Granton PV, Dubois L, van Elmpt W, van Hoof SJ, Lieuwes NG, De Ruysscher D, Verhaegen F. A longitudinal evaluation of partial lung irradiation in mice by using a dedicated imageguided small animal irradiator. Int. J. Radiat. Oncol. Biol. Phys. 2014; 90: 696-704. doi: 10.1016/j.jijrobp.2014.07.004.

17. Hatton J, McCurdy B, Greer PB. Cone beam computerized tomography: the effect of calibration of the Hounsfield unit number to electron density on dose calculation accuracy for adaptive radiation therapy. Phys. Med. Biol. 2009; 54: N329. doi: 10.1088/0031-9155/54/15/N01.

18. Detombe SA, Dunmore-Buyze J, Petrov IE, Drangova M. X-ray dose delivered during a longitudinal micro-CT study has no adverse effect on cardiac and pulmonary tissue in C57BL/6 mice. Acta Radiol 2013; 54: 435-441. doi: 10.1177/0284185113475608.

19. Beaulieu L, Carlsson Tedgren A, Carrier J-F, Davis SD, Mourtada F, Rivard MJ, Thomson RM, Verhaegen F, Wareing TA, Williamson JF. Report of the Task Group 186 on model-based dose calculation methods in brachytherapy beyond the TG-43 formalism: current status and recommendations for clinical implementation. Med Phys 2012; 39: 6208-6236. doi: 10.1118/1.4747264.

20. Yang $\mathrm{C}-\mathrm{C}, \mathrm{Yu} \mathrm{J}-\mathrm{A}$, Yang B-H, Wu T-H. Optimization of the scan protocols for CT-based material extraction in small animal PET/CT studies. Nuclear Instruments and Methods in Physics Research Section A: Accelerators, Spectrometers, Detectors and Associated Equipment 2013; 731: 299-304. doi: 10.1016/j.nima.2013.03.007.

21. Boone JM, Velazquez O, Cherry SR. Small-animal X-ray dose from micro-CT. Mol Imaging 2004; 3: 149-158. doi: 10.1162/1535350042380326.

22. Gutierrez S, Descamps B, Vanhove C. MRI-Only Based Radiotherapy Treatment Planning for the Rat Brain on a Small Animal Radiation Research Platform (SARRP). PLoS One 2015; 10: doi: 10.1371/journal.pone.0143821. Available from: https://www.ncbi.nlm.nih.gov/pmc/articles/PMC4669183/.

23. Vlad RM, Kolios MC, Czarnota GJ. Ultrasound imaging of apoptosis: spectroscopic detection of DNA-damage effects at high and low frequencies. Methods Mol. Biol. 2011; 682: 165-187. doi: 10.1007/978-1-60327-409-8_13.

24. Vlad RM, Brand S, Giles A, Kolios MC, Czarnota GJ. Quantitative Ultrasound Characterization of Responses to Radiotherapy in Cancer Mouse Models. Clin Cancer Res 2009; 15: 2067-2075. doi: 10.1158/1078-0432.CCR-08-1970.

25. Luker GD, Luker KE. Optical imaging: current applications and future directions. J. Nucl. Med. 2008; 49: 1-4. doi: 10.2967/jnumed.107.045799.

26. Lungu GF, Li M-L, Xie X, Wang LV, Stoica G. In vivo imaging and characterization of hypoxiainduced neovascularization and tumor invasion. Int. J. Oncol. 2007; 30: 45-54.

27. Bravin A, Coan P, Suortti P. X-ray phase-contrast imaging: from pre-clinical applications towards clinics. Phys. Med. Biol. 2012; 58: R1-R35. doi: 10.1088/0031-9155/58/1/R1.

28. Deasy JO, Blanco AI, Clark VH. CERR: a computational environment for radiotherapy research. Med Phys 2003; 30: 979-985. doi: 10.1118/1.1568978.

29. Stojadinovic S, Low DA, Hope AJ, Vicic M, Deasy JO, Cui J, Khullar D, Parikh PJ, Malinowski KT, Izaguirre EW, Mutic S, Grigsby PW. MicroRT-small animal conformal irradiator. Med Phys 2007; 34: 4706-4716. doi: 10.1118/1.2799887.

30. Stojadinovic S, Low DA, Vicic M, Mutic S, Deasy JO, Hope AJ, Parikh PJ, Grigsby PW. Progress toward a microradiation therapy small animal conformal irradiator. Med Phys 2006; 
33: 3834-3845. doi: 10.1118/1.2349693.

31. Marco-Rius I, Wack L, Tsiamas P, Tryggestad E, Berbeco R, Hesser J, Zygmanski P. A fast analytic dose calculation method for arc treatments for kilovoltage small animal irradiators. Physica Medica 2013; 29: 426-435. doi: 10.1016/j.ejmp.2013.02.003.

32. Granton PV, Podesta M, Landry G, Nijsten S, Bootsma G, Verhaegen F. A combined dose calculation and verification method for a small animal precision irradiator based on onboard imaging. Med Phys 2012; 39: 4155-4166. doi: 10.1118/1.4725710.

33. Granton PV, Verhaegen F. On the use of an analytic source model for dose calculations in precision image-guided small animal radiotherapy. Phys. Med. Biol. 2013; 58: 3377. doi: 10.1088/0031-9155/58/10/3377.

34. Jacques R, Wong J, Taylor R, McNutt T. Real-time dose computation: GPU-accelerated source modeling and superposition/convolution. Med Phys 2011; 38: 294-305. doi: 10.1118/1.3483785.

35. Jacques R, Taylor R, Wong J, McNutt T. Towards real-time radiation therapy: GPU accelerated superposition/convolution. Comput Methods Programs Biomed 2010; 98: 285-292. doi: 10.1016/j.cmpb.2009.07.004

36. Siebers JV, Keall PJ, Nahum AE, Mohan R. Converting absorbed dose to medium to absorbed dose to water for Monte Carlo based photon beam dose calculations. Phys. Med. Biol. 2000; 45: 983-995. doi: 10.1088/0031-9155/45/4/313.

37. Landry G, Reniers B, Pignol J-P, Beaulieu L, Verhaegen F. The difference of scoring dose to water or tissues in Monte Carlo dose calculations for low energy brachytherapy photon sources. Medical Physics 2011; 38: 1526-1533. doi: 10.1118/1.3549760.

38. Verhaegen F. Heterogeneity corrected convolution dose calculation for $\mathrm{kV} x$-rays versus Monte Carlo simulation. 2004;

39. Cho N, Kazanzides P. A Treatment Planning System for the Small Animal Radiation Research Platform (SARRP) based on 3D Slicer. The MIDAS Journal 2012; 866.

40. Pinter C, Lasso A, Wang A, Jaffray D, Fichtinger G. SlicerRT: radiation therapy research toolkit for 3D Slicer. Med Phys 2012; 39: 6332-6338. doi: 10.1118/1.4754659.

41. Pieper S, Halle M, Kikinis R. 3D Slicer. 2004 2nd IEEE International Symposium on Biomedical Imaging: Nano to Macro (IEEE Cat No. 04EX821) 2004; 632-635 Vol. 1. doi: 10.1109/ISBI.2004.1398617.

42. Bazalova M, Zhou H, Keall PJ, Graves EE. Kilovoltage beam Monte Carlo dose calculations in submillimeter voxels for small animal radiotherapy. Med Phys 2009; 36: 4991-4999. doi: 10.1118/1.3238465.

43. Tryggestad E, Armour M, lordachita I, Verhaegen F, Wong JW. A comprehensive system for dosimetric commissioning and Monte Carlo validation for the small animal radiation research platform. Phys Med Biol 2009; 54: 5341-5357. doi: 10.1088/0031-9155/54/17/017.

44. Hissoiny S, Ozell B, Bouchard H, Després P. GPUMCD: A new GPU-oriented Monte Carlo dose calculation platform. Medical Physics 2011; 38: 754-764. doi: 10.1118/1.3539725.

45. Stewart JMP, Lindsay PE, Jaffray DA. Two-dimensional inverse planning and delivery with a preclinical image guided microirradiator. Med Phys 2013; 40: 101709. doi: 10.1118/1.4819935.

46. Chow JCL, Leung MKK. Treatment planning for a small animal using Monte Carlo simulation. Med Phys 2007; 34: 4810-4817. doi: 10.1118/1.2805254.

47. Walters B, Kawrakow I, Rogers DWO. DOSXYZnrc Users Manual. 2005; 132. Available from: https://nrc-cnrc.github.io/EGSnrc/doc/pirs794-dosxyznrc.pdf. 
48. Graves EE, Zhou H, Chatterjee R, Keall PJ, Gambhir SS, Contag CH, Boyer AL. Design and evaluation of a variable aperture collimator for conformal radiotherapy of small animals using a microCT scanner. Med Phys 2007; 34: 4359-4367. doi: 10.1118/1.2789498.

49. Motomura AR, Bazalova M, Zhou H, Keall PJ, Graves EE. Investigation of the effects of treatment planning variables in small animal radiotherapy dose distributions. Medical Physics 2010; 37: 590-599. doi: 10.1118/1.3276738.

50. van Hoof SJ, Granton PV, Verhaegen F. Development and validation of a treatment planning system for small animal radiotherapy: SmART-Plan. Radiother Oncol 2013; 109: 361-366. doi: 10.1016/j.radonc.2013.10.003.

51. Agostinelli S et al. Geant4-a simulation toolkit. Nuclear Instruments and Methods in Physics Research Section A: Accelerators, Spectrometers, Detectors and Associated Equipment 2003; 506: 250-303. doi: 10.1016/S0168-9002(03)01368-8.

52. Tillner F, Thute P, Hietschold V, Khaless A, Lukas M, Rimarzig B. Bildgeführtes Präzisionsbestrahlungsgerät für Kleintiere (SAIGRT) - vom Konzept zur praktischen Umsetzung. Abstractband DGMP 2012; 43: 647-650.

53. Ma CM, Coffey CW, DeWerd LA, Liu C, Nath R, Seltzer SM, Seuntjens JP, American Association of Physicists in Medicine. AAPM protocol for 40-300 kV x-ray beam dosimetry in radiotherapy and radiobiology. Med Phys 2001; 28: 868-893. doi: 10.1118/1.1374247.

54. Lindsay PE, Granton PV, Gasparini A, Jelveh S, Clarkson R, van Hoof S, Hermans J, Kaas J, Wittkamper F, Sonke J-J, Verhaegen F, Jaffray DA. Multi-institutional dosimetric and geometric commissioning of image-guided small animal irradiators. Med Phys 2014; 41: 031714. doi: 10.1118/1.4866215.

55. Wack L, Ngwa W, Tryggestad E, Tsiamas P, Berbeco R, Ng SK, Hesser J, Zygmanski P. High throughput film dosimetry in homogeneous and heterogeneous media for a small animal irradiator. Physica Medica 2014; 30: 36-46. doi: 10.1016/j.ejmp.2013.02.002.

56. Noblet C, Chiavassa S, Paris F, Supiot S, Lisbona A, Delpon G. Underestimation of dose delivery in preclinical irradiation due to scattering conditions. Physica Medica 2014; 30: 63-68. doi: 10.1016/j.ejmp.2013.03.001.

57. Jan S, Benoit D, Becheva E, Carlier T, Cassol F, Descourt P, Frisson T, Grevillot L, Guigues L, Maigne L, Morel C, Perrot Y, Rehfeld N, Sarrut D, Schaart DR, Stute S, Pietrzyk U, Visvikis D, Zahra N, Buvat I. GATE V6: a major enhancement of the GATE simulation platform enabling modelling of CT and radiotherapy. Phys. Med. Biol. 2011; 56: 881-901. doi: 10.1088/00319155/56/4/001.

58. Zhou H, Keall PJ, Graves EE. A bone composition model for Monte Carlo x-ray transport simulations. Medical Physics 2009; 36: 1008-1018. doi: 10.1118/1.3077129.

59. Bazalova M, Graves EE. The importance of tissue segmentation for dose calculations for kilovoltage radiation therapy. Medical Physics 2011; 38: 3039-3049. doi: 10.1118/1.3589138.

60. Landry G, Granton PV, Reniers B, Ollers MC, Beaulieu L, Wildberger JE, Verhaegen F. Simulation study on potential accuracy gains from dual energy CT tissue segmentation for low-energy brachytherapy Monte Carlo dose calculations. Phys Med Biol 2011; 56: 6257-6278. doi: 10.1088/0031-9155/56/19/007.

61. Landry G, Reniers B, Granton PV, van Rooijen B, Beaulieu L, Wildberger JE, Verhaegen F. Extracting atomic numbers and electron densities from a dual source dual energy CT scanner: experiments and a simulation model. Radiother Oncol 2011; 100: 375-379. doi: 10.1016/j.radonc.2011.08.029.

62. Landry G, Parodi K, Wildberger JE, Verhaegen F. Deriving concentrations of oxygen and carbon 
in human tissues using single- and dual-energy CT for ion therapy applications. Phys. Med. Biol. 2013; 58: 5029-5048. doi: 10.1088/0031-9155/58/15/5029.

63. Tedgren AC, Carlsson GA. Specification of absorbed dose to water using model-based dose calculation algorithms for treatment planning in brachytherapy. Phys Med Biol 2013; 58: 2561-2579. doi: 10.1088/0031-9155/58/8/2561.

64. Thomson RM, Tedgren ÅCarlsson, Williamson JF. On the biological basis for competing macroscopic dose descriptors for kilovoltage dosimetry: cellular dosimetry for brachytherapy and diagnostic radiology. Phys. Med. Biol. 2013; 58: 1123-1150. doi: 10.1088/0031-9155/58/4/1123.

65. Enger SA, Ahnesjö A, Verhaegen F, Beaulieu L. Dose to tissue medium or water cavities as surrogate for the dose to cell nuclei at brachytherapy photon energies. Phys. Med. Biol. 2012; 57: 4489-4500. doi: 10.1088/0031-9155/57/14/4489.

66. Narayanan M. Monte Carlo Simulation of a Precision Proton Radiotherapy Platform Designed for Small-Animal Experiments. 2013;

67. Sandison GA, Ford E, Stewart RD, Narayanan M, Hug E, Laramore GE. A small animal proton IGRT facility for comparative studies of tumor and normal tissue responses. 2013; 

Development and validation of a treatment planning system for small animal radiotherapy: SmART-Plan

authors

Stefan J van Hoof, Patrick V Granton, Frank Verhaegen

adapted from

Radiotherapy \& Oncology 2013; 109: 361-366 


\section{Abstract}

\section{Background and purpose}

Image guided equipment for precision irradiation of small animals for preclinical radiotherapy research became recently available. To enable downscaled radiotherapy studies that can be translated into human radiotherapy knowledge, a treatment planning system for preclinical studies is required.

\section{Material and methods}

A dedicated treatment planning system (SmART-Plan) for small animal radiotherapy studies was developed. It is based on Monte Carlo simulation of particle transport in an animal. The voxel geometry is derived from the onboard cone beam CT imaging panel. SmARTPlan was validated using radiochromic film (RCF) dosimetry in various phantoms: uniform, multislab and a realistic plasticized mouse geometry.

\section{Results}

Good agreement was obtained between SmART-Plan dose calculations and RCF dose measurements in all phantoms. For various delivered plans agreement was obtained within $10 \%$ for the majority of the targeted dose region, with larger differences between $10 \%$ and $20 \%$ near the penumbra regions and for the smallest $1 \mathrm{~mm}$ collimator. Absolute depth and lateral dose distributions showed better agreement for 5 and $15-\mathrm{mm}$ collimators than for a $1 \mathrm{~mm}$ collimator, indicating that accurate dose prediction for the smallest field sizes is difficult.

\section{Conclusion}

SmART-Plan offers a useful dose calculation tool for preclinical small animal irradiation studies 


\subsection{Introduction}

There is a limited amount of information that can be gleaned from clinical trials with radiotherapy patients and it has been hypothesized that greater insight can be obtained from downscaling radiotherapy to the scale of small animals such as mice and rats [1]. This is because of the rapid development of realistic preclinical orthotopic tumour models in animals and because many radiobiological experiments in the past were conducted using radiation conditions that are not representative for the current radiotherapy practice. Most past animal experiments used fairly large radiation beams, did not have image guidance and had imprecise or no treatment planning. Hence the recent development of dedicated image guided precision irradiation research platforms (see [2] and references therein) and other bespoke devices based on modified micro-CT devices [3] for use in preclinical studies.

The latest commercial small animal radiation research platforms consist of a heavyduty x-ray machine (typically $225 \mathrm{kV}$ ), precision photon beam collimators (down to $1 \mathrm{~mm}$ field size), a 3D moving animal stage and an x-ray imaging panel for image guidance. These devices are computer controlled and are capable of delivering precise static or dynamic arc radiation beams to small targets, with significant sparing of healthy tissue compared to older experiments on which our radiobiology knowledge is built.

While these devices constitute a major technological advance, another element is needed to ensure that complex radiation dose distributions can be delivered to small animals. A dedicated treatment planning system (TPS) that can plan beam delivery to arbitrarily shaped targets with complex beam arrangements is needed to downscale human radiotherapy to the small animal level. Current clinical TPS can usually not handle dose calculations for kV x-rays, very small beams, and are often not equipped to deal with multimodality images with very small voxels. Hence the need for a TPS which may handle these aspects and can also be used for high-throughput animal experiments for example in drug-radiation synergy studies. This work describes the ongoing development and validation of a dedicated small animal TPS, SmART-Plan (where SmART stands for Small Animal RadioTherapy).

There are significant differences between treatment planning for small animals and human patients. Chief among them is the use of very small beams of kV $x$-ray photons. In the commercially available devices a broad $x$-ray spectrum of about $225 \mathrm{kV}$ is used for radiotherapy studies with collimators of a fixed beam size of between $1 \mathrm{~mm}$ and several centimetres. The x-ray tube is mounted on a movable support to enable 


\section{Chapter 3}

irradiation of the specimen from different sides. The same x-ray tube, but with a lower $\mathrm{kV}$ setting is used to create a cone beam CT (CBCT) image of the specimen. The imaging panel typically has a high resolution of about $100 \mu \mathrm{m}$, resulting in 3D CBCT images with a large number of voxels easily ranging in the tens of millions, imposing demands on computer memory.

For $\mathrm{kV}$ photons the interaction coefficients in tissues depend on both composition and density. Therefore, whereas for radiotherapy patient dose calculations it usually suffices to consider the geometry to consist solely of water with a density derived from the CT images, for $\mathrm{kV} x$-ray dose calculations in the 40-225 kV photon energy range, knowledge of the $x$-ray spectrum and the tissue composition is required. This is discussed in more detail in the supplementary material. Currently, animal dose calculations use human tissue compositions due to a lack of available data on animal tissue compositions.

Another issue related to small animal dose calculation is the use of very small fields $(1 \mathrm{~mm}-$ few $\mathrm{cm})$. The output of an x-ray device drops significantly for small collimator sizes expressed as radiation dose rate per unit $\mathrm{x}$-ray tube current. This is due to a lack of scatter equilibrium in tissue for the smallest fields but also due to obscuring of the electron focal spot on the x-ray target [4]. 


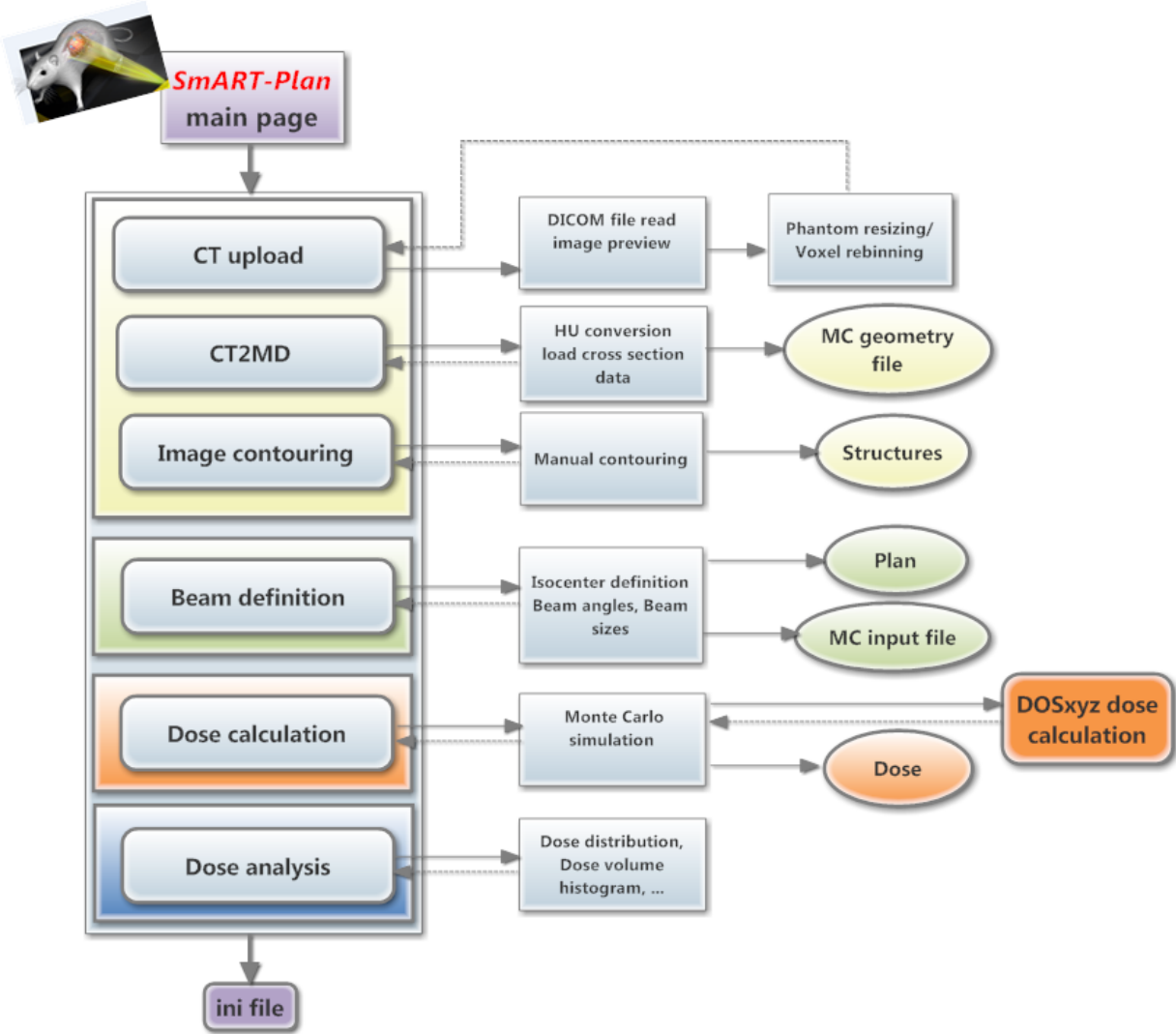

Figure 3.1: Flowchart of SmART-Plan. The tasks outlined on the left are to be performed sequentially from the top to the bottom.

\subsection{Materials and methods}

\subsubsection{Development of treatment planning platform}

SmART-Plan was developed in MATLAB (v7.11.0.584, R2010b, The MathWorks Inc., Natrick, Ma) and is distributed as a compiled executable program on a Linux-based platform (Ubuntu 12.04 or later). Figure 3.1 shows the design of SmART-Plan. It performs absolute dose calculations on user-generated voxel phantoms from reconstructed CBCT images by interfacing with the Monte Carlo (MC) program DOSXYZnrC (v1.47, 2011, NRCC, Canada). MC simulations of photon and electron transport are performed. The dose calculations are performed on a parallel computing architecture with 24 processors (12 core $2.67 \mathrm{GHz}$ Intel Xeon with 24 threads total). The x-ray device and its collimator are modelled by a photon source (i.e. phase space files) 
from a previous $\mathrm{MC}$ simulation [5]. Currently only the most common treatment photon spectrum is modelled (225 kVp, $0.32 \mathrm{~mm}$ Cu filtration). SmART-Plan is validated in this paper for a specific research platform (X-Rad 225Cx; Precision X-ray Inc., North Branford, CT), but should be equally usable with similar systems.

\subsubsection{CT image reconstruction}

CBCT scanning was performed using the research platform and the accompanying PilotCal software (v1.8, Precision X-ray Inc. North Branford, USA) at $80 \mathrm{kVp}$, typical for small animal micro-CT platforms to produce a sufficient signal at the detector without excess imaging dose to the animal [6]. 3D images (210 $\mu \mathrm{m}$ sided voxels) were reconstructed by back projection. A Hounsfield Unit $(\mathrm{HU})$ to density calibration of the CBCT scanner was performed with a phantom with known tissue substitute inserts (Model 467, Gammex RMI, Middleton, WI).

\subsubsection{Workflow of treatment planning platform}

The flowchart of the SmART-Plan process is shown in figure 3.1. Dose distributions and the final treatment plan are based on a CBCT image acquired with the animal in the treatment position. The CBCT image is imported in the CT upload module and can be cropped and resized to speed up MC dose calculations. Assignment of materials and densities is an essential part of the process not found in a human TPS and the CT2MD (CT to Material and Density) module is dedicated to this step. Any number of materials can be assigned based on thresholds in the HU-density histogram; densities are assigned based on the HU to density calibration curve. Required material photon interaction data are read from a database of human tissues, since the atomic composition of animal tissue is poorly known. A voxel phantom is created that serves as input for the MC dose calculation. Optionally, structure contours can be created to aid treatment plan evaluation. The planning of isocentres and static/dynamic beams is performed in the beam definition module. An estimated number of histories to be simulated is calculated based on the desired statistical uncertainty. A default selection of transport parameters is provided for the user based on previously published work of $\mathrm{kV}$ dose calculations [5, 7] but can be edited by the user. The dose calculation module takes care of the complete interface with the MC dose engine and dose distributions are imported and displayed automatically. Thereafter, dose distributions and dose volume histograms can be evaluated and optimized by adjusting beam weights. 
Finally, an ini file is generated which serves as input for the PilotCal software to execute the irradiation. See the supplementary material for screenshots of examples of some SmART-Plan modules.

\subsubsection{Validation of treatment planning platform}

Absolute dose reporting in SmART-Plan was performed by deriving a calibration factor from a comparison of a MC dose calculation in a water phantom and an absolute dose measurement using an ionization chamber with a reference field of $30 \times 40 \mathrm{~cm}$ at source to surface distance of $29.9 \mathrm{~cm}$. By modelling the exact geometry as used in the experiment the conversion factor from dose per particle (MC) to dose per mAs (experiment) can be determined. This conversion factor is then valid for all simulations with the same photon spectrum [5]. The measurements determined the dose to the surface of the water phantom by following a medium energy $\mathrm{x}$-ray dosimetry protocol [8]. Validation of SmART-Plan was performed by examining calculated absolute depth dose curves and dose distributions from multiple-target irradiation plans in uniform and heterogeneous phantoms, compared against radiochromic film (RCF) (GafChromic EBT2, Ashland Specialty Ingredients, Wayne, NJ) measurements for 1, 5 , and $15 \mathrm{~mm}$ circular collimators. RCF was used throughout this study for dose verification due to its superior spatial resolution, near water-equivalence for dosimetry, and the avoidance of a pre-scan when using the triple-channel read out procedure [9]. The RCF was calibrated with the research platform for a range of doses. Films were read out ( $225 \mathrm{dpi}$ in 48 bit, 16 bit per colour channel) 24 hours after exposure with a flatbed scanner Epson PRO V750 (Seiko Epson Corporation, Japan) [9]. To validate absolute depth dose distributions for the three collimators, first a CBCT image was acquired of a solid water slab phantom. A target dose of 5 Gy was prescribed to the surface of the phantom and MC dose calculations were launched to reach a statistical uncertainty of less than $2 \%$. Next, the ini file (figure 3.1) was generated that includes the beam-on time and the target isocentre location that the positioning stage moves toward prior to irradiation. Depth dose measurements for the collimators were then performed and repeated three times by exposing five RCF pieces on top and at different depths in the phantom. Measured and calculated depth dose distributions were compared by taking the average dose in a region from the maximum dose value to $80 \%$ thereof in both datasets. More treatment plans were created for three other phantoms. The first two slab phantoms consisted of three RCF pieces sandwiched on top and between three pieces of $10 \mathrm{~mm}$ thick solid water $(5 \times 8 \mathrm{~cm} 2)$ slabs, with the second phantom differing only by replacing the middle solid water slab by bone sub- 
stitute (SB3, Gammex-RMI, Middleton, WI). Treatment plans for these two phantoms made use of the three collimators under different irradiation angles and with two target isocentre locations each targeted with a dose of 5 Gy. Finally, a treatment plan was applied to a plasticized heterogeneous mouse specimen (PlastiMouse) developed by our anatomy department. The PlastiMouse phantom was sliced lengthwise to allow insertion of RCF. 3 Gy was prescribed from a static $5 \mathrm{~mm}$ collimator beam targeted from above.
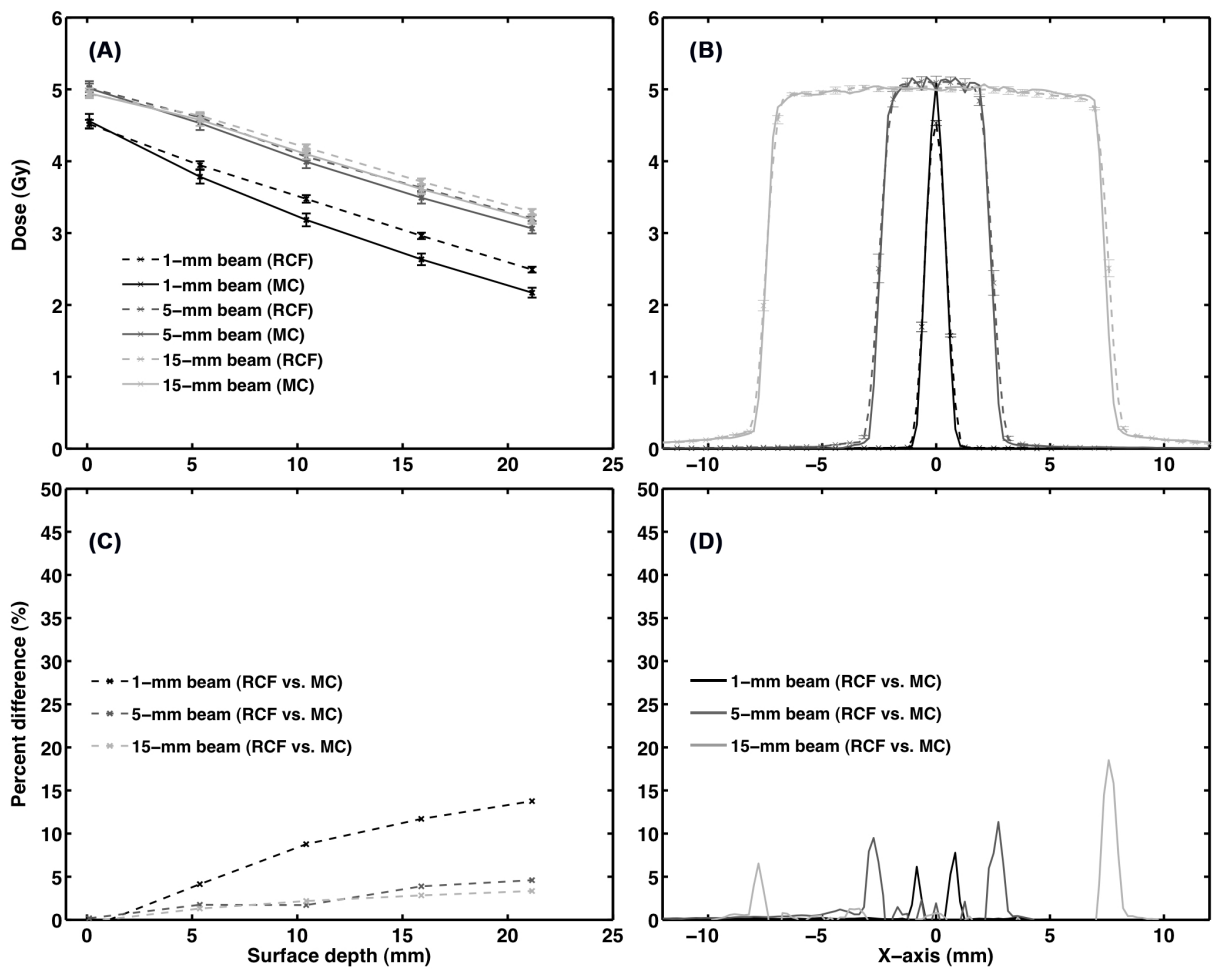

Figure 3.2: Measured (RCF) and computed (MC, SmART-Plan) absolute depth dose (A) and lateral dose profiles, parallel to the cathode/anode direction (B). Panels (C) and (D) display local differences (measured - calculated) dose. 
Table 3.1: Measured RCF and calculated SmART-Plan OF for three circular collimators and their uncertainty.

\begin{tabular}{llll}
\hline field $\varnothing(\mathrm{mm})$ & OF RCF $(-)$ & OF SmART-Plan $(-)$ & correction factor $(-)$ \\
\hline 1 & $0.53 \pm 0.01$ & $0.67 \pm 0.02$ & $0.79 \pm 0.05$ \\
5 & $0.85 \pm 0.02$ & $0.86 \pm 0.02$ & - \\
15 & $0.90 \pm 0.01$ & $0.90 \pm 0.03$ & - \\
$30 \times 40$ ref & $1.00 \pm 0.01$ & $1.00 \pm 0.02$ & - \\
\hline \multicolumn{2}{l}{ OF, output factor } \\
\multicolumn{2}{l}{ RCF, radiochromic film }
\end{tabular}

\subsection{Results}

\subsubsection{Validation of the treatment planning system}

Following the TG61 guidelines the dose rate at the surface of a solid water phantom was found to be $0.246 \mathrm{~Gy} / \mathrm{min} / \mathrm{mA}$ [8]. Table 3.1 compares measured and calculated output factors (OF) at the surface; for the smallest beam an output correction factor is required. Measured and calculated absolute dose distributions and lateral dose profiles (figure 3.2) showed good agreement within $5 \%$ for the 5 and $15 \mathrm{~mm}$ collimator at all depths. For the smallest $1 \mathrm{~mm}$ collimator a maximum difference of $13 \%$ at $21 \mathrm{~mm}$ depth was obtained. Lateral dose profiles showed good agreement except in the penumbra regions. Dose calculation times for each collimator were: 76,124 , and 495 seconds for the 1, 5, and $15 \mathrm{~mm}$ collimator, respectively, reflecting the larger number of particles required to simulate larger beams to achieve the same statistical accuracy. The multiple beam/multiple target treatment plan delivered in the two slab phantom geometries achieved good agreement between the RCF measurements and the SmART-Plan dose calculations, showing maximum absolute deviations of about 2 Gy localized near the penumbra of the beams (figure 3.3, 3.8). For the uniform solid water phantom in the regions with doses between $80 \%$ and $100 \%$ of the maximum dose in a slice, only $1 \%$ of the voxels exhibited a dose difference of more than $10 \%$ with respect to the maximum dose in that slice. For the heterogeneous slab phantom similar differences were reported except for the film piece at $20 \mathrm{~mm}$ below the surface where larger discrepancies were noted. The primary cause for this discrepancy is a misalignment in the targeting of the smallest beam $(1 \mathrm{~mm})$. When voxels with doses between $20 \%$ and $100 \%$ of the maximum dose are considered, only $4 \%$ of the voxels show a discrepancy exceeding $10 \%$. The total dose calculation time in SmARTPlan for both phantoms was about 550 seconds. SmART-Plan launches all the parallel dose calculations simultaneously and is therefore only limited by the job with the 
longest calculation time. Figure 3.4 demonstrates that for a realistic mouse phantom (PlastiMouse) the agreement between calculated and measured dose is good, with significant dose differences limited to the small beam penumbra region for a $5 \mathrm{~mm}$ collimator. For voxels with doses between $80 \%$ and $100 \%$ of the maximum dose in the slice containing the RCF, all voxel doses were within $10 \%$ of the maximum dose in that slice.
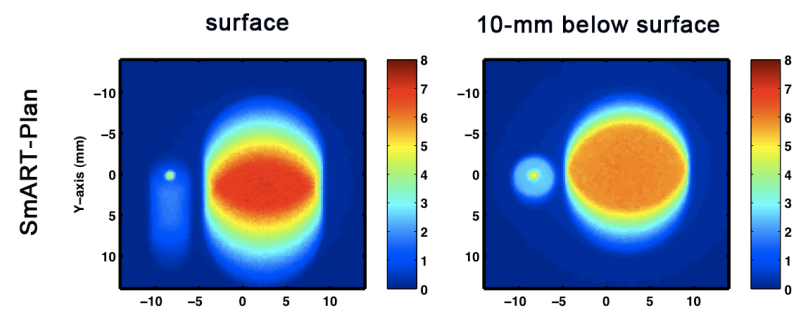

20-mm below surface
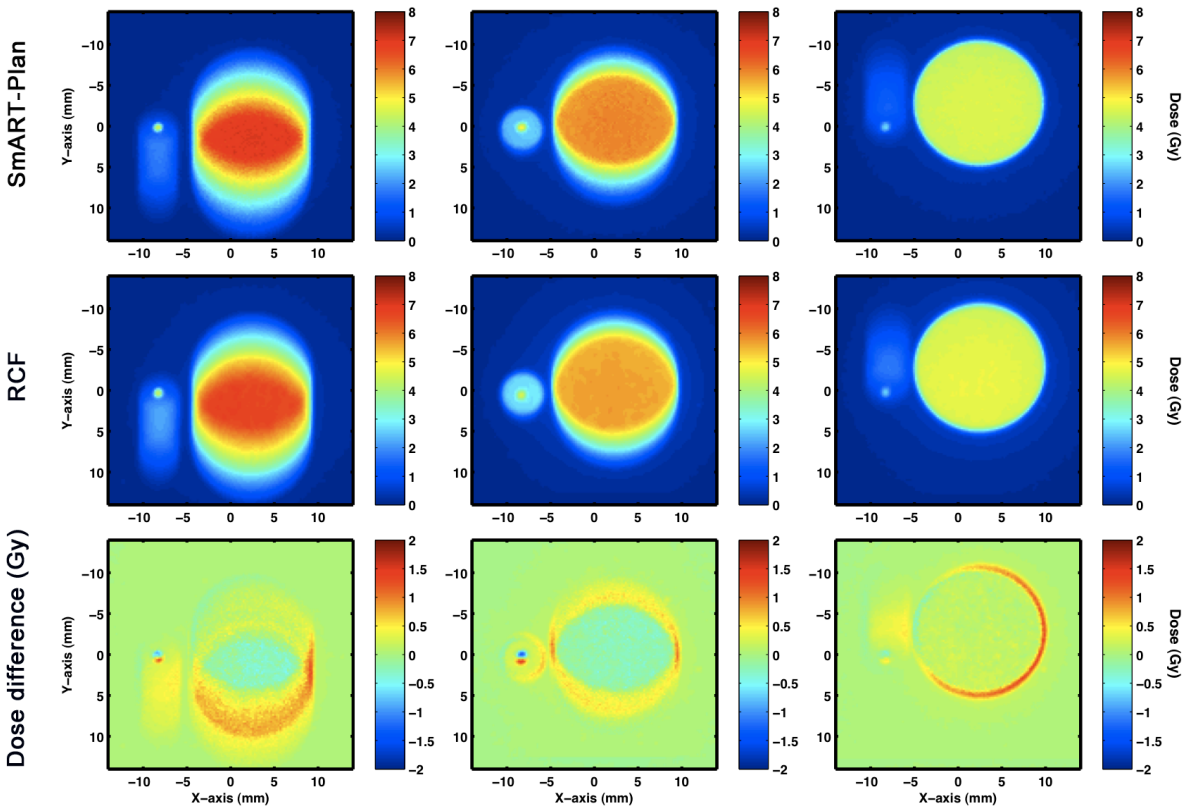

Figure 3.3: Comparison of (top row) calculated and (middle row) RCF measured dose distributions in a uniform solid water phantom. Also shown are the dose differences (calculated measured) in the bottom row. At various depths the 1,5 and $15 \mathrm{~mm}$ beams can be observed. 


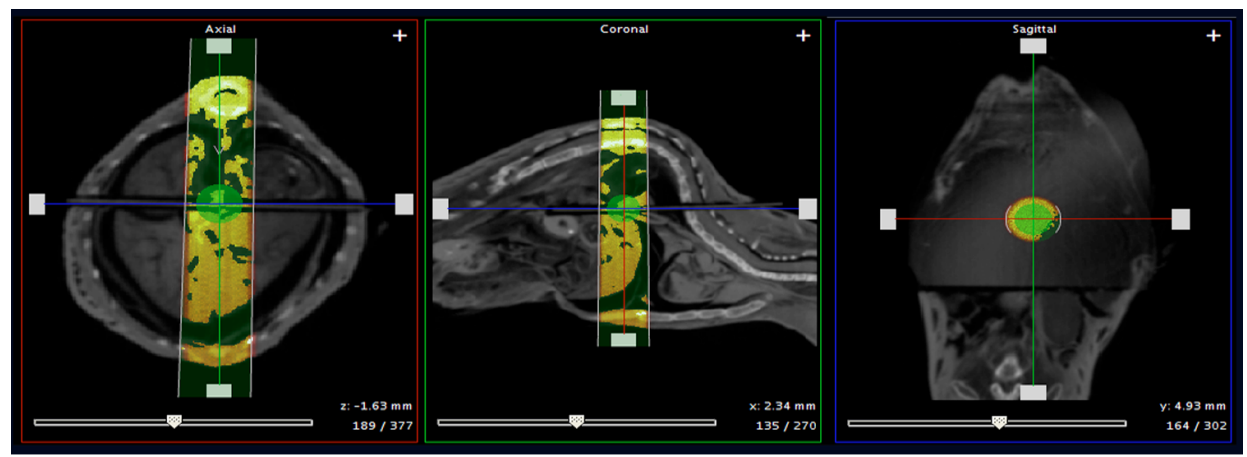

SmART-Plan

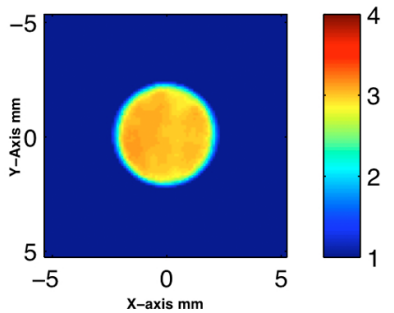

RCF

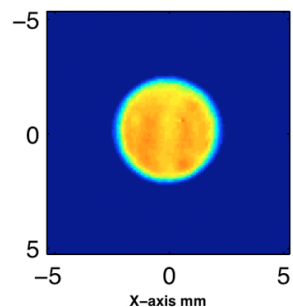

Dose difference (Gy)

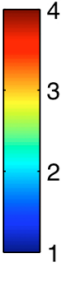

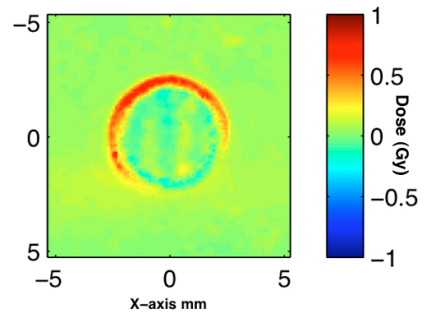

Figure 3.4: 2D CT slices containing target location and dose distribution through the PlastiMouse phantom ()top row). The bottom row shows the calculated and measured dose distributions in the sagittal plane and their difference (calculated - measured).

\subsection{Discussion}

We demonstrated good agreement between dose calculations in SmART-Plan and RCF measurements for simple beam arrangements and for complex multiple-beam multiple-target treatment plans to uniform, heterogeneous and realistic mouse phantoms. For the delivered plans we see agreement within $10 \%$ for the majority of the targeted dose region, with larger differences between $10 \%$ and $20 \%$ near the penumbra regions (figure 3.3 and 3.4 , and figure 3.8 of the supplementary material). Depth dose curves and profiles showed better agreement for the 5 and $15 \mathrm{~mm}$ collimator than the $1 \mathrm{~mm}$ collimator, reflecting the challenge in accurately calculating and delivering a prescribed dose to a target for beam sizes approaching the dose calculation voxel size. The output correction factor for the smallest field is caused by the inability to accurately align the smallest collimator with the location of the complexly shaped focal spot [4]. Dose discrepancies between SmART-Plan and delivery can result from a number of sources:

i CBCT imaging (e.g. reconstruction artefacts); 
ii MC-related dose calculation errors (e.g. poor tissue segmentation or use of human tissues due to poorly known animal tissue composition);

iii Machine-related delivery errors (e.g. collimator misalignments, gantry sag corrections);

iv Measurement-related errors (e.g. absolute dosimetry, RCF);

$\checkmark$ Analysis-related errors (e.g. image registration).

When CBCT is used in radiotherapy patients the conversion of the HU to electron densities, needed for accurate dose calculation, may be inaccurate [10]. The conversion may be problematic due to effects such as beam hardening and photon scatter. However, these effects are mostly relevant for large objects such as radiotherapy patients. For small animals, photon scatter is minimal ( $<5 \%$ for a $5 \mathrm{~cm}$ diameter water cylinder) and beam hardening is insignificant. Other issues such as streaking artefacts could lead to incorrectly assigned tissues. Therefore, more research is needed to establish the suitability of $\mathrm{CBCT}$ imaging for small animal dose calculations.

Small field dosimetry and dose calculation are known to pose problems in radiotherapy with MV beams $[11,12]$ and also in kV dosimetry $[13,14]$. We recently studied in detail the influence of the focal spot shape and its geometric occlusion by small collimators on x-ray output [4]. We found it is difficult to calculate absolute dose for the smallest fields (order of a $\mathrm{mm}$ ) but that for larger fields $\mathrm{MC}$ simulations with a detailed model of the irradiator offer an accuracy of better than $5 \%$. The level of detail in our simulations cannot improve much further but the mechanical robustness and reproducibility of the irradiator can be improved upon. For dosimetry we currently only recommend radiochromic film due to its ease of use, high spatial resolution and good absolute dose measuring capabilities. We use a 3-color readout scheme [9] to avoid pre-scanning the film. We estimate the accuracy of radiochromic dosimetry for the small fields to be about $5 \%$. Others have advocated the use of solid plastic dosimeters [13] but these cannot be used to measure dose closer than $4 \mathrm{~mm}$ to the surface. Regular quality assurance should be part of the operation of small animal irradiators. In more advanced future versions of SmART-Plan we will add multi-modality imaging planning, faster optimised dose calculations, extended DICOM import- and export capabilities, advanced methods for structure contouring, dose analysis, image registration and treatment verification with the onboard imager [5]. 


\subsection{Conclusion}

A dedicated treatment planning system for small animal preclinical work, SmARTPlan, was presented and validated. Good agreement was achieved between measured and calculated dose distributions in various phantoms: uniform, multislab and a realistic plasticized mouse geometry. Achieving good agreement for the smallest field in the study $(1 \mathrm{~mm})$ is challenging, indicating that preclinical studies with the smallest beams may require improvements in the irradiation system. SmART-Plan is a useful tool to support preclinical radiotherapy studies.

\section{Acknowledgments}

Financial support was provided by Precision X-Ray Inc, a Marie Curie grant (PIRG03GA-2008-230911) and a ZonMW grant (40-00506-98-9019). PVG is supported by a PGSD3 scholarship from the Natural Sciences and Engineering Research Council of Canada (NSERC). 


\section{References}

1. Wong J, Armour E, Kazanzides P, lordachita I, Tryggestad E, Deng H, Matinfar M, Kennedy C, Liu Z, Chan T, Gray O, Verhaegen F, McNutt T, Ford E, DeWeese TL. High-resolution, small animal radiation research platform with $\mathrm{x}$-ray tomographic guidance capabilities. Int. J. Radiat. Oncol. Biol. Phys. 2008; 71: 1591-1599. doi: 10.1016/j.jijrobp.2008.04.025.

2. Verhaegen F, Granton P, Tryggestad E. Small animal radiotherapy research platforms. Phys Med Biol 2011; 56: R55-83. doi: 10.1088/0031-9155/56/12/R01.

3. Jensen MD, Hrinivich WT, Jung JA, Holdsworth DW, Drangova M, Chen J, Wong E. Implementation and commissioning of an integrated micro-CT/RT system with computerized independent jaw collimation. Med Phys 2013; 40: 081706. doi: 10.1118/1.4812422.

4. Granton PV, Verhaegen F. On the use of an analytic source model for dose calculations in precision image-guided small animal radiotherapy. Phys. Med. Biol. 2013; 58: 3377. doi: 10.1088/0031-9155/58/10/3377.

5. Granton PV, Podesta M, Landry G, Nijsten S, Bootsma G, Verhaegen F. A combined dose calculation and verification method for a small animal precision irradiator based on onboard imaging. Med Phys 2012; 39: 4155-4166. doi: 10.1118/1.4725710.

6. Boone JM, Velazquez O, Cherry SR. Small-animal X-ray dose from micro-CT. Mol Imaging 2004; 3: 149-158. doi: 10.1162/1535350042380326.

7. Bazalova M, Zhou H, Keall PJ, Graves EE. Kilovoltage beam Monte Carlo dose calculations in submillimeter voxels for small animal radiotherapy. Med Phys 2009; 36: 4991-4999. doi: 10.1118/1.3238465.

8. Ma CM, Coffey CW, DeWerd LA, Liu C, Nath R, Seltzer SM, Seuntjens JP, American Association of Physicists in Medicine. AAPM protocol for 40-300 kV x-ray beam dosimetry in radiotherapy and radiobiology. Med Phys 2001; 28: 868-893. doi: 10.1118/1.1374247.

9. Van Hoof SJ, Granton PV, Landry G, Podesta M, Verhaegen F. Evaluation of a novel triple-channel radiochromic film analysis procedure using EBT2. Phys Med Biol 2012; 57: 4353-4368. doi: 10.1088/0031-9155/57/13/4353.

10. Hatton J, McCurdy B, Greer PB. Cone beam computerized tomography: the effect of calibration of the Hounsfield unit number to electron density on dose calculation accuracy for adaptive radiation therapy. Phys. Med. Biol. 2009; 54: N329. doi: 10.1088/0031-9155/54/15/N01.

11. Aspradakis MM, Byrne JP, Palmans H, Duane S, Conway J, Warrington AP, Rosser K. IPEM report 103: Small field MV photon dosimetry. 2010; Available from: http://inis.iaea.org/Search/search.aspx?orig_q=RN:42026432.

12. Taylor ML, Kron T, Franich RD. A contemporary review of stereotactic radiotherapy: inherent dosimetric complexities and the potential for detriment. Acta Oncol 2011; 50: 483-508. doi: $10.3109 / 0284186 X .2010 .551665$.

13. Newton J, Oldham M, Thomas A, Li Y, Adamovics J, Kirsch DG, Das S. Commissioning a smallfield biological irradiator using point, 2D, and 3D dosimetry techniques. Medical Physics 2011; 38: 6754-6762. doi: 10.1118/1.3663675.

14. Tryggestad E, Armour M, lordachita I, Verhaegen F, Wong JW. A comprehensive system for dosimetric commissioning and Monte Carlo validation for the small animal radiation research platform. Phys Med Biol 2009; 54: 5341-5357. doi: 10.1088/0031-9155/54/17/017. 


\section{Supplementary material}

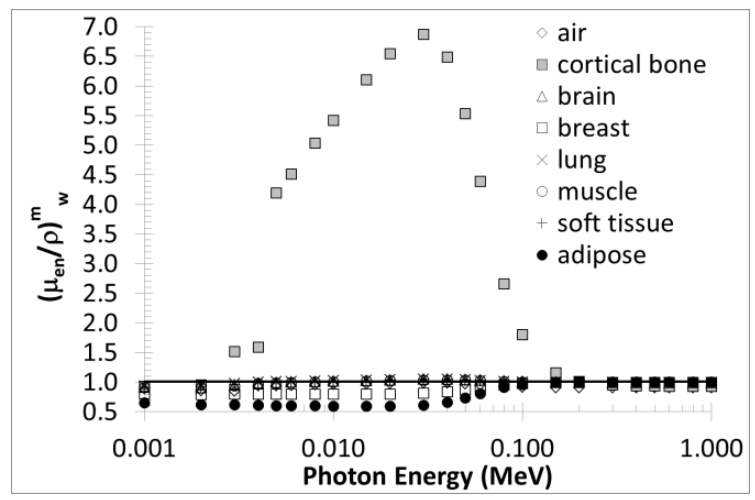

Figure 3.5: Ratio of the photon mass energy absorption coefficients (a measure for absorbed dose) of various tissues to water.

\section{Importance of tissue modeling for $\mathbf{k V}$ dose calculations}

Figure 3.5 shows the ratio of the photon mass energy absorption coefficients (a measure for absorbed dose) of various tissues to water. In 0-225 keV photon energy range the tissue dose differs significantly from water; cortical bone and adipose tissue exhibit the largest deviations. The tissues listed in figure 3.5 are human tissues for which population average tissues compositions are available in the literature [1]. Animal tissue data is not commonly available in great detail.

\section{Screen captures of SmART-Plan}

In figure 3.6 a screen capture of the CT2MD module of SmART-Plan is shown. This module is dedicated to the conversion of $\mathrm{HU}$ to density, and for $\mathrm{HU}$ threshold-based segmentation into various media. The density and medium assignment can be refined further using created structures that can be used to override the initial settings of the CT2MD module.

In figure 3.7 a screen capture of the dose evaluation module of SmART-Plan is shown. The calculated dose distributions and dose-volume histograms of the created structures are shown, and the user can change the dose to the isocentres and adjust beam weights.

\section{Validation of SmART-Plan in a heterogeneous slab phantom}

In figure 3.8 the calculated, measured, and dose difference maps of the validation using the heterogeneous slab phantom setup are shown, measured and validated at the surface, and 10 and $20 \mathrm{~mm}$ below the surface. 
Chapter 3

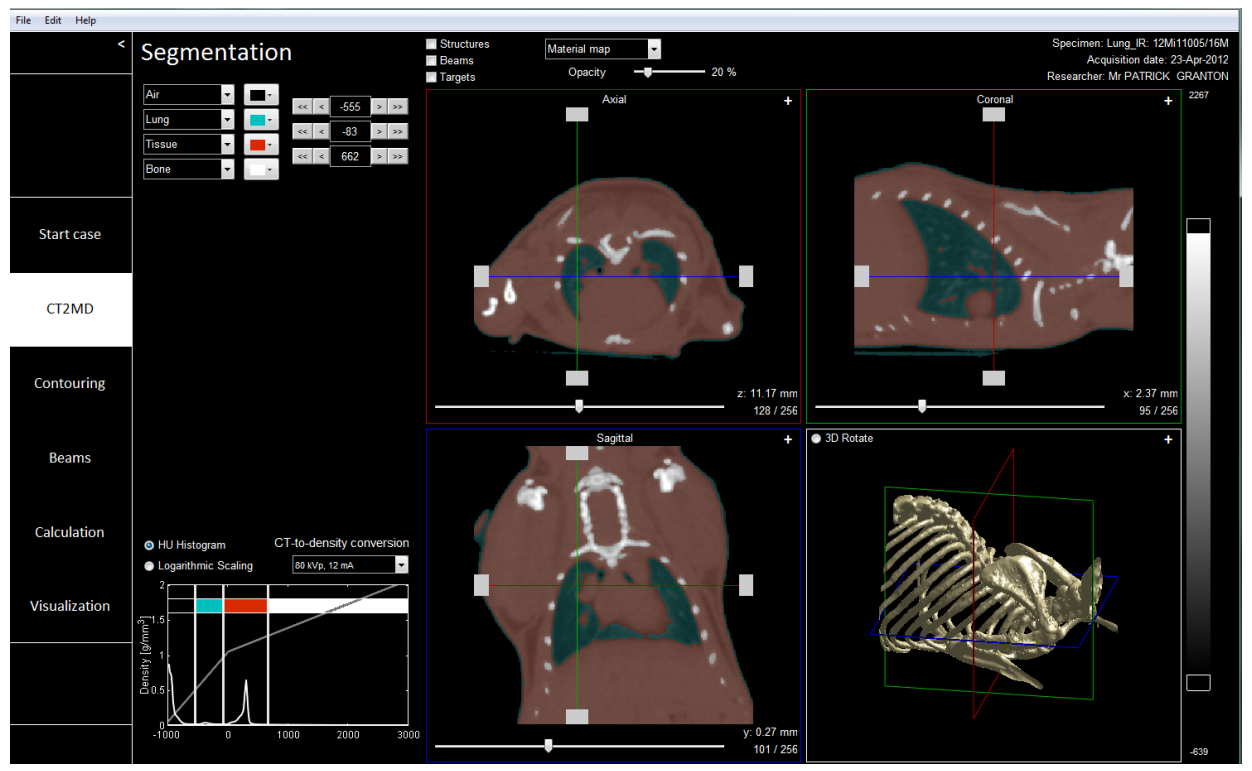

Figure 3.6: Screenshot of SmART-Plan's tissue segmentation module. The HU histogram at bottom left is used to assign densities to voxels, and to segment the tissues. Each vertical band delineated by two vertical white lines represents a different tissue. The three projections show segmented mouse lung tissue. 


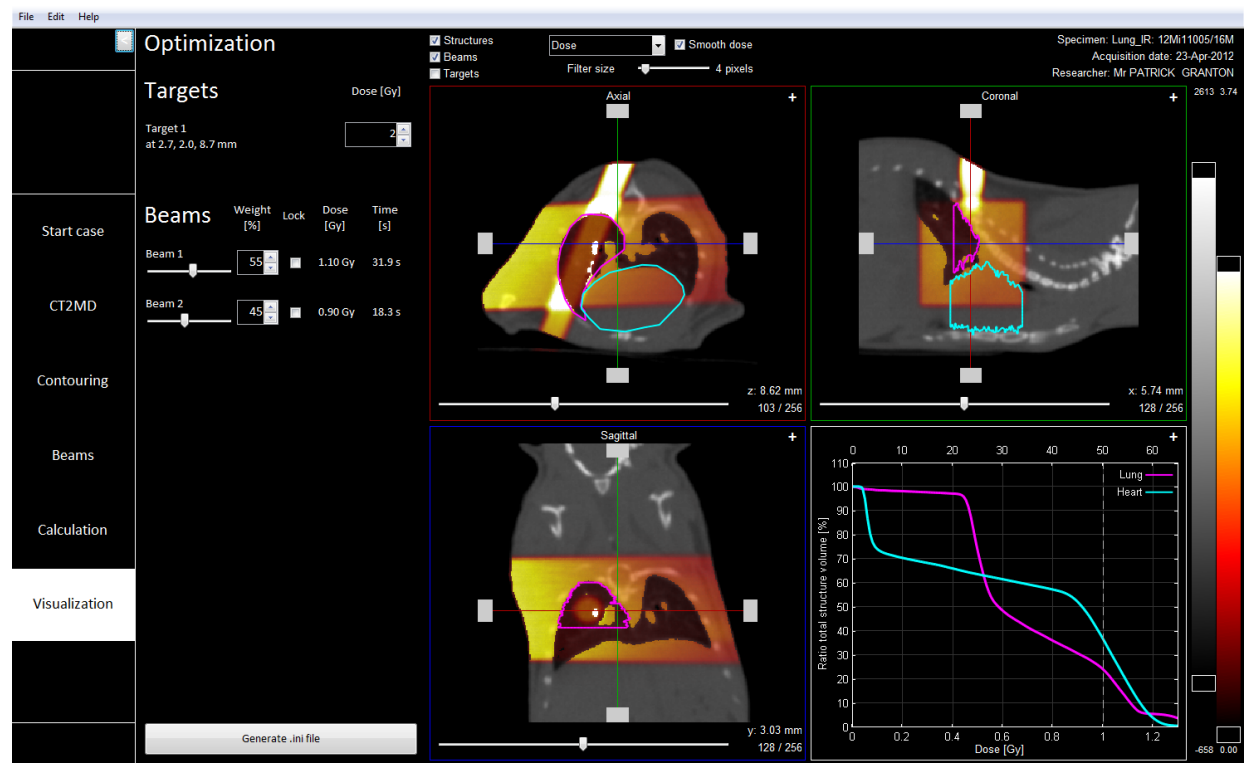

Figure 3.7: Screenshot of SmART-Plan's dose visualization module. The panel labeled Optimization has sliders that allow changing the beam weights, thereby enabling a simple form of plan optimization. The dose volume histogram in two different delineated structures is shown at bottom right. 

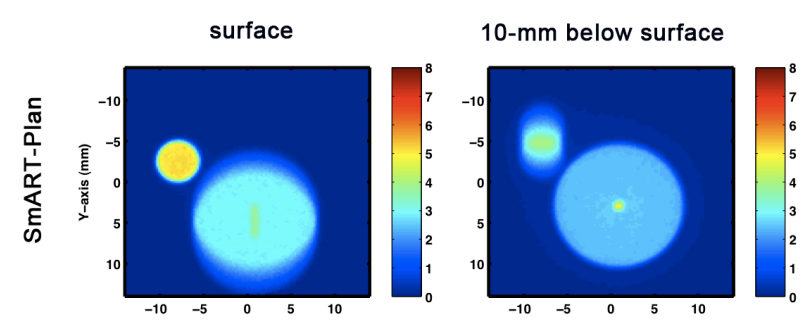

20-mm below surface
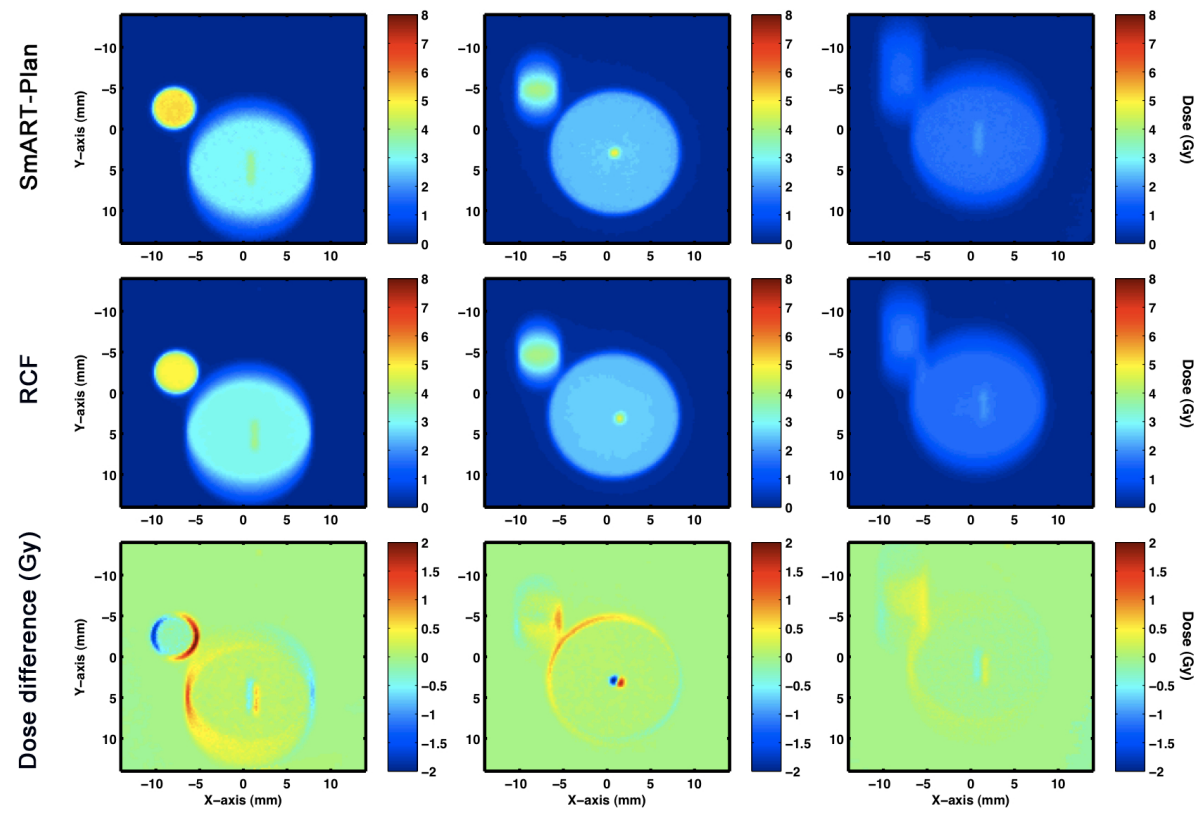

Figure 3.8: Comparison of calculated (top row) and RCF measured (middle row) dose distributions in a solid water/bone/solid water slab phantom. Also shown are the dose differences (calculated - measured) in the bottom row.

\section{References supplementary material}

1. ICRU. 1989. Report 44, Tissue Substitutes in Radiation Dosimetry and Measurement. 


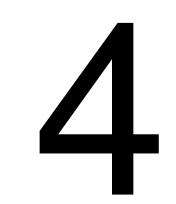

A framework for inverse planning of beam-on times for 3D small animal radiotherapy using interactive multi-objective optimisation

Marleen Balvert*, Stefan J van Hoof*, Patrick V Granton, Daniela Trani, Dick den Hertog, Aswin L Hoffmann, Frank Verhaegen

adapted from

Physics in Medicine and Biology 2015; 60: 5681-5698

${ }^{*}$ contributed equally 


\section{Abstract}

Advances in precision small animal radiotherapy hardware enable the delivery of increasingly complicated dose distributions on the millimeter scale. Manual creation and evaluation of treatment plans becomes difficult or even infeasible with an increasing number of degrees of freedom for dose delivery and available image data. The goal of this work is to develop an optimisation model that determines beam-on times for a given beam configuration, and to assess the feasibility and benefits of an automated treatment planning system for small animal radiotherapy.

The developed model determines a Pareto optimal solution using operator-defined weights for a multiple-objective treatment planning problem. An interactive approach allows the planner to navigate towards, and to select the Pareto optimal treatment plan that yields the most preferred trade-off of the conflicting objectives. This model was evaluated using four small animal cases based on cone beam computed tomography images. Resulting treatment plan quality was compared to the quality of manually optimised treatment plans using dose-volume histograms and metrics.

Results show that the developed framework is well capabable of optimising beam-on times for 3D dose distributions and offers several advantages over manual treatment plan optimisation. For all cases but the simple flank tumour case, a similar amount of time was needed for manual and automated beam-on time optimisation. In this time frame, manual optimisation generates a single treatment plan, while the inverse planning system yields a set of Pareto optimal solutions which provides quantitative insight on the sensitivity of conflicting objectives. Treatment planning automation decreases the dependence on operator experience and allows for the use of class solutions for similar treatment scenarios. This can shorten the time required for treatment planning and therefore increase animal throughput. In addition, this can improve treatment standardisation and comparability of research data within studies and among different institutes. 


\subsection{Introduction}

\subsubsection{Motivation}

Precision small animal image guided radiotherapy is becoming an increasingly important subfield of cancer research. Traditionally, radiobiological studies using small animals were performed using little or no image guidance and crude gross irradiation techniques bearing little resemblance to clinical radiotherapy practice. There is certainly a trend towards orthotopic and genetically engineered tumour models, whereas older work used xenografts [1, 2]. The recent development and commercialisation of ortho-voltage based micro-irradiators enabled studies of cancerous and normal tissues using orthotopic tumour models and advanced irradiation techniques and treatment schemes [3]. These research platforms combine high resolution cone beam computed tomography imaging (CBCT) with precision irradiation capabilities and are revolutionising preclinical radiobiological research [4].

To fully exploit the potential of these devices to deliver complex dose distributions for more advanced studies (e.g. heterogeneous dose boosting or normal tissue complication probability studies), advanced treatment planning software is required, of which the development has been pursued by our group [5]. However, for a growing number of degrees of freedom and available image data, manual creation of beam configurations and dose optimisation becomes increasingly intractable, and the treatment plan quality becomes more dependent on the skills of the operator.

For deep-seated tumours surrounded by organs at risk (OARs), it is difficult to create a treatment plan that yields sufficient dose to the planning target volume (PTV) while sparing OARs [6]. In clinical radiotherapy, mathematical models are used in dedicated treatment planning systems that automatically optimise beam-on times (i.e. monitor units in external beam radiotherapy and dwell times in high-dose rate brachytherapy) for complicated dose distributions (e.g. Shepard et al. [7], Otto [8], De Boeck et al. [9]. Recently, Stewart et al. [10] demonstrated the first beam kernel based preclinical inverse planning model for a 2D target area. Their model optimises beam positions and beam-on times and was validated by experimental data.

The goal of this work is to develop a basic framework for a 3D volume-based inverse treatment planning system, and to test its application in small animal radiotherapy. We present a mathematical model that automatically optimises beam-on times for preclinical small animal radiotherapy using manually created beam configurations as input. Through an interactive procedure, various optimal solutions are generated through 


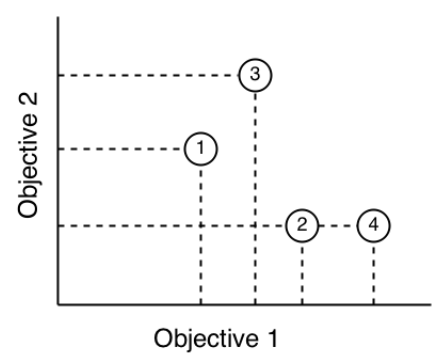

(a)

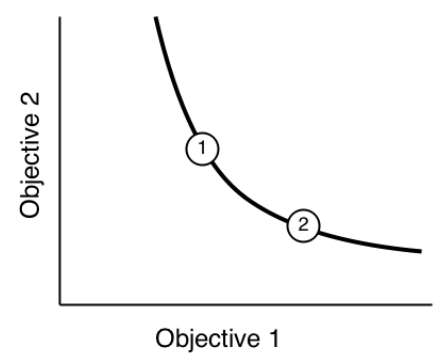

(b)

Figure 4.1: Illustration of Pareto optimality with two objectives. In figure (a), solutions 3 and 4 are not Pareto optimal, solutions 1 and 2 are Pareto optimal, and therefore lie on the Pareto frontier. Figure (b) shows the complete Pareto frontier. Note that the region below the Pareto frontier represents infeasible solutions.

which the operator can navigate towards the most preferred trade-off. The quality of this initial framework is evaluated using data from preclinical treatment cases of a traditional flank tumour irradiation, and deep-seated targets surrounded by OARs. In the remainder of this section, an introduction to existing inverse planning optimisation models in clinical radiotherapy is given, followed by an introduction to the doseoptimisation challenge for preclinical radiotherapy (sections 4.1.2 and 4.1.3, respectively).

\subsubsection{Inverse treatment planning in radiotherapy}

Clinical inverse treatment planning systems for intensity modulated radiotherapy (IMRT) assign a penalty to each tissue structure based on the difference between the intended and delivered minimum, mean or maximum radiation dose. The average penalty over all structures is then minimised [7]. Since this penalty is often a surrogate for the plan evaluation criteria, more recent literature on inverse treatment planning focuses on developing models that optimise radiobiological indices [11, 12] or dose-volume metrics directly $[13,14]$. These more complicated mathematical models are often more computationally intensive, and thus more time consuming, than a penalty-based model.

Treatment planning is a multi-objective problem where a suitable trade-off between the dose to the target and sparing of the OARs needs to be established. Treatment plans for which none of the objectives can be improved without deteriorating at least one other objective are Pareto optimal plans [15], a concept that has been used for IMRT inverse treatment planning [16-18]. Figure 4.1a illustrates the Pareto concept for a 
bi-objective minimisation problem with 4 solution candidates. In this example, solution 3 is not Pareto optimal since both objectives can be improved by applying solution 1 , and solution 4 is not Pareto optimal since objective 1 can be improved by applying solution 2 without worsening objective 2. Solutions 1 and 2 are both Pareto optimal and yield a different trade-off of objectives. In practical applications, many trade-offs and thus many Pareto optimal solutions exist, which together form the Pareto frontier (figure 4.1b). The region below the Pareto frontier represents infeasible solutions.

\subsubsection{Preclinical treatment planning}

There are specific differences for radiation treatment planning and workflow in the preclinical versus the clinical setting. In the preclinical workflow, animals are most often treated based on CBCT data of the animal in treatment position, immediately following image acquisition, see figure 4.2 for an overview. Treatment planning and optimisation should be a fast and easy process because the animal is under anaesthesia. The maximum time an animal can remain under anaesthesia depends on experimental conditions such as the type of anaesthetic applied, the animal model (e.g. nude mice or with fur), the heating applied, and can vary from minutes to hours. The demand for high-throughput studies poses additional limits on the available time for animal treatment. Automation of treatment planning can shorten the treatment preparation and delivery time required per animal, especially when more complex irradiation schemes need to be delivered.

We adopted a penalty-based optimisation approach because it provides good solutions in a relatively short amount of time. Because the choice for the most preferred trade-off is operator and study dependent, an interactive approach was developed that allows the operator to navigate towards the preferred Pareto optimal solution. Once a treatment plan has been created, it can often serve as a class solution for subsequent treatments in the same study, as animals and treatment objectives are often similar in pre-clinical studies.

A Monte Carlo (MC) based dose calculation engine is often used to robustly model photon interactions in the applied orthovoltage photon energy range [6, 19]. In comparison to many dose calculation algorithms used for clinical megavoltage photon energies, such as superposition-convolution algorithms, computational requirements are more demanding for MC approaches which needs to be taken into account by the optimisation model. Furthermore, standardisation and inter-institute comparability of 


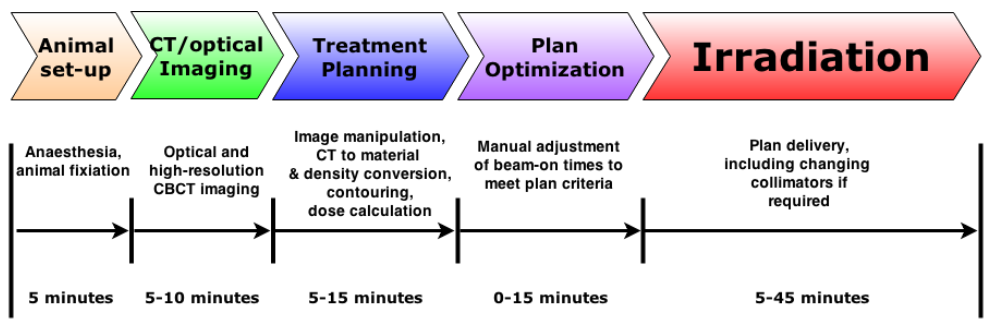

\section{0-90 Minutes}

Figure 4.2: Schematic overview of the different phases in precision small animal radiotherapy. Typical required time spans are provided.

data is especially important in a preclinical research setting, for which automation with the use of treatment planning protocols and class solutions can be valuable. 


\subsection{Methods and materials}

\subsubsection{Inverse planning optimisation model}

\section{Basic optimisation model}

An often used method for finding Pareto optimal solutions to multi-objective optimisation problems is by optimising a weighted sum of the conflicting objectives, where the sum of the non-negative weights equals one [20]. First, we introduce the notation. Sets are indicated using calligraphic letters. The set of organs at risk is denoted by $\mathcal{O} \mathcal{A R}$, and the complete set of tissue structures is denoted by $\mathcal{S}=\mathcal{O} \mathcal{A R} \cup P T V$. Each tissue structure is discretised into voxels, and the set of voxels in structure $s \in \mathcal{S}$ is denoted by $\mathcal{I}_{s} . \mathcal{B}$ denotes the set of beams. The matrix $\dot{d}$ contains the dose rates from all beams to each voxel, where column $\dot{\boldsymbol{d}}_{i}$ is the vector of total dose rates from each beam to voxel $i$, and $\dot{d}_{i b}$ denotes the dose rate to voxel $i$ from beam $b$. The variable $t$ is a vector containing the beam-on times for all beams. The dose to voxel $i$ can now be calculated by $\dot{\boldsymbol{d}}_{i}^{T} \boldsymbol{t}$. The prescribed dose to the target is denoted by $D_{P}$.

For each voxel $i \in \mathcal{I}_{P T V}$, i.e. the set of voxels in the PTV, the variable $v_{i}$ equals the difference between the planned and prescribed dose if the delivered dose is less than $D_{P}$, and 0 otherwise. The optimisation model can now be formulated as follows:

$$
\begin{array}{llrl}
\min _{\boldsymbol{v}, \boldsymbol{t}} & w_{P T V} \frac{1}{\left|\mathcal{I}_{P T V}\right|} \sum_{i \in \mathcal{I}_{P T V}} v_{i}+\left(1-w_{P T V}\right) \sum_{s \in \mathcal{O} \mathcal{A} \mathcal{R}} w_{s} \frac{1}{\left|\mathcal{I}_{s}\right|} \sum_{i \in \mathcal{I}_{s}} \dot{\boldsymbol{d}}_{i}^{T} \boldsymbol{t} & \\
\text { s.t. } & v_{i} \geq D_{P}-\dot{\boldsymbol{d}}_{i}^{T} \boldsymbol{t} & \forall i \in \mathcal{I}_{P T V} \\
& v_{i} \geq 0 & \forall i \in \mathcal{I}_{P T V} \\
& t_{b} \geq 0 & \forall b \in \mathcal{B},
\end{array}
$$

where $w_{s}$ denotes the weight assigned to the objective corresponding to structure $s \in \mathcal{S}$, and $\left|\mathcal{I}_{s}\right|$ denotes the number of voxels in structure $s$. The objective for the PTV is to minimise the difference between the prescribed and delivered dose averaged over all voxels, while the objective for each of the organs at risk is to minimise the total dose over all voxels in this organ. As differences in structure sizes may lead to a more pronounced sparing of large structures, this total dose is divided by the number of voxels in the organ and therefore results in minimising the mean dose to the structure. Note that $w_{P T V}$ denotes the trade-off between the dose to the target and normal tissue sparing, and the remaining weights reflect the relative importance of each of the OARs, where we only consider weights satisfying $\sum_{s \in \mathcal{O} \mathcal{A R}} w_{s}=1$. 


\section{Chapter 4}

This turns out to be a practically convenient approach, as will be further explained in the next section.

\section{Model extensions}

Several additional restrictions on the treatment plan can be included in the model.

The total beam-on time can be limited to $T_{\max }$ :

$$
\sum_{b \in \mathcal{B}} t_{b} \leq T_{\max }
$$

Restrictions on the minimum, mean and maximum dose to the voxels in structure $s \in \mathcal{S}$, denoted by $D_{\min }, D_{\text {mean }}$ and $D_{\max }$, can be included:

$$
\begin{aligned}
\dot{\boldsymbol{d}}_{i}^{T} \boldsymbol{t} \geq L_{s} \quad \forall i \in \mathcal{I}_{s} \quad \forall s \in \mathcal{S} \\
\dot{\boldsymbol{d}}_{i}^{T} \boldsymbol{t} \leq U_{s} \quad \forall i \in \mathcal{I}_{s} \quad \forall s \in \mathcal{S} \\
\frac{1}{\left|\mathcal{I}_{s}\right|} \sum_{i \in \mathcal{I}_{s}} \dot{\boldsymbol{d}}_{i}^{T} \boldsymbol{t} \geq \tilde{L}_{s} \quad \forall s \in \mathcal{S} \\
\frac{1}{\left|\mathcal{I}_{s}\right|} \sum_{i \in \mathcal{I}_{s}} \dot{\boldsymbol{d}}_{i}^{T} \boldsymbol{t} \leq \tilde{U}_{s} \quad \forall s \in \mathcal{S},
\end{aligned}
$$

where $L_{s}$ and $U_{s}$ denote a minimum and maximum allowed $D_{\min }$ and $D_{\max }$ for organ $s$, respectively, and $\tilde{L}_{s}$ and $\tilde{U}_{s}$ denote a minimum and maximum allowed $D_{\text {mean }}$, respectively. Note that restrictions on $D_{\min }$ and minimum $D_{\text {mean }}$ apply to target structures only. Finally, restrictions related to target dose homogeneity limit the absolute difference between $D_{\min }$ and $D_{\max }$ of the PTV to be at most $H$, using auxiliary variables $l$ and $u$ :

$$
\begin{aligned}
& l \leq \dot{\boldsymbol{d}}_{i}^{T} \boldsymbol{t} \quad \forall i \in \mathcal{I}_{P T V} \\
& u \geq \dot{\boldsymbol{d}}_{i}^{T} \boldsymbol{t} \quad \forall i \in \mathcal{I}_{P T V} \\
& u-l \leq H .
\end{aligned}
$$

\section{Navigating through the Pareto frontier}

The operator can navigate through the Pareto frontier by adjusting the weights $w_{P T V}$ and $w_{s}, s \in \mathcal{O} \mathcal{A R}$. The trade-off between dose to the PTV and sparing of the OARs is the most important trade-off. For this, the operator needs to run the model for a range of values for $w_{P T V}$, e.g. from 0 to 1 with a step size of 0.1 , while fixing the OAR weights. Based on those results, the planner can narrow the range and decrease the step size to find a plan that best matches the preferred trade-offs. Thereafter, the plan 
can be fine-tuned by adjusting the weights of the OARs, or by choosing a range of weights for one of the OARs to compare different trade-offs. Varying OAR weights may influence dose to the PTV, for which it may be necessary to re-adjust the weight for the PTV versus OARs. An example of such an optimisation process can be found in the supplementary material.

\subsubsection{Treatment planning}

To create beam configurations and perform dose calculations, a research version of the small animal radiotherapy treatment planning system SmART-Plan (version 2.0, Precision X-ray Inc, North Branford, CT) was used in which the optimisation model was integrated [19]. The optimisation model was solved using CPLEX 12.6 Optimiser (IBM corporation, Somers, USA) which was interfaced to MATLAB Release 2012b (The MathWorks, Inc., Natick, USA), on a computer with an Intel i7-2670 QM processor. All cases were planned for irradiation with an X-RAD 225Cx (Precision X-Ray Inc, North Branford, CT), using a $225 \mathrm{kVp}$ (0.32 mm Cu filtration) spectrum [21].

A forward treatment planning approach was used in which beams are positioned manually and shown as overlay over CBCT image data to provide an estimate of the expected dose distribution. SmART-Plan uses the Monte Carlo (MC) code DOSXYZnrC [22] to perform dose calculations, using a validated MC source model in voxelised geometries based on the CBCT image data [23]. For MC dose calculations both the material (i.e. cross-section data) and mass density are required and were assigned on a voxel-by-voxel basis according to the Hounsfield Units (HU) [5]. The conversion of $\mathrm{HU}$ to material type and density was performed based on a 4-material HU threshold scheme and a density calibration curve that was established using materials of known composition and density. The material classes were air, lung, soft tissue, and cortical bone (ICRU report 44 [24]). The HU thresholds were established based on visual inspection of the CT data. Dose calculations were run to a statistical uncertainty of $<3 \%$ in the target region, using energy transport cutoffs for the photon and electron transport of 10 and $100 \mathrm{keV}$, respectively. Dose was scored as dose-to-medium, transport-in-medium.

\subsubsection{Treatment planning and delivery times}

Operators recorded the time that was required for the optimisation and non-optimisation part of the treatment planning process. The non-optimisation part of the treatment planning includes time needed to import and manipulate image 
data in SmART-Plan, assign materials and mass densities, delineate structures and perform dose calculations. The time required to perform the dose calculations was recorded for all cases and includes the time required to create the input data for the MC calculations and import resulting dose distributions into SmART-Plan. The time for manual optimisation only includes adjusting beam weights and evaluating the intermediate and final dose distributions. For automated optimisation, these times include the iterative process of adjusting weights for the PTV and OARs, running the optimisation algorithm, and evaluating intermediate and final dose distributions. The automated and manual approaches use the same dose distributions as input. All treatment plans were delivered in the shortest time possible by using the highest dose rate the X-RAD 225Cx provides, by ordering beams from low to high gantry angle and by initializing plan delivery with the gantry positioned at the angle of the first beam. The time to deliver a treatment plan includes beam-on times, the time needed to switch the X-ray tube on and off, and time to reposition the gantry.

\subsubsection{Treatment cases}

Four treatment scenarios were created based on a rat with a large flank tumour using a CBCT image with isotropic voxel spacing of $0.4 \mathrm{~mm}$. For the first case, the flank tumour was targeted and both kidneys, spine, and gastrointestinal tract (GI) were delineated as OARs. Two parallel opposed $40 \times 40 \mathrm{~mm}$ beams at a 12 degree offset from the vertical axis, linked to a single machine isocentre, were used.

For the second case, the left kidney was considered as PTV and the right kidney, spine, and GI were included as OARs. A beam configuration consisting of four beams linked to three machine isocentres using $10 \times 10 \mathrm{~mm}$ beams was created. Two beams are so-called partial arc or dynamic beams, which are planned to deliver radiation while the machine gantry rotates and the animal stage remains stationary. The two remaining beams were static parallel opposed anterior-posterior posterior-anterior beams.

For the third and fourth case, a crescent-shaped fictitious PTV with a volume of 946 $\mathrm{mm}^{3}$ was created wrapped around the spine, and the two kidneys, spine and Gl were used as OARs. Case 3 and 4 were different only with regard to the beam configurations. For case 3 , six $10 \times 8 \mathrm{~mm}$ rectangular beams were linked to five machine isocentres, one isocentre above the spine close to the skin with two parallel opposed lateral-medial medial-lateral beams, and two isocentre at each side of the spine. The beams were positioned with angular separations of 45 degrees. For case 4 , twelve 


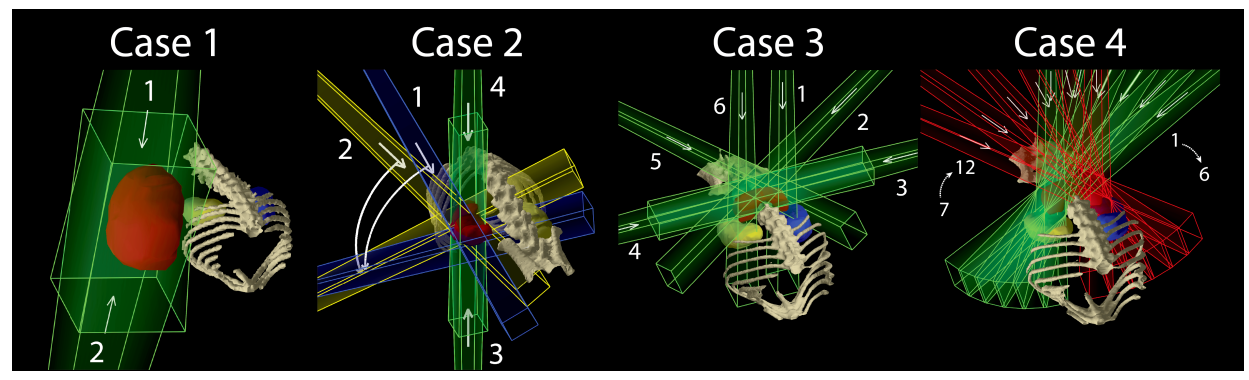

Figure 4.3: Beam configurations of the four treatment cases. Beam directions are indicated with arrows. Dynamic arc beams are illustrated by drawing the initial and final beam positions, connected with curved arrows. Beam index numbers correspond to the beam-on time data in table 4.2.

$10 \times 8 \mathrm{~mm}$ rectangular beams linked to two machine isocentres next to the spine were used. The beams were positioned with angular separations of 10 degrees. The beam configurations of cases 3 and 4 were both symmetrical with a virtual mirror plane along the spine of the animal.

Since the flank tumour was not used as the planning target for cases 2,3 and 4 , the tumour was cropped from the CBCT image data for those cases. This did not affect the treatment plans or the optimisation model as beams did not traverse the flank tumour before reaching their target. Because this work concerns an in silico planning study, margins of $0 \mathrm{~mm}$ were taken for sub-clinical disease spread and uncertainties in treatment delivery were not taken into account. Therefore, the PTV was equal to the gross tumour volume for all cases, and dose was planned to the PTV. See figure 4.3 for illustrations of the beam configurations and PTVs for all cases.

For all cases, a prescription dose of 8 Gy to the PTV was used, and a mean dose to the PTV between 8 and 10 Gy was set as a constraint. No constraints on the minimum and maximum dose and dose heterogeneity were used. Note that the dose to OARs is only included in the penalty objective. The manual beam-on time optimisations were performed by a different operator than the automated optimisations to prevent influence of prior knowledge of the treatment plans on our results. As different planners may have different trade-off preferences, the preferences of the second planner need to be matched to those of the first to obtain comparable treatment plans. Therefore, the planner using automated beam-on time optimisation aimed for achieving the same $V_{95 \%}$ as obtained with manual optimisation. Here, $V_{95 \%}$ is defined as the fraction of the target volume receiving at least $95 \%$ of the prescribed dose. 


\subsection{Results}

\subsubsection{Dose distributions}

An overview of the dose distributions of each case is shown in figure 4.4. Dose and volume metrics for the manually and automatically optimised treatment plans for all four cases are presented in table 4.1 and dose-volume histograms (DVHs) are presented in figure 4.5. The results show that the two methods yield dose distributions of similar quality. For case 1 the DVHs of the manual and automated approaches are fully overlapping, as finding an optimal treatment plan for this case is straightforward. The main difference between the plans for case 2 was a lower target dose homogeneity when using the automated optimisation, as can be observed from the DVHs and the relative standard deviation, $\sigma_{D} / D_{\text {mean }}$, defined as the standard deviation of the dose to all voxels in the PTV divided by the mean dose of the PTV. The target dose homogeneity for case 3 was better when using automated plan optimisation, and for case 4 hardly any difference can be observed. For case 3 , the minimum dose to the hottest $5 \%$ of the PTV, $D_{5 \%}$ (PTV), was lower when using automated plan optimisation and sparing of the spine was much improved. This came at the cost of a slightly higher $D_{5 \%}(\mathrm{Gl})$. For case 4 , the operators had slightly different trade-off preferences: the dose to the Gl and the kidneys was higher for the automatically optimised plan, but the dose to the spine was much lower.

\subsubsection{Trade-off}

The Pareto approach allowed the operator to choose from various trade-offs. Figure 4.6 presents a set of possible treatment plans obtained by automated optimisation using varying optimisation weights, for all four cases. For example, for case 1 , an increase in $V_{95 \%}$ (PTV) comes at a cost of an increase in $D_{1 \%}(\mathrm{Gl})$. For case 2, a slight increase in $D_{5 \%}$ for the Gl from 5.9 to 6.1 Gy allows for an increase in $V_{95 \%}$ of the PTV from $60 \%$ to $66 \%$. Also increasing $V_{95 \%}$ for case 3 comes at low cost as shown in figure 4.6, while the remaining DVH parameters remained approximately constant. For case 4 , roughly every additional percentage of $V_{95 \%}$ (PTV), required an increase in $D_{5 \%}$ (spine) of approximately 0.8 Gy in the displayed range.

\subsubsection{Treatment planning and delivery times}

The beam-on times and time spent on optimisation and delivery of all treatment plans for the manual and automated approach are shown in table 4.2. The time needed for 


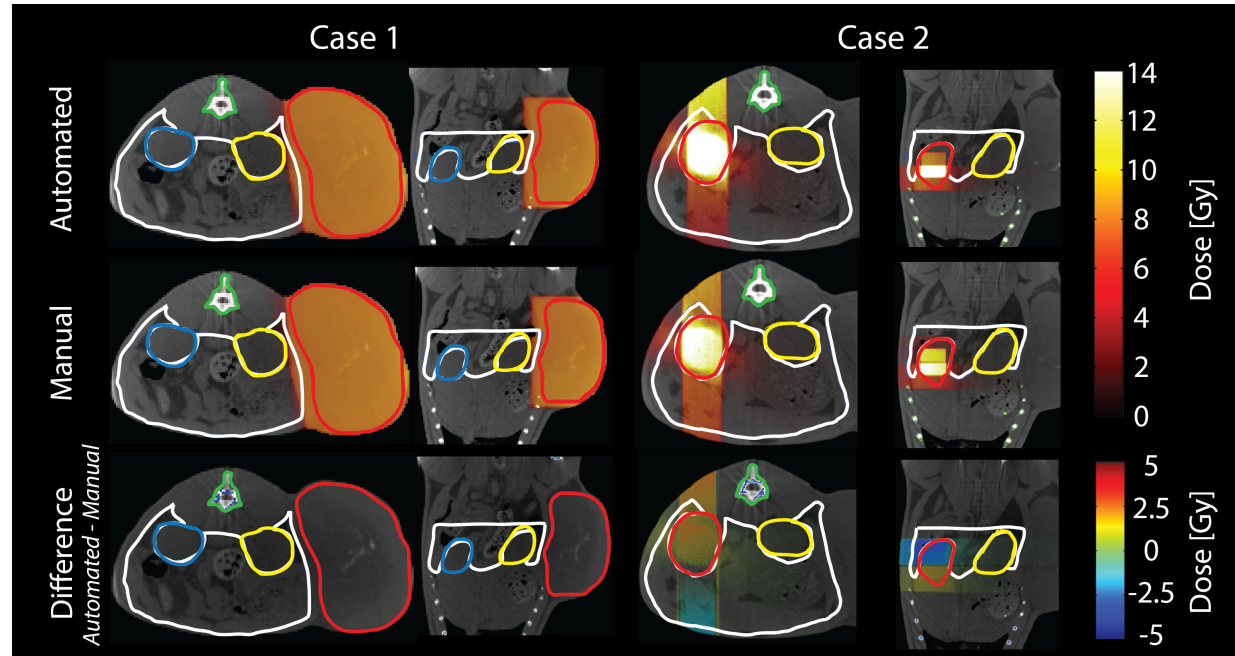

Case 3

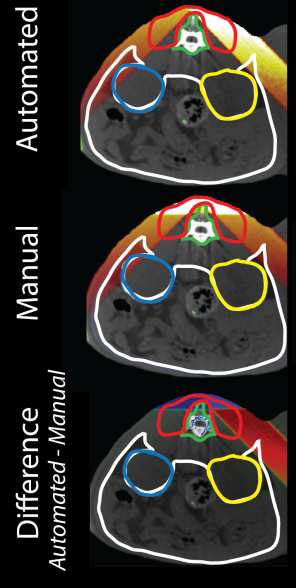

Case 4

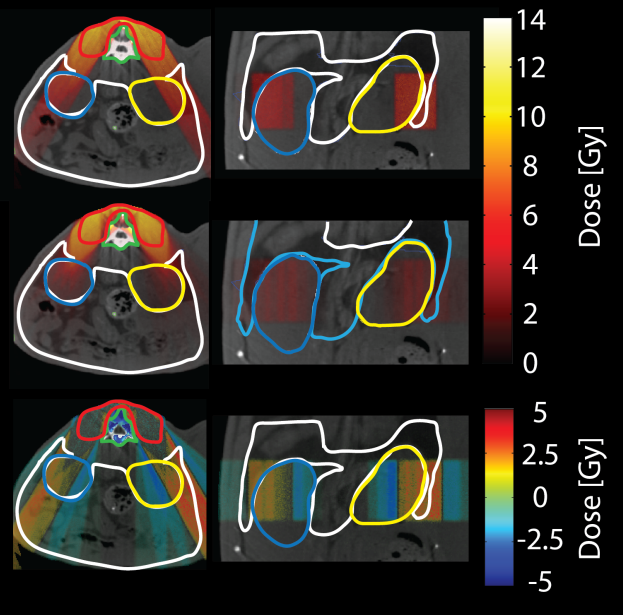

Figure 4.4: Overview of the dose distributions resulting from automated and manual treatment plan optimisation and their differences. Window and level settings for visualisation of both the CT and overlaid dose distributions are equal for all images. PTVs are delineated in red, the right kidney in yellow, the left kidney in blue, the Gl in white, and the spine in green. 
Table 4.1: Dose-volume metrics using manual (man) and automatic (aut) beam-on time optimisation. Dose values are given in Gy, and $V_{95 \%}$ is given as a percentage of the total PTV volume. $\sigma_{D} / D_{\text {mean }}$ is the standard deviation of the dose to the voxels in the PTV divided by the mean PTV dose. PTV=planning target volume.

\begin{tabular}{|c|c|c|c|c|c|c|c|c|}
\hline & \multicolumn{2}{|c|}{ Case 1} & \multicolumn{2}{|c|}{ Case 2} & \multicolumn{2}{|c|}{ Case 3} & \multicolumn{2}{|c|}{ Case 4} \\
\hline & man & aut & man & aut & man & aut & man & aut \\
\hline \multicolumn{9}{|l|}{ PTV } \\
\hline$V_{95 \%}(\%)$ & 100.0 & 100.0 & 61.2 & 61.7 & 68.0 & 68.1 & 83.8 & 84.3 \\
\hline$D_{\min }(\mathrm{Gy})$ & 7.6 & 7.6 & 0.0 & 0.0 & 0.0 & 0.0 & 0.5 & 0.4 \\
\hline$D_{95 \%}$ (Gy) & 7.9 & 7.9 & 1.1 & 0.5 & 0.4 & 0.4 & 5.9 & 5.4 \\
\hline$D_{\text {mean }}(\mathrm{Gy})$ & 8.2 & 8.2 & 8.3 & 8.2 & 9.9 & 8.7 & 8.3 & 8.1 \\
\hline$D_{5 \%}$ (Gy) & 8.5 & 8.5 & 13.8 & 15.6 & 19.8 & 15.5 & 9.6 & 9.4 \\
\hline$D_{\max }(\mathrm{Gy})$ & 9.5 & 9.5 & 16.1 & 18.8 & 30.3 & 26.2 & 11.1 & 15.2 \\
\hline$\sigma_{D} / D_{\text {mean }}$ & 0.0 & 0.0 & 0.47 & 0.58 & 0.68 & 0.61 & 0.16 & 0.18 \\
\hline \multicolumn{9}{|l|}{ left kidney } \\
\hline$D_{5 \%}$ (Gy) & 0.2 & 0.2 & $\mathrm{n} / \mathrm{a}$ & $\mathrm{n} / \mathrm{a}$ & 5.7 & 5.6 & 5.5 & 6.1 \\
\hline$D_{1 \%}$ (Gy) & 0.2 & 0.2 & $\mathrm{n} / \mathrm{a}$ & $\mathrm{n} / \mathrm{a}$ & 6.3 & 6.2 & 6.7 & 6.6 \\
\hline \multicolumn{9}{|l|}{ GI } \\
\hline$D_{5 \%}$ (Gy) & 4.0 & 4.0 & 6.9 & 5.9 & 0.3 & 0.4 & 2.4 & 4.4 \\
\hline$D_{1 \%}$ (Gy) & 7.9 & 7.9 & 8.9 & 8.4 & 5.8 & 6.3 & 4.3 & 6.3 \\
\hline \multicolumn{9}{|l|}{ right kidney } \\
\hline$D_{5 \%}$ (Gy) & 1.1 & 1.3 & 1.6 & 1.8 & 0.3 & 0.4 & 4.7 & 6.1 \\
\hline$D_{1 \%}$ (Gy) & 2.8 & 2.7 & 2.0 & 2.3 & 0.3 & 0.5 & 5.6 & 6.4 \\
\hline \multicolumn{9}{|l|}{ spine } \\
\hline$D_{5 \%}$ (Gy) & 1.3 & 1.3 & 0.3 & 0.3 & 0.8 & 0.6 & 7.4 & 3.0 \\
\hline$D_{1 \%}$ (Gy) & 1.5 & 1.5 & 0.4 & 0.4 & 10.7 & 1.0 & 15.7 & 9.6 \\
\hline
\end{tabular}




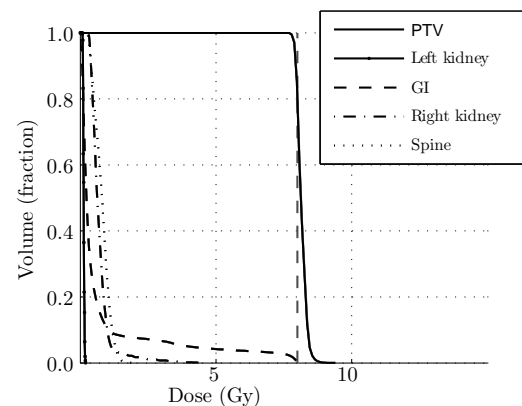

(a) Case 1

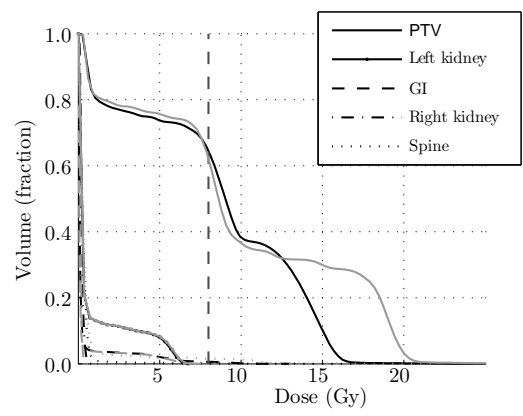

(c) Case 3

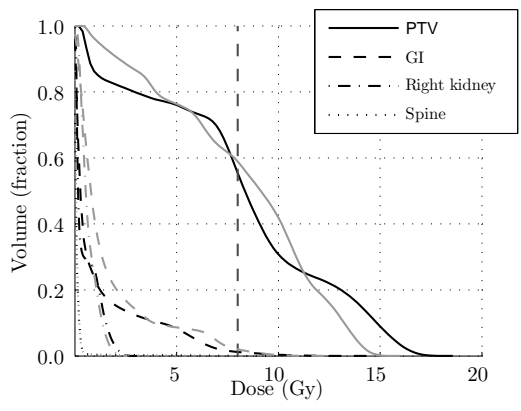

(b) Case 2

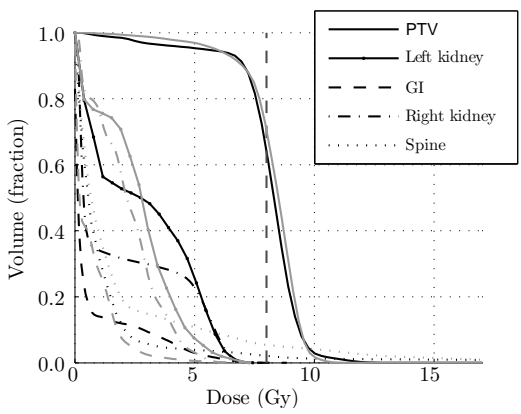

(d) Case 4

Figure 4.5: Dose-volume histograms obtained using manual (gray) and automated (black) beam-on time optimisation. The vertical dashed line indicates the prescription dose.

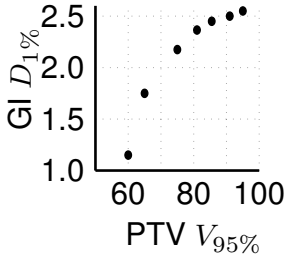

(a)

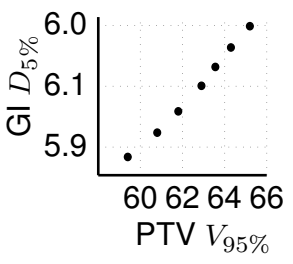

(b)

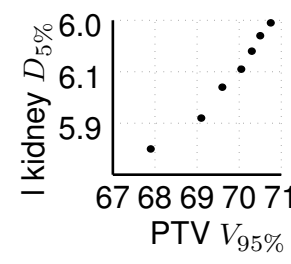

(c)

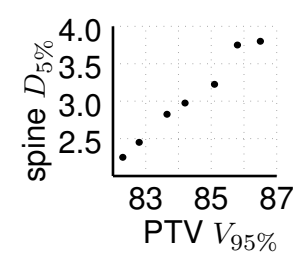

(d)

Figure 4.6: A trade-off between target coverage (PTV $V_{95 \%}$ ) as a percentage of the total volume and sparing of (a) the GI $\left(D_{1 \%}\right)$ for case 1, (b) the Gl $\left(D_{5 \%}\right)$ for case 2, (c) the left kidney $\left(D_{5} \%\right)$ for case 3 and $(\mathrm{d})$ the spine $\left(D_{5 \%}\right)$ for case 4 , measured in Gy. 
optimisation only was similar for the manual and automated procedures for cases 2 , 3 and 4 . For case 1, the size of the tumour results in a large number of voxels for the PTV and corresponding variables. This increases the computation time for the optimisation algorithm, whereas manual optimisation is straightforward and requires very little time.

For automatic optimisation, a single treatment plan was optimised within approximately 20 seconds. Searching for the most preferred Pareto optimal solution required a similar amount of time as generating a single final treatment plan when optimising manually. As a result, within the amount of time needed for manually optimising a single treatment plan, multiple Pareto optimal treatment plans were generated with automatic optimisation.

The plan delivery times for the automatically optimised plans were lower due to the exclusion of many beams, and were further decreased by the somewhat lower total beam-on times when using automated optimisation. For example, the total beam-on time for case 4 reduced by 20 seconds when using automatic treatment planning. In addition, the total time needed for plan delivery was reduced by another 26 seconds due to the smaller number of beams used. This resulted in a total plan delivery time that was reduced from 7:26 minutes to 6:40 minutes. However, the lower total beamon times and exclusion of beams were not a general feature as they were not set as one of the objectives.

The non-optimisation part of treatment planning required 23:20, 24:03, 23:53 and $26: 13$ minutes for case $1,2,3$ and 4 , respectively. Of this non-optimisation part, the dose calculations required 5:20, 4:03, 2:53 and 4:13 minutes for case 1, 2, 3 and 4, respectively. 
Table 4.2: Beam-on times, time spent on plan optimisation and time required to deliver treatment plans for the manual (man) and automated (aut) optimisation approaches.

\begin{tabular}{|c|c|c|c|c|c|c|c|c|}
\hline \multirow[b]{2}{*}{ beam [-] } & \multicolumn{2}{|c|}{ Case 1} & \multicolumn{2}{|c|}{ Case 2} & \multicolumn{2}{|c|}{ Case 3} & \multicolumn{2}{|c|}{ Case 4} \\
\hline & $\operatorname{man}$ & aut & man & aut & man & aut & $\operatorname{man}$ & aut \\
\hline 1 & $1: 39$ & $1: 35$ & $1: 34$ & $0: 00$ & $0: 00$ & $0: 00$ & $0: 30$ & $0: 00$ \\
\hline 2 & $1: 37$ & $1: 41$ & $2: 57$ & $3: 31$ & $3: 52$ & $6: 19$ & $0: 29$ & $0: 00$ \\
\hline 3 & & & $1: 12$ & $0: 00$ & $2: 18$ & 0:04 & $0: 30$ & $0: 00$ \\
\hline 4 & & & $2: 54$ & $4: 20$ & $2: 17$ & $0: 00$ & $0: 33$ & $3: 10$ \\
\hline 5 & & & & & $3: 54$ & $3: 50$ & $0: 36$ & $0: 00$ \\
\hline 6 & & & & & $0: 00$ & $0: 01$ & $0: 41$ & $0: 00$ \\
\hline 7 & & & & & & & $0: 41$ & $0: 00$ \\
\hline 8 & & & & & & & $0: 37$ & $0: 41$ \\
\hline 9 & & & & & & & $0: 33$ & $2: 12$ \\
\hline 10 & & & & & & & $0: 31$ & $0: 00$ \\
\hline 11 & & & & & & & $0: 30$ & $0: 00$ \\
\hline 12 & & & & & & & $0: 29$ & $0: 17$ \\
\hline total beam-on time & $3: 16$ & $3: 16$ & $8: 37$ & $7: 51$ & $12: 21$ & $10: 14$ & $6: 40$ & $6: 20$ \\
\hline plan optimisation & $0: 30$ & $2: 05$ & $4: 00$ & $4: 30$ & $4: 30$ & $5: 00$ & $5: 00$ & $5: 00$ \\
\hline \multicolumn{9}{|l|}{ plan delivery } \\
\hline total beam-on time & $3: 16$ & $3: 16$ & $8: 37$ & $7: 51$ & $12: 21$ & $10: 13$ & $6: 40$ & $6: 20$ \\
\hline non-beam-on time & $0: 31$ & $0: 31$ & $0: 40$ & $0: 14$ & $0: 32$ & $0: 33$ & $0: 46$ & $0: 20$ \\
\hline total & $3: 47$ & $3: 47$ & $9: 17$ & 8:05 & $12: 53$ & $10: 46$ & $7: 26$ & $6: 40$ \\
\hline
\end{tabular}

Note: Beam-on times are provided per beam, and the total beam-on time per treatment plan. The time required to deliver treatment plans is split into the total beam-on time for the plan, and time to reposition the gantry and switch the x-ray tube on and off, referred to as non-beam-on time. All times are denoted as min:s. 


\subsection{Discussion}

This work shows that the developed framework for precision small animal radiotherapy inverse treatment planning is well capable of optimising beam-on times for 3D dose distributions and offers several advantages over manual treatment plan optimisation. The applied Pareto approach provides quantitative insights on trade-offs for mutually conflicting optimisation objectives. Automation in pre-clinical treatment planning decreases the dependence on the skills of the operator, allows for the use of class solutions, and eases the creation and evaluation of more complex beam configurations.

In precision image guided small animal radiotherapy, most operators are not experts in treatment planning, and would especially benefit from automation of the treatment planning process. Adding protocols and class solutions for specific studies or treatments can reduce valuable planning time significantly since different animals in preclinical studies often have similar anatomical characteristics. From experience in clinical IMRT it is known that for similar cases, the optimal weight vectors are similar as well [25]. This form of standardisation can improve comparability of animal data within one study, and even among different institutions. Traditionally, beam-on times are fixed for all animals within one study, which does not necessarily yield similar dose distributions, whereas the optimisation model will provide animal specific beam weights resulting in a similar trade-off between PTV and OAR doses. Furthermore, the use of automated optimisation allows for the delivery of more complicated treatment schemes which are required for advanced radiobiological studies such as heterogeneous dose boosting and dose painting [26].

The concept of Pareto optimality allows the planner to assess possible trade-offs by solving the model for various sets of penalty weights. This provides the operator with quantitative data on the cost of improving one objective in relation to deteriorating other objectives. For example, figure $4.6 \mathrm{~b}$ shows that improving $V_{95 \%}$ (PTV) requires a minor increase in maximum OAR dose for case 2. In contrast, the operator who used manual optimisation was not aware of this quantitative sensitivity information, and thus did not improve PTV coverage.

The penalty-based objectives used in our model are surrogates for dose-volume characteristics. A Pareto-optimal treatment plan that is optimized with respect to penalty functions is thus not necessarily Pareto-optimal with respect to dose-volume metrics. On the other hand, directly optimizing dose-volume metrics is computationally expensive [14], which is undesirable for small animal studies where strict time constraints 
on treatment planning and animal throughput often exist. Since the penalty-based optimisation model performs well for our application and is currently used in clinical radiotherapy, we adopted that approach.

An interesting observation is the small number of beams selected by the optimisation model. Holm et al. [27] argue that for high-dose rate brachytherapy, the number of positive dwell times is restricted by the number of voxels that receive exactly the prescribed dose. We often observe fewer active beams than voxels receiving the prescribed dose, implying that the findings by Holm et al. do not fully explain the result for our cases. A more intuitive explanation is found when looking at the beam configurations. For cases 3 and 4, the model avoids the use of beams that pass through the spine, which is caused by a high weight factor for the spine for both cases. The exclusion of beams by the optimisation model results in a lower plan delivery time, which is slightly more decreased by the lower total beam-on times for the automated optimised plans as shown in table 4.2.

As we only include beam-on times as degrees of freedom in this first study on 3D preclinical dose optimisation, the achievable quality of the final dose distributions is highly dependent on the a priori determined beam configuration. Especially the lack of degrees of freedom for shaping and positioning of the beams can quickly result in problems with PTV coverage or infeasible optimisation constraints. Including optimisation of the beam configuration can strongly improve treatment plan quality, as is illustrated by the results from cases 3 and 4 presented in figure 4.5. A higher quality plan was achieved with the 12-beam configuration of case 4 in comparison with the 6-beam configuration of case 3 , despite the fact that the optimal solution used only 4 of the 12 beams. Future hardware improvements such as jaws or multi-leaf collimators similar to those used in clinical radiotherapy could improve plan quality even further. With such additional degrees of freedom, automated plan optimisation becomes essential.

The presented model needs operator-defined weights that have no direct interpretation. This complicates the a priori choice of weights. As a result, in the first iterations the treatment plans need to be optimised for a wide range of weight vectors. As noted before, the use of class solutions can reduce this workload. Moreover, the atomic composition of the defined anatomical structures can strongly influence calculated dose values in the $\mathrm{kV}$ photon energy range when scoring dose-to-medium (e.g. for calcium-rich bone), which is the common dose-scoring method in Monte Carlo based engines. This in turn influences the weight vector required for a certain treatment plan. 
The option in the dose engine to score dose-to-water would improve the consistency of the weight vector across different animals.

To our knowledge, Stewart et al. [10] are the only group that has investigated the possibilities of inverse treatment planning for small animal radiotherapy. Our work differentiates through exclusion of the optimisation of beam positions, but is more applicable to realistic animal studies because it focuses on optimising 3D dose distributions based on CBCT data of different pre-clinical cases instead of 2D dose distributions to radiochromic film.

\subsection{Conclusion}

In this work, we developed a basic framework for multi-objective beam-on time optimisation for 3D dose distributions in small animal radiotherapy. Results show that this model is capable of optimising treatment plans to a similar quality as achieved with manual optimisation, and offers several advantages over manual treatment optimisation for orthotopic tumours. Automated treatment plan optimisation allows for improved standardisation and decreases the dependence of plan quality on operator skills. The Pareto approach gives quantitative insight in the sensitivity of tradeoffs of conflicting objectives. Automated treatment plan optimisation can benefit animal throughput through the use of class solutions and by lowering treatment delivery time.

\section{Acknowledgements}

We would like to thank Dr. Davide Fontanarosa and Mark Podesta for their valuable input during discussions. Support for the purchase and operation of the microirradiator was provided by a Marie Curie grant (PIRG03-GA-2008-230911), a ZonMW grant (40-00506-98-9019) and by the GROW research institute. Patrick V. Granton was supported by a Kootstra Fellowship from CRISP, Centre for Research Innovation, Support and Policy of Maastricht University, and by a PGSD3 scholarship from the Natural Sciences and Engineering Research Council of Canada (NSERC). 


\section{References}

1. Tillner $F$, Thute $P$, Bütof $R$, Krause $M$, Enghardt $W$. Pre-clinical research in small animals using radiotherapy technology-a bidirectional translational approach. Z Med Phys 2014; 24 : 335-351. doi: 10.1016/j.zemedi.2014.07.004.

2. Butterworth KT, Prise KM, Verhaegen F. Small animal image-guided radiotherapy: status, considerations and potential for translational impact. $\mathrm{Br} J$ Radiol 2015; 88: 20140634. doi: 10.1259/bjr.20140634.

3. Granton PV, Dubois L, van Elmpt W, van Hoof SJ, Lieuwes NG, De Ruysscher D, Verhaegen F. A longitudinal evaluation of partial lung irradiation in mice by using a dedicated imageguided small animal irradiator. Int. J. Radiat. Oncol. Biol. Phys. 2014; 90: 696-704. doi: 10.1016/j.jijrobp.2014.07.004.

4. Verhaegen F, Granton P, Tryggestad E. Small animal radiotherapy research platforms. Phys Med Biol 2011; 56: R55-83. doi: 10.1088/0031-9155/56/12/R01.

5. Verhaegen F, van Hoof S, Granton PV, Trani D. A review of treatment planning for precision image-guided photon beam pre-clinical animal radiation studies. Z Med Phys 2014; 24 : 323-334. doi: 10.1016/j.zemedi.2014.02.004.

6. Bazalova M, Nelson G, Noll JM, Graves EE. Modality comparison for small animal radiotherapy: a simulation study. Med Phys 2014; 41: 011710. doi: 10.1118/1.4842415.

7. Shepard D, Ferris M, Olivera G, Mackie T. Optimizing the delivery of radiation therapy to cancer patients. SIAM Rev 1999; 41: 721-44.

8. Otto K. Volumetric modulated arc therapy: IMRT in a single gantry arc. Med Phys 2008; 35 : 310-317. doi: 10.1118/1.2818738.

9. De Boeck L, Beliën J, Egyed W. Dose optimization in high-dose-rate brachytherapy: A literature review of quantitative models from 1990 to 2010. Oper Res Health Care 2013; 3: 80-90.

10. Stewart JMP, Lindsay PE, Jaffray DA. Two-dimensional inverse planning and delivery with a preclinical image guided microirradiator. Med Phys 2013; 40: 101709. doi: 10.1118/1.4819935.

11. Brahme A. Radiation Therapy Physics 1995; 209-246.

12. Niemierko A. Image-guided IMRT 2005; 199-216.

13. Siauw T, Cunha A, Atamtürk A, Hsu I, Pouliot J, Goldberg K. IPIP: a new approach to inverse planning for HDR brachytherapy by directly optimizing dosimetric indices. Med Phys 2011; 38 : 4045-51.

14. Gorissen B, Hoffmann A, Den Hertog D. Mixed integer programming improves comprehensibility and plan quality in inverse optimization of prostate HDR brachytherapy. Phys Med Biol 2013; 58: 1041-57.

15. Pareto V. Manuale di Economica Politica. 1906;

16. Küfer K, Monz M, Scherrer A, Süss P, Alonso F, Sultan A, Bortfeld T, Craft D, Thieke C. Multicriteria optimization in intensity modulated radiotherapy planning. 2005;

17. Craft D, Halabi T, Shih H, Bortfeld T. Approximating convex Pareto surfaces in multiobjective radiotherapy treatment planning. Med Phys 2006; 33: 3399-407.

18. Bokrantz R. Multicriteria optimization for managing tradeoffs in radiation therapy treatment planning. 2013;

19. van Hoof SJ, Granton PV, Verhaegen F. Development and validation of a treatment planning system for small animal radiotherapy: SmART-Plan. Radiother Oncol 2013; 109: 361-366. doi: 
10.1016/j.radonc.2013.10.003.

20. Miettinen K. Nonlinear multiobjective optimization. 1999; 12:

21. Clarkson R, Lindsay PE, Ansell S, Wilson G, Jelveh S, Hill RP, Jaffray DA. Characterization of image quality and image-guidance performance of a preclinical microirradiator. Med Phys 2011; 38: 845-856. doi: 10.1118/1.3533947.

22. Walters B, Kawrakow I, Rogers DWO. DOSXYZnrc Users Manual. 2005; 132. Available from: https://nrc-cnrc.github.io/EGSnrc/doc/pirs794-dosxyznrc.pdf.

23. Granton PV, Podesta M, Landry G, Nijsten S, Bootsma G, Verhaegen F. A combined dose calculation and verification method for a small animal precision irradiator based on onboard imaging. Med Phys 2012; 39: 4155-4166. doi: 10.1118/1.4725710.

24. White DR, Booz J, Griffith RV, Spokas JJ, Wilson IJ. ICRU Report 44, Tissue Substitutes in Radiation Dosimetry and Measurement. J ICRU 1989; os23: NP-NP. doi: 10.1093/jicru/os23.1.Report44.

25. Lee T, Hammad M, Chan T, Sharpe M. Predicting objective function weights from patient anatomy in prostate IMRT treatment planning. Med Phys 2013; 40: 121706.

26. Alfonso JCL, Jagiella N, Núñez L, Herrero MA, Drasdo D. Estimating dose painting effects in radiotherapy: a mathematical model. PLoS ONE 2014; 9: e89380. doi: 10.1371/journal.pone. 0089380 .

27. Holm Å, Larsson T, Tedgren Å. Impact of using linear optimization models in dose planning for HDR brachytherapy. Med Phys 2012; 39: 1021-8. 


\section{Supplementary material}

As an example, we give a step-by-step discussion of the automated optimisation for case 3 , where a crescent-shaped tumour was irradiated using six beams. The goal was to find a treatment plan where $V_{95 \%}$ is at least $68 \%$, while limiting the dose to the OARs as much as possible.

In the first iteration, the weights of the four OARs were all set to 0.25 . The model was solved for a target optimisation weight varying from 0.1 to 0.9 with steps of 0.1 . This resulted in 9 treatment plans with DVH metrics as presented in table 4.3.

Table 4.3: Dose-volume metrics using automatic beam-on time optimisation where the optimisation weights for the OARs are all equal to 0.25 , and the PTV optimisation weights vary from 0.1 to 0.9 with a step size of 0.1 . Dose values are given in Gy, and $V_{95 \%}$ is given as a percentage of the total PTV volume.

\begin{tabular}{lrrrrrrrrr}
\hline$w_{P T V}$ & 0.1 & 0.2 & 0.3 & 0.4 & 0.5 & 0.6 & 0.7 & 0.8 & 0.9 \\
\hline PTV & & & & & & & & & \\
$V_{95 \%}(\%)$ & 55.1 & 69.0 & 73.5 & 73.8 & 73.5 & 88.2 & 90.2 & 90.3 & 90.4 \\
$D_{\min }$ (Gy) & 0 & 0 & 0 & 0 & 0 & 0 & 0 & 0 & 0 \\
$D_{95 \%}$ (Gy) & 0.2 & 0.4 & 0.5 & 0.5 & 0.6 & 2.2 & 2.4 & 2.3 & 2.3 \\
$D_{\operatorname{mean}}$ (Gy) & 8.7 & 8.7 & 10.8 & 10.8 & 10.8 & 10.8 & 10.8 & 10.8 & 10.8 \\
$D_{5 \%}$ (Gy) & 26.2 & 16.2 & 21.7 & 21.0 & 21.9 & 19.6 & 19.4 & 19.6 & 19.6 \\
$D_{\max }$ (Gy) & 29.1 & 26.5 & 33.1 & 33.0 & 29.0 & 21.7 & 22.7 & 22.3 & 22.0 \\
\hline left kidney & & & & & & & & & \\
$D_{5 \%}$ (Gy) & 0.0 & 5.7 & 6.0 & 6.2 & 3.8 & 7.0 & 7.3 & 7.4 & 7.6 \\
$D_{1 \%}$ (Gy) & 0.1 & 6.3 & 6.7 & 6.9 & 4.1 & 7.5 & 7.8 & 7.9 & 8.1 \\
\hline GI & & & & & & & & & \\
$D_{5 \%}$ (Gy) & 0.1 & 0.3 & 0.4 & 0.4 & 2.5 & 4.8 & 5.0 & 5.2 & 5.3 \\
$D_{1 \%}$ (Gy) & 1.3 & 6.2 & 6.7 & 6.8 & 4.3 & 5.7 & 5.9 & 6.0 & 6.2 \\
\hline right kidney & & & & & & & & & \\
$D_{5 \%}$ (Gy) & 0.3 & 0.3 & 0.4 & 0.4 & 0.4 & 6.2 & 6.7 & 6.9 & 7.0 \\
$D_{1 \%}$ (Gy) & 0.4 & 0.4 & 0.5 & 0.5 & 0.5 & 6.5 & 7.1 & 7.3 & 7.4 \\
\hline spine & & & & & & & & & \\
$D_{5 \%}$ (Gy) & 0.8 & 0.7 & 0.9 & 0.9 & 0.9 & 0.9 & 0.9 & 0.9 & 0.9 \\
$D_{1 \%}$ (Gy) & 14.1 & 5.4 & 8.9 & 8.6 & 8.9 & 9.2 & 9.3 & 9.3 & 9.3 \\
\hline
\end{tabular}

The results in table 4.3 show that a PTV weight around 0.2 was suitable to obtain a $V_{95 \%}$ (PTV) of around $68 \%$. Furthermore, $D_{5 \%}$ is higher for the left kidney than for the other OARs. Based on these results, our next step was to optimize treatment plans where the optimization weight is 0.5 for the left kidney, 1/6 for the three remaining OARs, and the PTV weight is varied from 0.15 to 0.25 with a step size of 0.01 . The 
Table 4.4: Dose-volume metrics using automatic beam-on time optimisation where the optimisation weight for the left kidney is 0.5 , the weights for the three remaining OARs are all equal to $1 / 6$, and the PTV optimisation weights vary from 0.15 to 0.25 with a step size of 0.01 . Dose values are given in Gy, and $V_{95}$ is given as a percentage of the total PTV volume.

\begin{tabular}{lrrrrrrrrrrr}
\hline$w_{P T V}$ & 0.15 & 0.16 & 0.17 & 0.18 & 0.19 & 0.20 & 0.21 & 0.22 & 0.23 & 0.24 & 0.25 \\
\hline$P T V$ & & & & & & & & & & & \\
$V_{95 \%}(\%)$ & 54.9 & 54.8 & 54.8 & 54.8 & 54.7 & 54.7 & 56.3 & 56.4 & 65.1 & 68.3 & 69.3 \\
$D_{\min }$ (Gy) & 0 & 0 & 0 & 0 & 0 & 0 & 0 & 0 & 0 & 0 & 0 \\
$D_{95 \%}$ (Gy) & 0.2 & 0.2 & 0.2 & 0.2 & 0.2 & 0.2 & 0.4 & 0.4 & 0.5 & 0.5 & 0.5 \\
$D_{\text {mean }}$ (Gy) & 8.6 & 8.6 & 8.6 & 8.6 & 8.6 & 8.6 & 9.0 & 9.2 & 10.6 & 10.8 & 10.8 \\
$D_{5 \%}$ (Gy) & 26.1 & 26.1 & 26.1 & 26.1 & 26.1 & 26.1 & 21.7 & 21.7 & 23.1 & 23.4 & 23.3 \\
$D_{\text {max }}$ (Gy) & 28.9 & 28.9 & 28.9 & 28.9 & 28.8 & 28.8 & 27.5 & 28.3 & 32.4 & 33.1 & 33.1 \\
\hline left kidney & & & & & & & & & & & \\
$D_{5 \%}$ (Gy) & 0.1 & 0.1 & 0.1 & 0.1 & 0.1 & 0.1 & 3.1 & 3.6 & 5.1 & 5.3 & 5.4 \\
$D_{1 \%}$ (Gy) & 0.1 & 0.1 & 0.1 & 0.1 & 0.1 & 0.1 & 3.5 & 3.9 & 5.6 & 5.8 & 5.9 \\
\hline GI & & & & & & & & & & & \\
$D_{5 \%}$ (Gy) & 0.2 & 0.2 & 0.2 & 0.2 & 0.2 & 0.2 & 0.3 & 0.3 & 0.4 & 0.4 & 0.4 \\
$D_{1 \%}$ (Gy) & 1.3 & 1.3 & 1.3 & 1.3 & 1.3 & 1.3 & 3.6 & 4.1 & 5.7 & 5.9 & 6.0 \\
\hline right kidney & & & & & & & & & & & \\
$D_{5 \%}$ (Gy) & 0.3 & 0.3 & 0.3 & 0.3 & 0.3 & 0.3 & 0.3 & 0.3 & 0.4 & 0.4 & 0.4 \\
$D_{1 \%}$ (Gy) & 0.4 & 0.4 & 0.4 & 0.4 & 0.4 & 0.4 & 0.4 & 0.4 & 0.5 & 0.5 & 0.5 \\
\hline spine & & & & & & & & & & & \\
$D_{5 \%}$ (Gy) & 0.8 & 0.8 & 0.8 & 0.8 & 0.8 & 0.8 & 0.8 & 0.8 & 0.9 & 0.9 & 0.9 \\
$D_{1 \%}$ (Gy) & 13.6 & 13.6 & 13.5 & 13.5 & 13.4 & 13.4 & 9.6 & 9.3 & 9.2 & 9.2 & 9.2 \\
\hline
\end{tabular}

resulting DVH parameters can be found in Table 4.4 .

The DVH metrics after the second iteration show that a $V_{95 \%}$ of at least $68 \%$ cannot be achieved without accepting a relatively high dose to the left kidney. A notable result is the sudden increase in $D_{5 \%}$ for the left kidney when increasing the PTV weight from 0.20 to 0.21 . This is caused by a beam traversing the left kidney that is added to the treatment plan when increasing the PTV weight. Furthermore, $D_{1 \%}$ for the spine increased strongly, which is undesirable since this is a serial organ. Therefore, we reran the previous iteration, but changed the weights for the left kidney and spine to $1 / 6$ and 0.5 , respectively. This yielded the dose-volume metrics as presented in table 4.5 .

From the results in table 4.5, we can conclude that these OAR optimisation weights yield a sufficient sparing of all OARs. The change of OAR weights influenced the dose to the target, and $V_{95 \%}$ increased beyond the desired level. This implied that we can allow for a decrease in target by lowering $w_{P T V}$ in order to achieve a lower dose to the OARs. We re-optimised the models with the weight for the spine set to 0.5 , 
Table 4.5: Dose-volume metrics using automatic beam-on time optimisation where the optimisation weight for the spine is 0.5 , the weights for the three remaining OARs are all equal to $1 / 6$, and the PTV optimisation weights vary from 0.15 to 0.25 with a step size of 0.01 . Dose values are given in Gy, and $V_{95 \%}$ is given as a percentage of the total PTV volume.

\begin{tabular}{lrrrrrrrrrrr}
\hline$w_{P T V}$ & 0.15 & 0.16 & 0.17 & 0.18 & 0.19 & 0.20 & 0.21 & 0.22 & 0.23 & 0.24 & 0.25 \\
\hline$P T V$ & & & & & & & & & & & \\
$V_{95 \%}(\%)$ & 70.0 & 70.1 & 70.3 & 70.4 & 70.5 & 70.6 & 70.7 & 70.8 & 70.9 & 70.9 & 70.7 \\
$D_{\text {min }}$ (Gy) & 0 & 0 & 0 & 0 & 0 & 0 & 0 & 0 & 0 & 0 & 0 \\
$D_{95 \%}$ (Gy) & 0.4 & 0.4 & 0.4 & 0.4 & 0.4 & 0.4 & 0.4 & 0.4 & 0.4 & 0.4 & 0.4 \\
$D_{\text {mean }(\mathrm{Gy})}$ & 8.7 & 8.7 & 8.7 & 8.7 & 8.7 & 8.7 & 8.7 & 8.7 & 8.7 & 8.7 & 8.7 \\
$D_{5 \%}$ (Gy) & 15.1 & 15.1 & 15.0 & 15.0 & 14.9 & 14.9 & 14.9 & 14.9 & 14.9 & 14.6 & 14.6 \\
$D_{\max \text { (Gy) }}$ & 26.2 & 26.2 & 26.2 & 26.2 & 26.2 & 26.2 & 26.2 & 26.2 & 26.2 & 26.2 & 26.2 \\
\hline left kidney & & & & & & & & & & & \\
$D_{5 \%}$ (Gy) & 5.9 & 5.9 & 6.0 & 6.0 & 6.0 & 6.1 & 6.1 & 6.1 & 6.1 & 6.2 & 6.2 \\
$D_{1 \%}$ (Gy) & 6.5 & 6.6 & 6.6 & 6.7 & 6.7 & 6.7 & 6.7 & 6.8 & 6.8 & 6.8 & 6.8 \\
\hline GI & & & & & & & & & & & \\
$D_{5 \%}$ (Gy) & 0.4 & 0.4 & 0.4 & 0.4 & 0.4 & 0.4 & 0.4 & 0.4 & 0.4 & 0.4 & 0.4 \\
$D_{1 \%}$ (Gy) & 6.6 & 6.6 & 6.7 & 6.7 & 6.7 & 6.7 & 6.8 & 6.8 & 6.8 & 6.8 & 6.8 \\
\hline right kidney & & & & & & & & & & & \\
$D_{5 \%}$ (Gy) & 0.4 & 0.4 & 0.4 & 0.4 & 0.4 & 0.4 & 0.4 & 0.4 & 0.4 & 0.4 & 0.4 \\
$D_{1 \%}$ (Gy) & 0.5 & 0.5 & 0.5 & 0.5 & 0.5 & 0.5 & 0.5 & 0.5 & 0.5 & 0.5 & 0.5 \\
\hline spine & & & & & & & & & & & \\
$D_{5 \%}$ (Gy) & 0.6 & 0.6 & 0.6 & 0.6 & 0.6 & 0.6 & 0.6 & 0.6 & 0.6 & 0.6 & 0.6 \\
$D_{1 \%}$ (Gy) & 0.9 & 0.9 & 0.9 & 0.9 & 1.0 & 1.1 & 1.1 & 1.2 & 1.5 & 1.8 & 2.3 \\
\hline
\end{tabular}


Table 4.6: Dose-volume metrics using automatic beam-on time optimisation where the optimisation weight for the left kidney is 0.5 , the wieghts for the three remaining OARs are all equal to $1 / 6$, and the PTV optimisation weights vary from 0.05 to 0.14 with a step size of 0.01 . Dose values are given in Gy, and $V_{95}$ is given as a percentage of the total PTV volume.

\begin{tabular}{lrrrrrrrrrr}
\hline$w_{P T V}$ & 0.05 & 0.06 & 0.07 & 0.08 & 0.09 & 0.10 & 0.11 & 0.12 & 0.13 & 0.14 \\
\hline$P T V$ & & & & & & & & & & \\
$V_{95 \%}(\%)$ & 54.7 & 54.9 & 63.4 & 66.9 & 67.9 & 68.6 & 69.1 & 69.4 & 69.6 & 69.9 \\
$D_{\text {min }}$ (Gy) & 0 & 0 & 0 & 0 & 0 & 0 & 0 & 0 & 0 & 0 \\
$D_{95 \%}$ (Gy) & 0.1 & 0.1 & 0.4 & 0.4 & 0.4 & 0.4 & 0.4 & 0.4 & 0.4 & 0.4 \\
$D_{\text {mean }}$ (Gy) & 8.6 & 8.6 & 8.7 & 8.7 & 8.7 & 8.7 & 8.7 & 8.7 & 8.7 & 8.7 \\
$D_{5 \%}$ (Gy) & 23.4 & 23.4 & 16.1 & 15.7 & 15.6 & 15.5 & 15.4 & 15.3 & 15.2 & 15.2 \\
$D_{\text {max }}$ (Gy) & 26.9 & 26.9 & 26.2 & 26.2 & 26.2 & 26.2 & 26.2 & 26.2 & 26.2 & 26.2 \\
\hline left kidney & & & & & & & & & & \\
$D_{5 \%}$ (Gy) & 0.1 & 0.1 & 5.2 & 5.5 & 5.6 & 5.7 & 5.7 & 5.8 & 5.8 & 5.9 \\
$D_{1 \%}$ (Gy) & 0.1 & 0.1 & 5.8 & 6.1 & 6.2 & 6.3 & 6.3 & 6.4 & 6.5 & 6.5 \\
\hline GI & & & & & & & & & & \\
$D_{5 \%}$ (Gy) & 0.2 & 0.2 & 0.4 & 0.4 & 0.4 & 0.4 & 0.4 & 0.4 & 0.4 & 0.4 \\
$D_{1 \%}$ (Gy) & 1.8 & 1.8 & 6.0 & 6.2 & 6.3 & 6.4 & 6.4 & 6.5 & 6.5 & 6.6 \\
\hline right kidney & & & & & & & & & & \\
$D_{5 \%}$ (Gy) & 0.4 & 0.4 & 0.4 & 0.4 & 0.4 & 0.4 & 0.4 & 0.4 & 0.4 & 0.4 \\
$D_{1 \%}$ (Gy) & 0.6 & 0.6 & 0.6 & 0.6 & 0.6 & 0.6 & 0.6 & 0.6 & 0.6 & 0.5 \\
\hline spine & & & & & & & & & & \\
$D_{5 \%}$ (Gy) & 0.8 & 0.8 & 0.6 & 0.6 & 0.6 & 0.6 & 0.6 & 0.6 & 0.6 & 0.6 \\
$D_{1 \%}$ (Gy) & 8.6 & 8.7 & 1.1 & 1.0 & 1.0 & 1.0 & 1.0 & 1.0 & 0.9 & 0.9 \\
\hline
\end{tabular}

the weights for the remaining three OARs equal to $1 / 6$, and a target weight varying from 0.05 to 0.14 in steps of 0.01 . This resulted in the data presented in table 4.6. The treatment plan with a PTV optimisation weight equal to 0.09 was selected as the optimal one. Again note the strong increase in $D_{5 \%}$ for the left kidney when increasing the PTV weight from 0.06 to 0.07 . As before, this is caused by an additional beam that traverses the left kidney. 
Dose painting by dynamic irradiation delivery on an image guided small animal radiotherapy platform

authors

Stefan J van Hoof, Joana B Verde, Frank Verhaegen

adapted from

The British Journal of Radiology 2019; 92: 20180744 


\section{Abstract}

\section{Objectives}

Using synchronized three-dimensional stage translation and multiangle radiation delivery to improve conformality and homogeneity of radiation delivery to complexly shaped target volumes for precision preclinical radiotherapy.

\section{Methods}

A CT image of a mouse was used to design irradiation plans to target the spinal cord and an orthotopic lung tumour. A dose painting method is proposed that combines heterogeneous two-dimensional area irradiations from multiple beam directions. For each beam directions, a two-dimensional area was defined based on the projection of the target volume. Each area was divided into many single beam Monte Carlo simulations, based on radiochromic film characterization of a $2.4 \mathrm{~mm}$ beam of a commercial precision image guided preclinical irradiation platform. Beam-on time optimisation including all simulated beams from multiple beam directions was used to achieve clinically relevant irradiation objects. Dose painting irradiation plans were compared to irradiation plans using a fixed aperture and rotatable variable aperture collimator.

\section{Results \\ Irradiation plans for the proposed dose painting approach achieved good target coverage, similar dose to avoidance structures in comparison with irradiation using a rotatable vari- able aperture collimator, and considerably less dose to avoidance volumes in comparison with irradiation using a non-rotatable fixed aperture collimator. Required calculations and beam-on times were considerably longer for the dose painting method.}

\section{Conclusions}

It was shown that the proposed dose painting strategy is a valuable extension to increase the versatility of current generation precision preclinical radiotherapy platforms. More conformal and homogeneous dose delivery may be achieved at the cost of increased radiation planning and delivery duration.

\section{Advances in knowledge}

More advanced radiation planning for image guided preclinical radiotherapy platforms can improve target dose conformality and homogeneity with the use of optimised dynamic irradiations with synchronized couch translation. The versatility of these platforms can be increased without hardware modifications. 


\subsection{Introduction}

The advent of image guided preclinical radiation research platforms enables radiobiological studies that make use of more conformal and complex dose distributions, compared to previous-generation experiments. These modern platforms use photon energies and a geometrical millimetre-size scale required for preclinical precision irradiation [1, 2]. The hardware and software of current commercial preclinical research platforms have seen advances towards improving different aspects, including radiation delivery [3] and dose planning [4-7].

Preclinical studies to develop and assess novel treatment strategies with high potential for use in a clinical setting could benefit from more complex preclinical irradiation capabilities [8]. Imaging modalities such as x-ray CT [9] and bioluminescence [10, 11] can provide the basis for developing radiation delivery plans derived from online functional and anatomical imaging. The increased interest and use of orthotopic tumour models instead of subcutaneous tumour models increases the demand for more sophisticated irradiation planning and delivery [12].

The technological development of preclinical image guided radiation research platforms could be divided into 4 categories with different of freedom for radiation delivery as shown in figure 5.1. Current state of the art for precision preclinical irradiation is in the transition from type A to type B. In comparison with a set of fixed aperture size collimators, beam shape modulation could improve conformality of precision irradiation, but is challenging at the required spatial scale of about $1 \mathrm{~mm}$ to several $\mathrm{cm}$. Beam sizes down to $1 \mathrm{~mm}$ or smaller are difficult to model and deliver accurately using fixed aperture beam collimation $[3,13]$. Whereas the clinical approach of using multi-leaf beam collimation is not feasible, iris and jaw collimation techniques have been attempted [14-16]. While these approaches are interesting, they are challenging to implement robustly for mm-sized fields and remain unvalidated in literature and are therefore currently not practically applicable. Other available degrees of freedom could be more promising for implementation with current hardware. For example, modulation of beam energy and current, in combination with stage translation and gantry rotation during irradiation.

The purpose of this study is to demonstrate the feasibility of type $C$ irradiation delivery as shown in figure 5.1. Synchronized three-dimensional stage translation and radiation delivery from multiple radiation directions are used to improve conformality of radiation delivery to a complexly shaped target volume. We developed algorithms to 

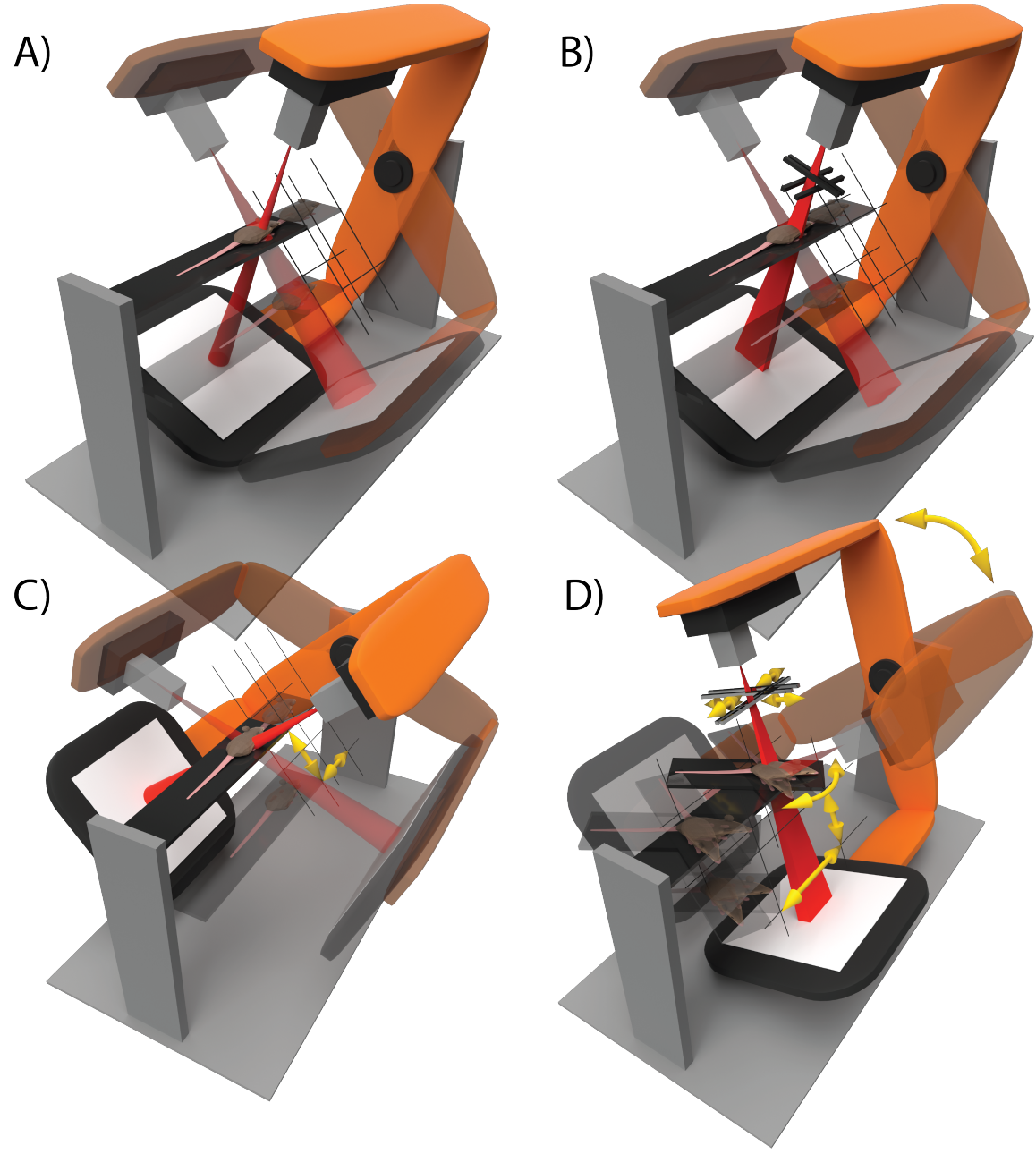

Figure 5.1: The technological development of image guided small animal radiation research platforms can be roughly divided into 4 categories: A) Three-dimensional translation of the animal and gantry rotation are used to perform step-and-shoot wise irradiations in static geometrics. Different beam sizes can be delivered using manually interchangeable collimators. B) Radiation can be delivered using any rectangular beam size with variable aperture collimators. Irradiation delivery remains restricted to step-and-shoot treatments. C) Irregularly shaped, conformal, radiation delivery is enabled by animal translation during irradiation in a dynamic fashion using a fixed beam size. Multiple beam directions can be combined into a single irradiation plan. D) The collimator aperture, gantry angle, animal couch position and angle, and possibly other degrees of freedom such as dose rate, are applied dynamically during irradiation. In all illustrations, semi-transparent elements indicate different states of static step-and-shoot irradiations and yellow arrows indicate movement of the animal couch and beam collimators during radiation delivery. 
create irradiation plans that we simulated using a validated Monte Carlo (MC) model of a preclinical radiotherapy research platform [4]. 


\subsection{Materials and methods}

\subsubsection{Radiation delivery and planning}

A preclinical research platform X-RAD 225Cx (Precision X-Ray, North Branford, CT) was used for CT image guidance and radiation delivery [17]. A $225 \mathrm{kVp} 1.1$ (C1) and 2.4 (C2) $\mathrm{mm}$ diameter beam were used as defined at the isocentre by the full width at half maximum (FWHM). A research version of the dedicated small animal radiotherapy planning system SmART-ATP (version 1.1, SmART Scientific Solutions BV, Maastricht, the Netherlands) was used for radiation planning on a 16 core Intel Core i9-7960X. SmART-ATP uses a validated MC model [4] that was calibrated to the specific dosimetric output of the irradiator. Further irradiation details following the ESTRO ACROP guidelines can be found in supplementary material [18].

\subsubsection{Dynamic irradiation}

SmART-ATP was extended to create the proposed dynamic radiation delivery methods. The X-RAD $225 \mathrm{Cx}$ enables radiation delivery specification using a control point sequence, similar to clinical radiation delivery plans for linear accelerators. The treatment protocol specifies values for all degrees of freedom of the irradiator at specific control points. A treatment protocol specifies one or multiple beams that each consist of at least two control points. Radiation is delivered during beam segments that are defined by two adjacent control points. The delivered dose of a beam segment is specified with cumulative beam-on time. This structure of radiation delivery by control point specification is illustrated in figure 5.2.

By interpolation of the degrees of freedom of the irradiator, a dynamic irradiation can be performed. Currently, the irradiator cannot interpolate specimen stage positions between adjacent control points. Therefore, continuous stage translation was mimicked by using sufficiently small beam segment that use spatially and temporally proximate control points.

To create conformal dynamic irradiation protocols, couch and gantry angle pairs were selected manually. Irradiation protocol creation was divided into multiple two-dimensional area irradiation problems (the red and blue regions in figure 5.2. For each photon incident direction, a couch and gantry angle pair, a beam consisting of many control points is created. The couch rotation angle around a vertical axis is fixed at 0 degrees for the X-RAD 225Cx used in this study. For each beam direction, the delineated target volume is projected onto a virtual plane perpendicular to the 


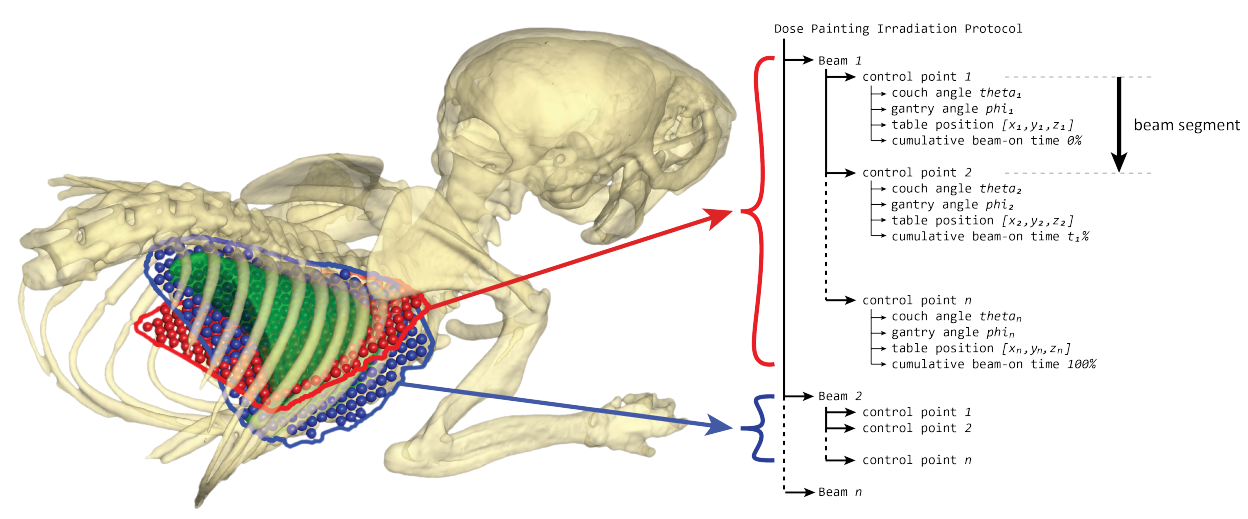

Figure 5.2: An example of a dose painting irradiation protocol structure consisting of two beams at a different gantry angle. The irradiation protocol can consist of multiple beams with each beam consisting of at least two control points. X-rays are turned on at the start of a beam and turned off at the last control point, during which the couch is translated to paint a two-dimensional area. By using beam-on time optimisation and combining multiple radiation directions during optimisation, each two-dimensional area can deliver a heterogeneous dose distribution to the target volume to optimise plan quality.

beam. The projection plane is positioned such that it intersects the centre of mass of the target volume which was calculated assuming a homogeneous target volume density. The two-dimensional target region for the beam direction was calculated as the outer contour of the target volume projection. This outer contour was enlarged with the diameter of the radiation beam to ensure complete target coverage. Absolute positions for the control points were calculated at an equidistance of $0.75 \mathrm{~mm}$, see supplementary material, in a hexagonal grid pattern within the outer contour. Finally, a radiation beam is simulated at each control point. MC simulations for dose painting irradiations were performed to reach $100 \mathrm{k}$ photon histories per square millimetre, to achieve a cumulative statistical uncertainty of at least $3 \%$ for each beam direction. The creation of the simulation grid for a target volume is illustrated in figure 5.3.

\subsubsection{Dose measurement}

Beams were characterized spatially and dosimetrically with radiochromic film positioned at $5 \mathrm{~mm}$ depth between solid water slabs, at the isocentre of the irradiator, and irradiated for 120 seconds. Additional details about the radiochromic film calibration and analysis are provided in supplementary material. Beam dimensions were defined as the FWHM for the lateral and longitudinal direction. Dose rates were defined as the average in an area of $50 \%$ to the maximum dose rate. Beam penumbra sizes were 


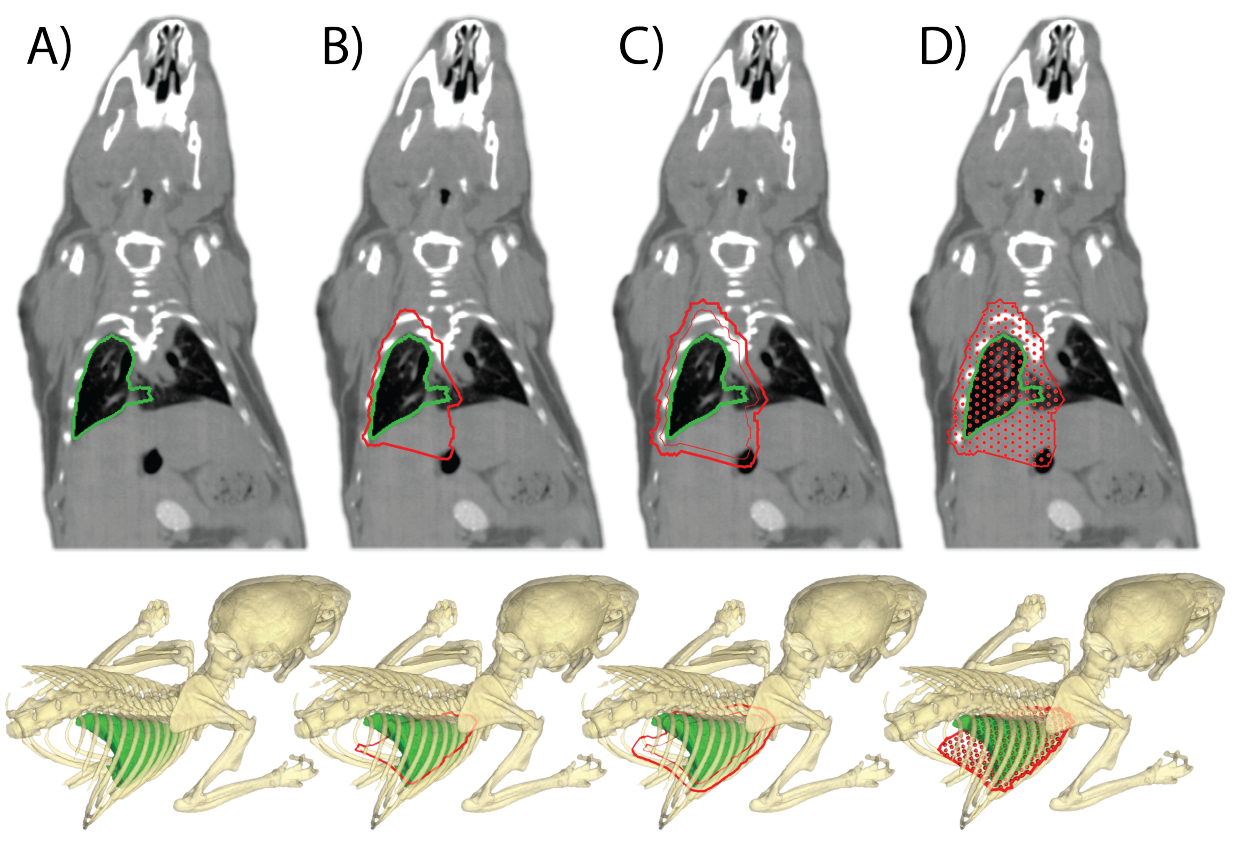

Figure 5.3: The dose painting approach for irradiators of type $C$ as shown in figure 5.1. An example is shown for the calculation of irradiation grid positions to irradiate the whole right lung of a mouse from a single anterior beam direction. A) The target volume delineation to create the irradiation plan. B) A projection outline of the target volume is calculated onto a virtual plane perpendicular to the beam that intersects at the centre of mass of the target volume. C) A margin is added to the projected area to assure target coverage. D) An irradiation grid for the dose calculations is created, which will be used to calculate beam-on times to deliver a specific dose to the target volume at each grid point. Multiple couch and gantry angle pairs can be combined into an irradiation plan. 


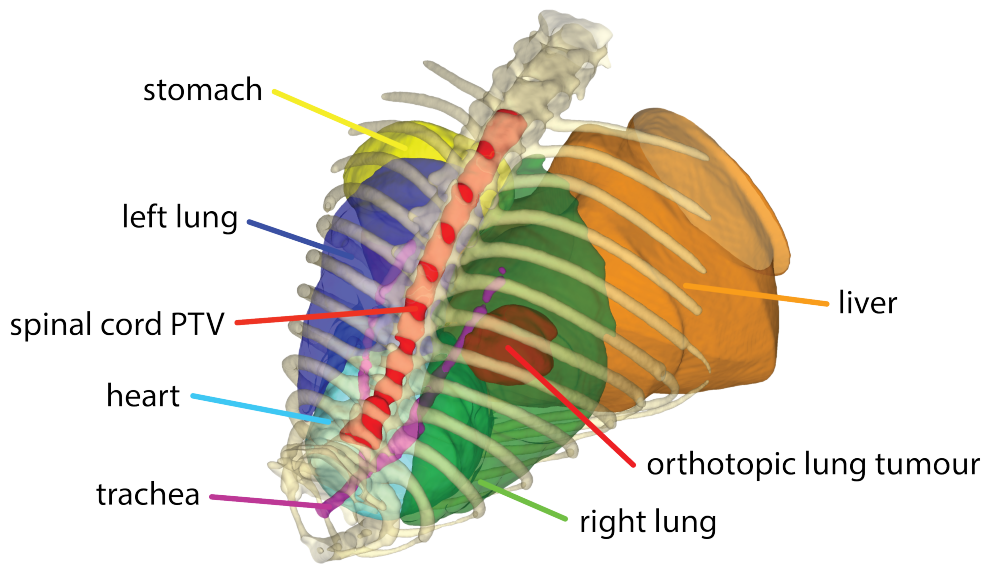

Figure 5.4: An overview of delineated volumes of interest that were considered together with a part of the mouse skeleton for relative spatial reference. Both lungs, the heart, stomach, liver and trachea were included as avoidance regions. The orthotopic lung tumour and a 15 $\mathrm{mm}$ long part of the spinal cord were considered as target volumes for two irradiation cases.

defined as the dose gradient required to cover $20 \%$ to $80 \%$ of the maximum dose.

\subsubsection{Irradiation cases}

Two irradiation scenarios were used based on a cone beam CT image of a mouse with an orthotopic lung tumour. The image was acquired at sufficient resolution and reconstructed to an isotropic voxel spacing of $0.1 \mathrm{~mm}$. A radiation oncologist delineated the orthotopic lung tumour, both lungs, heart, spinal cord, liver, stomach and trachea, see figure 5.4. The orthotopic lung tumour was targeted for case 1 and a length of $15 \mathrm{~mm}$ of the spinal cord was targeted for case 2. Irradiation protocols were created to deliver an arbitrary prescription dose of $10 \mathrm{~Gy}$ to the target volume. No treatment margins were used for sub clinical disease spread or uncertainties in radiation delivery. Therefore, the planning target volume (PTV) was equal to the gross tumour volume (GTV) and dose was planned to the PTV.

Tissue segmentation was performed starting with two Hounsfield Unit (HU) thresholds at -700 and 1200 to segment air, soft tissue, and bone. Thereafter, the left and right lung delineations were used to override the threshold-based segmentation to define lung tissue. For both cases, plans were created for irradiations using a fixed aperture collimator, rotatable variable aperture collimator (RVAC), and for the dose painting approach as described in section 2.2. For case 1, 4 gantry angles were selected 
in the anterior, posterior, lateral and medial direction at 90-degree offsets. For case 2, two parallel opposed lateral gantry angles were selected. For all simulations, an isotropic dose simulation grid resolution of $0.2 \mathrm{~mm}$ was used.

Irradiation plans for the fixed aperture irradiations were created using the smallest beam sizes available for the X-RAD 225Cx which are large enough to achieve complete target coverage. For case 1 this was a circular $5 \mathrm{~mm}$ diameter field and for case 2 this was a square 20 by $20 \mathrm{~mm}$ field, defined as the FWHM at the isocentre. The RVAC plans were created using optimal custom rotation and aperture sizes. Collimator rotations were determined by calculating the angle of the longest direction of the minimum volume enclosing ellipsoid for the target volume, using a solver based on the Khachiyan algorithm [19]. The dose painting irradiation plans were created at hexagonal grid spacings of $0.75 \mathrm{~mm}$, which resulted in a total of 256 beams for case 1 for the 4 gantry angles combined, and a total of 280 beams for case 2 .

\subsubsection{Beam-on time optimisation}

All irradiation plans were required to achieve a $D_{95 \%}$ and $V_{95 \%}$ of $100 \%$. For case 1 , beam-on times for all three irradiation methods were calculated using the beam-on time optimisation framework as described in Balvert et al. [5]. The lung tumour PTV was set as target volume, and the heart, left lung, and spinal cord were considered as avoidance volumes with optimisation weights of $0.4,0.3$ and 0.3 , respectively. The minimum and maximum dose were constrained to $95 \%$ and $120 \%$ of the prescription dose with a minimum mean target dose of 10 Gy as constraint. Multiple optimisation iterations were performed to minimize the dose to avoidance volumes. Optimisation for the dose painting method for case 1 was performed at a downscaled isotropic voxel resolution of $0.5 \mathrm{~mm}$ to speed up optimisation calculations. All beams from the 4 gantry angles were included simultaneously in the optimisation.

For case 2, beam-on times for the fixed aperture and RVAC irradiation plans were linearly scaled to achieve the $D_{95 \%}$ and $V_{95 \%}$ of $100 \%$ with minimal total dose and a mean target dose of at least 5 Gy delivered from each of the two gantry angles. Beamon times for the dose painting method for case 2 were calculated using the beam-on time optimisation framework using the same constraints as for case 1. Beam-on time optimisations for case 2 were performed at the full isotropic dose voxel resolution of $0.2 \mathrm{~mm}$, but for each gantry angle separately such that a minimum mean dose of 5 Gy was delivered from each beam direction. 
When resulting beam-on times of the optimisation framework did not fully satisfy the requirement of a $D_{95 \%}$ of $100 \%$, beam-on times of all beams were increased linearly with the same factor to satisfy that requirement. For each beam 50k photon histories were simulated starting from a phase space file scored at $40 \mathrm{~mm}$ from the isocentre to reach a cumulative statistical uncertainty of less than $3 \%$.

\subsection{Results}

\subsubsection{Beam characterization}

Figure 5.5 shows dose profiles, beam dimensions and beam penumbras extracted from two-dimensional dose images from the radiochromic film measurements of collimator $\mathrm{C} 1$ and $\mathrm{C} 2$. Average beam dimensions were measured to be 1.1 and $2.4 \mathrm{~mm}$ FWHM. Average beam penumbra sizes for the longitudinal and lateral direction were 0.4 and $0.5 \mathrm{~mm}$ for $\mathrm{C} 1$ and $\mathrm{C} 2$, respectively. For $\mathrm{C} 1$, an average dose rate of 0.18 $\mathrm{cGy} / \mathrm{mAs}$ was measured in an area of $0.9 \mathrm{~mm}^{2}$. For C2, an average dose rate of 0.29 $\mathrm{cGy} / \mathrm{mAs}$ was measured in an area of $4.5 \mathrm{~mm} 2$. This makes the irradiated area of C1 $81 \%$ smaller than $\mathrm{C} 2$, with a $38 \%$ lower absolute dose rate. Combined, to paint a dose in the same volume treatment, the irradiation delivery time is 8.4 times higher when using $\mathrm{C} 1$ compared to $\mathrm{C} 2$.

\subsubsection{Irradiation cases}

The duration of the different phases of the radiation planning process and the number of simulated beams are shown in table 5.1. Radiation simulation for the dose painting method for both cases required considerably longer in comparison with the fixed aperture and RVAC methods, with about half of the time spent on data processing. The total CPU time for the simulation of a single beam on a single physical CPU was 4-5 seconds for both cases. The time required to calculate the simulation setup was about 8 seconds for the dose painting method for both cases, and a negligible amount time was needed to calculate the beam size and collimator rotation for the RVAC simulation setups. A few minutes were spent on the complete beam-on time optimisation process, with single optimisation iterations taking less than one minute for all cases. Good target coverage was achieved for all plans with sharp cumulative DVHs for both cases and all irradiation methods, see figure 5.6 and 5.7. The circular $2.4 \mathrm{~mm}$ beam was used for all irradiations using the dose painting method. 

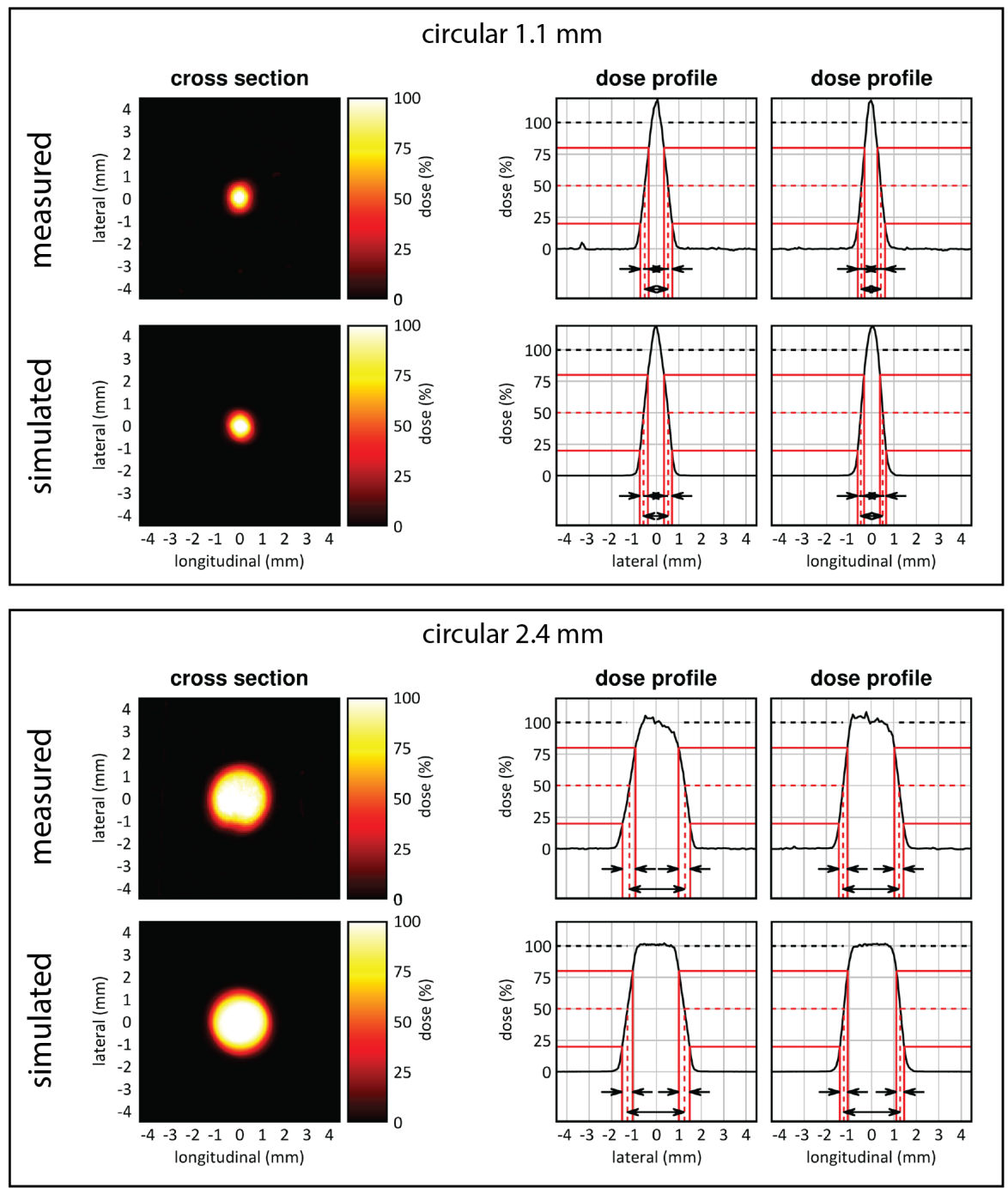

Figure 5.5: Two-dimensional beam characterization of the top) 1.1 and bottom) $2.4 \mathrm{~mm}$ beam using radiochromic film measurements and MC simulations. The irradiated area of the 1.1 $\mathrm{mm}$ beam is $81 \%$ smaller and has a $38 \%$ lower absolute dose rate in comparison with the 2.4 $\mathrm{mm}$ beam. 

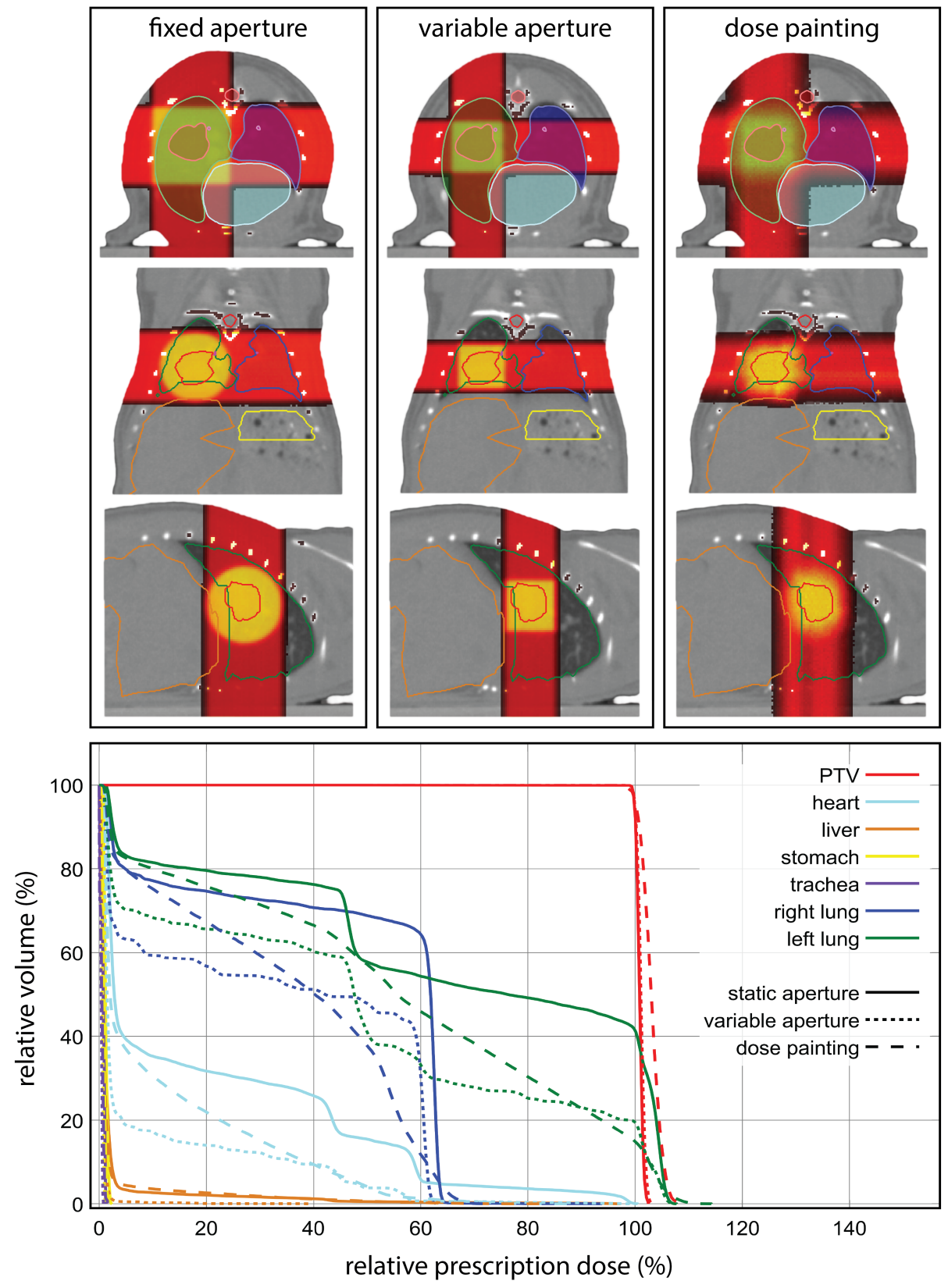

Figure 5.6: At the top an overview of the resulting dose distributions for the fixed aperture, RVAC and dose painting irradiation approaches for the irradiation of an orthotopic tumour in the right lung. Below the resulting cumulative dose volume histograms for all volumes of interest for all three irradiation methods. 

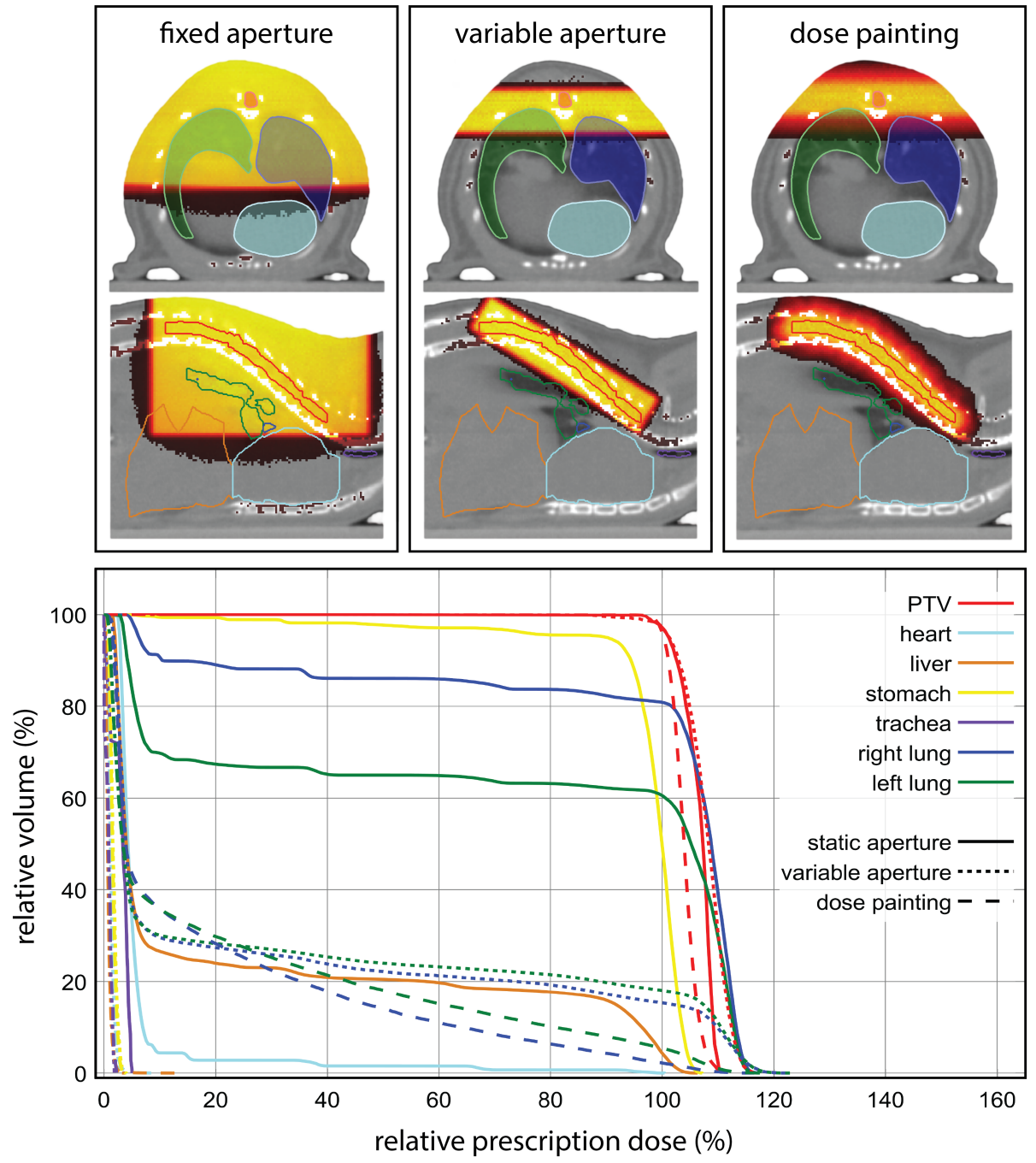

Figure 5.7: At the top an overview of the resulting dose distributions for the fixed aperture, RVAC and dose painting irradiation approaches for the irradiation of a part of the spinal cord from two parallel opposed lateral radiation incident directions. Below the resulting cumulative dose volume histograms for all volumes of interest for all three irradiation methods. 
Table 5.1: The durations of different stages of the radiation delivery and planning process in seconds. Beam-on times were calculated for a beam current of $13 \mathrm{~mA}$. MC simulations were run with parallel threads and performed to reach $100 \mathrm{k}$ photon histories per square millimetre. For the fixed aperture and RVAC methods for case 2, the beam-on times were scaled to achieve a $D_{95 \%}$ and $V_{95 \%}$ of $100 \%$ with minimal total beam-on time, and each beam angle delivering at least 5 Gy to the target volume, without requiring the beam-on time optimisation framework. The durations for the optimisation indicate the time required for a single optimisation iteration. For the complete optimisation of the irradiation plans, a few optimisation iterations were required.

\begin{tabular}{|c|c|c|c|c|c|c|}
\hline & \multicolumn{3}{|c|}{ case 1} & \multicolumn{3}{|c|}{ case 2} \\
\hline & $\begin{array}{c}\text { fixed } \\
\text { aperture }\end{array}$ & $\begin{array}{l}\text { variable } \\
\text { aperture }\end{array}$ & $\begin{array}{c}\text { dose } \\
\text { painting }\end{array}$ & $\begin{array}{c}\text { fixed } \\
\text { aperture }\end{array}$ & $\begin{array}{l}\text { variable } \\
\text { aperture }\end{array}$ & $\begin{array}{c}\text { dose } \\
\text { painting }\end{array}$ \\
\hline number of simulated beams (-) & 4 & 4 & 256 & 2 & 2 & 280 \\
\hline anterior & 1 & 1 & 68 & - & - & - \\
\hline posterior & 1 & 1 & 61 & - & - & - \\
\hline lateral left & 1 & 1 & 66 & 1 & 1 & 140 \\
\hline lateral right & 1 & 1 & 61 & 1 & 1 & 140 \\
\hline total dose calculation (s) & 107 & 64 & 1377 & 160 & 58 & 1556 \\
\hline parallel MC simulations & 73 & 38 & 633 & 132 & 33 & 749 \\
\hline data import & 11 & 11 & 648 & 5 & 5 & 710 \\
\hline data processing & 23 & 15 & 96 & 23 & 20 & 97 \\
\hline single optimisation (s) & 14 & 15 & 59 & - & - & 15 \\
\hline total beam-on time (s) & 224 & 229 & 1587 & 221 & 238 & 1954 \\
\hline anterior & 58 & 64 & 361 & - & - & - \\
\hline posterior & 39 & 38 & 478 & - & - & - \\
\hline lateral left & 79 & 78 & 358 & 111 & 120 & 977 \\
\hline lateral right & 49 & 49 & 378 & 110 & 118 & 977 \\
\hline
\end{tabular}

\section{Orthotopic lung tumour irradiation}

The optimiser excluded 44 beams from the final dose painting plan. The smallest available fixed aperture collimator to achieve complete target coverage was an $8 \mathrm{~mm}$ diameter beam. A 5 by $5 \mathrm{~mm}$ square beam was used for the RVAC method at a collimator rotation of 0 degrees. The increased conformality of the RVAC and dose painting method resulted in considerably lower doses to the left lung, trachea and heart in comparison with the fixed aperture irradiation, see figure 5.6. The dose painting method resulting in a slightly lower dose homogeneity in the PTV.

\section{Spinal cord irradiation}

The optimiser excluded 37 beams from the final dose painting plan. The smallest available fixed aperture collimator to achieve complete target coverage was a 20 by $20 \mathrm{~mm}$ square beam. For the RVAC irradiation, the calculated collimator aperture was 3.5 by $14.8 \mathrm{~mm}$ to achieve a beam size of 4.3 by $17.5 \mathrm{~mm}$ at the isocentre of the irradiator, positioned at a collimator angle of 149 degrees relative to the horizontal 
longitudinal direction of the irradiator (into the gantry). The dose painting method resulting in a slightly better dose homogeneity in the target volume than the fixed aperture and RVAC method, see figure 5.7. The fixed aperture irradiation resulted in considerably higher doses being delivered to all avoidance volumes. The dose painting method achieved the best conformality, with a slightly larger volume of low doses to the left and right lung, but considerably lower volume of higher dose to both lungs.

\subsection{Discussion}

In this study the feasibility of a dose painting strategy that makes use of synchronized stage translation and irradiation was shown, using a dedicated small animal radiation planning system and irradiator. For the irradiation cases discussed, the dose painting method produces irradiation plans with good target volume coverage, similar dose to avoidance volumes in comparison with irradiation using a RVAC at optimal angle and beam aperture, and considerably less dose to avoidance volumes in comparison with irradiation using a non-rotatable fixed aperture collimator.

The dose painting method can achieve superior conformality at the expense of increased radiation planning and delivery duration. With the use of suitable advanced software and user interface, radiation planning for the dose painting method is not considerably more complicated and can be applied using current generation image guided preclinical irradiation platforms. In comparison with the use of a RVAC, the proposed dose painting method including the beam-on time optimisation takes the local tissue environment into account based on MC simulations, to enable fine heterogeneous dose delivery throughout the target volume, either to improve target dose homogeneity, see figure 5.7, or to enable three-dimensional heterogeneous volumetric target dose irradiation when radiation planning software would enable this.

Variable aperture collimators enable the selection of optimal beam sizes and relieves the user from manual collimator placement, which is relatively prone to errors and time consuming as the gantry may need to rotate to a specific angular range to allow collimator exchange. However, the implementation of variable aperture collimators requires thorough validation for mechanical stability and radiation leakage, especially at the small beam sizes used in precision preclinical radiotherapy. To our knowledge, the use of variable aperture collimators for preclinical image guided radiotherapy remains unvalidated in the literature, which makes the proposed dose painting method an excellent candidate to improve plan conformality using current generation hardware. 
The main disadvantage of the dose painting method is the increased radiation delivery duration, see table 5.1, which ultimately concerns the trade-off between larger beam sizes that offer higher dose rates but lower spatial frequencies, and smaller beam sizes that enable smaller dosimetric feature sizes but increase total beam-on time [7]. A side effect of increased irradiation duration is the increased proneness to motion errors. The use of multiple collimator apertures or a RVAC in combination with the proposed dose painting method could improve the overall dose rate while minimizing impact to dose conformality. Preferably, the radiation beam size, and consequently maximum dose rate and achievable dose resolution, should be determined by the radiation planning system based on time and plan quality constraints, rather than by manual selection by the user.

Reducing beam sizes rapidly increases the irradiation duration because besides the smaller irradiated volumes, dose rates decrease rapidly. A reduction from a 2.4 to 1.1 $\mathrm{mm}$ beam, figure 5.5, leads to a more than 8 times higher required beam-on time. It is recognized that margin management for preclinical irradiations differs from clinical practice. For example, there may be no need to account for sub clinical disease spread, but margins for motion and radiation targeting inaccuracies are still required. Considering common imaging resolutions and radiation targeting uncertainties for current generation hardware [1, 3, 17, 20], and initial work on margins [21, 22], a need to use smaller beam sizes than the $2.4 \mathrm{~mm}$ used in this study is not foreseen for the majority or preclinical irradiation studies.

Currently, the irradiator used in this study can be equipped with an x-ray tube rated up to 4500 Watt in combination with a sufficiently capable high voltage generator. This enables a beam current of $20 \mathrm{~mA}$ at $225 \mathrm{kVp}$ instead of $13 \mathrm{~mA}$ used in this study and would decrease reported beam on times by a factor 1.5. Even higher achievable dose rates are not unlikely to become available for these platforms in the future.

The dose painting method currently requires more time for the radiation planning and calculation process. However, the total required number of simulated photon histories is similar to the requirements for a fixed aperture or RVAC simulation, but there is a much higher need for data processing. By relaxing requirements on statistical uncertainty for MC simulations from $3 \%$ used in this study to e.g. $5 \%$, calculation times can be reduced. With tighter process integrations and further software optimisations, data processing performance issues are not expected to withhold practical implementation of this approach.

The work of Stewart et al. (2013) [6] resulted in a semi inverse radiation planning 
process for the same irradiator focusing on achieving the smallest possible features sizes for two-dimensional heterogeneous radiation delivery. Our work focused on slightly larger target volumes with inclusion of multiple beam directions in the irradiation protocol, full three-dimensional local tissue heterogeneities in the MC simulations and optimisation, and the absence of the need to define an a priori number of beams. Similarly, this study also results in a semi inverse radiation planning procedure and could also benefit beam angle selection or optimisation.

In comparison with fixed aperture and RVAC irradiations, a benefit of the dose painting approach is that it can achieve conformal irradiation of concave target volumes. When also including multiple beam directions and beam-on time optimisation, it enables increased versatility to avoid certain avoidance volumes and deliver heterogeneous dose distributions to target volumes.

\subsection{Conclusion}

This study showed that planning and delivery of optimised dynamic irradiation synchronized to couch motion using a precision image guided preclinical radiotherapy platform is a valuable extension for current generation hardware to increase irradiation target dose conformality and homogeneity. These benefits come at the cost of increased radiation planning and delivery duration. For target volumes that closely match available fixed aperture radiation field shapes and sizes, there is limited to no added value. When no fixed aperture collimators closely match the required field size or when more complexly shaped target volumes such as a concave shape, the proposed dose painting may surpass the plan quality achievable with a rotatable variable aperture collimator. The combination of the dose painting approach using multiple beam directions with beam-on time optimisation can also be used to semi-inversely plan and deliver three-dimensional non-uniform dose distributions. Since this irradiation strategy only requires more advanced software and no hardware modifications, there is an overall benefit that increases the versatility of current generation precision image guided irradiation platforms.

\section{Acknowledgments}

The authors thank Paul De Jean, Steve Ansell and William McLaughlin for valuable discussions on the X-RAD 225Cx small animal irradiator and Pilot software. Disclosure SVH and FV are co-founders of SmART Scientific Solutions BV. and collaborate with Precision X-Ray Inc. 


\section{References}

1. Verhaegen F, Granton P, Tryggestad E. Small animal radiotherapy research platforms. Phys. Med. Biol. 2011; 56: R55-83. doi: 10.1088/0031-9155/56/12/R01.

2. Verhaegen F, van Hoof S, Granton PV, Trani D. A review of treatment planning for precision image guided photon beam pre-clinical animal radiation studies. Z. Med. Phys. 2014; 24: 323-334. doi: 10.1016/j.zemedi.2014.02.004.

3. Stewart JMP, Ansell S, Lindsay PE, Jaffray DA. Online virtual isocenter based radiation field targeting for high performance small animal microirradiation. Phys. Med. Biol. 2015; 60: 9031-9046. doi: 10.1088/0031-9155/60/23/9031.

4. van Hoof SJ, Granton PV, Verhaegen F. Development and validation of a treatment planning system for small animal radiotherapy: SmART-Plan. Radiother. Oncol. J. Eur. Soc. Ther. Radiol. Oncol. 2013; 109: 361-366. doi: 10.1016/j.radonc.2013.10.003.

5. Balvert M, van Hoof SJ, Granton PV, Trani D, den Hertog D, Hoffmann AL, Verhaegen F. A framework for inverse planning of beam-on times for $3 \mathrm{D}$ small animal radiotherapy using interactive multi-objective optimisation. Phys. Med. Biol. 2015; 60: 5681-5698. doi: 10.1088/00319155/60/14/5681.

6. Stewart JMP, Lindsay PE, Jaffray DA. Two-dimensional inverse planning and delivery with a preclinical image guided microirradiator. Med. Phys. 2013; 40: 101709. doi: 10.1118/1.4819935.

7. Stewart JMP, Stapleton S, Chaudary N, Lindsay PE, Jaffray DA. Spatial frequency performance limitations of radiation dose optimization and beam positioning. Phys. Med. Biol. 2018; 63: 125006. doi: 10.1088/1361-6560/aac501.

8. Trani D, Yaromina A, Dubois L, Granzier M, Peeters SGJA, Biemans R, Nalbantov G, Lieuwes N, Reniers B, Troost EEGC, Verhaegen F, Lambin P. Preclinical Assessment of Efficacy of Radiation Dose Painting Based on Intratumoral FDG-PET Uptake. Clin. Cancer Res. Off. J. Am. Assoc. Cancer Res. 2015; 21: 5511-5518. doi: 10.1158/1078-0432.CCR-15-0290.

9. Schyns LEJR, Almeida IP, van Hoof SJ, Descamps B, Vanhove C, Landry G, Granton PV, Verhaegen $\mathrm{F}$. Optimizing dual energy cone beam CT protocols for preclinical imaging and radiation research. Br. J. Radiol. 2017; 90: 20160480. doi: 10.1259/bjr.20160480.

10. Weersink RA, Ansell S, Wang A, Wilson G, Shah D, Lindsay PE, Jaffray DA. Integration of optical imaging with a small animal irradiator. Med. Phys. 2014; 41: 102701. doi: 10.1118/1.4894730.

11. Zhang B, Wang KK-H, Yu J, Eslami S, lordachita I, Reyes J, Malek R, Tran PT, Patterson MS, Wong JW. Bioluminescence Tomography-Guided Radiation Therapy for Preclinical Research. Int. J. Radiat. Oncol. Biol. Phys. 2016; 94: 1144-1153. doi: 10.1016/j.jijobp.2015.11.039.

12. Tillner $F$, Thute $P$, Bütof $R$, Krause $M$, Enghardt $W$. Pre-clinical research in small animals using radiotherapy technology-a bidirectional translational approach. Z. Med. Phys. 2014; 24: 335-351. doi: 10.1016/j.zemedi.2014.07.004.

13. Granton PV, Podesta M, Landry G, Nijsten S, Bootsma G, Verhaegen F. A combined dose calculation and verification method for a small animal precision irradiator based on onboard imaging. Med. Phys. 2012; 39: 4155-4166. doi: 10.1118/1.4725710.

14. Graves EE, Zhou H, Chatterjee R, Keall PJ, Gambhir SS, Contag CH, Boyer AL. Design and evaluation of a variable aperture collimator for conformal radiotherapy of small animals using a microCT scanner. Med. Phys. 2007; 34: 4359-4367. doi: 10.1118/1.2789498.

15. Zhou H, Rodriguez M, van den Haak F, Nelson G, Jogani R, Xu J, Zhu X, Xian Y, Tran PT, Felsher DW, Keall PJ, Graves EE. Development of a micro-computed tomography-based 
image-guided conformal radiotherapy system for small animals. Int. J. Radiat. Oncol. Biol. Phys. 2010; 78: 297-305. doi: 10.1016/j.jijrobp.2009.11.008.

16. Woods K, Nguyen D, O'Connor D, Sheng K. IMRT using Double-Focused Sparse Orthogonal Collimators. 2018;

17. Clarkson R, Lindsay PE, Ansell S, Wilson G, Jelveh S, Hill RP, Jaffray DA. Characterization of image quality and image-guidance performance of a preclinical microirradiator. Med. Phys. 2011; 38: 845-856. doi: 10.1118/1.3533947.

18. Verhaegen F, Dubois L, Gianolini S, Hill MA, Karger CP, Lauber K, Prise KM, Sarrut D, Thorwarth D, Vanhove C, Vojnovic B, Weersink R, Wilkens JJ, Georg D. ESTRO ACROP: Technology for precision small animal radiotherapy research: Optimal use and challenges. Radiother. Oncol. J. Eur. Soc. Ther. Radiol. Oncol. 2018; 126: 471-478. doi: 10.1016/j.radonc.2017.11.016

19. Khachiyan LG. Rounding of Polytopes in the Real Number Model of Computation. Math. Oper. Res. 1996; 21: 307-320. doi: 10.1287/moor.21.2.307.

20. Wong J, Armour E, Kazanzides P, lordachita I, Tryggestad E, Deng H, Matinfar M, Kennedy C, Liu Z, Chan T, Gray O, Verhaegen F, McNutt T, Ford E, DeWeese TL. High-resolution, small animal radiation research platform with $\mathrm{x}$-ray tomographic guidance capabilities. Int. J. Radiat. Oncol. Biol. Phys. 2008; 71: 1591-1599. doi: 10.1016/j.jijobp.2008.04.025.

21. Vaniqui A, van der Heyden B, Almeida IP, Schyns LE, van Hoof SJ, Verhaegen F. On the determination of planning target margins due to motion for mice lung tumours using a fourdimensional MOBY phantom. Br. J. Radiol. 2018; 20180445. doi: 10.1259/bjr.20180445.

22. van der Heyden B, van Hoof SJ, Schyns LEJR, Verhaegen F. The influence of respiratory motion on dose delivery in a mouse lung tumour irradiation using the 4D MOBY phantom. Br. J. Radiol. 2017; 90: 20160419. doi: 10.1259/bjr.20160419.

23. Ma CM, Coffey CW, DeWerd LA, Liu C, Nath R, Seltzer SM, Seuntjens JP, American Association of Physicists in Medicine. AAPM protocol for 40-300 kV x-ray beam dosimetry in radiotherapy and radiobiology. Med. Phys. 2001; 28: 868-893. doi: 10.1118/1.1374247.

24. Niroomand-Rad A, Blackwell CR, Coursey BM, Gall KP, Galvin JM, McLaughlin WL, Meigooni AS, Nath R, Rodgers JE, Soares CG. Radiochromic film dosimetry: recommendations of AAPM Radiation Therapy Committee Task Group 55. American Association of Physicists in Medicine. Med. Phys. 1998; 25: 2093-2115. doi: 10.1118/1.598407.

25. Micke A, Lewis DF, Yu X. Multichannel film dosimetry with nonuniformity correction. Med. Phys. 2011; 38: 2523-2534. doi: 10.1118/1.3576105.

26. Van Hoof SJ, Granton PV, Landry G, Podesta M, Verhaegen F. Evaluation of a novel triplechannel radiochromic film analysis procedure using EBT2. Phys. Med. Biol. 2012; 57: 4353-4368. doi: 10.1088/0031-9155/57/13/4353. 


\section{Supplementary material}

\section{ESTRO ACROP parameters}

The orthotopic lung tumour of case 1 had a volume of $30 \mathrm{~mm} 3$ and the targeted part of the spinal cord had a volume of $19 \mathrm{~mm}$. The cone beam CT of the mouse was acquired with the animal in feet first prone position under anaesthesia using isoflurane supported by carbon fibre animal couch. Absolute dosimetry for the irradiator is performed at least every 3 months using a 40 by $40 \mathrm{~mm}$ reference beam following the AAPM TG-61 protocol using the in-air method [1]. Photon beams of $225 \mathrm{kVp}$ with an inherent filtration of $0.8 \mathrm{~mm}$ beryllium and additional filtration of $0.3 \mathrm{~mm}$ copper were used, resulting in a photon spectrum with a first and second half value layer of 0.9 and $1.8 \mathrm{~mm} \mathrm{Cu}$. This provided a dose rate of $0.47+/-0.02 \mathrm{cGy} / \mathrm{mAs}$ at the surface of water at the nominal isocentre of the machine at $304 \mathrm{~mm}$ from the $\mathrm{x}$-ray focal spot for the 4 by $4 \mathrm{~cm}$ reference beam. The imaging and irradiation hardware and capabilities of the irradiation have been described in detail before [2]. The cone beam CT image used in this study was acquired at $80 \mathrm{kVp}, 2.5 \mathrm{~mA}$, filtered by $2 \mathrm{~mm} \mathrm{Al}$, at an acquisition rate of 5 frames/second and gantry revolution of 1 revolution/minute. SmARTATP was used to simulate dose distribution scored as dose-to-medium-in-medium. SmART-ATP used a piecewise linear CT-to-density calibration for the specific imaging protocol used, that was established using a $\mu \mathrm{CT}$ imaging calibration phantom (SmART Scientific Solutions B.V., Maastricht, the Netherlands) with different tissue substitute inserts.

\section{Control point spacing}

To minimize computing time, spacing between grid points for MC calculations should be chosen as large as possible, without deteriorating dose homogeneity, and as large as the required feature sizes of volumes of interest allow. Simulations for the $2.4 \mathrm{~mm}$ beam were performed in a $10 \mathrm{~mm}$ thick virtual water phantom, with beam isocentres positioned at $5 \mathrm{~mm}$ depth, in a hexagonal grid with grid spacings of $0.50,0.75$ and $1.00 \mathrm{~mm}$. MC Simulations were performed to reach a statistical uncertainty of $<1 \%$ in the central region of the beam for each individual beam. Measurements were performed using radiochromic film positioned at $5 \mathrm{~mm}$ depth between solid water slabs. Beam isocentres for the measurements were positioned in a hexagonal grid with grid spacings of $0.50,1.00$ and $1.50 \mathrm{~mm}$ for the $2.4 \mathrm{~mm}$ beam. Irradiations were performed by defining irradiation protocols with control points at all grid positions. Resulting twodimensional dose distributions from the simulations and measurements are shown in figure 5.8 .

\section{Radiochromic film calibration}



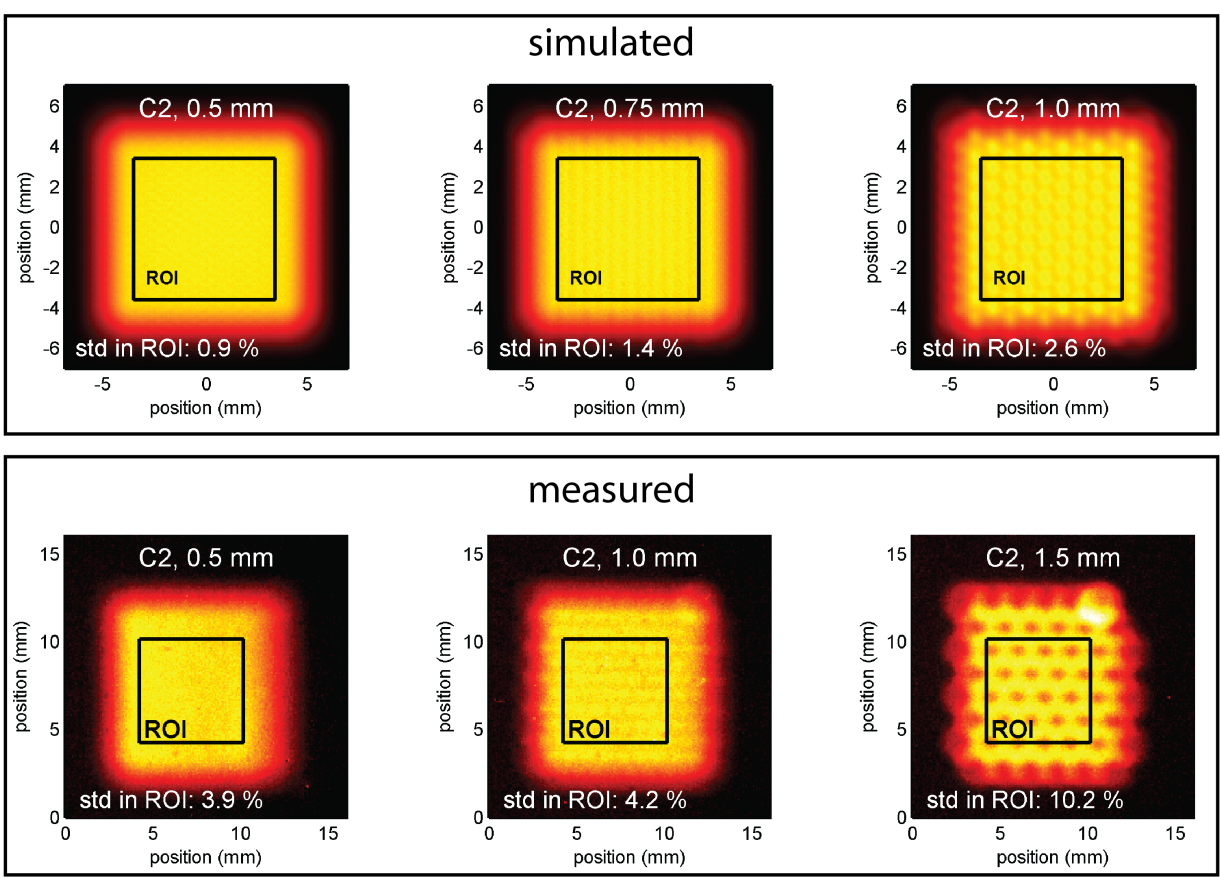

Figure 5.8: Dose homogeneity in two-dimensional areas for A) MC simulations and B) measured with radiochromic film for the $2.4 \mathrm{~mm}$ circular beam. Standard deviations are calculated of the square regions of interest outlined in black. 
Radiochromic film calibration was performed using the same X-RAD 225Cx irradiator that was used for this study. The irradiator was calibrated using an ionization chamber and electrometer traceable to a national standards laboratory. The radiochromic film batch (EBT3, Ashland Inc., Covington, KY) was calibrated by exposing separate film pieces to 7 different known dose levels, using 3 film pieces for each dose level. Film pieces were handled following the recommendations outlined in AAPM TG-55 report [3], and scanned in 48 bit colour transmission mode using an Epson PRO V750 (Seiko Epson Corporation, Nagano, Japan) at $300 \mathrm{dpi}$, with all colour corrections turned off. To convert raw image data to dose, the multichannel film analysis method was applied using three colour channels, using in-house developed and validated software [4, 5]. A consistent protocol was used for film calibration and all measurements, using a post-irradiation read out time of 24 hours after irradiation. Film pieces were scanned using a consistent position and orientation on the scanner.

\section{References supplementary material}

1. Ma CM, Coffey CW, DeWerd LA, Liu C, Nath R, Seltzer SM, Seuntjens JP, American Association of Physicists in Medicine. AAPM protocol for 40-300 kV x-ray beam dosimetry in radiotherapy and radiobiology. Med. Phys. 2001; 28: 868-893. doi: 10.1118/1.1374247.

2. Clarkson R, Lindsay PE, Ansell S, Wilson G, Jelveh S, Hill RP, Jaffray DA. Characterization of image quality and image-guidance performance of a preclinical microirradiator. Med. Phys. 2011; 38: 845-856. doi: 10.1118/1.3533947.

3. Niroomand-Rad A, Blackwell CR, Coursey BM, Gall KP, Galvin JM, McLaughlin WL, Meigooni AS, Nath R, Rodgers JE, Soares CG. Radiochromic film dosimetry: recommendations of AAPM Radiation Therapy Committee Task Group 55. American Association of Physicists in Medicine. Med. Phys. 1998; 25: 2093-2115. doi: 10.1118/1.598407.

4. Micke A, Lewis DF, Yu X. Multichannel film dosimetry with nonuniformity correction. Med. Phys. 2011; 38: 2523-2534. doi: 10.1118/1.3576105.

5. Van Hoof SJ, Granton PV, Landry G, Podesta M, Verhaegen F. Evaluation of a novel triplechannel radiochromic film analysis procedure using EBT2. Phys. Med. Biol. 2012; 57: 4353-4368. doi: 10.1088/0031-9155/57/13/4353. 



\section{An image guided Small Animal Radiation Therapy Platform (SmART) to monitor glioblastoma progression and therapy response}

Sanaz Yahyanejad*, Stefan J van Hoof*, Jan Theys*, Lydie MO Barbeau, Patrick V Granton, Kim Paesmans, Frank Verhaegen, Marc Vooijs adapted from Radiotherapy \& Oncology 2015; 116: 467-472

* contributed equally 


\section{Abstract}

\section{Background and purpose}

Glioblastoma multiforme is the most common malignant brain tumour. Standard treatment including surgery, radiotherapy and chemotherapy with temozolomide is not curative. There is a great need for in vitro and in vivo models closely mimicking clinical practice to ensure better translation of novel preclinical findings.

\section{Methods and materials}

A 3D spheroid model was established using the U87MG cell line. The efficacy of temozolomide, RT and combinations was assessed using growth delay assays. Orthotopic glioblastoma tumours were established, different radiation doses delivered based on micro-CT based treatment planning (SmART-Plan) and dose volume histograms (DVH) were determined. Tumour growth was monitored using bioluminescent imaging.

\section{Results}

3D spheroid cultures showed a dose-dependent growth delay upon single and combination treatments. Precise uniform radiation was achieved in all in vivo treatment groups at all doses tested, and DVHs showed accurate dose coverage in the planning target volume which resulted in tumour growth delay.

\section{Conclusion}

We demonstrate that 3D spheroid technology can be reliably used for treatment efficacy evaluation and that mimicking a clinical setting is also possible in small animals. Both these in vitro and in vivo techniques can be combined for clinically relevant testing of novel drugs combined with radiation. 


\subsection{Introduction}

Glioblastoma multiforme (GBM) is the most common malignant brain tumour in adults. The current standard of care includes surgery followed by radiotherapy (RT) and chemotherapy using temozolomide (TMZ) with median survival after diagnosis of 15 months [1]. TMZ acts via alkylation of $\mathrm{O}^{6}$-Guanine, which induces DNA damage and cell death. However, many patients are observed to acquire TMZ resistance in recurrent gliomas [2]. Therefore, there is an urgent need for reliable in vitro and preclinical models that more closely model the course of human GBM and enable testing of new interventions that improve clinical management and are predictive for disease outcome in patients.

Increasing evidence shows that some drugs that fail to show efficacy in 2D are effective in 3D culture systems [3]. This is significant because spheroids better recapitulate features of solid tumour growth such as inward proliferation and spatiotemporal changes in oxygen concentration reminiscent of tumour hypoxia [4]. Radiation sensitivity is also known to be different between cells grown as monolayer or cells grown in 3D [5]. Therefore, spheroids may be a more relevant in vitro model system to perform drug screening in combination with radiotherapy.

Through these 3D spheroid model systems, once candidate drugs have been identified, preclinical animal models can improve their translational relevance e.g. by reflecting the tumour micro-environment, especially when the clinical treatment practice can be mimicked by use of orthotopic tumour models and precision image guided radiotherapy, downscaled from human radiotherapy conditions. Orthotopic mouse models require non-invasive imaging techniques to monitor disease progression and therapy response longitudinally. Bioluminescence imaging (BLI) is a fast and sensitive method to monitor tumour growth kinetics in vivo. Recently, dedicated image guided small animal radiotherapy (SmART) devices have become available for preclinical research, which combine micro-CT imaging or other modalities (e.g. BLI) with advanced precision irradiation using millimetre sized beams in the same frame of reference making tumour delineation and treatment planning highly accurate thereby limiting normal tissue toxicity [6]. Previously, we have shown that combining BLI with contrast-enhanced micro-CT provides an accurate and sensitive integrated approach to determine in situ tumour volumes and monitoring of disease progression in an orthotopic model of GBM [7]. The focus of this study was to develop a clinically relevant integrated approach using a spheroid drug screening platform in vitro and to demonstrate the power of using high-precision SmART technology in vivo to assess the effect of radiation treatment in a model of intracranial GBM. 


\subsection{Materials and methods}

\subsubsection{Cells and chemical treatments}

U87MG-Luc2 and U87MG-R (resistant cells to TMZ) cells were grown in DMEM (Invitrogen) supplemented with $10 \%$ FBS (Hyclone). U87MG-Luc2 is a widely used glioma cell line transduced with a lentiviral vector expressing firefly luciferase (Perkin Elmer, Waltham, MA). TMZ resistant U87MG-R were generated essentially as described elsewhere [8]. Cell lines were treated with increasing concentration of $T M Z$ (Selleck) (1, 5 and $25 \mathrm{IM}$ ) and/or Cisplatin (Selleck) (2.5 IM). DMSO was used as vehicle for both drugs. TMZ and Cisplatin were stored at $100 \mathrm{mM}$ stock concentration in -80 and room temperature, respectively.

\subsubsection{D spheroid assay}

$0.750 \mathrm{gr}$ agarose (Sigma-Aldrich) was dissolved in $50 \mathrm{ml}$ serum free medium and autoclaved. $50 \mu \mathrm{l}$ of agarose mixture was pipetted per well into the 96-well plate. 2500 cells $/ 200 \mu \mathrm{l}$ of growth medium were seeded on the agarose coated plates per well. After 4 days, one spheroid per well was formed with a diameter of $\sim 400 \mu \mathrm{m}$ (12 spheroids/treatment condition), treatment was started and phase-contrast pictures were taken from individual spheroids $3 x /$ week. Spheroid volumes were analyzed using MATLAB (The MathWorks Inc., Natrick, Ma). Growth medium with drugs was refreshed every 3 days.

\subsubsection{Animal surgery and imaging}

Immunocompromised CD1 nu/nu mice $(n=23)$ were used in this study. Animal work was performed in accordance with national code for the experimentation with animals. Surgical procedures and imaging techniques were performed as described previously [7]. All mice were anesthetized with a gas mixture of air and $4 \%$ isoflurane prior to intracranial surgery. A midline scalp incision was made, the bregma was identified and the guide screw entry was then marked at $3 \mathrm{~mm}$ posterior and $2 \mathrm{~mm}$ right lateral to the bregma and from the cortical surface a $3 \mathrm{~mm}$ deep craniotomy was created. 5 $\mu \mathrm{l}$ cell suspension in PBS $(100,000$ cells) was injected into the brain using a Hamilton syringe. The needle was slowly removed $5 \mathrm{~min}$ after the injection to prevent any reflux of the cells. The skin was closed using skin glue. BLI and contrast-enhanced micro-CT imaging were used to confirm tumour establishment. BLI was performed 3x/week using the Optix MX2 system (ART Inc., Saint-Laurent, QC). Fifteen minutes 
prior to BLI imaging, $150 \mathrm{mg} / \mathrm{kg}$ of d-luciferin (PerkinElmer, Waltham, MA) in PBS was injected intraperitoneally. Images were analyzed using Optix (OptiView version 2.01, ART Inc.). Micro-CT imaging was performed periodically using the small animal microIR (X-RAD 225Cx, Precision X-ray Inc., North Branford, CT). To enhance soft tissue contrast $150 \mu \mathrm{L}$ of the iodinated Omnipaque 350 (GE Healthcare, Little Chalfont, UK) was injected undiluted via the tail vein immediately prior to micro-CT imaging.

\subsubsection{In vivo irradiation}

The dedicated small animal radiotherapy planning system SmART-Plan (version 1.3.1, Precision X-ray, North Branford, CT) was used to create, evaluate, and deliver irradiation [9]. The tumour was delineated as gross target volume (GTV) and the normal brain was delineated as organ at risk (OAR). The planning target volume (PTV) was equal to the GTV and dose was planned to the PTV. Two static parallel opposed beams linked to the irradiator isocentre were used to deliver a homogeneous dose to the PTV. Radiation was delivered using a photon spectrum of $225 \mathrm{kVp}$ at $12 \mathrm{~mA}$, which provides a dose rate of approximately $3 \mathrm{~Gy} /$ minute.

\subsubsection{Statistical analysis}

Statistical analysis was carried out using GraphPad Prism version 5.01. A nonparametric Mann Whitney and one way ANOVA was performed to determine differences in growth delay in vitro and in vivo, respectively. Asterisk indicates significance $\left({ }^{*} p<0.05,{ }^{* *} p<0.01\right.$ and $\left.{ }^{* * *} p<0.001\right)$, ns: not significant. 
A)

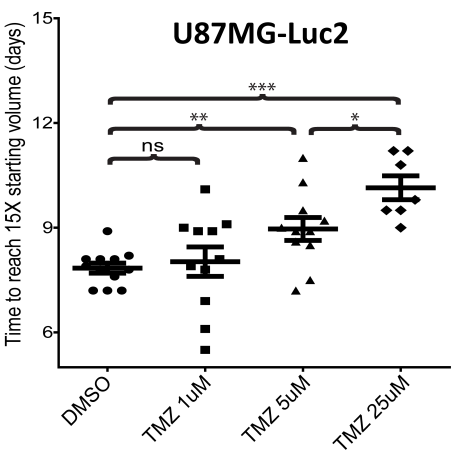

C)

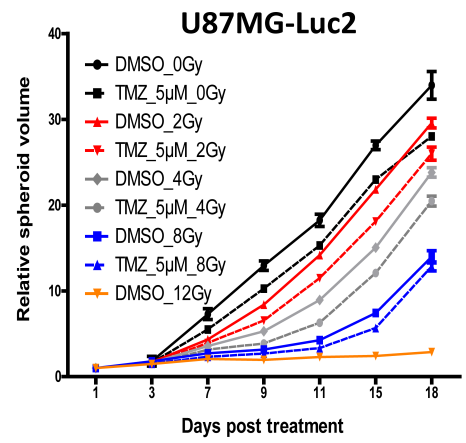

B)

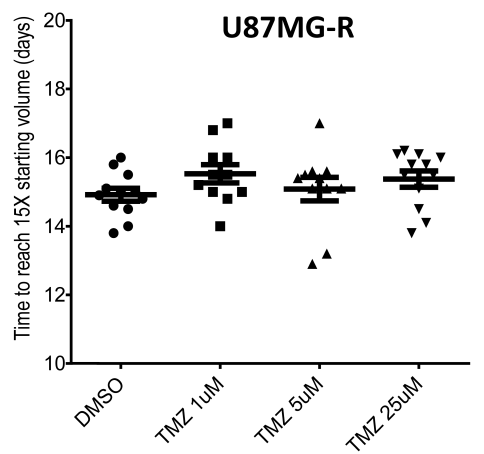

D)

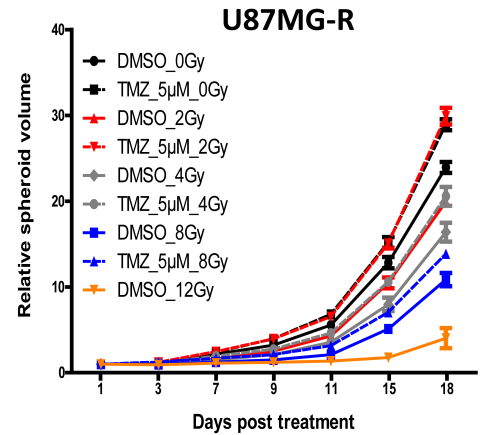

E)

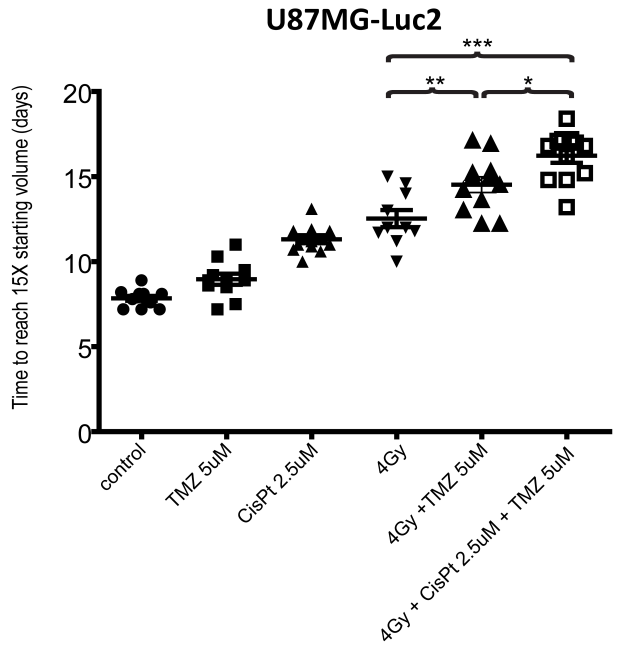

Figure 6.1: Dose-response spheroid growth delay upon treatment with TMZ, RT and their combinations. A) and B) Increasing concentration of TMZ shows an enhanced growth delay for U87MG-Luc2, while no effect is observed in U87MG-R. C) RT and RT+TMZ treatment shows an increased growth delay for U87MG-Luc2. D) RT treatment shows an increased growth delay while $R T+T M Z$ shows to reduce the delay. E) RT+CisPt+TMZ shows an enhanced growth delay compared with RT and RT+TMZ. Spheroid growth delay is expressed in T15SV. 


\subsection{Results}

We established robust spheroid culturing conditions for U87MG-Luc2 cells that yield highly reproducible sphere growth reliable for quantitative treatment efficacy evaluation. Spheres could be maintained in culture for as long as 25 days and reaching up to at least 20 times of their initial treatment starting volume. We first determined the sensitivity of U87MG-Luc2 spheroids to different concentrations of TMZ. Use of increasing concentrations of $\mathrm{TMZ}(1,5$ and $25 \mu \mathrm{M})$ resulted in a significant increase in spheroid growth delay expressed as time to reach 15 times starting volume (T15SV) from 8 days for vehicle to $8.1,8.8$ and 9.8 days, respectively (figure 6.1A). Such $T M Z$ dose-dependent response was not observed in U87MG-R cells (figure 6.1B). We chose $5 \mu \mathrm{M} \mathrm{TMZ}$ as a sub-curative dose for use in combination treatments. In U87MG-Luc2 cells single radiation doses of 2, 4, 8 and 12 Gy resulted in a dosedependent decrease in T15SV from 4.3 days in the non-irradiated group to 5.0 and 5.9 days after 2 and 4 Gy, respectively. Doses of 8 and 12 Gy resulted in such a severe growth delay that $15 \mathrm{SV}$ could not be reached within the course of the experiment. Although the growth rate of the U87MG-R was slower, the response to RT was similar, in that there was an increasing growth delay after 2, 4, 8 and 12 Gy irradiation (figure 6.1D). We then tested the combination treatments and found that addition of TMZ $(5 \mu \mathrm{M})$ resulted in additive effects at every radiation dose level in the U87MGLuc2 cells (figure 6.1C), but not in the U87MG-R cells. If anything, addition of TMZ seemed to make the cells even slightly more radiation resistant (figure 6.1D). These data show the potential of using 3D spheroid technology to reliably quantify treatment efficacy. Exemplified here using the standard treatment of care for GBM (radiation $+T M Z$ ), this methodology can now be applied to test virtually any treatment combination. One of such uncommon, but potentially interesting combination is addition of alkylating agents such as Cisplatin to the standard of care schedule. The addition of Cisplatin to the standard treatment combination in U87MG-Luc2 cells resulted in a significant $(p<0.05)$ increase in spheroid growth delay (figure 6.1E).

To forward novel treatment combinations into patients it is of vital importance not only to have a robust in vitro system for efficacy testing, but also to develop clinically relevant in vivo models and technology. To do so, we applied stereotactic implantation to intracranially inject U87MG-Luc2 cells. BLI measurements (3x/week) were used to monitor tumour growth. Having established the in vivo growth characteristics, we used SmART technology for precise irradiation. Prior to irradiation, tumours were delineated using contrast-enhanced micro-CT for planning the radiation delivery (figure 6.2B). Upon 3 consecutive increases of BLI signal, indicative of active tumour growth, 

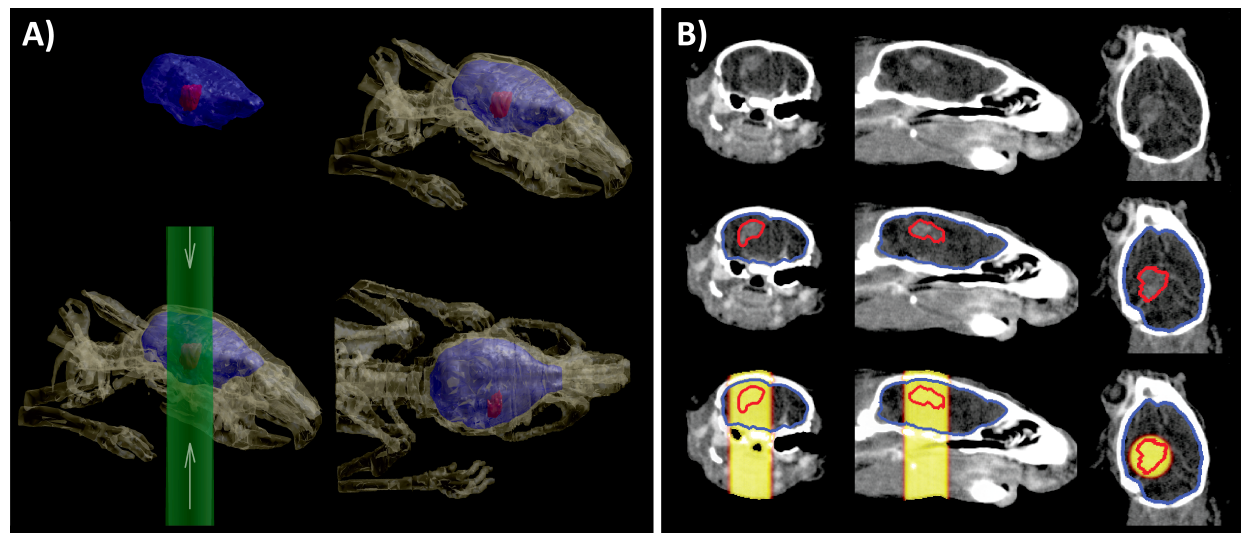

Figure 6.2: A) Visualization of the tumour in the brain, and applied parallel opposed beams to target the tumour. B) Tumour delineation based on contrast-enhanced micro-CT prior to RT. Red contour: tumour, Blue line: normal brain tissue.
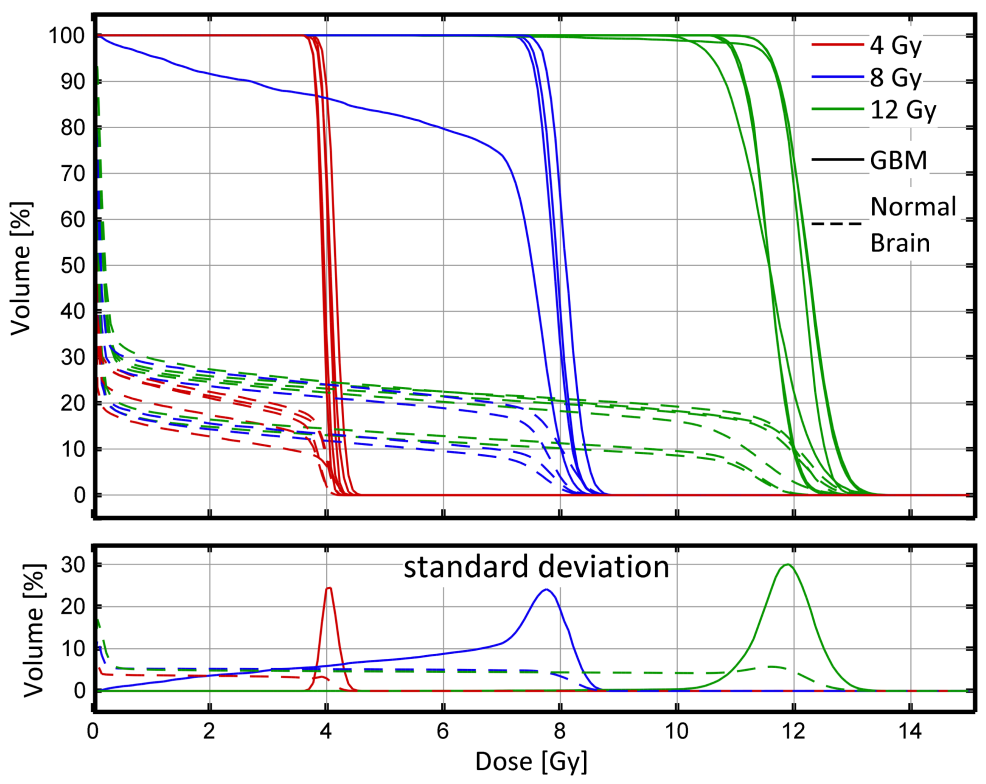

Figure 6.3: Top) Resulting DVHs of the tumours and normal brain tissue (excluding tumour) for all cases. Bottom) Standard deviations of the DVHs per dose group. 
Table 6.1: Dose-volume metrics and structure volumes for the PTV and healthy brain, and applied circular beam size for all cases, sorted per dose group. Average values are provided for each dose group. PTV=planning target volume

\begin{tabular}{|c|c|c|c|c|c|c|c|c|c|}
\hline \multirow[b]{2}{*}{$\begin{array}{c}\text { beam } \varnothing \\
(\mathrm{mm})\end{array}$} & \multicolumn{5}{|c|}{ PTV } & \multicolumn{4}{|c|}{ Normal Brain } \\
\hline & $\begin{array}{c}D_{\text {mean }} \\
(G y)\end{array}$ & $\begin{array}{c}D_{5} \\
(G y)\end{array}$ & $\begin{array}{l}D_{95} \\
(G y)\end{array}$ & $\begin{array}{l}V_{95} \\
(\%)\end{array}$ & $\begin{array}{c}V \\
\left(m m^{3}\right)\end{array}$ & $\begin{array}{c}D_{\text {mean }} \\
(G y)\end{array}$ & $\begin{array}{c}D_{\max } \\
(G y)\end{array}$ & $\begin{array}{l}V_{100} \\
(\%)\end{array}$ & $\begin{array}{c}\mathrm{V} \\
\left(m m^{3}\right)\end{array}$ \\
\hline \multicolumn{10}{|l|}{4 Gy } \\
\hline 5 & 4.1 & 4.3 & 3.9 & 98.2 & 4.0 & 0.9 & 4.6 & 9.0 & 324.1 \\
\hline 5 & 4.0 & 4.2 & 3.9 & 99.5 & 11.0 & 0.9 & 4.4 & 7.7 & 345.9 \\
\hline 3 & 4.2 & 4.4 & 3.9 & 99.8 & 4.1 & 0.5 & 4.7 & 6.3 & 338.4 \\
\hline 5 & 4.0 & 4.1 & 3.8 & 92.5 & 6.7 & 0.7 & 4.4 & 1.7 & 348.5 \\
\hline 5 & - & - & - & - & - & 0.8 & 4.6 & 6.8 & 341.2 \\
\hline 5 & 3.9 & 4.1 & 3.8 & 91.4 & 9.3 & 0.9 & 4.3 & 1.9 & 364.3 \\
\hline Average & 4.0 & 4.2 & 3.9 & 96.3 & 7.0 & 0.8 & 4.5 & 5.6 & 343.7 \\
\hline \multicolumn{10}{|l|}{8 Gy } \\
\hline 3 & 7.9 & 8.3 & 7.6 & 93.4 & 2.6 & 1.1 & 9.0 & 2.6 & 338.4 \\
\hline 3 & 6.6 & 8.1 & 1.2 & 42.6 & 12.7 & 1.0 & 8.8 & 1.5 & 406.1 \\
\hline 5 & 7.9 & 8.3 & 7.5 & 89.3 & 6.5 & 1.7 & 8.9 & 4.0 & 336.0 \\
\hline 5 & - & - & - & - & - & 1.9 & 9.5 & 11.3 & 331.4 \\
\hline 5 & 8.1 & 8.5 & 7.7 & 98.4 & 2.7 & 2.0 & 9.2 & 10.5 & 380.9 \\
\hline Average & 7.6 & 8.3 & 6.0 & 80.9 & 6.1 & 1.6 & 9.1 & 6.0 & 358.5 \\
\hline \multicolumn{10}{|l|}{12 Gy } \\
\hline 5 & 12.2 & 12.9 & 11.6 & 98.6 & 6.7 & 2.8 & 14.1 & 11.1 & 336.9 \\
\hline 3 & 11.6 & 12.1 & 11.0 & 69.3 & 2.6 & 1.4 & 12.8 & 0.3 & 333.0 \\
\hline 5 & 12.2 & 12.9 & 11.6 & 98.5 & 9.7 & 2.7 & 13.8 & 8.9 & 344.2 \\
\hline 5 & 12.1 & 12.6 & 11.6 & 97.0 & 13.0 & 2.9 & 13.5 & 10.2 & 354.4 \\
\hline 3 & 11.6 & 12.6 & 10.6 & 60.4 & 5.6 & 2.4 & 14.5 & 2.9 & 376.8 \\
\hline 5 & - & - & - & - & - & 2.5 & 13.6 & 5.1 & 341.5 \\
\hline 3 & 11.6 & 12.1 & 11.0 & 70.5 & 1.3 & 1.6 & 12.7 & 0.4 & 335.9 \\
\hline Average & 11.9 & 12.5 & 11.2 & 82.4 & 6.5 & 2.3 & 13.6 & 5.6 & 346.1 \\
\hline
\end{tabular}


A)

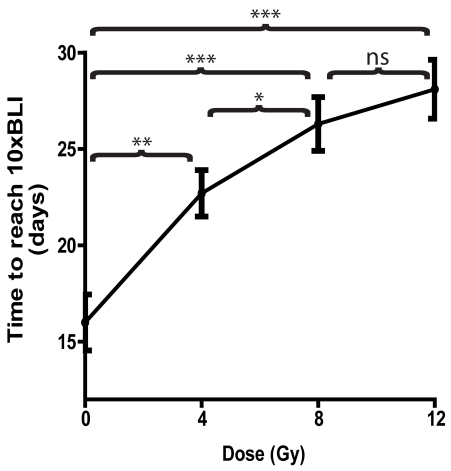

B)

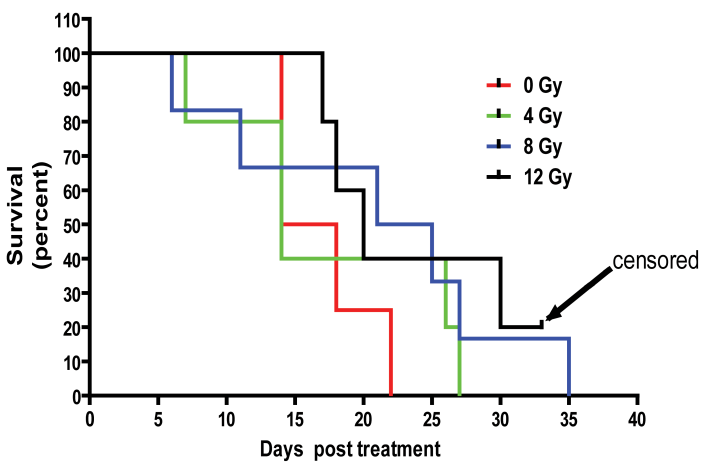

Figure 6.4: Radiation dose-response tumour growth delay and survival. A) Tumour growth delay is normalized to the BLI signal intensity at the start of the treatment. B) Kaplan-Meier curves showing times to reach endpoint measured by neurological and atypical behavior.

tumours were irradiated with either of 4,8 or $12 \mathrm{~Gy}$, based on treatment plans generated using SmART-plan. An example of tumour delineation in axial, coronal and sagittal direction is shown with radiation treatment setup using 2 parallel-opposed beams (figure 6.2A). Tumours were irradiated with $5 \mathrm{~mm}$ or $3 \mathrm{~mm}$ beams, depending on their volume. Resulting dose-volume histograms (DVH) and DVH metrics of the PTV and OAR for all in vivo irradiations are shown in table 6.1. The DVHs indicate clearly that the prescribed dose to the PTV is well achieved, and reveal that about $80 \%$ of the normal brain remains untreated (figure 6.3). The mean dose within the PTV ( $\left.D_{\text {mean }}\right)$ was close to the planned dose with an average prescribed dose of 4.0, 7.6 and 11.9 Gy for 4, 8 and 12 Gy radiation groups, respectively (table 6.1). In one case, a high deviation from the planned dose of $8 \mathrm{~Gy}$ was observed. This was because the tumour volume was not completely covered by the $3 \mathrm{~mm}$ beam (figure 6.5).

To assess the target coverage and normal tissue sparing, we report the $V_{95}$ for the PTV and $\mathrm{V}_{100}$ for the OAR, i.e. the percentages of the volume receiving $95\left(\mathrm{~V}_{95}\right)$ or $100\left(\mathrm{~V}_{100}\right) \%$ of the prescribed dose. To provide additional insight in the uniformity of the delivered dose to the PTV, we report the minimum dose to the hottest $5 \%$ (D5) and $95 \%\left(D_{95}\right)$ of the PTV. The uniformity is consistent among mice in each radiation group, with only one case showing a large variation due to the aforementioned reason. We observed that on average, $96.3 \%, 80.9 \%$, and $82.4 \%$ of the tumour volume prescribed with the planned doses of 4, 8 and 12 Gy, respectively, received $95 \%$ of the prescribed doses $\left(V_{95}\right)$. We noticed that mice irradiated with $3 \mathrm{~mm}$ beams showed lower $\mathrm{V}_{95}$ compared to $5 \mathrm{~mm}$ ones (Table 1). Three mice are lacking information on 
their PTV due to erroneous injection of the contrast agent for the CT image; however the BLI demarcated signal region was used for irradiation using the $5 \mathrm{~mm}$ beam, which could provide a sufficient margin to achieve good tumour coverage. To standardize tumour burden the endpoint was defined as the time to reach 10 times increase in absolute number of photons measured by BLI. Radiation treatment showed a significant dose-response on tumour growth delay measured from an average of 16 days for non-irradiated group to $22.7,26.3$ and 28.1 days for mice receiving a single dose of 4,8 and 12 Gy, respectively (figure 6.4A). By increasing the dose, differences were observed in the survival based on humane endpoints as expressed in the survival curves (figure 6.4B).

\subsection{Discussion}

The micro-invasive nature of GBM into the normal brain makes complete surgical resection impossible, and standard of care for GBM includes radiotherapy and chemotherapy [1]. Advances in molecular imaging using CT-based planning PET [10] and MRI [11] have resulted in improved accuracy of treatment planning and a modest increase in tumour control with reduced normal tissue toxicities. However, more radical improvements on survival are needed and will most likely come from novel therapeutic approaches. To facilitate the rapid translation of radiobiology into clinical practice, novel approaches need to be validated in relevant in vitro as well as preclinical in vivo models using routine clinical procedures. Here we demonstrate an integrated platform for GBM treatment evaluation from in vitro drug screening of treatment combinations to in vivo orthotopic tumour model using small animal image guided radiotherapy planning and precision irradiation. First our study confirms that the 3D spheroid model is a reproducible and robust screening methodology to identify combination therapies for GBM that reflect in vivo response. Dose-responses can be obtained in the spheroid growth delay assays using clinically relevant treatments and characteristics such as TMZ sensitivity and resistance. Gene expression profiles derived from 3D models are reported to be closer to clinical profiles when compared to 2D models [12]. Strong similarities have been shown in drug resistance phenotypes between tumour samples and those observed with 3D spheroid models [13].

These findings provide the rationale for using 3D system to better predict clinical efficacy. In that context, our data showing significant increased efficacy when combining TMZ/RT with Cisplatin are encouraging. Although not commonly used, the 
non-overlapping toxicity profiles of both drugs and the underlying mechanism of action whereby cisplatin reduces DNA-alkyl transferase enzyme activity (responsible for conferring resistance to $\mathrm{TMZ}$ ) offer potential. The combination of Cisplatin with bid $\mathrm{TMZ}$ regimen in chemotherapy-naïve patients with recurrent glioblastoma has shown already to be effective in a Phase II clinical study [14]. Our data in spheroids indicate that adding Cisplatin has also potential in regimens where radiation is part of standard of care.

The 3D models used here however still lack important factors from the tumour microenvironment such as vasculature, fibroblasts or immune cells. Therefore, we used a preclinical orthotopic GBM model and demonstrated the feasibility of image guided radiotherapy using clinical radiation schemes employing image guided small animal micro-irradiators. While the U87MG model lacks important features of human GBM such as micro-invasion, its reproducible tumour growth with short latencies make it an ideal preclinical model to study the in vivo efficacy of novel radiosensitizers in standard of care combination therapies. Models which have more typical GBM characteristics such as primary patient derived models represent important advances which need to be implemented. The effect of radiation on normal brain tissue is a determining factor in GBM treatment because of functional impairment. Considering this and in contrast to standard devices using a single large beam, the use of image guided multiple cross-firing beams on a rotating gantry that deliver a prescribed dose to an isocentre placed at the centre of the target (tumour), results in greater dose homogeneity and less dose at the OAR. Based on the DVHs, the parallel-opposed fields indicate that the beams were highly conformal to a localized brain region. They also demonstrate a highly uniform dose distribution across the tumour, which is particularly useful when trying to establish a dose-response curve in order to identify an appropriate treatment dose that allows room for therapeutic synergies for multimodal treatments. However, the fraction of the beam containing penumbra is greater for the smaller $3 \mathrm{~mm}$ beam than for the $5 \mathrm{~mm}$ beam, which reduced the $\mathrm{V}_{95}$. Therefore, treatment margins should be applied to visible tumours (GTV) for sub-clinical disease spread and uncertainties in treatment delivery. Our study employed a radiation field size that is proportionally more realistic to clinical experience than previously published used whole brain irradiation, which is frequently used in mice.

In conclusion, the use of new high-precision image guided animal irradiation platforms may accelerate the discovery and facilitate the implementation of novel treatment interventions from in vitro screening approaches into clinical testing. 


\section{Conflict of interest}

None.

\section{Acknowledgements}

M.V. and L.B. are supported by ERC Grant 617060. U87MG-R was kindly provided by Dr. W. Leenders (Radboud University, Nijmegen). 


\section{References}

1. Stupp R, Mason WP, van den Bent MJ, Weller M, Fisher B, Taphoorn MJB, Belanger K, Brandes AA, Marosi C, Bogdahn U, Curschmann J, Janzer RC, Ludwin SK, Gorlia T, Allgeier A, Lacombe D, Cairncross JG, Eisenhauer E, Mirimanoff RO. Radiotherapy plus Concomitant and Adjuvant Temozolomide for Glioblastoma. N. Engl. J. Med. 2005; 352: 987-996. doi: 10.1056/NEJMoa043330.

2. Oliva CR, Nozell SE, Diers A, McClugage SG, Sarkaria JN, Markert JM, Darley-Usmar VM, Bailey SM, Gillespie GY, Landar A, Griguer CE. Acquisition of Temozolomide Chemoresistance in Gliomas Leads to Remodeling of Mitochondrial Electron Transport Chain. J. Biol. Chem. 2010; 285: 39759-39767. doi: 10.1074/jbc.M110.147504.

3. Howes AL, Chiang GG, Lang ES, Ho CB, Powis G, Vuori K, Abraham RT. The phosphatidylinositol 3-kinase inhibitor, PX-866, is a potent inhibitor of cancer cell motility and growth in three-dimensional cultures. Mol. Cancer Ther. 2007; 6: 2505-2514. doi: 10.1158/15357163.MCT-06-0698.

4. Carlsson J, Acker $\mathrm{H}$. Relations between ph, oxygen partial pressure and growth in cultured cell spheroids. Int. J. Cancer 1988; 42: 715-720. doi: 10.1002/ijc.2910420515.

5. Eke I, Cordes N. Radiobiology goes 3D: How ECM and cell morphology impact on cell survival after irradiation. Radiother. Oncol. 2011; 99: 271-278. doi: 10.1016/j.radonc.2011.06.007.

6. Verhaegen F, van Hoof S, Granton PV, Trani D. A review of treatment planning for precision image-guided photon beam pre-clinical animal radiation studies. Z. Med. Phys. 2014; 24: 323-334. doi: 10.1016/j.zemedi.2014.02.004.

7. Yahyanejad S, Granton PV, Lieuwes NG, Gilmour L, Dubois L, Theys J, Chalmers AJ, Verhaegen $\mathrm{F}$, Vooijs $\mathrm{M}$. Complementary use of bioluminescence imaging and contrast-enhanced micro-computed tomography in an orthotopic brain tumor model. Mol. Imaging 2014; 13: doi: 10.2310/7290.2014.00038.

8. Hiddingh L, Tannous BA, Teng J, Tops B, Jeuken J, Hulleman E, Boots-Sprenger SH, Vandertop WP, Noske DP, Kaspers GJL, Wesseling P, Wurdinger T. EFEMP1 induces $\gamma$-secretase/Notch-mediated temozolomide resistance in glioblastoma. Oncotarget 2013; 5: 363-374. doi: 10.18632/oncotarget.1620.

9. Van Hoof SJ, Granton PV, Verhaegen F. Development and validation of a treatment planning system for small animal radiotherapy: SmART-Plan. Radiother. Oncol. J. Eur. Soc. Ther. Radiol. Oncol. 2013; 109: 361-366. doi: 10.1016/j.radonc.2013.10.003.

10. Niyazi M, Geisler J, Siefert A, Schwarz SB, Ganswindt U, Garny S, Schnell O, Suchorska B, Kreth F-W, Tonn J-C, Bartenstein P, Fougère C la, Belka C. FET-PET for malignant glioma treatment planning. Radiother. Oncol. 2011; 99: 44-48. doi: 10.1016/j.radonc.2011.03.001.

11. Chang J, Thakur SB, Huang W, Narayana A. Magnetic resonance spectroscopy imaging (MRSI) and brain functional magnetic resonance imaging (fMRI) for radiotherapy treatment planning of glioma. Technol. Cancer Res. Treat. 2008; 7: 349-362.

12. Zietarska M, Maugard CM, Filali-Mouhim A, Alam-Fahmy M, Tonin PN, Provencher DM, MesMasson A-M. Molecular description of a 3D in vitro model for the study of epithelial ovarian cancer (EOC). Mol. Carcinog. 2007; 46: 872-885. doi: 10.1002/mc.20315.

13. Barbone D, Yang T-M, Morgan JR, Gaudino G, Broaddus VC. Mammalian Target of Rapamycin Contributes to the Acquired Apoptotic Resistance of Human Mesothelioma Multicellular Spheroids. J. Biol. Chem. 2008; 283: 13021-13030. doi: 10.1074/jbc.M709698200.

14. Brandes AA, Basso U, Reni M, Vastola F, Tosoni A, Cavallo G, Scopece L, Ferreri AJ, Panucci 
MG, Monfardini S, Ermani M. First-Line Chemotherapy With Cisplatin Plus Fractionated Temozolomide in Recurrent Glioblastoma Multiforme: A Phase II Study of the Gruppo Italiano Cooperativo di Neuro-Oncologia. J. Clin. Oncol. 2004; 22: 1598-1604. doi: 10.1200/JCO.2004.11.019. 


\section{Supplementary material}

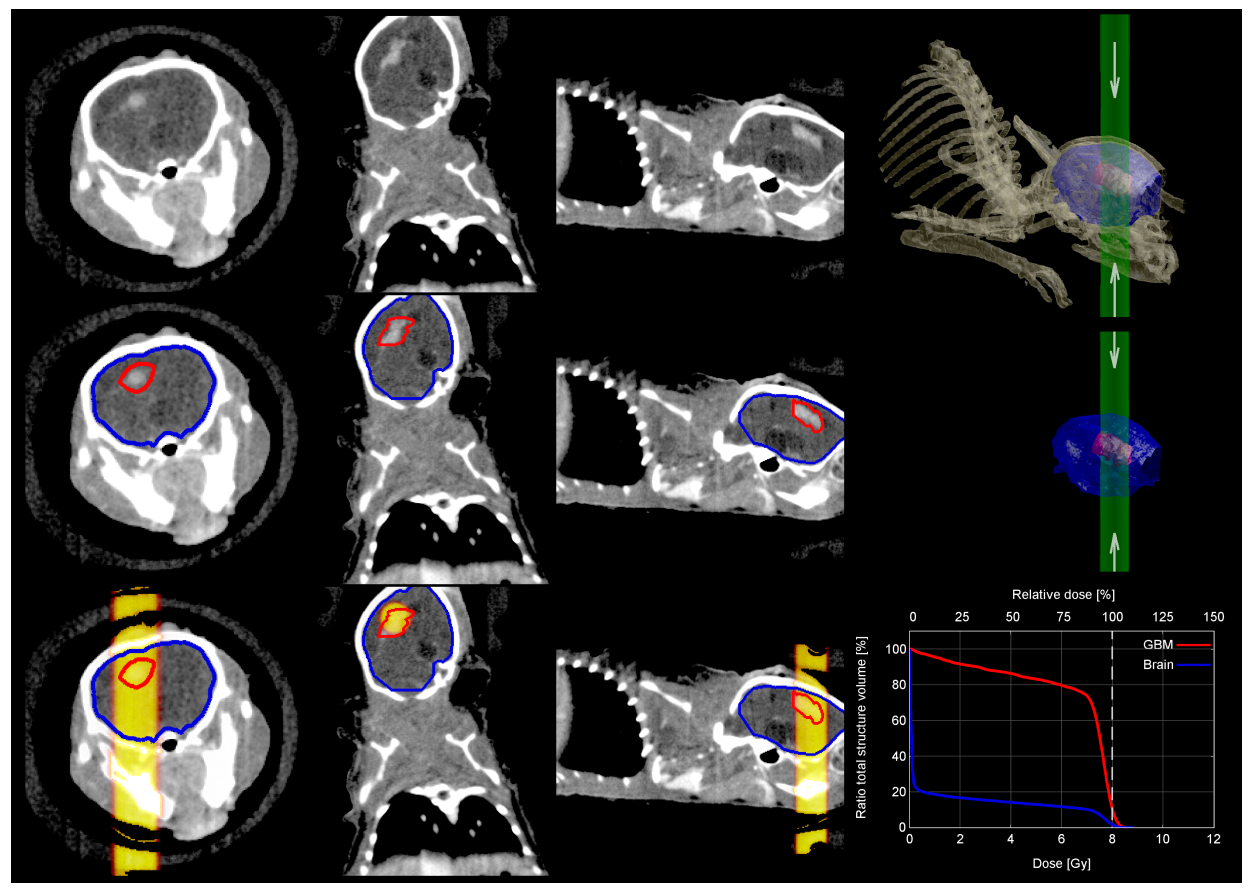

Figure 6.5: Tumour with high deviation from indicated dose. The tumour delineation and calculated dose distribution with the CT image shows that the tumour is not completely covered by the $3 \mathrm{~mm}$ beam. The DVH shows a big deviation from the planned dose (8Gy) to the tumour. 


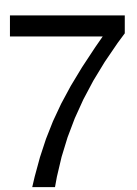

General discussion 



\subsection{New frontiers}

With the introduction of micro-IGRT platforms, a new generation of integrated hardware became available to advance preclinical radiobiological research. The integration of $\mu \mathrm{CT}$ image guidance, beam collimation, anaesthesia systems, and various spatial degrees of freedom for animal and radiation beam positioning enabled conformal dose delivery in the preclinical setting. Additional imaging modality integrations are also rapidly becoming available. With the increasing interest in, and development of, new orthotopic cancer models, powerful software packages for e.g. treatment planning and dose calculation are required to benefit from the full potential of micro-IGRT devices.

It is encouraging that the visualisation and quantification of target and avoidance tissues, beam configurations and calculated dose distributions, incentivizes a pursuit for improved dose delivery conformality using the smallest treatment margins possible. It then becomes important to consider accuracy limits and required treatment margins; what you see is an estimate of the dose distribution that you get. This is in remarkable contrast to traditional animal irradiations during which radiation targeting, conformality and delivered dose uncertainties can be an order of magnitude larger.

\subsection{Practical deployment}

Optimal and efficient use of a micro-IGRT platform requires the input from a varied mix of people with different backgrounds. Clinical practice should be involved to guide research hypotheses, physicists and engineers need to assure, not solely quantify, image guidance and radiation targeting uncertainties, and animal lab technicians and dedicated researchers need to be trained to understand and use the hardware and software properly. Presence and availability of support staff are indispensable for these platforms. I have seen first-hand that our X-RAD 225Cx was often seemingly surrounded by an invisible wall for other researchers, indicating the need to improve the workflow and available tools. Well designed and implemented software is indispensable, software that ideally shouldn't require users to consult the manual.

Such a combination of differently skilled people, for whom it is also possible to dedicate time to the projects, may be the biggest hurdle for many institutes that are setting up precision image guided radiobiological studies. Particularly, these devices need to bring together researchers from the wet and dry lab, who are often physically separated in different departments, buildings or institutes. This takes effort and patience 
to accomplish, to convene and keep such teams together, and to align their biological and technical jargons. It is difficult to understand what is possible for different team members, or perhaps more importantly to know what is impossible. When our micro-IGRT arrived, we were asked to perform three-dimensional heterogeneous irradiations on the millimetre scale, while we were just getting started with depth dose rate lookup tables. Biologists can laugh at physicists' pursuit to skim another percent error margin, and the physicists need to face faeces in their equipment.

Clinical devices undergo an extensive commissioning procedure and detailed annual, monthly and daily quality assurance protocols are performed. Micro-IGRT installations do not benefit from such available human resources and financial streams. Nonetheless, these platforms require regular quality assurance such as dosimetric characterisation and verification, maintenance to high voltage and moving parts, and software updates.

In research grants, it is often not possible to budget for maintenance and support staff. Upfront installation costs, including necessary facility modifications such as floor reinforcements or increased cooling capacity and anaesthesia gas venting, create a big threshold. Especially when the base functionality of these devices needs to be extended with e.g. additional mechanical degrees of freedom for irradiation or bioluminescence imaging. It is also difficult to assure continued use of this expensive research hardware, which makes budgeting for recurring costs difficult as well. Besides these obstacles, it is difficult to consult available knowledge. Literature is scattered throughout journals of which some only relate to this research field. There is also no single database or overview of published work or operating standards available. To help address this, our research group started to organize three-day symposia on Precision Image Guided Small Animal RadioTherapy of which the most recent edition was held in March 2018 in Lisbon, after successful editions in Maastricht (2013), Vancouver (2014) and Ghent (2016). On average, we welcomed more than one hundred attendants from industry and research institutes, mainly from North-America and Europe but more recently also from Asia and Australia, indicating the expansion of the research field. In 2011, we estimated that there were 11 operational micro-IGRT devices worldwide, about 40 in 2014, and currently that number lies well above 100 . We expect that this growth will continue until there are about 250 devices worldwide at research institutes and pharmaceutical companies in perhaps 10 years from now. The first installed micro-IGRT systems are getting replaced by more modern iterations, and some institutes are purchasing a second system due to heavy use. 


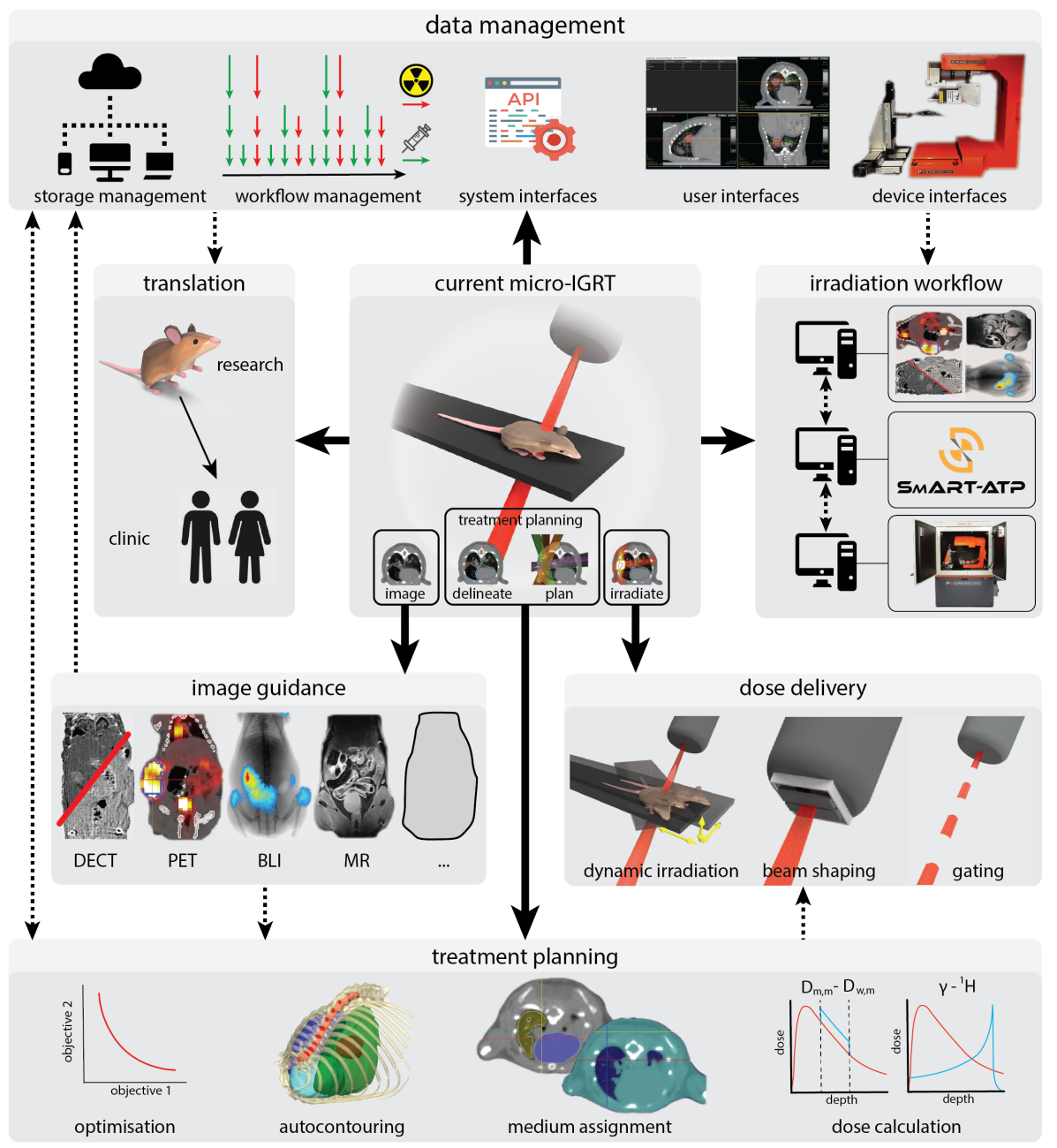

Figure 7.1: We can expect that the field of preclinical radiation therapy will innovate in the illustrated key areas. Innovative and extended hardware for micro-IGRT platforms can improve dose delivery and image guidance. Additional user friendly software is needed to pursue easier, faster and a more robust workflow and to improve overall data management. Preclinical treatment planning will benefit from improved data accessibility and multi-parametric image guidance inputs, which can then be used to optimise treatment plans and consequent irradiation plans. All these elements together are needed to optimise the benefit of research data for clinical translation, to design subsequent clinical trials. 


\subsection{Irradiation hardware}

The current generation micro-IGRT platforms have seen about a decade of evolution and will continue to evolve in the foreseeable future. Important innovative aspects are e.g. image guidance, data management, and irradiation planning. Since the introduction of the first commercial micro-IGRT platform, there have been numerous improvements and changes to the cabinets, such as integrated optical imaging, a decreasing device footprint, many software updates, and extended degrees of freedom for irradiation. An overview of key improving areas is illustrated in figure 7.1.

The commercially available competing devices are the X-RAD SmART, formerly XRAD 225Cx, from Precision Inc. (North Branford, USA) (PXi) and the SARRP from Xstrahl Itd. (Camberley, UK). These systems are similar and converging to the same capabilities. Both systems enable dose delivery from any direction while targeting any three-dimensional isocentre. Both systems use the same x-ray tube (Comet MXR225), use a range of fixed aperture beam collimators, and can be equipped with a jawtype variable aperture collimator and bioluminescence imaging capability. There are however distinct design differences. Cone beam CT image acquisition on the SARRP is performed by coronal rotation of the animal, whereas the X-RAD SmART rotates the gantry in the axial plane of a stationary animal. The X-RAD SmART uses an onboard optical camera mounted on the gantry for bioluminescence imaging, whereas the SARRP requires a separate detachable optical camera setup module, which uses a rotatable 3-mirror system to achieve multi-view image acquisition. In comparison with clinical linacs, micro-IGRT platforms have the possibility to change the irradiation spectrum during irradiation, but lack beam shaping functionality using multi-leaf collimators, and also lack roll and pitch rotation of the subject. There are however several approaches from different research groups and manufacturers to extend the beam shaping functionality. The current standard of practice for beam delivery is the use of circular or rectangular static aperture collimators that need to be inserted manually. $A$ variable aperture collimator would not only increase the flexibility to tune beam sizes to a target volume to improve dose conformality but would also shorten and simplify the workflow. The user would not have to insert and remove the collimator between imaging and irradiation sessions or change the collimator during a multi-beam irradiation protocol with different beam sizes, which reduces error proneness. As mentioned in chapter 1, groups from Stanford University and University of Western Ontario extended a $\mu \mathrm{CT}$ scanner with an iris-like collimation and asymmetrical jaw collimation system, respectively [1, 2]. The group from Johns Hopkins University developed a motorized variable aperture collimator (MVC) in collaboration with XStrahl Ltd. [3, 
4] for the SARRP, and also PXi developed a variable aperture collimator for their XRAD SmART. The extensions for commercial systems are designed to deliver varying rectangularly shaped fields using two sets of orthogonally placed jaws for beam collimation, as illustrated in figure 7.1. A full downscaled version of a clinical multi-leaf collimator (MLC) is not expected to be developed for these devices ever, although an initial design has been published by the research group from University of WisconsinMadison [5, 6]. Manufacturing, radiation leakage, and irradiation quality assurance would become insurmountable for reliable practical application. Instead, a micro-jaw collimation approach for rectangular fields could be used to deliver a superposition of multiple beams of varying sizes to achieve micro-IMRT, such as the group from the department of radiation oncology at the University of California Los Angeles (UCLA) who recently reported on their sparse orthogonal collimator with rectangular aperture optimisation for their X-RAD SmART [7, 8]. It is important to note however, that all micro-variable aperture collimators remain unvalidated in published literature for therapeutic dose delivery.

A downside of such hardware that enables the use of many smaller beams to deliver complex dose distribution is that the required irradiation time to delivery therapeutic doses increases considerably. A dose rate increase for $x$-ray tubes installed on micro-IGRT units is likely to occur and would be the most straightforward approach to decrease required beam-on times. The current $x$-ray tubes use a static electron target (anode). With a rotating target and improved cooling systems, such as those used in modern clinical CT scanners, heat dissipation within the $x$-ray tube can be improved to enable higher currents, and sufficiently powerful high voltage generators are already available. A dose rate increase is beneficial for several purposes. A smaller electron focus closer to an ideal point source increases the achievable imaging resolution. Also, following the same principle, the use of a smaller focal spot for irradiation decreases the beam penumbra sizes. Another pragmatic approach to increase the dose rate is to position the animal closer to the $x$-ray source. The remaining clearance between the end of the collimator and the animal is usually only about $5 \mathrm{~cm}$. However, with automatically calculated irradiation protocols, and with treatment planning software that would be aware of the spatial occurrences of the animal couch, animal, and beam shaping hardware during the treatment, such a source to target distance decrease could be incorporated without any additional effort from the user.

The current commercial micro-IGRT units are equipped with a small and large focal spot for x-ray generation that have a diameter of about 1.0 or $5.5 \mathrm{~mm}$, respectively. The large focal spot offers a higher photon flux at the expense of imaging quality and 
beam penumbra sharpness, but both focal spot distributions are heterogeneous [9]. Stewart et al. reported a penumbra sharpening for the $80 \%-20 \%$ dose falloff region of 0.43 to $0.21 \mathrm{~mm}$ for a $1 \mathrm{~mm}$ diameter beam [10,11], when comparing the resulting beam penumbrae when using the small and large focal spot for irradiation. A smaller focal spot would also reduce the non-uniform fluence in very small fields [9]. Especially when applying a dose painting technique as described in chapter 5 , the dose rate quickly becomes the limiting factor for practical applicable complex treatments. The use of smaller beams, and the use of the small focal spot for irradiation, at higher dose rates can improve achievable dose conformality for the same irradiation time. An extension of the work of the optimisation framework and dose painting techniques presented in chapter 4 and 5 could involve the optimisation with inclusion of the small and large focal spot for irradiation. Higher dose rates of the large focal spot and better spatial dosimetric modulation of the small focal spot can then be included in the same irradiation protocol.

There are a few groups that work on the development of x-ray shutters for micro-IGRT platforms. An x-ray shutter nearly eliminates the ramp up and ramp down times for the dose rate in the target region. A group from Oxford University upgraded the treatment head assembly of their SARRP to include an x-ray shutter [12], and a research group from Cyceron in France developed a beam shutter that can be attached to a fixed aperture collimator of the X-RAD 225Cx [13]. This technique is known as gating and can be applied at the expense of prolonged treatment time, see figure 7.1 for an illustration. Gating can be used to reduce the required CTV-PTV margin due to e.g. breathing motion $[12,13]$. The collimator assembly from Oxford University can work in tandem with an optical fibre for diffuse reflectance measurements to determine the mouse breathing phase in real time to take breathing motion into account, which can be useful for both imaging and irradiation. I do not see a need for such advanced tracking and correction methods in the foreseeable future. The field has just started to mature, and the community could currently benefit more from investigations into the type and magnitude of organ motion related issues, and recommendations and operating procedures on margin management. Initial work from Van der Heijden et al. [14], and Vaniqui et al. [15] on required planning target volume margins for mice lung tumours is valuable and will need to be extended, after which further standard practices can be published. Recently, the ESTRO Advisory Committee in Radiation Oncology Practice (ACROP) issued the first report for preclinical radiotherapy. The resulting publication provides an overview of the current state of the art in image guided preclinical radiotherapy technology, provides recommendations on study reporting, and identifies challenges in the field [16]. 
Clinical proton therapy centres are being built rapidly around the world. Although the idea of using particles for therapeutic purposes is certainly not new, it has only relatively recently seen an introduction in routine clinical practice, and expansion of radiobiological knowledge in the field of particle irradiations is needed. To enable particle irradiation, complex hardware and expensive infrastructure are needed, most often using a cyclotron for particle generation. Such investments are too large for dedication to micro-IGRT devices, but a viable option can be to couple a micro-IGRT to a clinical ion beam. The SARRP has been coupled to a proton beam line by a research group from the University of Washington [17], and a group from the University of Pennsylvania put their SARRP on rails to be dockable to a clinical ion beam [18], to enable image guided preclinical particle irradiations. Our own research group investigated the suitability of using the very recently installed local clinical proton pencil beam scanning facility for use in small animal irradiations [19].

There are many other smaller but important improvements that we can expect to take place in the following years. Mechanical tolerances of the collimator in its holder could be reduced. These tolerances cause imperfect and inconsistent collimator alignment with the focal spot which is problematic for precise and accurate therapeutic dose delivery. The weight imbalance of the gantry and the attached hardware causes flex during gantry rotation which can only be compensated partially. The irradiation workflow can also be made more robust by increasing the number of sensors to detect e.g. correct collimator positioning, x-ray filter selection, irradiation delivery based on monitor units instead of beam-on time, correct animal position, correct anaesthesia administration, and improved feedback during treatment delivery. This would also enable the implementation of collision detection and prevention between the collimator and animal couch, or worse, with the animal itself, an important feature that is currently lacking in all micro-IGRT platforms. Other benefits are further automation such as the mentioned variable collimator, automated $\mathrm{x}$-ray filter selection, and communication between the different software packages for image acquisition and processing, data management, and treatment planning. Such improvements would vastly improve the overall workflow, ease of use, and increase robustness. Since animal lab work often involves repetitive actions, it is especially important to avoid steps that are prone to human error.

\subsection{Image guidance}

The field of precision preclinical localised irradiation is made possible through the integration of proper image guidance which is fundamental for animal setup, radiation 
planning, and animal follow up. CT imaging is the basis and is fast and relatively cheap. Exciting innovations are taking place in this area that can be integrated in micro-IGRT platforms in the future, with techniques such as dual [20, 21], phasecontrast [22], and spectral CT imaging. By combining different photon spectra for imaging, that should be minimally overlapping, multi-parametric volumes can be reconstructed [21]. Resulting data are valuable for improved detection of the elemental composition of the tissues and can improve the accuracy of dose calculations [23].

The integration of optical cameras enable bioluminescence imaging (BLI) and could enable fluorescence imaging in the future. It is already relatively common to use image fusion to register CT images of the animal in its current treatment position to previously acquired image data such as MR, PET or SPECT. The onboard optical camera integration of the X-RAD SmART enables a faster and easier workflow that includes BLI. The optical camera for the SARRP needs to be attached, calibrated, and detached for each optical imaging session which prolongs the workflow considerably to about 40 minutes per animal when using bioluminescence guided irradiation [24]. However, at the moment and in the near future, BLI can only be used for fast and relatively cheap animal follow up, for which it is ideally suited since there is essentially no background noise during measurements. The spatial accuracy of acquired BLI data, particularly of reconstructed three-dimensional BLI distributions, is insufficient for useful and reliable radiation targeting. This is because it is very difficult to reconstruct light propagation through different, unknown, tissues using multi-angular two-dimensional acquisitions as input. The irradiation margin that would need to be incorporated would be relativley large, in the order of several millimetres and thereby defeat the purpose of precision preclinical irradiation.

The onboard electronic portal imaging device (EPID) is currently only used for cone beam CT image guidance but offers additional opportunities for improvements. An interesting use is to improve radiation targeting accuracy. Using the known WinstonLutz animal couch corrections to compensate for mechanical sagging [25] together with gantry-flex corrections that are normally only used to improve cone beam CT image reconstruction accuracy, the position of the centre of the beam on EPID can be calculated. Using a short pre-beam exposure, a correction for the animal couch position can be calculated to correct for specific perturbation corrections such as improper collimator placement or for a small drift of the focal spot [26]. For the X-RAD $225 \mathrm{Cx}$, this approach was reported to improve targeting accuracy to $0.05 \pm 0.05 \mathrm{~mm}$ [26].

Another valuable use is the development of $\mu$-Dose Guided RadioTherapy procedures 
for verification of the actual delivered dose. Initial work from Granton et al. [27] used the EPID to verify whether the intended dose is delivered at the position of the EPID of an X-RAD 225Cx, and Anvari et al. calculated transit doses using the CCD-camera based EPID of their SARRP [28]. Currently, the delivered dose is not verified against planned dose routinely during or after each treatment, but especially when preclinical irradiations become more complex and use tighter irradiation margins, verification becomes an essential part of the irradiation workflow. Another approach is to back project measured photon fluences to calculate the dose distribution within the subject [29]. Such dose guided radiotherapy methods are becoming more common in clinical practice and could be extended to micro-IGRT platforms. Onboard imaging devices can also be used to improve and speed up quality assurance procedures [30], which is especially important in research laboratories where there is limited support staff in general.

\subsection{Software}

Many different drivers, libraries, databases, and software packages are required for proper functioning of micro-IGRT platforms; software is at the core to integrate all different parts of these platforms. In general, we differentiate between two different packages; one package that controls the micro-IGRT hardware, and another that is used for radiation treatment planning. Currently, preclinical radiation planning software needs to catch up with the latest hardware capabilities. Moreover, radiobiological study designs need to catch up with the current capabilities of micro-IGRT hardware and software. There are e.g. only very few research groups that use all current capabilities of preclinical treatment planning software SmART-ATP. Most of the radiobiological study setups are performed using very simple beam configurations with little attention for volume of interest definition or dose evaluation and optimisation, but that is expected to change in the near future.

Instead of using more complex beam shaping hardware to improve dose delivery conformality, another approach is the use of a fixed aperture beam in a dynamic fashion. This dose painting technique where the animal is translated during irradiation is described in detail in chapter 5. Dynamic use of the available degrees of freedom is seen as important next step to improve preclinical precision irradiation using current generation hardware, illustrated by the transition from irradiator types $A-B$ to $C-D$ in figure 5.1. A major advantage of such approaches is that the hardware does not need to be extended or replaced, but only software updates are required to improve 
the capabilities of a platform. When variable aperture collimators are ready for animal irradiations, these would be ideally suited to further increase dose delivery flexibility.

The identification and digital segmentation of target and avoidance volumes is currently the most time-consuming step in the clinical radiotherapy workflow because it predominantly involves manual labour. Capable software is key to improve and shorten this process. Multi-parametric imaging such as multi-energy CT can improve tissue identification, which can be used to feed information to automatic contouring algorithms [31].

Data storage, processing, accessibility, analysis, and availability are big challenges in contemporary clinical practice, especially in the highly technological field of radiotherapy. All these data management aspects are still only in early stages of development in preclinical research. Clinical practice is ahead with more mature systems to e.g. record and verify different steps in the process, but preclinical practice is much more scattered, also for standards on communicating data between different systems. A better implementation of overall data management solutions in preclinical practice would undoubtedly improve and speed up preclinical research and translation to clinical practice [32].

\subsection{Preclinical irradiation planning}

The main difference between preclinical and clinical radiotherapy planning, results from the lower photon energies used. Although kV photon energies are used in clinical practice, such as for orthovoltage skin irradiation or brachy therapy, there are no clinical radiation planning tools available that are suitable for micro-IGRT platforms. For skin irradiation, there is currently no patient specific image guidance or radiation planning being used. While photon energies for brachytherapy can be lower, such as $50 \mathrm{kVp}$, current clinical practice is to use water-based dose calculations [33], which is also problematic [34].

The application of kernel-based dose calculation methods for kV irradiation is fundamentally flawed and should not be used for applications that require high accuracy. Especially in setups that contain high fluctuations of the elemental composition of media, such as in the vicinity of interfaces with air or bone. If Monte Carlo models and radiation transport are validated in e.g. water, then this approach is also valid for other media. This is due to the inherent correct handling of different photon interaction mechanisms in all media, as long as cross sections for interaction and scattering are known. Of course, it is still required and extremely challenging that different tissues 
are identified and assigned correctly. For superposition-convolution dose calculation methods, this is not the case; if such methods are validated in water, they are not consequently valid for other media as well. To improve the accuracy of kernel-based calculation methods, it is needed to include various calculations for e.g. primary and scatter radiation as a function of energy photon energy, and the need to rotate and tilt kernels. To include e.g. focal spot inhomogeneity, more alterations and extensions are needed. An important culprit for accurate MC simulations, is the lack of available information on the composition and density of animal tissues and their distributions especially for different strains, ages, and gender. Technology for tissue analysis, e.g. mass-spectrometry, for these relatively low atomic numbers exists, but is not readily available in the radiotherapy field, and expensive to use otherwise. With the increasing size of the community, perhaps all involved research institutes could co-finance a study to gather these valuable data.

One of the main tangible results from the work of this thesis is unmistakably our developed dedicated Monte Carlo based small animal radiation treatment planning system SmART-Plan. When we embarked on this project about 6 years ago, we could not have imagined the impact this work would have. From the start we saw the unmet need of capable dedicated software that would enable micro-IGRT units to deliver complex dose distributions. With the specific cumulative knowledge present in our group, we thought to be in an ideal position to proceed down this path. Instead of developing tools that would be used solely at our own department, we immediately strove for a product that could be deployed at other institutes as well. We already collaborated with PXi as centre of excellence for their product, and they also supported our efforts to improve preclinical treatment planning. A synergy that is ongoing until today, and that we have since professionalized tremendously.

In June 2013, we proudly finalized the commissioning of the first externally installed version of SmART-Plan at the National Institute of Health in Washington. The project continued to take a large chunk of our research time, and the number of installations grew steadily, and in the beginning of 2017 SmART-Plan was being used at about 15 sites worldwide. By then, we had founded the company SmART Scientific Solutions B.V. to take over this commercial endeavour. New software was built from scratch and named SmART-ATP, for SmART Advanced Treatment Planning. Combined, SmARTPlan and SmART-ATP are now being used at more than 30 institutes across NorthAmerica, Europe, Australia and Asia.

Before SmART-Plan was developed, PXi offered DOSCTP for radiation planning which uses DOSXYZnrc as dose calculation engine as well, but has limited functionality [35]. 
Their competitor XStrahl offers Muriplan, which was built by extending SlicerRT [36], an extension of 3D Slicer [37], and uses a superposition-convolution kernel-based dose calculation engine [38, 39]. Recently, the company RaySearch Laboratories entered the field of small animal radiotherapy planning. RaySearch is a fast-growing radiation treatment planning software company based in Sweden, which licenses their products for clinical radiation planning. At the Fourth International Conference on Precision Image Guided Small Animal Radiotherapy Research in Lisbon in March 2018, they demonstrated $\mu$-RayStation for the specific application to small animal radiotherapy [40]. $\mu$-RayStation is adapted from their clinical radiotherapy planning system RayStation 5 in collaboration with research groups from Dresden and Nantes. $\mu$-RayStation uses the Monte Carlo code XVMC/VMC++ [41, 42], for dose simulations with an analytically modelled irradiator.

\subsection{Conclusion}

The integration of complementary technologies and capable software have enabled localized preclinical image guided radiotherapy. Combined with newly developed biological animal models, these micro-IGRT platforms facilitate the advancement of preclinical radiobiological research using irradiation setups alike contemporary clinical practice. Adapted study designs may then increase the quality, speed, efficiency, and cost effectiveness of the development of novel treatment strategies.

Capable and reliable radiation planning is invaluable for complex and robust in vivo radiobiological studies, to which the developed and validated techniques in this thesis contribute. This was achieved by introducing state of the art MC simulations and radiation plan optimisation for preclinical precision irradiation. These techniques were applied to achieve dynamic irradiation to improve conformality and flexibility of microIGRT platforms. This leads to the conclusion that the work of this thesis enables unprecedented preclinical radiation planning accuracy and quantitative insights, and contributes to enable previously unattainable radiobiological experiments. Developed knowledge and methods were demonstrated by performing localized brain irradiations for an orthotopic glioblastoma model in mice. In addition, it can be concluded that developed knowledge is rapidly distributed to research institutes worldwide through successful valorisation. 


\section{References}

1. Graves EE, Zhou H, Chatterjee R, Keall PJ, Gambhir SS, Contag CH, Boyer AL. Design and evaluation of a variable aperture collimator for conformal radiotherapy of small animals using a microCT scanner. Med. Phys. 2007; 34: 4359-4367. doi: 10.1118/1.2789498.

2. Jensen MD, Hrinivich WT, Jung JA, Holdsworth DW, Drangova M, Chen J, Wong E. Implementation and commissioning of an integrated micro-CT/RT system with computerized independent jaw collimation. Med. Phys. 2013; 40: 081706. doi: 10.1118/1.4812422.

3. Cho N, Wong J, Kazanzides P. Dose Painting with a Variable Collimator for the Small Animal Radiation Research Platform (SARRP). MIDAS J. 2014; 942.

4. Cho BJ. Forward and Inverse Treatment Planning Solutions for Small Animal Radiation Research. 2017; Available from: http://dspace-prod.mse.jhu.edu:8080/handle/1774.2/58687.

5. Prajapati S, Mackie T, Jeraj R. Open-Source Medical Devices (OSMD) Design of a Small Animal Radiotherapy System. J. Phys. Conf. Ser. 2014; 489: doi: 10.1088/1742-6596/489/1/012017.

6. Prajapati S, Cox B, Swader R, Petry G, Eliceiri KW, Jeraj R, Mackie TR. Design of an OpenSource Binary Micromultileaf Collimator for a Small Animal Microradiotherapy System. J. Med. Devices 2017; 11: 041007-041007-10. doi: 10.1115/1.4038017.

7. Woods K, Nguyen D, Neph R, O'Connor D, Sheng K. A Sparse Orthogonal Collimator for Small Animal IMRT Using Rectangular Aperture Optimization. Int. J. Radiat. Oncol. • Biol. • Phys. 2018; 102: S152-S153. doi: 10.1016/j.jijobp.2018.06.368.

8. Nguyen D, Ruan D, O'Connor D, Woods K, Low DA, Boucher S, Sheng K. A novel software and conceptual design of the hardware platform for intensity modulated radiation therapy. Med. Phys. 2016; 43: 917-929. doi: 10.1118/1.4940353.

9. Granton PV, Verhaegen F. On the use of an analytic source model for dose calculations in precision image-guided small animal radiotherapy. Phys. Med. Biol. 2013; 58: 3377. doi: 10.1088/0031-9155/58/10/3377.

10. Stewart J, Lindsay P, Jaffray D. WE-E-BRE-04: Dual Focal Spot Dose Painting for Precision Preclinical Radiobiological Investigations. Med. Phys. 2014; 41: 504-504. doi: 10.1118/1.4889433.

11. Stewart JMP. Spatially Modulated Dose Optimization and Performance Limitations with Robust Targeting Performance for Preclinical Irradiation. 2017;

12. Hill MA, Thompson JM, Kavanagh A, Tullis IDC, Newman RG, Prentice J, Beech J, Gilchrist S, Smart S, Fokas E, Vojnovic B. The Development of Technology for Effective Respiratory-Gated Irradiation Using an Image-Guided Small Animal Irradiator. Radiat. Res. 2017; 188: 247-263. doi: 10.1667/RR14753.1.

13. Frelin A-M, Beaudouin V, Le Deroff C, Roger T. Implementation and evaluation of respiratory gating in small animal radiotherapy. Phys. Med. Biol. 2018; 63: 215024. doi: 10.1088/13616560/aae760.

14. Van der Heyden B, van Hoof SJ, Schyns LEJR, Verhaegen F. The influence of respiratory motion on dose delivery in a mouse lung tumour irradiation using the 4D MOBY phantom. Br. J. Radiol. 2017; 90: 20160419. doi: 10.1259/bjr.20160419.

15. Vaniqui A, van der Heyden B, Almeida IP, Schyns LE, van Hoof SJ, Verhaegen F. On the determination of planning target margins due to motion for mice lung tumours using a fourdimensional MOBY phantom. Br. J. Radiol. 2018; 20180445. doi: 10.1259/bjr.20180445.

16. Verhaegen F, Dubois L, Gianolini S, Hill MA, Karger CP, Lauber K, Prise KM, Sarrut D, Thorwarth D, Vanhove C, Vojnovic B, Weersink R, Wilkens JJ, Georg D. ESTRO 
ACROP: Technology for precision small animal radiotherapy research: Optimal use and challenges. Radiother. Oncol. J. Eur. Soc. Ther. Radiol. Oncol. 2018; 126: 471-478. doi: 10.1016/j.radonc.2017.11.016.

17. Ford E, Emery R, Huff D, Narayanan M, Schwartz J, Cao N, Meyer J, Rengan R, Zeng J, Sandison G, Laramore G, N Mayr. An image-guided precision proton radiation platform for preclinical in vivo research. Phys. Med. Biol. 2017; 62: 43. doi: 10.1088/1361-6560/62/1/43.

18. Welcome to the SARRP, Small Animal Radiation Research Platform (SARRP), Perelman School of Medicine at the University of Pennsylvania. Available from: https://www.med.upenn.edu/sarrp/.

19. Almeida IP, Vaniqui A, Schyns LE, van der Heyden B, Cooley J, Zwart T, Langenegger A, Verhaegen $\mathrm{F}$. Exploring the feasibility of a clinical proton beam with an adaptive aperture for pre-clinical research. Br. J. Radiol. 2018; 20180446. doi: 10.1259/bjr.20180446.

20. Landry G, Reniers B, Granton PV, van Rooijen B, Beaulieu L, Wildberger JE, Verhaegen F. Extracting atomic numbers and electron densities from a dual source dual energy CT scanner: experiments and a simulation model. Radiother. Oncol. J. Eur. Soc. Ther. Radiol. Oncol. 2011; 100: 375-379. doi: 10.1016/j.radonc.2011.08.029.

21. Schyns LEJR, Almeida IP, van Hoof SJ, Descamps B, Vanhove C, Landry G, Granton PV, Verhaegen $\mathrm{F}$. Optimizing dual energy cone beam CT protocols for preclinical imaging and radiation research. Br. J. Radiol. 2017; 90: 20160480. doi: 10.1259/bjr.20160480.

22. Bravin A, Coan P, Suortti P. X-ray phase-contrast imaging: from pre-clinical applications towards clinics. Phys. Med. Biol. 2012; 58: R1-R35. doi: 10.1088/0031-9155/58/1/R1.

23. Vaniqui A, Schyns LEJR, Almeida IP, van der Heyden B, van Hoof SJ, Verhaegen F. The impact of dual energy CT imaging on dose calculations for pre-clinical studies. Radiat. Oncol. Lond. Engl. 2017; 12: 181. doi: 10.1186/s13014-017-0922-9.

24. Zhang B, Wang KK-H, Yu J, Eslami S, lordachita I, Reyes J, Malek R, Tran PT, Patterson MS, Wong JW. Bioluminescence Tomography-Guided Radiation Therapy for Preclinical Research. Int. J. Radiat. Oncol. Biol. Phys. 2016; 94: 1144-1153. doi: 10.1016/j.jrobp.2015.11.039.

25. Lutz W, Winston KR, Maleki N. A system for stereotactic radiosurgery with a linear accelerator. Int. J. Radiat. Oncol. Biol. Phys. 1988; 14: 373-381.

26. Stewart JMP, Ansell S, Lindsay PE, Jaffray DA. Online virtual isocenter based radiation field targeting for high performance small animal microirradiation. Phys. Med. Biol. 2015; 60: 9031-9046. doi: 10.1088/0031-9155/60/23/9031.

27. Granton PV, Podesta M, Landry G, Nijsten S, Bootsma G, Verhaegen F. A combined dose calculation and verification method for a small animal precision irradiator based on onboard imaging. Med. Phys. 2012; 39: 4155-4166. doi: 10.1118/1.4725710.

28. Anvari A, Poirier $Y$, Sawant A. Kilovoltage transit and exit dosimetry for a small animal imageguided radiotherapy system using built-in EPID. Med. Phys. 2018; 45: 4642-4651. doi: 10.1002/mp.13134.

29. van Elmpt WJC, Nijsten SMJJG, Schiffeleers RFH, Dekker ALAJ, Mijnheer BJ, Lambin P, Minken AWH. A Monte Carlo based three-dimensional dose reconstruction method derived from portal dose images. Med. Phys. 2006; 33: 2426-2434. doi: 10.1118/1.2207315.

30. Anvari A, Poirier Y, Sawant A. Development and implementation of EPID-based quality assurance tests for the small animal radiation research platform (SARRP). Med. Phys. 2018; 45: 3246-3257. doi: 10.1002/mp.12939.

31. van der Heyden B, Podesta M, Eekers DB, Vaniqui A, Almeida IP, Schyns LE, van Hoof SJ, Verhaegen $\mathrm{F}$. Automatic multiatlas based organ at risk segmentation in mice. Br. J. Radiol. 
2018; 20180364. doi: 10.1259/bjr.20180364.

32. Persoon L, Hoof S van, van der Kruijssen F, Granton P, Sanchez Rivero A, Beunk H, Dubois L, Doosje J-W, Verhaegen F. A novel data management platform to improve image-guided precision preclinical biological research. Br. J. Radiol. 2018; 20180455. doi: 10.1259/bjr.20180455.

33. Rivard MJ, Coursey BM, DeWerd LA, Hanson WF, Huq MS, Ibbott GS, Mitch MG, Nath R, Williamson JF. Update of AAPM Task Group No. 43 Report: A revised AAPM protocol for brachytherapy dose calculations. Med. Phys. 2004; 31: 633-674. doi: 10.1118/1.1646040.

34. Beaulieu L, Carlsson Tedgren A, Carrier J-F, Davis SD, Mourtada F, Rivard MJ, Thomson RM, Verhaegen F, Wareing TA, Williamson JF. Report of the Task Group 186 on model-based dose calculation methods in brachytherapy beyond the TG-43 formalism: current status and recommendations for clinical implementation. Med. Phys. 2012; 39: 6208-6236. doi: 10.1118/1.4747264.

35. Chow JCL, Leung MKK. Treatment planning for a small animal using Monte Carlo simulation. Med. Phys. 2007; 34: 4810-4817. doi: 10.1118/1.2805254.

36. Pinter C, Lasso A, Wang A, Jaffray D, Fichtinger G. SlicerRT: radiation therapy research toolkit for 3D Slicer. Med. Phys. 2012; 39: 6332-6338. doi: 10.1118/1.4754659.

37. Fedorov A, Beichel R, Kalpathy-Cramer J, Finet J, Fillion-Robin J-C, Pujol S, Bauer C, Jennings D, Fennessy F, Sonka M, Buatti J, Aylward S, Miller JV, Pieper S, Kikinis R. 3D Slicer as an Image Computing Platform for the Quantitative Imaging Network. Magn. Reson. Imaging 2012; 30: 1323-1341. doi: 10.1016/j.mri.2012.05.001.

38. Jacques R, Taylor R, Wong J, McNutt T. Towards real-time radiation therapy: GPU accelerated superposition/convolution. Comput. Methods Programs Biomed. 2010; 98: 285-292. doi: 10.1016/j.cmpb.2009.07.004.

39. Jacques R, Wong J, Taylor R, McNutt T. Real-time dose computation: GPU-accelerated source modeling and superposition/convolution. Med. Phys. 2011; 38: 294-305. doi: 10.1118/1.3483785.

40. RaySearch releases micro-RayStation for pre-clinical research - RaySearch Laboratories. Available from: https://www.raysearchlabs.com/press/?year=2017\&cisionid=2773432.

41. Kawrakow I, Fippel M, Friedrich K. 3D electron dose calculation using a Voxel based Monte Carlo algorithm (VMC). Med. Phys. 1996; 23: 445-457. doi: 10.1118/1.597673.

42. Gardner J, Siebers J, Kawrakow I. Dose calculation validation of Vmc++ for photon beams. Med. Phys. 2007; 34: 1809-1818. doi: 10.1118/1.2714473.

43. Yahyanejad S, van Hoof SJ, Theys J, Barbeau LMO, Granton PV, Paesmans K, Verhaegen F, Vooijs M. An image guided small animal radiation therapy platform (SmART) to monitor glioblastoma progression and therapy response. Radiother. Oncol. J. Eur. Soc. Ther. Radiol. Oncol. 2015; 116: 467-472. doi: 10.1016/j.radonc.2015.06.020.

44. Sosa Iglesias V, van Hoof SJ, Vaniqui A, Schyns LE, Lieuwes N, Yaromina A, Spiegelberg L, Groot AJ, Verhaegen F, Theys J, Dubois L, Vooijs M. An orthotopic non-small cell lung cancer model for image-guided small animal radiotherapy platforms. Br. J. Radiol. 2018; 20180476. doi: 10.1259/bjr.20180476.

45. Balvert M, van Hoof SJ, Granton PV, Trani D, den Hertog D, Hoffmann AL, Verhaegen F. A framework for inverse planning of beam-on times for 3D small animal radiotherapy using interactive multi-objective optimisation. Phys. Med. Biol. 2015; 60: 5681-5698. doi: 10.1088/00319155/60/14/5681. 

Summary 

The integration of technologies for preclinical image guidance and precision irradiation complemented with capable software has enabled localized small animal irradiations. Novel biological orthotopic animal models enable improved radiobiological insights. Combined, these advances effected the emergence of a new research area wherein novel treatment approaches, schemes, and combinations, and normal tissue toxicity can be studied. This new research contributes to the increasing relevance of preclinical research data, which may help to reduce the number of failing approaches and therefore expedite clinical translation, and reduce overall animal burden. Accurate and versatile radiation planning methods are indispensable to maximise the benefits of such radiobiological studies.

The work of this thesis advances techniques that improve the accuracy and versatility of preclinical radiation planning, to enable planning and delivery of more flexible and complex dose distributions for preclinical radiotherapy. Monte Carlo based radiation planning is introduced to achieve accurate dose calculation at the appropriate scale and photon energies used. A beam-on time optimisation framework is developed to achieve a higher degree of planning automation which is essential when moving to more complex beam delivery protocols. These techniques are extended by using multiangled irradiation with a fixed beam shape and size, and subject translation during irradiation to enable more conformal dynamic target irradiation. The developed methods are applied to enable localized irradiation for an orthotopic glioblastoma mouse model. All developed knowledge is rapidly distributed to research institutes worldwide through successful valorisation. 

Samenvatting 

De integratie van technologieën voor preklinische beeldgeleiding en precisiebestraling, aangevuld met de juiste software, heeft gelokaliseerde bestralingen van kleine dieren mogelijk gemaakt. Nieuwe orthotope biologische diermodellen verbeteren radiobiologische inzichten. De combinatie van deze innovaties heeft geleid tot het opkomen van een nieuw onderzoeksgebied waarin nieuwe behandelingsstrategieën, -schema's, en -combinaties, en normale-weefsel toxiciteiten kunnen worden bestudeerd. Zulke nieuwe studies helpen om de waarde van preklinische onderzoeksdata te verbeteren, wat kan bijdragen om het aantal falende benaderingen voor nieuwe behandelingsstrategieën te verminderen. Dit helpt om klinische translatie te versnellen, en om de algehele last voor proefdieren te verminderen. Nauwkeurige en flexibele methodes voor stralingsplanning zijn onmisbaar om de bruikbaarheid van radiobiologische studies te maximaliseren.

Het werk waarop deze thesis is gebaseerd verbetert technieken om de nauwkeurigheid en flexibiliteit van preklinische stralingsplanning te verbeteren, en om de planning en afgifte van complexe dosisdistributies mogelijk te maken. Er is gebruik gemaakt van stralingsplanning gebaseerd op Monte Carlo methodes om nauwkeurige dosisberekeningen op de benodigde geometrische schaal, en met de correcte fotonenenergieën te bereiken. $\mathrm{Er}$ is een optimalisatieraamwerk voor bestralingstijden ontwikkeld om de automatisering van stralingsplanning te verbeteren, wat essentieel is om complexere bestralingsplannen mogelijk te maken. Deze technieken zijn verder ontwikkeld door gebruik te maken van bestralingen met een vaste bundelvorm en -grootte vanuit meerdere hoeken, waarbij het bestralingsdoelgebied getransleerd wordt tijdens de bestraling. Hiermee worden dynamische bestralingsplannen gerealiseerd die beter aansluiten bij het doelgebied. De ontwikkelde technieken zijn toegepast om gelokaliseerde bestralingen uit te voeren in een biologisch muismodel voor glioblastoma multiforme. De ontwikkelde kennis is snel ingezet in onderzoekscentra wereldwijd door succesvolle valorisatie. 

Valorisation 



\section{Societal value}

The relevance of advanced and accessible radiation planning tools for image guided small animal radiotherapy research platforms has been outlined in the first chapter of this thesis. This is an invaluable link for the overall innovation in cancer therapy, albeit with indirect and deferred ultimate benefit for patients. The magnitude of current and future numbers of patients worldwide assures the societal impact of the work of this thesis in the long term. In addition, there is an important general trend to minimize the number of animals used for research to decrease overall animal burden and cost of studies, for which it is essential to maximise the quality and value of animal research data. New approaches and tools as presented in this thesis are then indispensable.

\section{Innovation and knowledge utilisation}

Simply defining a micro irradiator in a clinical TPS is insufficient to reach adequate planning accuracy for reasons discussed in chapter 1 and 7 . During the early stage of the work for this thesis, there was no commercial software available for image guided treatment planning in this setting. Research groups were forced to use simpler tabular lookup calculations or develop their own tools. Both options entail that the advanced imaging and irradiation hardware would not be used to their full extent, or that a big investment of time and money are needed to create capable tools. The manufacturers of the X-RAD 225Cx and SARRP were still in the early stages of development of the hardware and had no resources, time or specific knowledge readily available for such developments.

Therefore, we implemented algorithms and knowledge in a user-friendly manner, to advance ongoing radiobiological studies. To achieve results fast with minimal resources, SmART-Plan was written in a high-level interpreted coding language. Its development and validation are the topic of chapter 3 . The vast knowledge on Monte Carlo modelling and simulations for kilovolt $x$-rays was made accessible to users who did not have specific in-depth knowledge of radiation planning and verification. Big efforts were made to make the product available to other research groups who had purchased the same irradiation platform. For marketing and first line customer relations, a collaboration was established with PXi. SmART-Plan would be sold as an add-on for new customers of their X-RAD 225Cx, which later evolved into the X-RAD SmART. With this setup, we had created a unique setting in which we could rapidly apply any developed knowledge to about half of the research community in this specific field throughout the world, using remote software updates. Towards the end of 
the work of this thesis, SmART-Plan was operational at about 20 institutes throughout Europe, North-America, Asia and Australia, and we had presented our work during several international scientific conferences in the Netherlands, Belgium, Spain, Milan, Switzerland, and Portugal. SmART-Plan has already been used for many published studies [1-21], and many more studies are currently in progress or planned. Such studies are difficult to track as discussed in chapter 7 .

\section{Commercialisation}

Successful valorisation of the developed software means that many hours need to be dedicated to the project. Valorisation is encouraged, but a research group or institute is often not equipped for such activities. Therefore, SmART Scientific Solutions BV was created as mentioned in chapter 7, which is part of the Brightlands Maastricht Health Campus [22]. SmART Scientific Solutions BV licenses the rights for ongoing extension, expansion, and development of irradiation planning for image guided small animal radiotherapy and created the new product SmART-ATP from scratch.

Development of software means that there is a huge cost until the first invoice for the created product can be sent to a customer. Once a first version of the product is ready, it is relatively inexpensive to deploy the same version of the product to additional customers. However, for SmART-ATP, a thorough specific calibration and validation procedure must be completed for each customer before accurate radiation planning is possible. Also, costs for customer support grow with the customer database and there will be ongoing product development. Modern consumer or business software is often sold using a licensing model with recurring license fees which makes a lot of sense from the perspective of the commercial entity to assure continuity. However, in the specific market for SmART-ATP, such costs are difficult to budget for, as purchases are usually realized with money from research budgets. It is practically more feasible to include the purchase of additional software together with the purchase of the relatively expensive hardware.

\section{Market}

Despite the number of radiobiological studies being conducted worldwide, the potential market for dedicated small animal radiotherapy planning software is relatively small. In 2013, we estimated that there were about 800 academic medical centres in Europe and the USA combined, of which about three quarter had a radiation research line. Only a part of these research centres would be able to benefit or be interested 
in precision irradiation. That means that the total worldwide market would be limited to a few hundred devices. This makes commercially viable products, and companies based thereon, difficult to realize. Developments must be carried out lean or using external funding. In comparison with clinical counterparts of hardware and software, the potential revenue is very small which is likely one of the main reasons the current established companies in the field do not seem to be keen to enter this market.

Currently, there are about 130 installed commercial devices from Xstrahl and PXi combined. It is expected that this market will be saturated relatively soon, after which revenue for new installs will minimize and new pools of revenue need to be generated from recurring license fees for ongoing updates, upgrades with new feature sets, optional add-ons, additional users, or replacement of current setups. More recently, some larger institutes have shown interest in additional small animal precision irradiation platforms, e.g. because they are physically scattered.

Nevertheless, this means that required investments for research and development have a high impact on the cost of the product. This slows down technical innovations that may otherwise be relatively straightforward to implement. The commercial platforms already cost well over half a million US dollars, and considerably more if a platform is extended with options such as bioluminescence imaging or a more advanced beam collimation system. This is of course an important reason why there have been numerous local non-commercial projects to assemble image guided small animal irradiation platforms as outlined in chapter 1 .

The vast majority of the current preclinical radiation research platforms are being operated at academic institutes, and there are only a handful operational devices at e.g. pharmaceutical companies. Small companies that need animal research are often spin-offs from academic institutes and outsource such studies to their affiliate universities. Large companies often choose to outsource animal studies to bigger contract research organisations (CROs) because they would not only need to invest in the specific required hardware and software but would also need to set up a research laboratory with specific knowledge and skills.

Xstrahl developed the small animal radiotherapy planning system Muriplan for their SARRP, which is based on 3DSlicer [23, 24]. Currently, SmART-ATP can only be used in combination with the X-RAD SmART, and Muriplan can only be used in combination with the SARRP. At the end of 2017, the company RaySearch Laboratories announced their product $\mu$-Raystation as discussed in chapter 7 [25]. $\mu$-Raystation is currently in use at Institut Cancérologie de l'Ouest (Nantes, France) for irradiation 
with their X-RAD 225Cx, and at OncoRay National Center for Radiation Research in Oncology (Dresden, Germany) in combination with their locally developed SAIGRT.

\section{Future}

The market for precision small animal irradiation is not yet saturated and the number of commercial and non-commercial research platforms will keep growing the coming years. The potential market size may grow considerably in the future if e.g. legislation for new therapy or compound approval would specifically demand targeted localized micro irradiation studies with clinically relevant organ at risk avoidance, which is not the case at this time. Another interesting development might be an increasing interest in therapeutic irradiation for companion animals. This is currently still very uncommon in Europe with only about 10-20 irradiation facilities for animals, but higher demands exist in e.g. the USA.

The modern age of commercial software has seen a tremendous move to serverside based software, leading to many products being offered as a service (-aaS). The worldwide internet connectivity improvements with lower latencies and higher bandwidths enabled clients to use CPU and data heavy applications on a server, or in the cloud, without the need for local installation and setup. Instead of purchasing a one-time perpetual software license, a recurring license fee is charged for using the service. Relevant examples in the field of radiotherapy are an online radiochromic film analysis tool [26], cloud-based radiomics analysis software [27], or the ESTRO online tissue contouring training tool FALCON [28]. Providing a product as a cloud-based service has the advantage that is it easier to maintain for the developer. Such a path could also be realized for small animal radiation planning. Prior to the radiation planning, the relevant imaging data would need to be uploaded to a server, but thereafter the calculation may use the more powerful computer resources from the server, and the irradiation plan is easily communicated using small data packages.

When irradiation hardware evolves, or when new software features are being developed, substantial feature sets such as automated contouring using machine learning, can be sold as add-ons, or as paid software upgrades instead of updates, in addition to e.g. training or educational services. Undoubtedly, the radiation planning software will need to be updated regularly to provide state of the art capabilities. 


\section{References}

1. Sosa Iglesias V, van Hoof SJ, Vaniqui A, Schyns LE, Lieuwes N, Yaromina A, Spiegelberg L, Groot AJ, Verhaegen F, Theys J, Dubois L, Vooijs M. An orthotopic non-small cell lung cancer model for image-guided small animal radiotherapy platforms. Br. J. Radiol. 2018; 20180476. doi: 10.1259/bjr.20180476.

2. Frelin A-M, Beaudouin V, Le Deroff C, Roger T. Implementation and evaluation of respiratory gating in small animal radiotherapy. Phys. Med. Biol. 2018; 63: 215024. doi: 10.1088/13616560/aae760.

3. Ashcraft KA, Miles D, Sunday ME, Choudhury KR, Young KH, Palmer GM, Patel P, Woska EC, Zhang R, Oldham M, Dewhirst MW, Koontz BF. Development and Preliminary Evaluation of a Murine Model of Chronic Radiation-Induced Proctitis. Int. J. Radiat. Oncol. 2018; 101: 1194-1201. doi: 10.1016/j.jprobp.2018.04.061.

4. Vaniqui A, van der Heyden B, Almeida IP, Schyns LE, van Hoof SJ, Verhaegen F. On the determination of planning target margins due to motion for mice lung tumours using a fourdimensional MOBY phantom. Br. J. Radiol. 2018; 20180445. doi: 10.1259/bjr.20180445.

5. Ceelen JJM, Schols AMWJ, Kneppers AEM, Rosenbrand RPHA, Drożdż MM, van Hoof SJ, de Theije CC, Kelders MCJM, Verhaegen F, Langen RCJ. Altered protein turnover signaling and myogenesis during impaired recovery of inflammation-induced muscle atrophy in emphysematous mice. Sci. Rep. 2018; 8: 10761. doi: 10.1038/s41598-018-28579-4.

6. Beera KG, Li Y-Q, Dazai J, Stewart J, Egan S, Ahmed M, Wong CS, Jaffray DA, Nieman BJ. Altered brain morphology after focal radiation reveals impact of off-target effects: implications for white matter development and neurogenesis. Neuro-Oncol. 2018; 20: 788-798. doi: 10.1093/neuonc/nox211.

7. Chiu TD, Arai TJ, Campbell lii J, Jiang SB, Mason RP, Stojadinovic S. MR-CBCT image-guided system for radiotherapy of orthotopic rat prostate tumors. PloS One 2018; 13: e0198065. doi: 10.1371/journal.pone.0198065.

8. Belliveau J-G, Jensen MD, Stewart JMP, Solovey I, Klassen LM, Bauman GS, Menon RS. Prediction of radiation necrosis in a rodent model using magnetic resonance imaging apparent transverse relaxation. Phys. Med. Biol. 2018; 63: 035010. doi: 10.1088/1361-6560/aaa034.

9. Vaniqui A, Schyns LEJR, Almeida IP, van der Heyden B, van Hoof SJ, Verhaegen F. The impact of dual energy CT imaging on dose calculations for pre-clinical studies. Radiat. Oncol. Lond. Engl. 2017; 12: 181. doi: 10.1186/s13014-017-0922-9.

10. Yoon SW, Cramer CK, Miles DA, Reinsvold MH, Joo KM, Kirsch DG, Oldham M. A precision 3D conformal treatment technique in rats: Application to whole-brain radiotherapy with hippocampal avoidance. Med. Phys. 2017; 44: 6008-6017. doi: 10.1002/mp.12533.

11. Chaudary N, Pintilie M, Jelveh S, Lindsay P, Hill RP, Milosevic M. Plerixafor Improves Primary Tumor Response and Reduces Metastases in Cervical Cancer Treated with Radio-Chemotherapy. Clin. Cancer Res. 2017; 23: 1242-1249. doi: 10.1158/1078-0432.CCR-16-1730.

12. van der Heyden B, van Hoof SJ, Schyns LEJR, Verhaegen F. The influence of respiratory motion on dose delivery in a mouse lung tumour irradiation using the 4D MOBY phantom. Br. J. Radiol. 2017; 90: 20160419. doi: 10.1259/bjr.20160419.

13. De Ruysscher D, Granton PV, Lieuwes NG, van Hoof S, Wollin L, Weynand B, Dingemans A-M, Verhaegen F, Dubois L. Nintedanib reduces radiation-induced microscopic lung fibrosis but this cannot be monitored by CT imaging: A preclinical study with a high precision image-guided irradiator. Radiother. Oncol. J. Eur. Soc. Ther. Radiol. Oncol. 2017; 124: 482-487. doi: 
10.1016/j.radonc.2017.07.014.

14. Ceelen JJM, Schols AMWJ, van Hoof SJ, de Theije CC, Verhaegen F, Langen RCJ. Differential regulation of muscle protein turnover in response to emphysema and acute pulmonary inflammation. Respir. Res. 2017; 18: 75. doi: 10.1186/s12931-017-0531-z.

15. Chaudary N, Pintilie M, Hedley D, Hill RP, Milosevic M, Mackay H. Hedgehog inhibition enhances efficacy of radiation and cisplatin in orthotopic cervical cancer xenografts. Br. J. Cancer 2017; 116: 50-57. doi: 10.1038/bjc.2016.383.

16. Yoon SW, Miles D, Cramer C, Reinsvold M, Kirsch D, Oldham M. Treatment planning and 3D dose verification of whole brain radiation therapy with hippocampal avoidance in rats. J. Phys. Conf. Ser. 2017; 847: 012004. doi: 10.1088/1742-6596/847/1/012004.

17. Yahyanejad S, King H, Iglesias VS, Granton PV, Barbeau LMO, van Hoof SJ, Groot AJ, Habets R, Prickaerts J, Chalmers AJ, Eekers DBP, Theys J, Short SC, Verhaegen F, Vooijs M. NOTCH blockade combined with radiation therapy and temozolomide prolongs survival of orthotopic glioblastoma. Oncotarget 2016; 7: 41251-41264. doi: 10.18632/oncotarget.9275.

18. Zhang Z, Wodzak M, Belzile O, Zhou H, Sishc B, Yan H, Stojadinovic S, Mason RP, Brekken RA, Chopra R, Story MD, Timmerman R, Saha D. Effective Rat Lung Tumor Model for Stereotactic Body Radiation Therapy. Radiat. Res. 2016; 185: 616-622. doi: 10.1667/RR14382.1.

19. Panth KM, Leijenaar RTH, Carvalho S, Lieuwes NG, Yaromina A, Dubois L, Lambin P. Is there a causal relationship between genetic changes and radiomics-based image features? An in vivo preclinical experiment with doxycycline inducible GADD34 tumor cells. Radiother. Oncol. 2015; 116: 462-466. doi: 10.1016/j.radonc.2015.06.013.

20. Yahyanejad S, van Hoof SJ, Theys J, Barbeau LMO, Granton PV, Paesmans K, Verhaegen F, Vooijs M. An image guided small animal radiation therapy platform (SmART) to monitor glioblastoma progression and therapy response. Radiother. Oncol. J. Eur. Soc. Ther. Radiol. Oncol. 2015; 116: 467-472. doi: 10.1016/j.radonc.2015.06.020.

21. Cramer CK, Yoon SW, Reinsvold M, Joo KM, Norris H, Hood RC, Adamson JD, Klein RC, Kirsch DG, Oldham M. Treatment Planning and Delivery of Whole Brain Irradiation with Hippocampal Avoidance in Rats. PloS One 2015; 10: e0143208. doi: 10.1371/journal.pone.0143208.

22. Maastricht Health Campus. Brightlands: research and business in materials and health Available from: https://www.brightlands.com/brightlands-maastricht-health-campus.

23. MURIPLAN. Xstrahl Medical \& Life Sciences Available from: https://xstrahl.com/life-sciencesystems/muriplan/.

24. Fedorov A, Beichel R, Kalpathy-Cramer J, Finet J, Fillion-Robin J-C, Pujol S, Bauer C, Jennings D, Fennessy F, Sonka M, Buatti J, Aylward S, Miller JV, Pieper S, Kikinis R. 3D Slicer as an Image Computing Platform for the Quantitative Imaging Network. Magn. Reson. Imaging 2012; 30: 1323-1341. doi: 10.1016/j.mri.2012.05.001.

25. RaySearch releases micro-RayStation for pre-clinical research - RaySearch Laboratories. Available from: https://www.raysearchlabs.com/press/?year=2017\&cisionid=2773432.

26. Radiochromic film dosimetry - Radiochromic.com. Available from: https://radiochromic.com/.

27. Oncoradiomics - A I - Radiomics Software - Clinical \& Research. Oncoradiomics - A I - Radiomics Software - Clinical \&amp; Research Available from: https://www.oncoradiomics.com.

28. FALCON. Available from: https://www.estro.org/school/articles/e-learning/falcon/falcon. 
Acknowledgments 

Dank beste lezer, dat je bent toegekomen aan bijna het einde van mijn proefschrift. Misschien is dit het eerste dat je leest, wat dan ook, dank voor nemen van de tijd want voor mij vormt dit fysieke exemplaar van mijn proefschrift het eindpunt van een aantal waardevolle jaren. Een mooie tijd waarop ik graag wil terugblikken en een aantal personen bedanken die daaraan hebben bijgedragen.

Mijn promotor Frank Verhaegen, zoals je zelf zegt stel je als supervisor altijd hoge eisen aan je studenten, of het nu bachelor-, master-, of PhD-studenten zijn. Als voormalig master- en PhD-student onder je hoede kan ik dat beamen. In je rol als wetenschappelijke mentor wil ik in het bijzonder je harde-werken mentaliteit, toegankelijkheid, ongeëvenaarde snelle responsiviteit en hoge productiviteit benoemen, en dat alles in de variëteit van de onderzoekslijnen die je leidt. Je bent vooral zeer betrokken en neemt ruim de tijd voor al je studenten, ook al heb je zelf een gigantisch pakket werk liggen met daarbij horende deadlines. Dat is zeker iets dat erg gewaardeerd wordt. Ik wil hierin ook graag je vrouw Brigitte betrekken. Onze samenwerking ging verder bij het starten van SmART Scientific Solutions B.V.; we werken aan nog vele jaren succesvol ondernemen, in combinatie met onderzoek!

Second, the Canadian who was there during my first day at Maastro to introduce project topics, who helped me publish my first paper, who helped me graduate from university, for whom I was there during his defense as paranymph; Patrick. Patrick, thank you for all the guidance and patience during these years. You found a lovely wife here as well, went back to Canada and back to the Dutch, who knows what the future holds more, I can only hope for the best. Enjoy your new family life with Hudson, we're still connected through our company so to many more years of success!

The assessment committee, chairman prof. dr. De Ruysscher, associate prof. dr. Dubois, prof. dr. Georg and associate prof. dr. Vanhove, thank you for freeing up time in your busy schedules to appraise my thesis.

Ralph, we begonnen jaren geleden ongeveer tegelijk bij Maastro en hebben heel wat bakkies weggetikt op de tweede en derde verdieping, met een goed diepgaander gesprek maar zeker vaker louter oppervlakkig gezwets. Het startup leven leek jou ook wel iets en we zien elkaar daardoor gelukkig nog af en toe op de campus, waar we nu vooral onszelf managen in de C-suite. Dank dat je ook tijdens mijn verdediging achter me staat als paranimf! Ik wens je het allerbeste in de academische en startup wereld, tot snel!

Shane, we were good neighbors for many years in the office, during which I tried to keep your stuff at your side, with varying success. Your presence in the office assures 
a never ending stream of conversational topics. Be sure to write a novel some day, I want to buy the first copy! Thanks also for all the late-hour chats, I wish you all the best, wherever you will end up. Brent, Joana, it was great to help you getting your master's degree, it were good times and I learned a lot as well. Brent je eigen PhD gaat sneller dan je misschien had verwacht, succes met het laatste gedeelte en wat daarna ook mag komen. Ik wens je, misschien vooral, veel feest, met je part time carrièreswitch, the best is yet to come! Succes als paranimf (= Joana, cheerful at all times, your likeable character made the projects we did all the better, thanks a lot. Hopefully you'll free up some time to show me around in Lisbon someday, good luck with your job in Portugal! Lucas, altijd in voor voor een praatje, of een heel gesprek, dank voor de dicomadviezen, bij ICT kwamen we elkaar al snel weer tegen met beide andere functietitels, we zullen elkaar zeker weer snel zien. Daniela, you are a very kind and smart person, thank you for the nice collaborations we had, see you in Maastricht.

People from Precision X-Ray, William McLaughlin, Paul De Jean, Kiran Beera. Thanks for our nice and ongoing collaboration, for the help with the XRAD when we were pushing it to its limits once again. Paul, never a boring conversation with you, I hope someday your chronic jet lag will be over, I wish you all the best with the new company. Hopefully we'll be able to chat about it someday whilst enjoying a good Belgian beer, we'll bring a film scanner for extra joy! To Robert Weersink and James Stewart, thank you for the hospitality when I visited Toronto, I had a great and valuable time, good luck with your further careers in radiotherapy.

Alle SmART-gebruikers die ik door de jaren heen heb mogen ondersteunen, ik heb wat kilometers afgelegd tussen Maastro en RNL/Maastro Lab, en wat uren doorgebracht in die prachtige ruimte bij de SmART. Het was leuk en interessant om wat meer van het lab mee te krijgen. Ludwig en Natasja, jullie kunnen mijn nummer onder speeddial houden onder "SmART remote support", we zullen dan samen proberen om dat grijze stalen monster weer onder controle te krijgen. Martine en Sandra van RNL, sorry voor de 'SmART' smeervlekken in de labjassen, dank voor de ondersteuning. Judith, het besluit om de analyse volledig te scripten en automatiseren was geweldig en vreselijk, maar uiteindelijk zeer waardevol! Mooi om te zien hoe we met jullie de beeldvorming op weer een andere manier kunnen inzetten en dat het project nu door anderen wordt voortgezet. Dank voor de fijne samenwerking en hopelijk zien we elkaar nog eens. Venus, you've been through tough times but you did it, your PhD is in the pocket. I hope you'll find a great new research group and project; may I recommend something that does not involve (3D) BLI? Thank you for your kindness! Sanaz, 
the animal work certainly demanded a lot of your energy and time, lab work can be very challenging, something which 'we' from the dry lab can underestimate. You are probably the friendliest person l've met, it is great that we got to do some research together. See you around! Linda, ik heb mijn best gedaan om met mijn technische achtergrond je studie zo goed mogelijk te presenteren in Lissabon; helaas gingen de vragen niet over de deformatie-analyse waar ik wel alles vanaf wist... Succes met je verdere carrière en gezinsleven buiten onderzoek! Also Rianne, Ala, Kranthi, it was great working with you on the studies involving the SmART.

Professor Dick den Hertog, Marleen Balvert, en Marjolein Kroon van de afdeling Econometrics and Operations Research van Tilburg University, en Aswin Hoffmann die deze samenwerking tot stand bracht. We hadden wat opstarttijd nodig om elkaars taal te leren spreken, maar uiteindelijk is er een mooie synergie onstaan door het combineren van onze vakgebieden. Dank voor de leuke en leerzame samenwerking!

There are many more colleagues l've met throughout the years, without pursuing an exhaustive complete list, and in no particular order: Janita, Karen, Isabel, Ruben, Seán, Timo, Evelyn, Adriana, Aniek, Sara, Ana, Cecile, Gabriel, José, Jurgen, Lotte, Mark, Murillo, Relinde, Skadi, Celine, Guillaume, Hoda, Davide, Raghu, Turkey, Jean, Johan, Sebastian, Mariana, Scott, Yvonka, Jolanda. It was great to be colleagues, to share lunches, coffee breaks, foreign sweet selections, walking lunches, shoot and edit PhD movies, join conferences, have drinks, celebrate carnaval. Thanks for all the good times, undoubtedley you'll all have a fantastic further career, in or outside radiotherapy!

Er is zeker ook een leven buiten werk, met natuurlijk voor mij grote bijdragen daaraan door mijn vrienden van Nummerke Drie, Bag on Wheels, en $\approx$ VVB. Jullie hebben zonder twijfel een grote bijdrage gehad om er een gezellige tijd van te maken. Vaak hebben we geen idee wat de ander eigenlijk doet naast zijn vrije tijd, en dat is misschien wel prima, zodat we echt af kunnen schakelen van werk. Ik prijs me ook gelukkig met mijn twee fantastische ouders die erg trots op me zijn, met mijn twee lieve grote broers Joost en Erik, en lieve schoonzussen Simone en Rian, en natuurlijk (aankomende) neefje(s) en nichtje(s)! Dank voor het tonen van interesse en ondersteuning!

Gaby, jij geeft me onvoorwaardelijke steun, al vele jaren, je geeft me de tijd als ik die nodig heb voor andere dingen, ook ten koste van jezelf. Je hebt misschien geen idee hoe zeer dat bijdraagt aan het verwezenlijken van bijvoorbeeld dit proefschrift. We 
kennen elkaar inmiddels zo goed, ik denk niet dat iets of iemand dat ooit nog kan veranderen. Dankjewel $\triangleright$ ! Lest best, als laatste iemand die dit de komende jaren nog niet zal lezen en begrijpen: Jasmijn. Net voor het afronden van het laatste hoofdstuk van mijn proefschrift gaf jij me al de allermooiste titel: papa! Je hebt onze levens nu al voor altijd veel mooier gemaakt, ik kan niet wachten op wat we allemaal nog gaan meemaken samen!

Stefan van Hoof

Maastricht

July 3, 2019 
Curriculum Vitae 



\section{Curriculum Vitae}

Stefan van Hoof was born on the $16^{\text {th }}$ of February 1987 in Geldrop, the Netherlands. After finishing his secondary education at Carolus Borromeus College in Helmond in 2005, he enrolled in the bachelor program Biomedical Engineering at Eindhoven University of Technology. He continued with the master program Medical Engineering at the same university in collaboration with Maastricht University. During this master he completed an internship at the Howard Florey Institute (Melbourne, Australia) working on dynamic contrast enhancement using magnetic resonance imaging for multiple sclerosis.

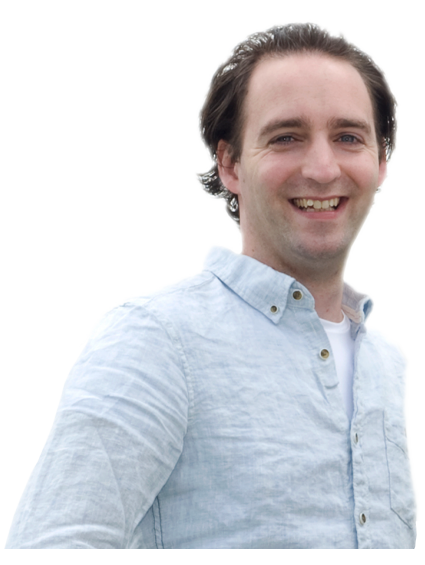
He completed that master at Maastro Clinic, working on triple channel radiochromic film dosimetry for preclinical radiotherapy in 2012. After graduating, he continued his scientific career at Maastro Clinic to work on radiation planning for image guided preclinical radiotherapy, and soon converted his position into a PhD track at Maastricht University under the supervision of professor Frank Verhaegen. This work led to comprehensive tools for radiation planning that were, and are still used at many research institutes worldwide. In 2015, he co-founded the company SmART Scientific Solutions B.V. at Brightlands Maastricht Health Campus during his PhD program, to further professionalize these activities. Currently, Stefan is working there to lead software development to further improve preclinical radiation planning, to increase the customer base, and to expand the product portfolio into other markets.

\section{Publications}

- van Hoof SJ, Verde JB, Verhaegen F. Dose painting by dynamic irradiation delivery on an image-guided small animal radiotherapy platform. Br J Radiol 2019; 92: 20180744.

- Sosa Iglesias V, van Hoof SJ, Vaniqui A, Schyns LE, Lieuwes N, Yaromina A, Spiegelberg L, Groot AJ, Verhaegen F, Theys J, Dubois L, Vooijs M. An orthotopic non-small cell lung cancer model for image-guided small animal radiotherapy platforms. Br J Radiol 2018; 20180476. 
- Persoon L, van Hoof SJ, van der Kruijssen F, Granton P, Sanchez Rivero A, Beunk H, Dubois L, Doosje J-W, Verhaegen F. A novel data management platform to improve image-guided precision preclinical biological research. $\mathrm{Br} J$ Radiol 2018; 91 : 20180455.

- van der Heyden B, Podesta M, Eekers DB, Vaniqui A, Almeida IP, Schyns LE, van Hoof SJ, Verhaegen F. Automatic multiatlas based organ at risk segmentation in mice. Br J Radiol 2018; 91: 20180364.

- Vaniqui A, van der Heyden B, Almeida IP, Schyns LE, van Hoof SJ, Verhaegen $F$. On the determination of planning target margins due to motion for mice lung tumours using a four-dimensional MOBY phantom. Br J Radiol 2018; 91: 20180445.

- Ceelen JJM, Schols AMWJ, Kneppers AEM, Rosenbrand RPHA, Drożdż MM, van Hoof SJ, de Theije CC, Kelders MCJM, Verhaegen F, Langen RCJ. Altered protein turnover signaling and myogenesis during impaired recovery of inflammation-induced muscle atrophy in emphysematous mice. Sci Rep 2018; 8: 10761.

- Vaniqui A, Schyns LEJR, Almeida IP, van der Heyden B, van Hoof SJ, Verhaegen $F$. The impact of dual energy CT imaging on dose calculations for pre-clinical studies. Radiat Oncol 2017; 12: 181.

- van der Heyden B, van Hoof SJ, Schyns LEJR, Verhaegen F. The influence of respiratory motion on dose delivery in a mouse lung tumour irradiation using the 4D MOBY phantom. Br J Radiol 2017; 90: 20160419.

- Schyns LEJR, Almeida IP, van Hoof SJ, Descamps B, Vanhove C, Landry G, Granton PV, Verhaegen F. Optimizing dual energy cone beam CT protocols for preclinical imaging and radiation research. Br J Radiol 2017; 90: 20160480.

- De Ruysscher D, Granton PV, Lieuwes NG, van Hoof SJ, Wollin L, Weynand B, Dingemans A-M, Verhaegen F, Dubois L. Nintedanib reduces radiation-induced microscopic lung fibrosis but this cannot be monitored by CT imaging: A preclinical study with a high precision image-guided irradiator. Radiother Oncol 2017; 124: 482-487.

- Ceelen JJM, Schols AMWJ, van Hoof SJ, de Theije CC, Verhaegen F, Langen RCJ. Differential regulation of muscle protein turnover in response to emphysema and acute pulmonary inflammation. Respir Res 2017; 18: 75. 
- Yahyanejad S, King H, Iglesias VS, Granton PV, Barbeau LMO, van Hoof SJ, Groot AJ, Habets R, Prickaerts J, Chalmers AJ, Eekers DBP, Theys J, Short SC, Verhaegen F, Vooijs M. NOTCH blockade combined with radiation therapy and temozolomide prolongs survival of orthotopic glioblastoma. Oncotarget 2016; 7 : 41251-41264.

- Yahyanejad $\mathrm{S}^{*}$, van Hoof $\mathbf{S J}^{\star}$, Theys $\mathrm{J}^{\star}$, Barbeau LMO, Granton PV, Paesmans K, Verhaegen F, Vooijs M. An image guided small animal radiation therapy platform (SmART) to monitor glioblastoma progression and therapy response. Radiother Oncol 2015; 116: 467-472.

- Balvert $\mathrm{M}^{\star}$, van Hoof $\mathbf{S J}^{\star}$, Granton PV, Trani D, den Hertog D, Hoffmann AL, Verhaegen F. A framework for inverse planning of beam-on times for 3D small animal radiotherapy using interactive multi-objective optimisation. Phys Med Biol 2015; 60: 5681-5698.

- Verhaegen F, van Hoof SJ, Granton PV, Trani D. A review of treatment planning for precision image-guided photon beam pre-clinical animal radiation studies. $Z$ Med Phys 2014; 24: 323-334.

- Granton PV, Dubois L, van Elmpt W, van Hoof SJ, Lieuwes NG, De Ruysscher $D$, Verhaegen F. A longitudinal evaluation of partial lung irradiation in mice by using a dedicated image-guided small animal irradiator. Int J Radiat Oncol Biol Phys 2014; 90: 696-704.

- Lindsay PE, Granton PV, Gasparini A, Jelveh S, Clarkson R, van Hoof SJ, Hermans J, Kaas J, Wittkamper F, Sonke J-J, Verhaegen F, Jaffray DA. Multiinstitutional dosimetric and geometric commissioning of image-guided small animal irradiators. Med Phys 2014; 41: 031714.

- van Hoof SJ, Granton PV, Verhaegen F. Development and validation of a treatment planning system for small animal radiotherapy: SmART-Plan. Radiother Oncol 2013; 109: 361-366.

- van Hoof SJ, Granton PV, Landry G, Podesta M, Verhaegen F. Evaluation of a novel triple-channel radiochromic film analysis procedure using EBT2. Phys Med Biol 2012; 57: 4353-4368.

\footnotetext{
* indicates equal contribution
} 
